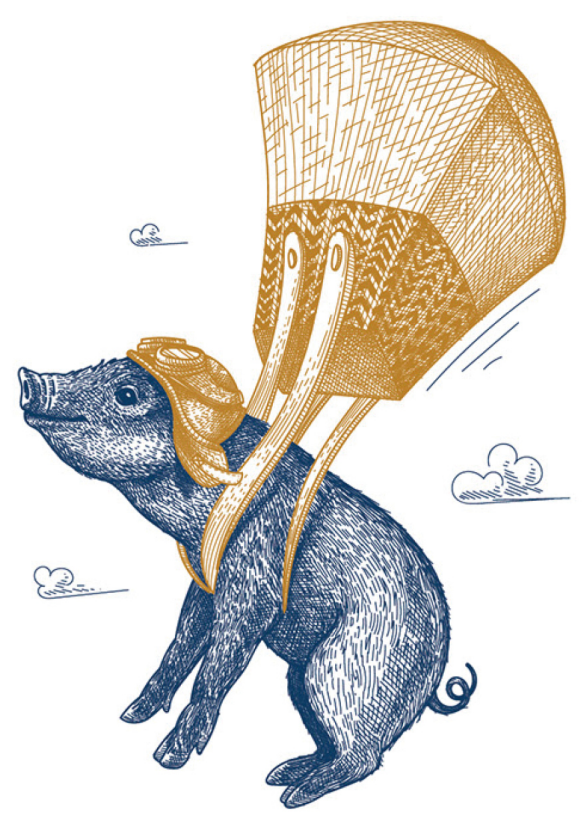

\title{
Eifects of early life and current environmental enrichment on behaviour, affective state and immunity in pigs
}

L u Luo

罗露

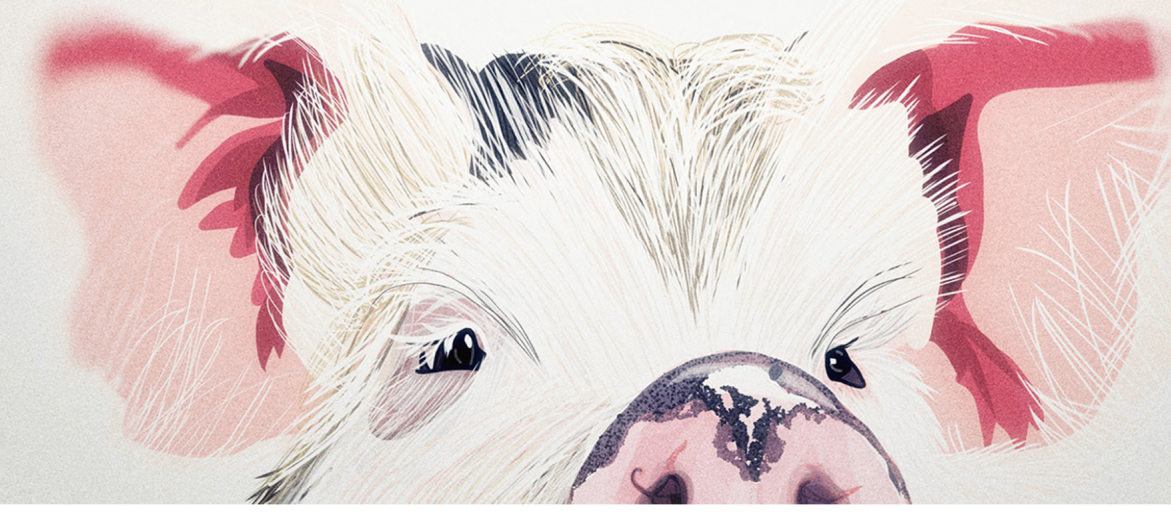




\section{Invitation}

You are cordially invited to attend the public defence of my PhD thesis entitled:

Effects of early life and current environmental enrichment on behaviour, affective state and immunity in pigs

On Friday the $24^{\text {th }}$ of April 2020 at 11.00 a.m.

Aula Wageningen University Generaal Foulkesweg 1 6703 BG Wageningen

Lu Luo

lu.luo@wur.nl

Paranymphs

Junnan Ma

junnan.ma@wur.nl

Francesca Marcato francesca.marcato@wur.nl 


\section{Propositions:}

1. The impact of the current environment on behaviour and immunity of pigs is dependent on their early life environment. (this thesis)

2. Pigs kept in barren conditions in early life are more sensitive to reward loss than pigs reared in enriched environment.

(this thesis)

3. Natural antibody levels are potential phenotypical indicators of resilience.

4. Data analysis is a way to approach facts.

5. Consumption of animal products will decrease with increasing awareness of mental capabilities of animals.

6. It is easier to go from frugality to extravagance than from extravagance to frugality.

Propositions belonging to the thesis, entitled:

"Effects of early life and current environmental enrichment on behaviour, affective state and immunity in pigs"

Lu Luo

Wageningen, 24 April 2020 
Effects of early life and current environmental enrichment on behaviour, affective state and immunity in pigs

Lu Luo 


\section{Thesis committee}

\section{Thesis committee}

\section{Promotor}

Prof. Dr B. Kemp

Professor of Adaptation Physiology

Wageningen University \& Research

\section{Co-promotors}

Dr J.E. Bolhuis

Associate professor, Adaptation Physiology Group

Wageningen University \& Research

Dr H.K. Parmentier

Associate professor, Adaptation Physiology Group

Wageningen University \& Research

\section{Other members:}

Prof. Dr M. Naguib - Wageningen University \& Research

Prof. Dr A.B. Lawrence - Scotland's Rural College (SRUC), Midlothian, United Kingdom

Dr. E. Merlot - French National Institute for Agriculture, Food and Environment, INRAE, Saint-Gilles, France

Dr. R.E. Nordquist - Utrecht University

This research was conducted under the auspices of the Graduate School Wageningen Institute of Animal Sciences (WIAS) 


\title{
Effects of early life and current environmental enrichment on behaviour, affective state and immunity in pigs
}

\author{
Lu Luo
}

Thesis

submitted in fulfilment of the requirements for the degree of doctor

at Wageningen University

by the authority of the Rector Magnificus,

Prof. Dr A.P.J. Mol,

in the presence of the

Thesis Committee appointed by the Academic Board

to be defended in public

on Friday 24 April 2020

at 11 a.m. in the Aula 
Lu Luo

Effects of early life and current environmental enrichment on behaviour, affective state and immunity in pigs, 332 pages

PhD thesis, Wageningen University, Wageningen, the Netherlands (2020)

With references, with summary in English

ISBN 978-94-6395-329-0

DOI: https://doi.org/10.18174/516289 


\section{Contents}

\section{Chapter 1}

General introduction

Chapter 2

Effects of environmental enrichment and regrouping on natural autoantibodies-binding danger and neural antigens in healthy pigs with different individual characteristics

Chapter 3

Effect of enriched housing on levels of natural (auto-) antibodies in pigs coinfected with porcine reproductive and respiratory syndrome virus (PRRSV) and Actinobacillus pleuropneumoniae

Chapter 4

Effects of early and current environmental enrichment on behaviour and growth in pigs

\section{Chapter 5}

Effects of early and later life environmental enrichment and personality on attention bias in pigs (Sus scrofa domesticus)

Chapter 6

Effects of early life and current housing on sensitivity to reward loss in a successive negative contrast test in pigs

Chapter 7

Early and later life environmental enrichment affect specific antibody responses and blood leukocyte subpopulations in pigs

Chapter 8

General discussion 
Acknowledgements

About the author

353

Education statement 355 


\section{Chapter 1}

General Introduction 



\subsection{Introduction}

Welfare and health issues in farm animals, including pigs, have become increasingly important to the public as well as in science. On commercial farms, most pigs are housed in rather barren, stimulus-poor conditions, that poorly meet their behavioural and psychological (emotional) needs and hypo-stimulate their senses and cognitive abilities. Pigs in these stimuluspoor environments often show behavioural, physiological, and pathological signs of poor welfare and stress (Bolhuis et al. 2013; Carreras et al. 2016; Munsterhjelm et al. 2010; Yang et al. 2018), which could potentially also impact their immune competence and health. Apart from the current environment, experiences in early life can have long-term effects (Archard et al. 2012; Bolhuis et al. 2006; Chaby et al. 2013; Chiang et al. 2015; Lukkes et al. 2009; Munsterhjelm et al. 2009; Munsterhjelm et al. 2010; Schmeer et al. 2019; Sheriff et al. 2009), and, therefore the effects of housing may also depend on early life housing history. This thesis will focus on the effects of early life and current environmental enrichment as opposed to barren housing on behaviour, affective states, and immunity in pigs.

In this chapter, first a background of the welfare problems of pigs kept in stimulus-poor environments in commercial pig husbandry is given. Thereafter, environmental enrichment is introduced, as well as its effect on different aspects of animal welfare. Subsequently, the potential effects of experience with enrichment materials in early life and of a (mis)match with current housing on behaviour, affective state and health are described. The question is raised whether and how the interplay between early and current 
enrichment may impact pig welfare, including health, and lastly, the aim and outlines of this thesis are presented.

\subsection{Pig husbandry and welfare problems}

Since the 1960s, pig production has dramatically changed from small familyrun farms to large production units in industrialised countries (Pedersen 2018). These changes have shifted the extensive housing systems with a high labour input to economically efficient units with low labour input, low space allowance, and use of slatted or partly slatted floor systems with little or no bedding materials (van de Weerd and Day 2009). Intensive indoor systems have a number of advantages such as protection of pigs against predators, climate control, better controlled hygiene, and ease of cleaning and management (Bammert et al. 1993). However, several features of intensive indoor systems have come at a cost for pig welfare. The limited living space and absence of substrates or materials to root in, give rise to major welfare problems in pigs in intensive indoor systems. Pigs are intelligent and social animals with a wide range of inquisitive behaviours. In a natural environment, pigs spend a large part of their active time on searching for food and collecting information by exploring their surroundings within a large home range area (see Studnitz et al. 2007 for review). The execution of these behaviours is hampered in intensive systems (reviewed in Barnett et al. 2001). When pigs do not have opportunities to show their explorative behaviour, they direct more activities to their physical and social surroundings. For instance, they root the bare floor and chew air (Petersen et al. 1995), but also show oral manipulation of their pen mates' ears, tails and other body parts (Kallio et al. 2018; Moinard et al. 2003; Petersen et al. 1995; Scollo et al. 2016; 
Taylor et al. 2012), leading to stress and poor performance (Dudink et al. 2006; Oostindjer et al. 2011; Widowski et al. 2008). Once learned, these maladaptive behaviours, such as tail biting, can persist within groups of pigs, leading to increased risks of infection through bite injuries (e.g. Fraser et al. 1991; Guy et al. 2002; Scott et al. 2006) and production losses (e.g. Beattie et al. 2000b; Beattie et al. 1996; Jordan et al. 2008), thereto reduced welfare (Camerlink et al. 2012; Edwards 2006; Taylor et al. 2010). Tail docking is an efficient way to reduce tail biting in current intensive housing systems for pigs, but it does not completely eliminate the problem when unfavourable conditions persist. It is painful and can cause long-term welfare problems (European Food Safety Authority, 2007). Tail docking is not allowed to be performed routinely in the EU, yet it is still widely applied in many EU countries. Societal pressure is increasing to ban this practice and, therefore, alternative methods to prevent pigs from developing damaging behaviours are urgently needed.

Over recent decades, due to the welfare problems caused by barren housing, alternative housing systems, such as outdoor housing and organic farming, and application of environmental enrichment on conventional farms, have gained interest. Environmental enrichment is an important requirement for the welfare of pigs as it allows them to perform more of their species-specific behavioural repertoire (reviewed in Studnitz et al. 2007; van de Weerd and Day 2009), and environmental enrichment with rooting substrates seems to be a promising way to reduce the tail biting and other damaging behaviours (Beattie et al. 1995; Taylor et al. 2010; Zonderland et al. 2008). 


\subsection{Environmental enrichment}

Environmental enrichment can be defined as "an increase of the biological relevance of captive environments by appropriate modifications resulting in an improvement of the biological functioning of captive animals" (Newberry 1995). In laboratory research, environmental enrichment usually refers to a housing condition combining complex inanimate (extra space, novel materials, and objects) and social (pen mates) stimuli that are supposed to enhance sensory, cognitive, motor and social skills compared with standard housing conditions consisting of rather bare cages (reviewed in Jirkof 2015). The scientific study of environmental enrichment began to gain interest in the 1960s. The early studies focused on the effects of enrichment on learning ability and brain plasticity (reviewed in Renner and Rosenzweig 1987), and in the 1970s, zoos began to employ enrichment to enhance the expression of natural behaviour and to reduce the incidence of behavioural problems in captive animals (Shepherdson 1998). Then it shifted towards studies on improving animal welfare in laboratories and farms as the science of enrichment matured (Chamove 1989; Markowitz and Gavazzi 1995; Mench 1994), and enrichment was mainly compared to standard housing conditions (Ball et al. 2019). Application of environmental enrichment generally aims to improve the animals' quality of life by providing them with a variety of cognitive, multisensory stimuli, and by eliciting natural exploratory behaviour (Baroncelli et al. 2010). Studies in laboratory animals have shown that environmental enrichment can reduce reactivity to stress and anxiety (Varman et al. 2012; Veena et al. 2009), and increase cognitive functions (Arai and Feig 2011), shown by enhanced learning and memory mechanisms (Arai and Feig 2011; Van Praag et al. 2000). 
Behavioural problems in intensive pig husbandry systems have led to a large amount of research on the potential for environmental enrichment to improve welfare. There are different types of environmental enrichment for pigs, such as more space, provision of rooting materials, offering 'toys', i.e. objects for oral manipulation (van de Weerd and Ison 2019) and cognitive stimulation (Franks 2018; Rauterberg et al. 2013; Sonoda et al. 2013; Zebunke et al. 2013).

Provision of substrates or materials for exploring is a major method to combat welfare problems in pigs, as these can satisfy their behaviour need to chew and root. These substrates or materials need to be chewable, deformable, destructible and ingestible to be effective (Van de Weerd et al. 2003). Since January 2003, the provision of appropriate environmental enrichment to pigs of all ages has been mandatory across the European Union (EU) (Directives 2001/88/EC and 2001/93/EC). The Directives state that: "To enable proper investigation and manipulation activities, all pigs must have permanent access to a sufficient quantity of material such as straw, hay, wood, sawdust, mushroom compost, peat or a mixture of such which does not adversely affect the health of the animals". Yet, it should be noted that in several countries within (and outside) the EU farmers are reluctant to use such materials in intensive systems, because of labour, costs, concerns about hygiene, and incompatibility with the (partly) slatted floors and manure handling systems, and they rather provide objects that are less satisfying for pigs, such as chains with a ball, or plastic pipes (Bracke and Koene 2019). Environmental enrichment could also involve extra space, and it has been found that more space allowance can reduce skin lesions, reflecting lower levels of aggression, and increase growth in pigs (Randolph 
et al. 1981; Turner et al. 2000). However, Beattie et al. (1996) demonstrated that substrate-enrichment played a greater role in determining pigs' behaviour than space allowance, and this was particularly true for the occurrence of damaging behaviours. Studies on enrichment in pigs therefore mostly address the provision of substrates for exploration, either or not in combination with extra space and toys, and will the focus of this chapter and studies within my PhD project.

\subsubsection{Environmental enrichment and animal welfare}

Fraser (2008a) reported what is now widely accepted as the three constituents of animal welfare: (1) animals should have the ability to live reasonably natural lives consistent with their evolutionary history, which normally satisfy the motivation to express their natural behaviour; (2) animals should experience minimal negative psychological states and the presence of at least some positive psychological states, so this constituent is about the "feelings" of the animals, further referred to as affective state; and (3) animals should exhibit good physical health and biological functioning. These three constituents overlap to some extent as, for instance, it is believed that allowing animals to live a more natural life would make them more happy and more healthy (Fraser 2008b). Below, I am going to introduce the (putative) effects of environmental enrichment in the form of rooting substrates either or not in combination with extra space, on the three constituents of welfare.

\subsubsection{Natural behaviour}

Environmental enrichment can enhance the welfare of animals by allowing them to perform more of their species-specific behaviour and accommodate 
a larger range of behavioural choices (reviewed in van de Weerd and Day 2009). Studies on enrichment for farm animals, including pigs, mostly focus on behavioural outcomes. Enrichment of the environment of pigs with rooting substrates, such as straw or peat, either or not in combination with extra space, generally reduces time spent on damaging behaviours directed at pen mates (i.e. tail biting and ear biting), while increasing the time spent on activity, exploratory and play behaviours (e.g. Beattie et al. 2000b; Bolhuis et al. 2005; Mkwanazi et al. 2019; Oliveira et al. 2016; Ursinus et al. 2014).

Apart from affecting their time budget, enrichment may also influence behaviour of pigs in cognitive tasks. For instance, pigs in enriched environments more rapidly learned an operant task and spatial learning task than their counterparts from barren environments (Sneddon et al. 2000). Environmental enrichment also improved working memory of pigs in a spatial discrimination task (Bolhuis et al. 2013; Grimberg-Henrici et al. 2016), and enhanced long-term spatial memory (de Jong et al. 2000), although effects on reference memory were not consistent (no effect: Bolhuis et al. 2013; improvement: Grimberg-Henrici et al. 2016). Others (de Jong et al. 2000; Jansen et al. 2009) did, however, not find beneficial effects of enrichment on acquisition of a spatial task. It should be noted that in most of these studies enrichment consisted of rooting substrates and extra space, which may be more effective in stimulating (spatial) cognition than substrates alone.

The enhanced performance of enriched pigs as compared to their barren counterparts might result from improved early life development of cognitive abilities. Studies in rodents indicate that environmental enrichment affects 
brain development (Rosenzweig and Bennett 1996), which is reflected in improved spatial learning (e.g. Leggio et al. 2005; Schrijver et al. 2002). Moreover, also in pigs, (substrate) enrichment promotes play behaviour (e.g. Bolhuis et al. 2005), which may be important for cognitive development and behavioural flexibility (Held and Špinka 2011; Spinka et al. 2001). Additionally, the better performance of enriched as compared to barren housed pigs could also reflect chronic stress in the latter group, resulting from a lack of suitable rooting substrates and/or space in their home pen environment (Bolhuis et al. 2013). Stress may influence cognitive processes in different ways, with mild acute stress sometimes even improving learning and memory, whereas chronic stress mostly negatively interferes with cognitive processes (reviewed in Mendl 1999). It has been suggested that improved cognitive skills may benefit welfare by facilitating adaptation to husbandry challenges (Franks 2018), although it should be noted that clever animals also may be at greater risk for boredom if they are placed in cognitively less challenging environments (Nicol 1996).

To conclude, environmental enrichment, compared to barren housing conditions, improves the welfare of pigs by allowing them to express more natural behaviours. This conforms to the first constituent of the definition from Fraser (2008a). Enrichment also affects pigs' cognitive abilities, which may have equivocal consequences for welfare.

\subsubsection{Affective state}

Consistent with the second constituent of the animal welfare definition of Fraser (2008a), many modern definitions of welfare include a reference to the psychological state and feelings of animals, like whether an animal is 
'happy' or not (e.g. Boissy et al. 2007; Dawkins 1988; Webster 2008), here referred to as affective state. The impact of environmental enrichment on affective state has been less well studied than its impact on natural behaviour.

Affective state is a combination of background mood and current emotional response (Mendl et al. 2010), which can influence each other (Murphy et al. 2014). Emotion is defined as a "specific, intense, and short response to stimuli", and mood is defined as "longer, more ambiguous, and nonattributable affective feelings of longer intensity" (Murphy et al. 2014; Schnall 2010). Affective state, both emotion and mood, can be characterized, on a two-dimensional scale, by valence (positive/negative) and arousal (high/low) (Mendl et al. 2009; Paul et al. 2005). Valence is a subjective feeling of pleasantness or unpleasantness, and arousal is a subjective state of feeling activated or deactivated (Barrett 1998).

Given the absence of direct measures of affective state, indirect behavioural and physiological measures are used, as affective states are multifaceted and comprise behavioural and physiological changes, in addition to subjective experiences. Tests used for assessing affective state in enriched vs. barren housed pigs include those measuring fearfulness or anxiety, and judgement bias reflecting optimism and pessimism. Behavioural tests to measure the negative affective states of fear and anxiety often assess the response of animals to novelty, e.g. in human approach tests (e.g. Reimert et al. 2014a) open field tests (e.g. Romeo et al. 2003; Romeyer and Bouissou 1992), novel object tests (e.g. Dalmau et al. 2009; Richard et al. 2008; Romeyer and 
Bouissou 1992), or their combination (e.g. Reimert et al. 2014a; Van Reenen et al. 2009).

It is assumed that the response of animals in fear tests can also reflect the impact of long-term conditions (e.g. enrichment vs. barren housing) on their affective state. For instance, environmental enrichment reduced the fearfulness to novel objects placed in their home cage in parrots (Meehan and Mench 2002). In pigs, enrichment also reduced fearfulness in novelty tests. Straw-enriched pigs were faster to touch a human (e.g. Reimert et al. 2013) and novel object than barren housed pigs (Reimert et al. 2014a; Wemelsfelder et al. 2000), and behaved differently in a novel environment (Beattie et al. 2000a; Jansen et al. 2009). Some other studies also showed that salivary cortisol concentrations were influenced in the novelty tests, although the results were not consistent (de Jong et al. 1998; de Jong et al. 2000; Reimert et al. 2014a; van de Weerd and Day 2009).

Also, cognitive judgement bias tests have been used to assess the impact of environmental enrichment on affective state in pigs. These tests are based on the finding that affective states can alter cognitive processing and bias interpretation of ambiguous stimuli, with individuals in a more positive affective state interpreting such stimuli in a more optimistic manner than those in a negative affective state (reviewed in Mendl et al. 2009), i.e. 'the glass half full or half empty'. There is evidence to show that environmental enrichment influences judgement bias. For example, in rats (Brydges et al. 2011) and starlings (Bateson and Matheson 2007; Matheson et al. 2008), environmental enrichment induced a more positive affective state, indicated by a more optimistic cognitive response to ambiguous stimuli. Also pigs 
housed in an enriched environment showed more optimistic judgement bias in a go/no-go task, suggesting a more positive affective state as compared to barren housed pigs (e.g. Asher et al. 2016; Douglas et al. 2012). In contrast, Carreras et al. (2016) reported no difference between enriched and barren housed pigs in judgement bias despite negative effects of barren housing on physiological and behavioural welfare indicators. A disadvantage of judgement bias tests is that they require training of animals, which can be time-consuming, and not all animals can be trained successfully (Monk et al. 2018; Roelofs et al. 2016). Besides, the training may be actually enriching to animals, which could overrule or influence the effects of housing conditions on affective state. Other, additional measures of affective state are therefore needed to assess the impact of environmental enrichment.

Tests that, to my knowledge, have not been used in pigs yet, are an attention bias test and successive negative contrast test, which have been used to assess negative affective states in other species (e.g. rats: Burman et al. 2008; sheep: Lee et al. 2016 \& Monk et al. 2018; dog: Riemer et al. 2016) . Attention bias tests do not require training and are based on the finding in humans and other species suggesting that individuals with a negative affective state (e.g. anxiety or depression) seem to pay more attention to a potential threat (Bradley et al. 1997; Bradley et al. 1995; Lee et al. 2018; Monk et al. 2018). The principle of a successive negative contrast test is that it assesses the sensitivity to reward loss, i.e. 'disappointment', and the capacity to overcome such a loss (Burman et al. 2008; Chaby et al. 2013; Tucker and Luu 2007). A study in rats reported that rats from an aversive environment had more difficulty to recover from reward loss than enriched housed rats (Burman et al. 2008). 
In conclusion, studies on the impact of enrichment on affective states in pigs are rather scarce. Based on behaviour of pigs in fear tests and their responses in judgement bias tests, there is some evidence for enrichment to induce more positive affective states. However, more research, using different measures of affective state, is needed to confirm this.

\subsubsection{Biological functioning and physical health}

According to the third constituent of animal welfare, animals also should exhibit good physical health and biological functioning. This means that they should have satisfactory health, growth and normal functioning of physiological and behavioural systems (Fraser et al. 1997).

This constituent is often associated with the absence of a large or chronic physiological stress response, and with being able to 'cope' with the environment (reviewed in Duncan 2005). It has been suggested that barren housing conditions can induce chronic stress, and, in this way, negatively impact pigs' biological functioning and physical health. For example, pigs in barren environments had a higher baseline body temperature (de Jong et al. 2000) and more severe gastric lesions (Bolhuis et al. 2006) than pigs in enriched environments, which might (partly) be attributed to chronic stress (de Jong et al. 2000). In several studies, pigs kept without substrates showed changes in hypothalamic-pituitary-adrenal (HPA) axis functioning, such as increased basal levels of cortisol (Klont et al. 2001; Reimert et al. 2014a; van de Weerd and Day 2009), but see de Jong et al. (1998) who found lower basal levels in barren housed pigs. In addition, in other studies barren housing led to a blunted circadian rhythm in cortisol (de Jong et al. 2000; Munsterhjelm et al. 2010) and heavier adrenals at slaughter (Beattie et al. 2000a). It is 
known that the HPA axis can become accustomed to stress stimuli thus affecting cortisol levels, and it seems that barren housing induces changes in this axis which may be reflective of chronic stress (Becker et al. 1985; De Jonge et al. 1996; Janssens et al. 1994). In addition, several (e.g. Beattie et al. 2000b; Oostindjer et al. 2010), but not all (e.g. Caldara et al. 2012; Peeters et al. 2006) studies report that enriching the environment may improve the growth rates of pigs, which could be related to reduced chronic stress levels (reviewed in Mkwanazi et al. 2019).

Less is known about the impact of environmental enrichment on general health and immune competence in pigs. It is often assumed that enrichment with substrates, like straw, has a negative impact on hygiene and air quality and can induce the carry-over of diseases. Indeed, use of straw has been suggested as a risk factor for a number of infections (reviewed in Tuyttens 2005). On the other hand, when used as bedding material, straw reduces foot and leg problems (Gentry et al. 2002; Lyons et al. 1995), including the occurrence of leg injuries that may lead to entrance of pathogens.

Apart from influencing the microbes and nutrients pigs are exposed to via contact with and intake of the substrates provided, thereby potentially influencing the immune system and gut microbiota, environmental enrichment may also indirectly affect immune competence and health via its (putative) effect on stress and mood. The fields of neuroimmunology and psychoneuroimmunology have shown that the central nervous system and the immune system are intimately linked and do not function as independent systems (e.g. Dantzer 2004; Felten and Olschowka 1987; Jiang et al. 1998). The communication between brain and immune system is bidirectional. The 
nervous system can communicate with the immune system, for instance via the HPA axis (Capuron and Miller 2011) and the sympathetic nervous system (Sternberg 2006; Wohleb et al. 2015). The immune system, in turn, influences the central nervous system, primarily through cytokines derived from lymphocytes and other leukocytes (Banks and Erickson 2010; Capuron and Miller 2011). It has been shown that stress can disrupt key functional interactions between the immune system and brain, ultimately affecting mood and behaviour (reviewed in Anisman and Merali 2003; Wohleb et al. 2015). For instance, activation of microglia, the resident innate macrophages of the brain, has been implicated as a key regulator of mood and behaviour in the context of prolonged exposure to stress (reviewed in Schlegelmilch et al. 2011; Wohleb et al. 2015). In addition, pro-inflammatory cytokines have been suggested to contribute to mood disorders such as depression (reviewed in Forsythe et al. 2014; Wohleb et al. 2015). Inversely, chronic stress in childhood heightened inflammation in later life (Kiecolt-Glaser et al. 2011), and individuals with a more negative affective state poorly mount immune responses and may be at higher risk for illness than those with a positive affective state (reviewed in Barak 2006). Thus, the brain-to-immune communication allows behavioural and environmental variables ultimately to impinge upon immune function (reviewed in Barak 2006; Kusnecov and Rabin 1994; Mason et al. 2012), and vice versa (reviewed in Dantzer 2017; Wohleb et al. 2015).

There are some indications that environmental enrichment, as opposed to barren housing, affects immune (re)activity in pigs. Although de Groot et al. (2000) did not find much differences in leukocyte and lymphocyte distributions, nor in the proliferation response to Concanavaline $A$ and in vivo 
humoral and cellular immune response to keyhole limpet hemocyanin (KLH) between pigs in barren housing and those enriched with straw and extra space, others did find effects of environmental enrichment on the immune system of pigs. For example, Reimert et al. (2014b) found that enrichment with straw bedding decreased the neutrophil to lymphocyte ratio and haptoglobin levels, but increased natural antibody levels specific for KLH in pigs enriched with straw and sawdust bedding. More space and free access to a roofed outdoor run lowered the neutrophil numbers and blood lymphocyte proliferation and heightened the lymphocyte to neutrophil ratio, but this was only seen in one of two genotypes studied (Merlot et al. 2012). Moreover, van Dixhoorn et al. (2016) found that enriched housed pigs following an infection with porcine reproductive and respiratory virus (PRRSV) and Actinobacillus pleuropneumoniae (A. pleuropneumoniae) had a faster clearance of viral RNA in blood and less (severe) lung lesions than barren housed pigs, which suggests that environmental enrichment reduces susceptibility to this lung disease. Whether differences in antigenic exposure, stress or mood are involved in the impact of substrate enrichment on immunity and disease susceptibility in pigs remains unknown. Cognitive enrichment, however, also affected immune parameters in pigs (Ernst et al. 2006), suggesting that effects of enrichment on the immune system are not solely a result of differences in antigenic stimuli.

The study of Reimert et al. (2014b) provided first evidence that enrichment may influence circulating natural antibodies (NAb), as enrichment increased both IgM and IgG antibodies binding KLH in pigs, a glycoprotein they had never been exposed to. NAb, which are generally characterized by polyspecificity and low affinity, are important as first line of defence against 
microbial infections (Coutinho et al. 1995). They moreover prime the adaptive immune response (Lammers et al. 2004; Manson et al. 2005) and play a role in clearing apoptotic cells (Manson et al. 2005; Quartier et al. 2005). A large part of the NAb repertoire is directed to self-antigens, and these antibodies are referred to as natural autoantibodies (NAAb) (Coutinho et al. 1995). Levels of NAAb in blood were found to be related to neurological changes caused by stress in humans (Abou-Donia et al. 2013), and therefore could be a biomarker to measure the effects of stress on innate immunity. More research is needed to investigate whether enrichment also affects levels of (auto)antibodies other than KLH in pigs, and whether this may have consequences for their health.

In conclusion, there are several indications that environmental enrichment with substrates has a beneficial effect on the biological functioning of pigs, as pigs in barren housing seem to suffer more from chronic stress. Effects on the immune system and on susceptibility to infectious disease are less wellstudied and deserve further attention.

\subsection{Effects of early life and (mis)match of early and later life environments}

As indicated above, absence or presence of substrates as environmental enrichment for pigs seems to influence all three constituents of animal welfare, although studies on the impact on affective state and immune function are less abundant that those focusing on natural behaviour. Part of the effects of enrichment may derive from developmental changes caused by early life experience with environmental enrichment, which may have effects on later life functioning. Moreover, given the fact that most pigs are 
relocated at least once during their lives, it is vital to consider the impact of a change in environment, potentially causing a mismatch between the early life and current housing conditions that they are exposed to.

It has been shown that early life experiences may have long-term impacts on behaviour, affective state, and immunity via different ways, as during early life phases brain circuits are highly plastic, and the organism seems to be most susceptible to external influence (Champagne and Curley 2005). Most of the studies on early life experiences focus on early life stress, which is defined as any condition including an adverse environment or experience, and eliciting activation of the HPA axis (Maniam et al. 2014; Meaney et al. 1994). The HPA axis plays a critical role in modulating the adaptation and response to later stressors (Maniam et al. 2014), and is an important regulator of behaviour (including cognitive abilities), mental state and immune function (Bellavance and Rivest 2014; O'Mahony et al. 2009; Sachser et al. 2013). Besides, the early life environment can influence gut microbiota development, which, in turn, may alter brain functions via the gut-brain axis. The gut-brain axis is a bidirectional communication network, and composed of several routes including hormonal, immune and neuronal pathways (Clarke et al. 2014). The gut-brain axis influences behaviour (Clarke et al. 2014), affective state (Evrensel and Ceylan 2015; Foster and Neufeld 2013) and immune function (El Aidy et al. 2014).

Early life experiences have been found to modulate the development of behaviour, cognition and health in farm animals, including pigs, too (reviewed in Telkänranta and Edwards 2018). For example, the rearing environment of piglets (group-farrowing system vs. confinement system) 
affected agonistic behaviours of growing pigs during mixing ( $\mathrm{Li}$ and Wang 2011). In addition, it has also been shown that the influence of early weaning stress on intestinal barrier function, immunity and nervous system function persisted into adulthood in pigs (Medland et al. 2016; Pohl et al. 2017; Smith et al. 2009).

Pigs may experience both different levels of stress and hygienic conditions when reared in barren housing as opposed to housing enriched with substrates. Different housing experiences with or without enrichment may therefore have long-term effects as well. Indeed, there is some evidence for early life enrichment to affect later life behaviour and HPA axis functioning, as reflected in altered cortisol concentrations (Chaloupková et al. 2007; Munsterhjelm et al. 2009; Munsterhjelm et al. 2010). Little is known about the long-term effects of early life environmental enrichment on affective states and immunity. Other factors in early life were, however, found to influence immunity in pigs. For example, in new-born piglets, early life environmental variation (antibiotic treatment and stressful management procedures) affected intestinal microbiota colonization and immune development (Schokker et al. 2014). Maternal deprivation and social isolation, along with enhancing basal ACTH concentrations and glucocorticoid receptor binding in the hippocampus, increased IL- $\beta$ content (Kanitz et al. 2004). However, studies in other species have shown that early life experiences do not appear to affect all elements of the immune system to the same extent (Elwenspoek et al. 2017).

The effects of presence or absence of enrichment materials in the current environment may depend on the early life experiences of pigs, especially if 
the quality of the environments during early life rearing and current environment do not match. Day et al. (2002) found that the beneficial effects of enrichment on behaviour in pigs were more pronounced if these pigs had also been provided with substrates in early life, i.e. in case of a match between their early and later life environment. If early life and current housing do not match with respect to the availability of enrichment substrates, different types of effect of this mismatch are conceivable. Firstly, rearing pigs in barren pens may disturb normal behavioural development, leading to damaging behaviours that persist even when they are placed in a more enriched environment (reviewed in Telkänranta and Edwards 2018). Conversely, studies in other species have shown current environmental enrichment can lead to a functional reversal of the effects of adverse early life experiences on behaviour and mental functions (Francis et al. 2002; Koh et al. 2005). This suggests that the negative effects of being reared in barren housing could be reversed by providing enrichment. Also, a change from enriched early life housing to barren pens may have implications for welfare. Apart from affecting development, early life experiences may have an influence on how pigs perceive and value the features in their later life (Oostindjer et al. 2011). It has been reported that switching pigs from enriched to barren pens may exacerbate redirection of explorative behaviours to towards pen fixtures and pen mates as compared with barren reared pigs, indicating a decrease in welfare in the pigs that experienced a loss of enrichment (Munsterhjelm et al. 2009). Furthermore, barren housed pigs that had spent time in an enriched environment for a short period before, reacted more pessimistically in a judgement bias test, likely reflecting a more negative affective state in those pigs (Douglas et al. 2012). Taken together, 
this suggests that loss of enrichment may be more detrimental and frustrating for pigs than having no enrichment substrates at all. This is opposite to studies suggesting that enrichment in early life enhances adaptive capacity in animals and protects them from developing abnormal behaviours. For instance, mice reared in enriched cages before had less stereotypic behaviours when switched to standard cages than those reared in standard cages throughout (Hadley et al. 2006; Powell et al. 2000).

In conclusion, there are indications that the impact of current housing conditions of pigs on their welfare may depend on their early life environment, and vice versa, but more research is needed to pinpoint the effects on behaviour and affective state. Whether early life enrichment and a (mis)match between the level enrichment during development and in later life also affect immunity and health in pigs is largely unknown and also needs further study.

\subsection{Aims and outlines of this thesis}

It is becoming more and more clear that environmental enrichment affects the behaviour of pigs, but there is limited evidence to show the impact on mental and physical health. Besides, the long-term effects of early life enrichment and of a (mis)match with the current environment on pigs are less known.

The main objective of the current thesis was therefore to investigate whether and how early life and current environmental enrichment affect behaviour, affective state, and immunity in pigs. 
To this aim, first the influence of environmental enrichment on natural (auto)antibodies (NA(A)b) binding danger (phosphorylcholine-conjugated to bovine serum albumin, PC-BSA) and neural antigens (myelin basic protein, MBP) in healthy pigs was studied in Chapter 2. Chapter 3 focuses on the effect of environmental enrichment on $N A(A) b$ in pigs co-infected with porcine reproductive and respiratory syndrome virus (PRRSV) and $A$. pleuropneumoniae. These two chapters thus investigated the effect of current enriched housing on the $N A(A) b$ levels in healthy and unhealthy pigs.

To further investigate the effects of early life enrichment and a (mis)match in early and later life housing on behaviour, affective state, and immunity in pigs, an experiment was conducted in which pigs were housed in either barren or enriched pens from birth, with half of them experiencing a housing switch at 7 weeks of age. Chapter 4 describes the effects of early life and current housing on the development of behaviours in pigs, focusing on damaging, manipulative, explorative, social and play behaviours. An attention bias test and a successive negative contrast test were used in Chapter 5 and 6, respectively, to assess the effects of early life and current housing conditions on (negative) affective states in pigs. To study the effects of early life and current housing conditions on innate and adaptive immune competence, pigs were immunized with a model antigen (keyhole limpet hemocyanin-conjugated trinitrophenyl, KLH-TNP) twice to induce primary and secondary antigen-specific antibody responses. The levels of specific antibodies binding with this antigen in blood and peripheral blood leukocyte sub-populations were analysed in Chapter 7. 
Finally, in Chapter 8 the findings from the previous chapters are discussed and integrated, also including results of a number of fear tests the pigs were exposed to in this project (not shown in a separate chapter). Besides, in this chapter, a first attempt is made to identify putative relationships between behaviour, affective state, and immunity in pigs.

The coping style of the pigs was taken into account in most chapters of this thesis, as this trait is considered to be important in the explanation of individual variation in immunity (reviewed in Koolhaas 2008). Besides, it has been suggested that the impact of environment and a change in environment on behaviour and affective state may differ for pigs with diverging coping styles (Asher et al. 2016; Bolhuis et al. 2005; Ruis et al. 2000). 


\subsection{References:}

Abou-Donia MB, Abou-Donia MM, EIMasry EM, Monro JA, Mulder MF (2013) Autoantibodies to nervous system-specific proteins are elevated in sera of flight crew members: biomarkers for nervous system injury Journal of Toxicology and Environmental Health, Part A 76:363-380

Anisman H, Merali Z (2003) Cytokines, stress and depressive illness: brainimmune interactions Annals of medicine 35:2-11

Arai JA, Feig LA (2011) Long-lasting and transgenerational effects of an environmental enrichment on memory formation Brain research bulletin 85:30-35

Archard GA, Earley RL, Hanninen AF, Braithwaite VA (2012) Correlated behaviour and stress physiology in fish exposed to different levels of predation pressure Functional Ecology 26:637-645

Asher L, Friel M, Griffin K, Collins LM (2016) Mood and personality interact to determine cognitive biases in pigs Biology letters 12:20160402

Ball N, Mercado E, Orduña I (2019) Enriched Environments as a Potential Treatment for Developmental Disorders: A Critical Assessment Frontiers in psychology 10:466

Bammert J et al. (1993) An ethological approach to animal-welfare-the supply of needs and avoidance of damage Tierarztliche Umschau 48:269

Banks WA, Erickson MA (2010) The blood-brain barrier and immune function and dysfunction Neurobiology of disease 37:26-32

Barak Y (2006) The immune system and happiness Autoimmunity reviews 5:523-527

Barnett JL, Hemsworth PH, Cronin GM, Jongman EC, Hutson G (2001) A review of the welfare issues for sows and piglets in relation to housing Australian journal of agricultural research 52:1-28

Baroncelli L, Braschi C, Spolidoro M, Begenisic T, Sale A, Maffei L (2010) Nurturing brain plasticity: impact of environmental enrichment Cell death and differentiation 17:1092

Barrett LF (1998) Discrete emotions or dimensions? The role of valence focus and arousal focus Cognition \& Emotion 12:579-599

Bateson M, Matheson S (2007) Performance on a categorisation task suggests that removal of environmental enrichment inducespessimism'in captive European starlings (Sturnus vulgaris) Animal welfare-potters bar then wheathampstead 16:33 
Beattie V, O'connell N, Kilpatrick D, Moss B (2000a) Influence of environmental enrichment on welfare-related behavioural and physiological parameters in growing pigs Animal Science 70:443-450

Beattie V, O'connell N, Moss B (2000b) Influence of environmental enrichment on the behaviour, performance and meat quality of domestic pigs Livestock production science 65:71-79

Beattie V, Walker N, Sneddon I (1995) Effects of environmental enrichment on behaviour and productivity of growing pigs Animal Welfare 4:207220

Beattie V, Walker N, Sneddon I (1996) An investigation of the effect of environmental enrichment and space allowance on the behaviour and production of growing pigs Applied animal behaviour science 48:151-158

Becker B, Nienaber J, Christenson R, Manak R, DeShazer J, Hahn G (1985) Peripheral concentrations of cortisol as an indicator of stress in the pig American Journal of Veterinary Research 46:1034-1038

Bellavance M-A, Rivest S (2014) The HPA-immune axis and the immunomodulatory actions of glucocorticoids in the brain Frontiers in immunology 5:136

Boissy A et al. (2007) Emotions and cognition: a new approach to animal welfare Animal Welfare 16:37-43

Bolhuis JE, Oostindjer M, Hoeks CW, de Haas EN, Bartels AC, Ooms M, Kemp B (2013) Working and reference memory of pigs (Sus scrofa domesticus) in a holeboard spatial discrimination task: the influence of environmental enrichment Animal cognition 16:845-850

Bolhuis JE, Schouten WG, Schrama JW, Wiegant VM (2005) Behavioural development of pigs with different coping characteristics in barren and substrate-enriched housing conditions Applied Animal Behaviour Science 93:213-228

Bolhuis JE, Schouten WG, Schrama JW, Wiegant VM (2006) Effects of rearing and housing environment on behaviour and performance of pigs with different coping characteristics Applied Animal Behaviour Science 101:68-85

Bracke MB, Koene $P$ (2019) Expert opinion on metal chains and other indestructible objects as proper enrichment for intensively-farmed pigs PloS one 14:e0212610

Bradley BP, Mogg K, Lee SC (1997) Attentional biases for negative information in induced and naturally occurring dysphoria Behaviour research and therapy 35:911-927 
Bradley BP, Mogg K, Millar N, White J (1995) Selective processing of negative information: Effects of clinical anxiety, concurrent depression, and awareness Journal of abnormal psychology 104:532

Brydges NM, Leach M, Nicol K, Wright R, Bateson M (2011) Environmental enrichment induces optimistic cognitive bias in rats Animal Behaviour 81:169-175

Burman OH, Parker RM, Paul ES, Mendl M (2008) Sensitivity to reward loss as an indicator of animal emotion and welfare Biology letters 4:330333

Caldara FR et al. (2012) Behavior, performance and physiological parameters of pigs reared in deep bedding Engenharia Agrícola 32:38-46

Camerlink I, Bijma P, Kemp B, Bolhuis JE (2012) Relationship between growth rate and oral manipulation, social nosing, and aggression in finishing pigs Applied Animal Behaviour Science 142:11-17

Capuron L, Miller AH (2011) Immune system to brain signaling: neuropsychopharmacological implications Pharmacology \& therapeutics 130:226-238

Carreras R et al. (2016) Housing conditions do not alter cognitive bias but affect serum cortisol, qualitative behaviour assessment and wounds on the carcass in pigs Applied Animal Behaviour Science 185:39-44

Chaby L, Cavigelli S, White A, Wang K, Braithwaite V (2013) Long-term changes in cognitive bias and coping response as a result of chronic unpredictable stress during adolescence Frontiers in human neuroscience 7:328

Chaloupková H, Illmann G, Neuhauserová K, Tománek M, Valis L (2007) Preweaning housing effects on behavior and physiological measures in pigs during the suckling and fattening periods Journal of animal science 85:1741-1749

Chamove AS (1989) Environmental enrichment: a review Anim Technol 40:155-178

Champagne FA, Curley JP (2005) How social experiences influence the brain Current opinion in neurobiology 15:704-709

Chiang JJ, Taylor SE, Bower JE (2015) Early adversity, neural development, and inflammation Developmental psychobiology 57:887-907

Clarke G, O'Mahony S, Dinan T, Cryan J (2014) Priming for health: gut microbiota acquired in early life regulates physiology, brain and behaviour Acta Paediatrica 103:812-819

Coutinho A, Kazatchkine MD, Avrameas S (1995) Natural autoantibodies Current opinion in immunology 7:812-818 
Dalmau A, Fabrega E, Velarde A (2009) Fear assessment in pigs exposed to a novel object test Applied Animal Behaviour Science 117:173-180

Dantzer R (2004) Innate immunity at the forefront of psychoneuroimmunology Brain, behavior, and immunity 18:1-6

Dantzer R (2017) Neuroimmune interactions: from the brain to the immune system and vice versa Physiological reviews 98:477-504

Dawkins MS (1988) Behavioural deprivation: a central problem in animal welfare Applied Animal Behaviour Science 20:209-225

Day J, Burfoot A, Docking C, Whittaker X, Spoolder H, Edwards S (2002) The effects of prior experience of straw and the level of straw provision on the behaviour of growing pigs Applied Animal Behaviour Science 76:189-202

de Groot J, de Jong IC, Prelle IT, Koolhaas JM (2000) Immunity in barren and enriched housed pigs differing in baseline cortisol concentration Physiology \& behavior 71:217-223

de Jong IC et al. (1998) Effects of strawbedding on physiological responses to stressors and behavior in growing pigs Physiology \& behavior 64:303310

de Jong IC, Prelle IT, van de Burgwal JA, Lambooij E, Korte SM, Blokhuis HJ, Koolhaas JM (2000) Effects of environmental enrichment on behavioral responses to novelty, learning, and memory, and the circadian rhythm in cortisol in growing pigs Physiology \& behavior 68:571-578

De Jonge FH, Bokkers E, Schouten W, Helmond F (1996) Rearing piglets in a poor environment: developmental aspects of social stress in pigs Physiology \& Behavior 60:389-396

Douglas C, Bateson M, Walsh C, Bédué A, Edwards SA (2012) Environmental enrichment induces optimistic cognitive biases in pigs Applied Animal Behaviour Science 139:65-73

Dudink S, Simonse H, Marks I, de Jonge FH, Spruijt BM (2006) Announcing the arrival of enrichment increases play behaviour and reduces weaningstress-induced behaviours of piglets directly after weaning Applied Animal Behaviour Science 101:86-101

Duncan IJ (2005) Science-based assessment of animal welfare: farm animals Revue scientifique et technique-Office international des epizooties 24:483

Edwards S (2006) Tail biting in pigs: understanding the intractable problem The Veterinary Journal 171:198-199 
El Aidy S, Dinan TG, Cryan JF (2014) Immune modulation of the brain-gutmicrobe axis Frontiers in microbiology 5:146

Elwenspoek MM, Kuehn A, Muller CP, Turner JD (2017) The effects of early life adversity on the immune system Psychoneuroendocrinology 82:140-154

Ernst K, Tuchscherer M, Kanitz E, Puppe B, Manteuffel G (2006) Effects of attention and rewarded activity on immune parameters and wound healing in pigs Physiology \& behavior 89:448-456

Evrensel A, Ceylan ME (2015) The gut-brain axis: the missing link in depression Clinical Psychopharmacology and Neuroscience 13:239

Felten S, Olschowka J (1987) Noradrenergic sympathetic innervation of the spleen: II. Tyrosine hydroxylase (TH)-positive nerve terminals form synapticlike contacts on lymphocytes in the splenic white pulp Journal of neuroscience research 18:37-48

Forsythe P, Bienenstock J, Kunze WA (2014) Vagal pathways for microbiomebrain-gut axis communication. In: Microbial Endocrinology: The Microbiota-Gut-Brain Axis in Health and Disease. Springer, pp 115133

Foster JA, Neufeld K-AM (2013) Gut-brain axis: how the microbiome influences anxiety and depression Trends in neurosciences 36:305312

Francis DD, Diorio J, Plotsky PM, Meaney MJ (2002) Environmental enrichment reverses the effects of maternal separation on stress reactivity Journal of Neuroscience 22:7840-7843

Franks B (2018) Cognition as a cause, consequence, and component of welfare. In: Advances in Agricultural Animal Welfare. Elsevier, pp 324

Fraser D (2008a) Animal welfare and the intensification of animal production. In: The ethics of intensification. Springer, pp 167-189

Fraser D (2008b) Understanding animal welfare Acta Veterinaria Scandinavica 50:S1

Fraser D, Phillips P, Thompson B, Tennessen T (1991) Effect of straw on the behaviour of growing pigs Applied Animal Behaviour Science 30:307318

Fraser D, Weary DM, Pajor EA, Milligan BN (1997) A scientific conception of animal welfare that reflects ethical concerns

Gentry J, McGlone J, Blanton Jr J, Miller M (2002) Alternative housing systems for pigs: Influences on growth, composition, and pork quality Journal of animal science $80: 1781-1790$ 
Grimberg-Henrici CG, Vermaak P, Bolhuis JE, Nordquist RE, van der Staay FJ (2016) Effects of environmental enrichment on cognitive performance of pigs in a spatial holeboard discrimination task Animal cognition 19:271-283

Guy J, Rowlinson P, Chadwick J, Ellis M (2002) Health conditions of two genotypes of growing-finishing pig in three different housing systems: implications for welfare Livestock Production Science 75:233-243

Hadley C, Hadley B, Ephraim S, Yang M, Lewis MH (2006) Spontaneous stereotypy and environmental enrichment in deer mice (Peromyscus maniculatus): reversibility of experience Applied Animal Behaviour Science 97:312-322

Held SD, Špinka M (2011) Animal play and animal welfare Animal behaviour 81:891-899

Jansen J, Bolhuis JE, Schouten WG, Spruijt BM, Wiegant VM (2009) Spatial learning in pigs: effects of environmental enrichment and individual characteristics on behaviour and performance Animal cognition 12:303-315

Janssens CJ, Helmond FA, Wiegant VM (1994) Increased cortisol response to exogenous adrenocorticotropic hormone in chronically stressed pigs: influence of housing conditions Journal of Animal Science 72:17711777

Jiang C-L, Lu C-L, Liu X-Y (1998) The molecular basis for bidirectional communication between the immune and neuroendocrine systems Domestic animal endocrinology 15:363-369

Jirkof P (2015) Effects of experimental housing conditions on recovery of laboratory mice Lab animal 44:65

Jordan D, Žgur S, Gorjanc G, Štuhec I (2008) Straw or hay as environmental improvement and its effect on behaviour and production traits of fattening pigs Archives Animal Breeding 51:549-559

Kallio P, Janczak A, Valros A, Edwards S, Heinonen M (2018) Case control study on environmental, nutritional and management-based risk factors for tail-biting in long-tailed pigs Animal Welfare

Kanitz E, Tuchscherer M, Puppe B, Tuchscherer A, Stabenow B (2004) Consequences of repeated early isolation in domestic piglets (Sus scrofa) on their behavioural, neuroendocrine, and immunological responses Brain, Behavior, and Immunity 18:35-45

Kiecolt-Glaser JK, Gouin J-P, Weng N-p, Malarkey WB, Beversdorf DQ, Glaser R (2011) Childhood adversity heightens the impact of later-life 
caregiving stress on telomere length and inflammation Psychosomatic medicine 73:16

Klont R et al. (2001) Relationships between behavioral and meat quality characteristics of pigs raised under barren and enriched housing conditions Journal of Animal Science 79:2835-2843

Koh S, Chung H, Xia H, Mahadevia A, Song Y (2005) Environmental enrichment reverses the impaired exploratory behavior and altered gene expression induced by early-life seizures Journal of child neurology 20:796-802

Koolhaas J (2008) Coping style and immunity in animals: making sense of individual variation Brain, behavior, and immunity 22:662-667

Kusnecov AW, Rabin BS (1994) Stressor-Induced Alterations of Immune Function: Mechanisms and Issues International archives of allergy and immunology 105:107-121

Lammers A, Klomp ME, Nieuwland MG, Savelkoul HF, Parmentier HK (2004) Adoptive transfer of natural antibodies to non-immunized chickens affects subsequent antigen-specific humoral and cellular immune responses Developmental \& Comparative Immunology 28:51-60

Lee C, Cafe LM, Robinson SL, Doyle RE, Lea JM, Small AH, Colditz IG (2018) Anxiety influences attention bias but not flight speed and crush score in beef cattle Applied animal behaviour science 205:210-215

Lee C, Verbeek E, Doyle R, Bateson M (2016) Attention bias to threat indicates anxiety differences in sheep Biology letters 12:20150977

Leggio MG, Mandolesi L, Federico F, Spirito F, Ricci B, Gelfo F, Petrosini L (2005) Environmental enrichment promotes improved spatial abilities and enhanced dendritic growth in the rat Behavioural brain research 163:78-90

Li Y, Wang L (2011) Effects of previous housing system on agonistic behaviors of growing pigs at mixing Applied Animal Behaviour Science 132:2026

Lukkes JL, Mokin MV, Scholl JL, Forster GL (2009) Adult rats exposed to earlylife social isolation exhibit increased anxiety and conditioned fear behavior, and altered hormonal stress responses Hormones and behavior 55:248-256

Lyons C, Bruce J, Fowler V, English P (1995) A comparison of productivity and welfare of growing pigs in four intensive systems Livestock production science 43:265-274 
Maniam J, Antoniadis C, Morris MJ (2014) Early-life stress, HPA axis adaptation, and mechanisms contributing to later health outcomes Frontiers in endocrinology 5:73

Manson JJ, Mauri C, Ehrenstein MR Natural serum IgM maintains immunological homeostasis and prevents autoimmunity. In: Springer seminars in immunopathology, 2005. vol 4. Springer, pp 425-432

Markowitz H, Gavazzi A (1995) Eleven principles for improving the quality of captive animal life LAB ANIMAL-NEW YORK- 24:30-30

Mason G, Walker M, Duggan G, Roulston N, Van Slack A (2012) Negative affective states and their effects on morbidity, mortality and longevity

Matheson SM, Asher L, Bateson M (2008) Larger, enriched cages are associated with 'optimistic'response biases in captive European starlings (Sturnus vulgaris) Applied Animal Behaviour Science 109:374-383

Meaney MJ et al. Early environmental programming hypothalamic-pituitaryadrenal responses to stress. In: Seminars in Neuroscience, 1994. vol 4. Elsevier, pp 247-259

Medland JE, Pohl CS, Edwards LL, Frandsen S, Bagley K, Li Y, Moeser AJ (2016) Early life adversity in piglets induces long-term upregulation of the enteric cholinergic nervous system and heightened, sex-specific secretomotor neuron responses Neurogastroenterology \& Motility 28:1317-1329

Meehan C, Mench J (2002) Environmental enrichment affects the fear and exploratory responses to novelty of young Amazon parrots Applied Animal Behaviour Science 79:75-88

Mench JA (1994) Environmental enrichment and exploration Lab Anim(NY) 23:38-41

Mendl M (1999) Performing under pressure: stress and cognitive function Applied Animal Behaviour Science 65:221-244

Mendl M, Burman OH, Parker RM, Paul ES (2009) Cognitive bias as an indicator of animal emotion and welfare: Emerging evidence and underlying mechanisms Applied Animal Behaviour Science 118:161181

Mendl M, Burman OH, Paul ES (2010) An integrative and functional framework for the study of animal emotion and mood Proceedings of the Royal Society B: Biological Sciences 277:2895-2904 
Merlot E et al. (2012) Health and immune traits of Basque and Large White pigs housed in a conventional or enriched environment Animal 6:1290-1299

Mkwanazi MV, Ncobela CN, Kanengoni AT, Chimonyo M (2019) Effects of environmental enrichment on behaviour, physiology and performance of pigs-A review Asian-Australasian journal of animal sciences 32:1

Moinard C, Mendl M, Nicol C, Green L (2003) A case control study of on-farm risk factors for tail biting in pigs Applied Animal Behaviour Science 81:333-355

Monk JE, Doyle RE, Colditz IG, Belson S, Cronin GM, Lee C (2018) Towards a more practical attention bias test to assess affective state in sheep PLoS One 13:e0190404

Munsterhjelm C, Peltoniemi OA, Heinonen M, Hälli O, Karhapää M, Valros A (2009) Experience of moderate bedding affects behaviour of growing pigs Applied Animal Behaviour Science 118:42-53

Munsterhjelm C, Valros A, Heinonen M, Hälli O, Siljander-Rasi H, Peltoniemi O (2010) Environmental enrichment in early life affects cortisol patterns in growing pigs Animal 4:242-249

Murphy E, Nordquist RE, van der Staay FJ (2014) A review of behavioural methods to study emotion and mood in pigs, Sus scrofa Applied Animal Behaviour Science 159:9-28

Newberry RC (1995) Environmental enrichment: increasing the biological relevance of captive environments Applied Animal Behaviour Science 44:229-243

Nicol C (1996) Farm animal cognition Animal Science 62:375-391

O'Mahony SM et al. (2009) Early life stress alters behavior, immunity, and microbiota in rats: implications for irritable bowel syndrome and psychiatric illnesses Biological psychiatry 65:263-267

Oliveira R et al. (2016) Environmental enrichment improves the performance and behavior of piglets in the nursery phase Arquivo Brasileiro de Medicina Veterinária e Zootecnia 68:415-421

Oostindjer M, Bolhuis J, Mendl M, Held S, Gerrits W, Van den Brand H, Kemp $B$ (2010) Effects of environmental enrichment and loose housing of lactating sows on piglet performance before and after weaning Journal of Animal Science 88:3554-3562

Oostindjer M, van den Brand H, Kemp B, Bolhuis JE (2011) Effects of environmental enrichment and loose housing of lactating sows on 
piglet behaviour before and after weaning Applied Animal Behaviour Science 134:31-41

Paul ES, Harding EJ, Mendl M (2005) Measuring emotional processes in animals: the utility of a cognitive approach Neuroscience \& Biobehavioral Reviews 29:469-491

Pedersen $\sqcup$ (2018) Overview of commercial pig production systems and their main welfare challenges. In: Advances in Pig Welfare. Elsevier, pp 325

Peeters E, Driessen B, Moons CP, Ödberg FO, Geers R (2006) Effect of temporary straw bedding on pigs' behaviour, performance, cortisol and meat quality Applied animal behaviour science 98:234-248

Petersen V, Simonsen HB, Lawson LG (1995) The effect of environmental stimulation on the development of behaviour in pigs Applied Animal Behaviour Science 45:215-224

Pohl CS et al. (2017) Early weaning stress induces chronic functional diarrhea, intestinal barrier defects, and increased mast cell activity in a porcine model of early life adversity Neurogastroenterology \& Motility 29:e13118

Powell SB, Newman HA, McDonald TA, Bugenhagen P, Lewis MH (2000) Development of spontaneous stereotyped behavior in deer mice: effects of early and late exposure to a more complex environment Developmental Psychobiology: The Journal of the International Society for Developmental Psychobiology 37:100-108

Quartier P, Potter PK, Ehrenstein MR, Walport MJ, Botto M (2005) Predominant role of IgM-dependent activation of the classical pathway in the clearance of dying cells by murine bone marrowderived macrophages in vitro European journal of immunology 35:252-260

Randolph J, Cromwell G, Stahly T, Kratzer D (1981) Effects of group size and space allowance on performance and behavior of swine Journal of Animal Science 53:922-927

Rauterberg $S$ et al. (2013) Cognitive enrichment in the farrowing pen-a first approach to use early behavioural conditioning of suckling piglets to reduce aggressive behaviour during rearing Züchtungskunde 85:376387

Reimert I, Rodenburg TB, Ursinus WW, Duijvesteijn N, Camerlink I, Kemp B, Bolhuis JE (2013) Backtest and novelty behavior of female and castrated male piglets, with diverging social breeding values for growth Journal of animal science 91:4589-4597 
Reimert I, Rodenburg TB, Ursinus WW, Kemp B, Bolhuis JE (2014a) Responses to novel situations of female and castrated male pigs with divergent social breeding values and different backtest classifications in barren and straw-enriched housing Applied animal behaviour science 151:24-35

Reimert I, Rodenburg TB, Ursinus WW, Kemp B, Bolhuis JE (2014b) Selection based on indirect genetic effects for growth, environmental enrichment and coping style affect the immune status of pigs PloS one 9:e108700

Renner MJ, Rosenzweig MR (1987) Enriched and impoverished environments: Effects on brain and behavior. Springer,

Richard S, Wacrenier-Ceré N, Hazard D, Saint-Dizier H, Arnould C, Faure J (2008) Behavioural and endocrine fear responses in Japanese quail upon presentation of a novel object in the home cage Behavioural Processes 77:313-319

Riemer S, Ellis SL, Ryan S, Thompson H, Burman OH (2016) A reappraisal of successive negative contrast in two populations of domestic dogs Animal cognition 19:471-481

Roelofs S, Boleij H, Nordquist RE, van der Staay FJ (2016) Making decisions under ambiguity: judgment bias tasks for assessing emotional state in animals Frontiers in behavioral neuroscience 10:119

Romeo RD, Mueller A, Sisti HM, Ogawa S, McEwen BS, Brake WG (2003) Anxiety and fear behaviors in adult male and female C57BL/6 mice are modulated by maternal separation Hormones and behavior 43:561-567

Romeyer A, Bouissou M-F (1992) Assessment of fear reactions in domestic sheep, and influence of breed and rearing conditions Applied Animal Behaviour Science 34:93-119

Rosenzweig MR, Bennett EL (1996) Psychobiology of plasticity: effects of training and experience on brain and behavior Behavioural brain research 78:57-65

Ruis MA, te Brake JH, van de Burgwal JA, de Jong IC, Blokhuis HJ, Koolhaas JM (2000) Personalities in female domesticated pigs: behavioural and physiological indications Applied Animal Behaviour Science 66:31-47

Sachser N, Kaiser S, Hennessy MB (2013) Behavioural profiles are shaped by social experience: when, how and why Philosophical Transactions of the Royal Society B: Biological Sciences 368:20120344 
Schlegelmilch T, Henke K, Peri F (2011) Microglia in the developing brain: from immunity to behaviour Current opinion in neurobiology 21:510

Schmeer KK, Ford JL, Browning CR (2019) Early childhood family instability and immune system dysregulation in adolescence Psychoneuroendocrinology 102:189-195

Schnall S (2010) Affect, mood and emotions Social and emotional aspect of learning:59-64

Schokker D et al. (2014) Early-life environmental variation affects intestinal microbiota and immune development in new-born piglets PLoS One 9:e100040

Schrijver NC, Bahr NI, Weiss IC, Würbel H (2002) Dissociable effects of isolation rearing and environmental enrichment on exploration, spatial learning and HPA activity in adult rats Pharmacology Biochemistry and Behavior 73:209-224

Scollo A, Contiero B, Gottardo F (2016) Frequency of tail lesions and risk factors for tail biting in heavy pig production from weaning to $170 \mathrm{~kg}$ live weight The Veterinary Journal 207:92-98

Scott $\mathrm{K}$ et al. (2006) The welfare of finishing pigs in two contrasting housing systems: Fully-slatted versus straw-bedded accommodation Livestock Science 103:104-115

Shepherdson D (1998) Tracing the path of environmental enrichment in zoos Second nature: Environmental enrichment for captive animals:1-12

Sheriff MJ, Krebs CJ, Boonstra R (2009) The sensitive hare: sublethal effects of predator stress on reproduction in snowshoe hares Journal of Animal Ecology 78:1249-1258

Smith F et al. (2009) Early weaning stress impairs development of mucosal barrier function in the porcine intestine American Journal of Physiology-Gastrointestinal and Liver Physiology 298:G352-G363

Sneddon I, Beattie V, Dunne L, Neil W (2000) The effect of environmental enrichment on learning in pigs Animal Welfare 9:373-383

Sonoda LT et al. (2013) Cognitive enrichment in piglet rearing: an approach to enhance animal welfare and to reduce aggressive behaviour ISRN veterinary science 2013

Spinka M, Newberry RC, Bekoff M (2001) Mammalian play: training for the unexpected The quarterly review of biology 76:141-168

Sternberg EM (2006) Neural regulation of innate immunity: a coordinated nonspecific host response to pathogens Nature Reviews Immunology 6:318 
Studnitz M, Jensen MB, Pedersen LJ (2007) Why do pigs root and in what will they root?: A review on the exploratory behaviour of pigs in relation to environmental enrichment Applied animal behaviour science 107:183-197

Taylor NR, Main DC, Mendl M, Edwards SA (2010) Tail-biting: a new perspective The Veterinary Journal 186:137-147

Taylor NR, Parker RM, Mendl M, Edwards SA, Main DC (2012) Prevalence of risk factors for tail biting on commercial farms and intervention strategies The Veterinary Journal 194:77-83

Telkänranta H, Edwards SA (2018) Lifetime consequences of the early physical and social environment of piglets. In: Advances in Pig Welfare. Elsevier, pp 101-136

Tucker DM, Luu P (2007) Neurophysiology of motivated learning: adaptive mechanisms underlying cognitive bias in depression Cognitive Therapy and Research 31:189-209

Turner S, Ewen M, Rooke J, Edwards S (2000) The effect of space allowance on performance, aggression and immune competence of growing pigs housed on straw deep-litter at different group sizes Livestock Production Science 66:47-55

Tuyttens FAM (2005) The importance of straw for pig and cattle welfare: a review Applied animal behaviour science 92:261-282

Ursinus WW, Van Reenen CG, Kemp B, Bolhuis JE (2014) Tail biting behaviour and tail damage in pigs and the relationship with general behaviour: Predicting the inevitable? Applied Animal Behaviour Science 156:2236

van de Weerd H, Ison S (2019) Providing Effective Environmental Enrichment to Pigs: How Far Have We Come? Animals 9:254

van de Weerd HA, Day JE (2009) A review of environmental enrichment for pigs housed in intensive housing systems Applied Animal Behaviour Science 116:1-20

Van de Weerd HA, Docking CM, Day JE, Avery PJ, Edwards SA (2003) A systematic approach towards developing environmental enrichment for pigs Applied Animal Behaviour Science 84:101-118

van Dixhoorn ID et al. (2016) Enriched housing reduces disease susceptibility to co-infection with porcine reproductive and respiratory virus (PRRSV) and Actinobacillus pleuropneumoniae (A. pleuropneumoniae) in young pigs PloS one 11:e0161832

Van Praag H, Kempermann G, Gage FH (2000) Neural consequences of enviromental enrichment Nature Reviews Neuroscience 1:191 
Van Reenen C et al. (2009) The benzodiazepine brotizolam reduces fear in calves exposed to a novel object test Physiology \& behavior 96:307314

Varman DR, Marimuthu G, Rajan KE (2012) Environmental enrichment exerts anxiolytic effects in the Indian field mouse (Mus booduga) Applied animal behaviour science 136:166-173

Veena J, Srikumar B, Mahati K, Bhagya V, Raju T, Shankaranarayana Rao B (2009) Enriched environment restores hippocampal cell proliferation and ameliorates cognitive deficits in chronically stressed rats Journal of neuroscience research 87:831-843

Webster J (2008) Animal Welfare: limping towards eden: A practical approach to redressing the problem of our dominion over the animals. John Wiley \& Sons,

Wemelsfelder F, Haskell M, Mendl MT, Calvert S, Lawrence AB (2000) Diversity of behaviour during novel object tests is reduced in pigs housed in substrate-impoverished conditions Animal Behaviour 60:385-394

Widowski TM, Torrey S, Bench CJ, Gonyou HW (2008) Development of ingestive behaviour and the relationship to belly nosing in earlyweaned piglets Applied animal behaviour science 110:109-127

Wohleb ES, McKim DB, Sheridan JF, Godbout JP (2015) Monocyte trafficking to the brain with stress and inflammation: a novel axis of immuneto-brain communication that influences mood and behavior Frontiers in neuroscience 8:447

Yang C-H, Ko H-L, Salazar LC, Llonch L, Manteca X, Camerlink I, Llonch P (2018) Pre-weaning environmental enrichment increases piglets' object play behaviour on a large scale commercial pig farm Applied Animal Behaviour Science 202:7-12

Zebunke M, Puppe B, Langbein J (2013) Effects of cognitive enrichment on behavioural and physiological reactions of pigs Physiology \& behavior 118:70-79

Zonderland JJ, Wolthuis-Fillerup M, Van Reenen CG, Bracke MB, Kemp B, Den Hartog LA, Spoolder HA (2008) Prevention and treatment of tail biting in weaned piglets Applied Animal Behaviour Science 110:269281 


\section{Chapter 2}

Effects of environmental enrichment and regrouping on natural autoantibodies-binding danger and neural antigens in healthy pigs with different individual characteristics

L. Luo, R. Geers, I. Reimert, B. Kemp, H. K. Parmentier, J. E. Bolhuis* Adaptation Physiology Group, Department of Animal Sciences, Wageningen University \& Research, P.O. Box 338, 6700 AH, The Netherlands

* Corresponding author: J. E. Bolhuis

E-mail address: liesbeth.bolhuis@wur.nl

Published: Animal (2017) 11.11: 2019-2026 


\subsection{Abstract}

Pigs living in commercial husbandry systems may experience both acute stress due to standard management procedures, and chronic stress through limitations in their barren housing environment. This might influence their immune status, including antibody responses to neural and danger autoantigens. Levels of natural auto-antibody (NAAb) binding phosphorylcholine (PC)-conjugated bovine serum albumin (BSA) and myelin basic protein (MBP) were measured over time in pigs that were kept in environmental enriched vs. barren housing, and that underwent a regrouping test. In total, 480 pigs were housed in 80 pens in either barren or straw-enriched pens from 4 through 23 weeks of age. Blood samples were taken from pigs before (week 8 ), and three days after a $24 \mathrm{~h}$ regrouping test (week 9), and at 22 weeks of age. PC-BSA and MBP antibody titers in serum were measured using ELISA. Enriched-housed pigs had higher levels of IgM binding MBP, and tended to have higher levels of IgG binding MBP and IgA binding PC-BSA than barrenhoused pigs. Each NAAb measured in this study was affected by gender and litter. These results suggest that enriched housing conditions, as well as acute regrouping stress, have an influence on levels of serum NAAb binding danger and neural antigens in pigs.

Keywords: environmental enrichment, natural autoantibody, pig, stress, immunity 


\subsection{Introduction}

Pigs in modern intensive husbandry systems can experience acute stress while being exposed to standard management procedures, such as tail docking, ear tagging and regrouping. The circumstances, under which pigs are kept, moreover, can lead to more sustained stress in these animals. Pigs in commercial husbandry usually live in barren, stimulus-poor conditions. The absence of suitable substrates for oral manipulation thwarts pigs from performing highly motivated behaviours such as rooting and chewing and may, therefore, induce chronic stress. Indeed, compared with pigs kept under more enriched conditions, barren-housed pigs show changes in hypothalamus-pituitary-adrenal (re)activity (de Jong et al. 2000), a decline in spatial cognitive performance (Grimberg-Henrici et al. 2016), suggesting impaired hippocampal functioning, and an increase in abnormal behaviours such as tail biting; e.g. (Camerlink et al. 2014). Moreover, barren housing also negatively affects mood of pigs, as reflected in a more pessimistic view of life (Douglas et al. 2012) and a decrease in play behaviours (Bolhuis et al. 2005). Part of the differences between animals in barren and enriched housing might be explained by cellular and molecular changes in the brain induced by environmental enrichment (Scholz et al. 2015).

There are indications for an effect of environmental enrichment as opposed to barren housing conditions on immune functions in pigs (Bolhuis et al. 2003). Both acute and chronic stress have been shown to result in altered immune functions (Dhabhar 2014). Also psychosocial factors can affect functioning of the immune system (Dhabhar 2014), as both a negative and a positive mood may trigger immune alterations (Koh 1998). Conversely, 
evidence is accumulating that mood and behaviour are affected by immune reactivity, including auto-immune responses towards brain and neuroendocrine structures (Maier and Watkins 1998).

Natural antibodies (NAb) are defined as immunoglobulins derived from selfrenewing CD5+ B-1 cells (Casali and Notkins 1989), without exogenous antigenic stimulation, and are found in all animal species tested so far (e.g. chickens (Sun et al. 2011), bovine (Mayasari et al. 2015) and wild boar (Rossi et al. 2013). NAb are important as first line of defence, and participate in physiological activities (Panda and Ding 2015). A large part of the NAb repertoire is directed to self-antigens, and these antibodies are referred to as natural auto-antibodies (NAAb). Blood levels of auto-antibodies binding neural antigens have been related to acute and chronic stress (Andrejević et al. 1997; Zhou et al. 1999), and could both reflect and affect neuronal functioning (Gold et al. 2012). Myelin basic protein (MBP) is an important protein in the process of myelination of nerves in the nervous system. And it has been found that high levels of auto-antibodies to MBP and other neural antigens were associated with neurological damage (Abou-Donia et al. 2013). Other studies reported relations between stress and auto-antibodies binding antigens reflecting cell damage and inflammation, such as phosphorylcholine (PC) (Lutz et al. 2009). PC is a component of cell membranes which is recognized by IgM autoantibodies after cell damage (Kim et al. 2002).

Previously, barren-housed and enriched-housed pigs were found to differ in levels of NAb binding the model antigen keyhole limpet hemocyanin (KLH) (Reimert et al. 2014b). However, effects of environmental enrichment on NAAb binding danger and neural antigens in healthy pigs (i.e. without clinical 
signs of illness) have not studied before. Our objective was to investigate the chronic effects of environmental enrichment (straw-enriched housing) vs. barren housing on levels of NAAb binding PC and MBP over time in pigs, and to study the effect of regrouping (inducing acute stress) on these autoantibodies. Individual characteristics of the pigs such as gender and personality, which influenced immune responses in other studies (Bolhuis et al. 2003; Reimert et al. 2014b) were taken into account as well.

\subsection{Materials and methods}

The established principles of laboratory animal use and care were followed, as well as the Dutch law on animal experiments. The Animal Care and Use Committee of Wageningen University \& Research approved the experiment.

\subsubsection{Animals and housing}

A total of 480 pigs, equally divided over five batches, were used in this study and were the same pigs as described in Reimert et al. (2014b). Briefly, pigs were born at the Topigs Norsvin experimental farm in Beilen, the Netherlands, where they were housed in conventional farrowing pens with their sow. On the day of weaning, at 28 days of age, pigs were transported to experimental farm 'de Haar' of Wageningen University \& Research, the Netherlands. Half of the pigs were housed in straw-enriched pens ( $\left.1 \mathrm{~m}^{2} / \mathrm{pig}\right)$ with $1.5 \mathrm{~kg}$ of straw and $12 \mathrm{~kg}$ of wood shavings ( $\mathrm{n}=40$ pens). The other half of the pigs were housed in barren pens $\left(\sim 1 \mathrm{~m}^{2} / \mathrm{pig}\right)$ with a partially slatted and partially concrete solid floor ( $n=40$ pens). Litter mates were equally distributed over barren and enriched pens. Two hands of wood shavings 
were given to barren-housed pigs each day from 6 weeks of age onwards. Each pen was cleaned daily and afterwards enriched-housed pigs received 3 $\mathrm{kg}$ of fresh wood shavings and fresh straw ( $250 \mathrm{~g}$ at the start of experiment and then gradually increasing to $1.5 \mathrm{~kg}$ ). A metal chain with a ball was present in each pen and at 8 weeks of age a jute sack was attached to the wall of each pen. The jute sack was replaced when worn.

Each pen contained six non-littermate pigs, three gilts and three barrows. Within each group, at least two pigs were classified as high-resister (HR) and two pigs as low-resister (LR) using a backtest (see below). Groups diverged in estimated breeding values for indirect genetic effects for growth during the finishing period (from app. 25 to $110 \mathrm{~kg}$ ), i.e. the heritable effect on the growth of their group members. During the finishing period, the growth of a pig is theoretically affected by each of its pen mates, and the genetic part of this effect is referred to as an indirect genetic effect for growth. All pigs in a pen had either an estimated relatively positive $(+\mid \mathrm{GE})$ or an estimated relatively negative indirect genetic effect on the growth of their pen mates (IGE), see (Camerlink et al. 2013; Reimert et al. 2014b) for details.

\subsubsection{Behavioural tests}

Backtest. Pigs were subjected to a backtest at approximately two weeks of age to assess their personality or coping style (Bolhuis et al. 2003). Briefly, a piglet was manually restrained in supine position for $60 \mathrm{~s}$. Classification of pigs was based on the number of struggles and the number of vocalizations. Piglets which showed two struggles and at least 25 vocalizations, or three struggles or more were classified as high-resisters (HR). The ones that 
showed 0 or 1 struggle or 2 struggles with less than 25 vocalizations were classified as low-resisters (LR).

Regrouping test. A regrouping test was conducted at 9 weeks of age as an acute stressor. Regrouping usually leads to fighting to establish a new rank order and is known to induce acute stress in pigs (Camerlink et al. 2013). In short, a pair of pigs was regrouped for $24 \mathrm{~h}$ in a new pen with two other pairs of unfamiliar pigs. The same housing condition and IGE class were maintained when mixing pairs of pigs, as well as a balanced group composition for gender and coping style. All pigs were returned to their original pen and group after the $24 \mathrm{~h}$ test.

\subsubsection{Blood collection and analyses}

Blood samples were taken before (week 8) and three days after the regrouping test (week 9), and at 22 weeks of age. Blood was collected by immobilizing a pig on its back in a crib (week 8 and week 9) or fixating the pig using a nose sling (week 22) after which blood was taken by venepuncture from the jugular vein. Housing condition and IGE class were taken into account in the order of blood collection. Blood was collected in serum separating tubes (Greiner bio-one, Kremsmünster, Austria) kept at room temperature (RT) until incubation for $1 \mathrm{~h}$ at $37^{\circ} \mathrm{C}$. After that samples were centrifuged at $5251 \mathrm{~g}$ for $12 \mathrm{~min}$ at $-20^{\circ} \mathrm{C}$. Sera were stored at $-80{ }^{\circ} \mathrm{C}$ until analysis.

\subsubsection{Enzyme-linked immunosorbent assay}

To be able to perform ELISA, IgG, IgM, and IgA antibody titers specific for phosphorylcholine were measured using PC conjugated to bovine serum 
albumin (PC-BSA, Santa Cruz Biotechnology, Santa Cruz, USA), and IgG and IgM antibody titers specific for myelin basic protein (MBP, Sigma-Aldrich, St. Louis, MO, USA) were determined by a two-step indirect enzyme-linked immunosorbent assay (ELISA) similar to Bolhuis et al. (2003). Preliminary analysis revealed too low IgA levels binding MBP for further analysis. First, medium binding microtiter plates (Greiner) were coated overnight at $4^{\circ} \mathrm{C}$ with $0.5 \mu \mathrm{g} / \mathrm{ml} \mathrm{PC-BSA}$ or $0.5 \mu \mathrm{g} / \mathrm{ml} \mathrm{MBP}$ in coating buffer $\left(0.05 \mathrm{M} \mathrm{Na}_{2} \mathrm{CO}_{3} \times\right.$ $10 \mathrm{H}_{2} \mathrm{O}, \mathrm{pH}$ 9.6). After washing with tap water containing $0.05 \%$ Tween 20 , serial dilutions of serum were added and incubated for 1.5 hour at RT. After washing, plates were incubated for 1.5 hour at RT with a 1: 20.000 (for MBP) and 1:40.000 (for PC-BSA) diluted peroxidase (PO)-conjugated goat antibody directed to swine $\operatorname{lgG}_{\mathrm{FC}}$ (GASwlgG $\mathrm{FC} / \mathrm{PO}$, Bethyl Laboratories, Montgomery, USA) to detect binding of IgG, or with 1: 20.000 (for MBP) and 1:40.000 (for PC-BSA) diluted PO-conjugated goat antibody directed to swine IgMFC (GASwlgM $\mathrm{FC}_{\mathrm{F}} / \mathrm{PO}$, Bethyl) to detect binding of IgM, or with 1:20.000 diluted PO-conjugated goat antibody directed to swine $\lg A_{F C}$ (GASwlgA $A_{F C} / P O$, Bethyl) to detect binding of IgA, respectively. After washing, tetramethylbenzidine (TMB) and $0.05 \% \mathrm{H}_{2} \mathrm{O}_{2}$ was added as a substrate and incubated for $10 \mathrm{~min}$ at RT. Reaction was stopped with $2.5 \mathrm{~N} \mathrm{H}_{2} \mathrm{SO}_{4}$ and absorbance was measured at $450 \mathrm{~nm}$ with a Multiskan (Flow, Irvine, UK). Each absorbance was expressed relatively to the absorbance of a standard positive control serum sample, and antibody titers were expressed as $\log _{2}$ values of dilutions that gave extinction closest to $50 \%$ of $E_{\max }$, where $E_{\max }$ represents the highest mean extinction of a standard positive serum present on every microtiter plate.

\subsubsection{Statistical analyses}


SAS (SAS 9.3, SAS Institute Inc.) was used for all statistical analyses. Titers could not be obtained from all 480 pigs at each sampling period due to technical problems, and due to pigs being removed from the experiment for health reasons (See Reimert et al., 2014b for details). From the enriched pigs, at 8,9 and 22 weeks of age, 236, 235, and 223 samples were analysed and from the barren pigs 237, 234 and 226 samples. PC-BSA-IgA titers were log transformed to obtain normality of residuals. Antibody titers were analysed using a repeated linear mixed model. The fixed effects housing, IGE class, backtest classification, week, and their interactions, and gender, and its interaction with week and batch were included in the model, as well as the random effect of pen and litter. Values in time of individual pens, pigs and litter were taken as repeated measurements, i.e. IGE class, housing and batch effects were tested against the random effect of pen, and backtest classification and gender effects were tested against the random effect of pig.

Preliminary analyses revealed strong week effects for all variables. Therefore, effects of housing, IGE class, backtest classification and gender were also analysed for each week separately. A linear mixed model was used for this, with housing, IGE class, backtest classification, their two-way interactions, gender, and batch as fixed effects and pen and litter as random effects. To investigate effects of housing, IGE class, backtest classification and gender on titers after acute stress, the delta between week 9 and week 8 was calculated and subsequently analysed with a linear mixed model with the same fixed, except week, and random effects as used for the per week analysis. Only significant effects $(p<0.05)$ and tendencies $(p<0.10)$ are reported. Significant interactions were further investigated with post hoc 
pairwise comparisons using the differences of the least square means. Results are presented as means \pm SEM.

\subsection{Results}

Titers of IgM, IgG and IgA antibodies in serum binding PC-BSA and IgM and IgG binding MBP are shown in Figure 2.1 (housing effects) and in Table 2.1 (gender effects). The analyses showed (additive, not interactive) effects of particularly housing and gender on titers over time, and therefore these two factors are highlighted separately. The change in antibody titers due to regrouping (delta week 9 - week 8 ) for both housing conditions is shown in Figure 2.2.

Table 2.1: Means and SEM of antibody titers of barrows and gilts determined before a $24 \mathrm{~h}$ regrouping test at 8 weeks of age, 3 days after the regrouping test at 9 weeks of age and at 22 weeks of age.

\begin{tabular}{|c|c|c|c|c|c|c|c|c|c|}
\hline & \multicolumn{3}{|c|}{ week 8} & \multicolumn{3}{|c|}{ week 9} & \multicolumn{3}{|c|}{ week 22} \\
\hline & barrow & gilt & $p$-value & barrow & gilt & $p$-value & barrow & gilt & $p$-value \\
\hline \multirow[t]{2}{*}{ PC-BSA-IgM } & $5.37 \pm 0.0$ & $5.42 \pm 0.0$ & ns & $6.56 \pm 0.0$ & $6.41 \pm 0.0$ & + & $7.98 \pm 0.1$ & $7.88 \pm 0.1$ & $*$ \\
\hline & 6 & 6 & & 8 & 8 & & 3 & 1 & \\
\hline \multirow[t]{2}{*}{ PC-BSA-IgG } & $5.64 \pm 0.1$ & $5.57 \pm 0.1$ & ns & $4.23 \pm 0.1$ & $4.34 \pm 0.1$ & ns & $2.78 \pm 0.1$ & $2.74 \pm 0.1$ & ns \\
\hline & 2 & 3 & & 3 & 3 & & 3 & 3 & \\
\hline \multirow[t]{2}{*}{ PC-BSA-IgA } & $0.86 \pm 0.0$ & $0.75 \pm 0.0$ & + & $0.81 \pm 0.0$ & $0.92 \pm 0.0$ & ns & $1.20 \pm 0.0$ & $1.02 \pm 0.0$ & $*$ \\
\hline & 6 & 6 & & 5 & 7 & & 5 & 6 & \\
\hline \multirow[t]{2}{*}{ MBP-IgM } & $5.80 \pm 0.0$ & $5.69 \pm 0.0$ & ns & $5.74 \pm 0.0$ & $5.75 \pm 0.0$ & ns & $7.24 \pm 0.0$ & $7.15 \pm 0.0$ & + \\
\hline & 9 & 9 & & 7 & 7 & & 6 & 6 & \\
\hline \multirow[t]{2}{*}{ MBP-IgG } & $4.40 \pm 0.0$ & $4.28 \pm 0.0$ & ns & $4.63 \pm 0.1$ & $4.35 \pm 0.0$ & $*$ & $5.25 \pm 0.1$ & $5.18 \pm 0.1$ & ns \\
\hline & 8 & 8 & & 0 & 9 & & 0 & 0 & \\
\hline
\end{tabular}

Effects of gender over all three weeks are reported in the text; significances of gender differences per week are indicated: ${ }^{*} p<0.05 ;{ }^{+} p<0.10$, and $n$ s for non-significant. 


\subsubsection{IgM, IgG and IgA titers binding PC-BSA}

IgM binding PC-BSA. Titers of IgM binding PC-BSA tended to be affected by gender $(p<0.10)$, with overall lower levels for gilts $(6.56 \pm 0.06)$ than for barrows $(6.60 \pm 0.07$, see also Table 2.1$)$ and were also affected by week $(p<0.001)$. Post hoc analysis showed that titers increased from week 8 to 9 to 22 (Figure 2.1A). When analysing the effects per week, barren-housed pigs had higher IgM titers binding PC-BSA than enriched-housed pigs in week 8 $(p<0.05)$, and they tended to do so in week $9(p<0.10$, Figure 2.1A). The increase in PC-BSA-IgM titers after regrouping (i.e. delta of week 9 minus week 8 as an effect of regrouping) was larger for barrows $(1.17 \pm 0.08)$ than for gilts $(0.99 \pm 0.07, \mathrm{P}<0.05)$. There was no effect of housing on titer changes after regrouping (Figure 2.2).

IgG binding PC-BSA. PC-BSA-IgG titers were affected by week $(\mathrm{p}<0.001)$ and decreased from week 8 to 9 to 22 (Figure 2.1B). When analysed for each week separately, barren-housed pigs had lower titers than enriched-housed pigs in week $9(p<0.05)$ and in week $22(p<0.10)$, with no housing effect in week 8 (Figure 2.1B). Gender did not affect PC-BSA-IgG titers (see Table 2.1 for means per week). The change in PC-BSA-IgG titers after regrouping (i.e. delta) was not affected by any of the factors tested (Figure 2.2).

IgA binding PC-BSA. PC-BSA-IgA titers tended to be affected by housing $(p<0.10)$, with lower levels for barren-housed pigs than for enriched-housed pigs (overall: $0.99 \pm 0.04$ vs. $0.87 \pm 0.04$, Figure $2.1 C$ ), particularly in week 9 and 22 (per week analysis, both $\mathrm{P}<0.001)$. In addition, effects of gender $(p<0.05)$, week $(p<0.001)$, and the interaction between gender and week $(p<0.05)$ were found. Post hoc analysis showed that overall titers in week 8 and week 
9 were not significantly different, but differed from those in week 22. In gilts, however, PC-BSA-IgA titers in week 8 differed from those in week $9(p<0.05$, see Table 2.1 for means). Barrows had higher titers than gilts in week 22 (Table 2.1). The change in titers from week 8 to week 9 (i.e. delta) tended to be affected by housing (See Figure $2.2, p<0.10$ ) and was affected by gender (gilts: $0.17 \pm 0.08$; barrows: $-0.06 \pm 0.07, p<0.05$ ).

\subsubsection{IgM and IgG titers binding MBP}

IgM binding MBP. MBP-IgM titers were affected by housing $(\mathrm{p}<0.001)$ (Figure 2.1D), with overall lower titers for barren-housed pigs $(6.10 \pm 0.10)$ than enriched-housed pigs $(6.36 \pm 0.10)$. Separate analyses per week revealed that MBP-IgM titers were particularly affected by housing in week $9(p<0.05)$ and week $22(p<0.05)$ (Figure 2.1D). Also, a tendency for a gender effect was found $(p<0.10)$, with overall lower titers for gilts $(6.19 \pm 0.05)$ than for barrows (6.24 \pm 0.05$)$. MBP-IgM titers were higher in week $22(7.20 \pm 0.04)$ compared to week $8(5.75 \pm 0.05)$ and week $9(5.75 \pm 0.05$, week effect, $p<0.05)$. Even though titers in week 8 and 9 were overall not significantly different, gender $(p<0.10)$ tended to affect the delta in MBP-IgM titers (gilts: $0.10 \pm 0.08$; barrows: $-0.10 \pm 0.08)$.

IgG binding MBP. MBP-IgG titers tended to be affected by housing $(\mathrm{p}<0.10)$ (Figure 2.1E), and were affected by gender $(p<0.05)$ and week $(p<0.001$, Figure 2.1E). Barren-housed pigs $(4.60 \pm 0.09)$ tended to have lower titers than enriched-housed pigs $(4.78 \pm 0.10)$. Analysis of the effects for each week separately showed that MBP-IgG titers particularly tended to be affected by housing $(p<0.10)$ in week 9. Gilts $(4.60 \pm 0.05)$ had lower titers than barrows (4.75 \pm 0.06$)$. Post hoc analysis showed those titers in week 22 were higher 
than those in week 8 and week 9. The change in MBP-lgG titers from week 8 to week 9 tended to be influenced by gender $(p<0.10$; delta gilts: $0.05 \pm 0.08$; barrows: $0.28 \pm 0.10$ ).

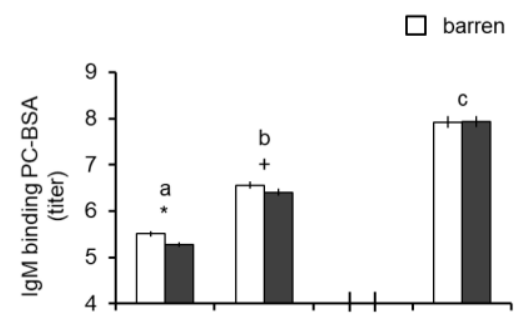

$\square$ enriched
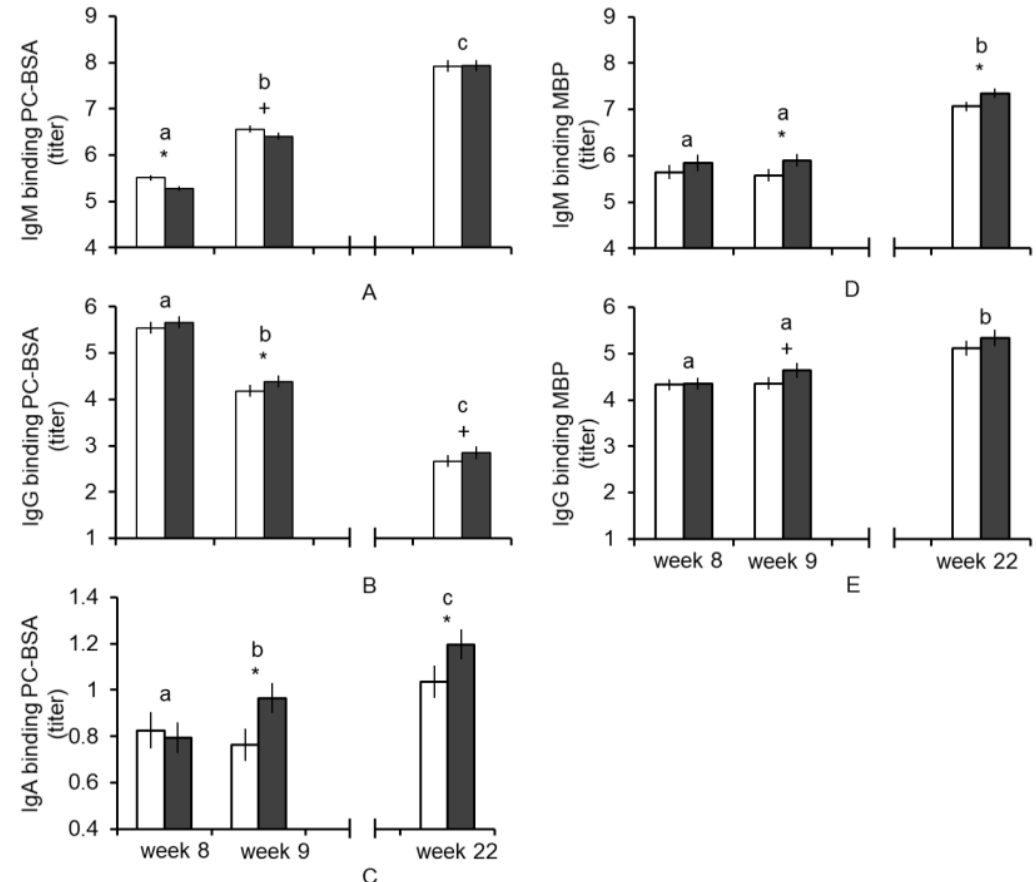

Figure 2.1: Means and SEM of antibody titers. $\lg M(A), \lg G(B)$ and $\lg A(C)$ titers to phosphorylcholine-bovine serum albumin (PC-BSA) and IgM (D) and IgG (E) titers to myelin basic protein (MBP) of pigs ( $n=80$ pens) in barren and enriched housing conditions measured before a $24 \mathrm{~h}$ regrouping test at 8 weeks of age, 3 days after the regrouping test at 9 weeks of age and at 22 weeks of age. Effects of housing over all three weeks are reported in the text; significances of housing differences per week are indicated: ${ }^{*} p<0.05 ;{ }^{+} p<0.10$. Week differences are indicated by letters; weeks lacking a common letter significantly differ. 


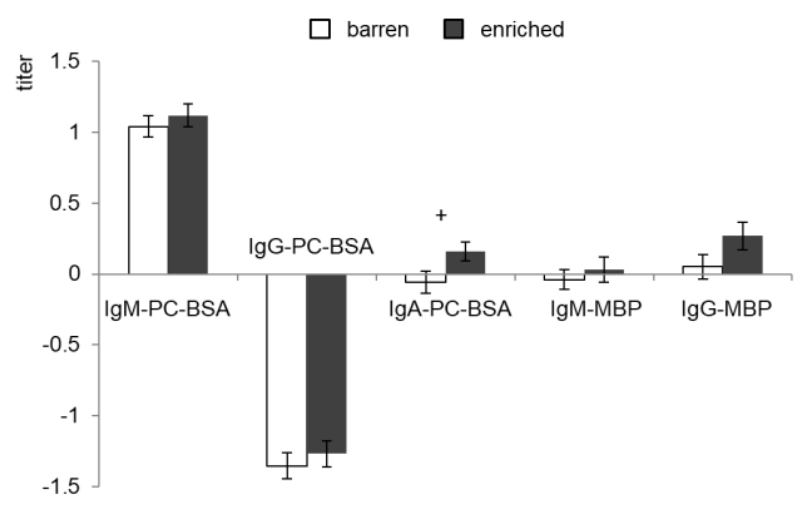

Figure 2.2: Means and SEM of the variation in antibody titers between week 9, 3 days after a regrouping test, and week 8, before this test. IgM, IgG and IgA titers to phosphorylcholine-bovine serum albumin (PC-BSA) and IgM and IgG titers to myelin basic protein (MBP) of pigs ( $n=80$ pens) in barren and enriched housing conditions for delta (week 9 - week 8). Significances of housing differences are indicated: ${ }^{+} p<0.10$.

\subsubsection{Effect of litter and individual characteristics}

All auto-antibody titers were affected by litter $(p<0.001)$. Litter did not affect the titer changes from week 8 to week 9 (after the regrouping test), with the exception of changes in PC-BSA-IgG titers $(p<0.05)$. Backtest classification or IGE class did not affect antibody titers, except that PC-BSA-IgM tended $(p<0.10)$ to be higher for -IGE pigs $(6.59 \pm 0.08)$ than for +IGE pigs $(6.38 \pm 0.08)$ in week 9. 


\subsection{Discussion}

In this study, we investigated the chronic effects of a straw-enriched vs. a barren housing environment, and the acute effects of regrouping on levels of NAAb binding PC-BSA and MBP in pigs differing in gender, coping style and genetic background. Our study shows that these antibodies can be detected in blood of healthy pigs, and that their levels are related to housing conditions, regrouping stress and gender.

\subsubsection{Housing effects}

In the present study, serum levels of auto-antibodies binding MBP were higher in pigs kept in an enriched environment from weaning at 4 weeks onward as compared to barren-housed pigs. As we distributed pigs from the same litters in a balanced manner over the two different post-weaning housing conditions and studied 40 pens per housing condition ( 80 pens in total), it is highly unlikely that these housing differences found in week 8 (and 9 and 22) are an artefact of coincidental differences that might have already existed in week 4. The housing effect on MBP titers was strongest for the IgM isotype. In man, NAAb (including MBP antibodies) in cerebrospinal fluid and blood have frequently been associated with neurologic diseases (Gold et al. 2012). The sera of healthy humans, however, also contain MBP autoantibodies (Hedegaard et al. 2009). Auto-antibodies binding neural antigens may both have a pathological role in neural tissue, and a protective role by regulating immune homeostasis (Gold et al. 2012). The latter may hold for our healthy pigs as antibody titers to MBP were higher in enriched-housed pigs. Other studies have shown an impact of enrichment on other immune characteristics of pigs (Bolhuis et al. 2003; Reimert et al. 2014b), including 
reduced haptoglobin levels (Reimert et al. 2014b), and provided evidence that pigs reared in an enriched environment were less susceptible to a porcine reproductive and respiratory virus (PRRSV) combined with actinobacillus pleuropneumoniae (APP) challenge (van Dixhoorn et al. 2016).

We can only speculate about the origin of the effect of enrichment on levels of MBP-binding auto-antibodies of the IgM isotype. First, the higher antibody levels may be related to the effects of enrichment on brain and behaviour of pigs, including their mental state. In humans, it has been suggested that emotional states, for instance anxiety and depression, could trigger immune alterations (Postal and Appenzeller 2015), which may, in turn, affect (neural) cell injury, and as a consequence the release of cell constituents such as MBP (Abou-Donia et al. 2013). Second, a difference in hygienic conditions between the enriched and barren environment may have played a role, even though all pigs were housed in the same room. In this study, enrichment was provided in the form of straw bedding which is beneficial for pigs' behaviour and welfare (Camerlink et al. 2015), but can also be a suitable environment for microbes (Tuyttens 2005) and in this manner affect immune competence. Alternatively, lower MBP-binding antibody titers in barren housed pigs may reflect a continuous usage of the antibodies due to enhanced formation of immune complexes with MBP. Further research is needed to disentangle these potential effects of enrichment on the immune state of pigs, including their auto-antibody levels.

Enrichment as compared with barren housing led to lower IgM and higher IgG antibodies binding PC-BSA at some time points when we analysed levels of antibodies per week, and tended to increase overall titers of IgA binding 
PC-BSA. The response of PC-BSA to regrouping (see below) was generally not affected by housing, except for a tendency of enriched-housed pigs to show a higher increase in IgA-PC-BSA antibodies after regrouping. Apart from the potential influencing factors as discussed above for MBP, a housing difference in aggressive behaviour of these pigs over time, causing body lesions and thus cell damage (Camerlink et al. 2013) may explain the effects of enrichment on PC-BSA auto-antibody levels. The exact consequences of these subtle differences in auto-antibody levels in our study are unknown, but previous studies have found (even modest differences in) NAb to be linked with survival (Sun et al. 2011) and fitness (Rossi et al. 2013).

In brief, effects of housing on MBP auto-antibody (mainly IgM) titers and to a lesser extent on PC antibody titers tentatively suggest that environmental conditions affect levels of auto-antibodies binding (neural) antigens as a homeostatic response, which could have consequences for both pig health and welfare.

\subsubsection{Regrouping effects}

Remarkably, IgM and IgG binding PC-BSA, titers were significantly different between week 8 and week 9 , three days after the regrouping test. A similar effect was found for the titers of IgA binding PC-BSA, but in gilts only. An age effect seems unlikely here, given the short time span between both sampling days, suggesting an effect of acute stress. A previous study on the same pigs revealed that temporary regrouping activated the classical complement pathway and increased serum haptoglobin levels, suggesting acute regrouping stress (Reimert et al. 2014b). Also others reported a rise in serum auto-antibodies after acute (restraint) stress (Andrejević et al. 1997). 
Regrouping leads to mutual ranking fights, which can be quite vigorous. It is known to be stressful for pigs and the aggressive behaviour results in body lesions and scratches, in this regrouping test on average more than 70 per animal (Camerlink et al. 2013). The cell damage associated with stress and/or body lesions induced release of PC could explain why merely NAAb binding PC-BSA, but not those binding MBP, was affected by regrouping.

\subsubsection{Week effects}

All antibody titers measured were affected by week, and were generally highest at 22 weeks of age, except PC-BSA-IgG. The increase in antibody titers with time is in line with other studies reporting a rise of natural antibody titers with age (Reimert et al. 2014b). In contrast, the level of IgG binding PCBSA was lower in week 22 compared to week 8 and week 9. A Spearman correlation test showed that PC-BSA-IgM and PC-BSA-IgG titers were significantly negatively correlated. Kim et al. (2002) tested the idea that IgM is responsible for complement activation on apoptotic cells via binding of antibodies targeting lysophospholipids, including PC. Under normal circumstances, lysophospholipids are recognized by IgM which activates the classical complement pathway preventing inflammation. In this study, pigs were all healthy and environmental enrichment or barren conditions may not have led to a shortage of IgM antibodies. When enough IgM is available, the animals can handle the clearance of PC that has been formed as a waste product due to stress. If however, there is not enough IgM, IgG may be formed which might explain why there was a negative correlation between levels of PC-BSA-IgM and levels of PC-BSA-IgG.

\subsubsection{Gender effects}


NAAb can also be affected by gender (Nagele et al. 2013). Except for IgG titers binding PC-BSA, all antibody titers measured in this study were affected or tended to be affected by gender. In general, barrows showed higher antibody titers binding PC-BSA and MBP compared to gilts. Nagele et al. (2013) also found higher NAAb levels in human males which would be in line with our study, although it should be noted that the male pigs were castrated. In general, the immune system of males and females differs significantly (Bupp 2015).

Changes in antibody titers from week 8 to week 9 after regrouping were also affected by gender, with the most marked effect on IgA titers which clearly increased in gilts, but decreased in barrows. In week 9, IgA titers binding PCBSA in gilts were higher than in barrows, which may show a difference in how gilts and barrows deal immunologically with stress after regrouping. Whether sex steroids could underlie the immunological differences found between gilts and barrows remains to be studied. However, gilts and barrows, including the animals in this study, have been found to behave very differently in various novelty tests; e.g. (Reimert et al. 2014a) and in regrouping tests (Camerlink et al. 2013). Differences in dealing both behaviourally and immunologically with stress may partly explain the different auto-antibody responses to regrouping in gilts and barrows.

\subsubsection{Coping style and indirect genetic effects}

Previous studies have shown that pigs diverging in coping style, i.e. the way they deal with stress as part of their personality, differ in immune characteristics, including natural antibodies to $\mathrm{KLH}$, and/or modulate the effects of environmental factors on immune responses (Bolhuis et al. 2003; 
Oster et al. 2014; Reimert et al. 2014b; Schrama et al. 1997). No effects on levels of auto-antibodies binding MBP and PC were, however, found in this study. Classification of pigs based on their indirect genetic effect on the growth of their pen mates, which is known to influence fear levels (Reimert et al. 2014a), aggression (Camerlink et al. 2013), occurrence of abnormal behaviour (Camerlink et al. 2015), and some (other) immune characteristics (Reimert et al. 2014b) generally did not affect titers of auto-antibodies binding MBP and PC either, except that PC-BSA-IgM tended to be higher for -IGE pigs than for +IGE pigs in week 9. The latter could be related to the higher level of aggression found in -IGE pigs when reunited with their own pen mates after the regrouping test (Camerlink et al. 2013).

\subsubsection{Litter effects}

Litter strongly affected titers of NAAb binding MBP and PC, in spite of the fact that at weaning, litters were split up, with siblings being equally distributed to barren vs. enriched pens. The effect of litter on these antibodies could partly be related to the shared prenatal and early postnatal environment (until weaning at 4 weeks of age) of the sibling piglets, but could also reflect genetic effects. A genetic basis for natural antibodies has been demonstrated in other species, such as cattle (Wijga et al. 2013), and poultry (Bao et al. 2016). To the best of our knowledge, it is unknown whether natural autoantibody levels and their response to acute challenges are heritable in pigs. 


\subsection{Conclusion}

In conclusion, environment, gender, litter and possibly regrouping stress affected blood levels of auto-antibodies related with neural antigen (MBP) and cell damage (PC). In this study, enriched-housed healthy pigs were found to have higher levels of IgM and IgG binding MBP, and IgA binding PC-BSA in serum than barren-housed pigs which may reflect or affect their immune competence, (mental) health and welfare. More research is warranted to investigate the mechanisms by which environmental enrichment affects NAAb.

\subsection{Implications}

Since January 2003, the provision of appropriate environmental enrichment to pigs has been mandatory across the European Union (EU) (Directives 2001/88/EC and 2001/93/EC). Pigs may experience stress and develop abnormal behaviour due to barren housing conditions in commercial pig husbandry and standard management procedures. This might affect their immune status, and reduce welfare. The present paper suggests that enriched housing influenced the levels of natural auto-antibodies binding danger and neural antigens, which may reflect or affect their immune competence, (mental) health and welfare. 


\subsection{Acknowledgements}

The authors would like to thank Mike Nieuwland, Ger de Vries Reilingh, Monique Ooms, Fleur Bartels, Irene Camerlink, Merel Verhoeven, Nanda Ursinus and Bjorge Laurenssen for skillful assistance in conducting the experiment and analyses. We are also grateful to the animal caretakers and students involved. Lu Luo is financed by the China Scholarship Council. 


\subsection{References}

Abou-Donia MB, Abou-Donia MM, EIMasry EM, Monro JA, Mulder MF (2013) Autoantibodies to nervous system-specific proteins are elevated in sera of flight crew members: biomarkers for nervous system injury Journal of Toxicology and Environmental Health, Part A 76:363-380

Andrejević $S$ et al. (1997) Stress-induced rise in serum anti-brain autoantibody levels in the rat International journal of neuroscience 89:153-164

Bao M, Bovenhuis H, Nieuwland M, Parmentier H, van der Poel J (2016) Genetic parameters of $\operatorname{lgM}$ and $\lg G$ antibodies binding autoantigens in healthy chickens Poultry science 95:458-465

Bolhuis JE, Parmentier HK, Schouten WG, Schrama JW, Wiegant VM (2003) Effects of housing and individual coping characteristics on immune responses of pigs Physiology \& behavior 79:289-296

Bolhuis JE, Schouten WG, Schrama JW, Wiegant VM (2005) Behavioural development of pigs with different coping characteristics in barren and substrate-enriched housing conditions Applied Animal Behaviour Science 93:213-228

Bupp MRG (2015) Sex, the aging immune system, and chronic disease Cellular immunology 294:102-110

Camerlink I, Bolhuis J, Duijvesteijn N, Van Arendonk J, Bijma P (2014) Growth performance and carcass traits in pigs selected for indirect genetic effects on growth rate in two environments Journal of Animal Science 92:2612-2619

Camerlink I, Turner SP, Bijma P, Bolhuis JE (2013) Indirect genetic effects and housing conditions in relation to aggressive behaviour in pigs PloS one 8:e65136

Camerlink I, Ursinus WW, Bijma P, Kemp B, Bolhuis JE (2015) Indirect genetic effects for growth rate in domestic pigs alter aggressive and manipulative biting behaviour Behavior genetics 45:117-126

Casali P, Notkins AL (1989) Probing the human B-cell repertoire with EBV: polyreactive antibodies and CD5+ B lymphocytes Annual review of immunology 7:513-535

de Jong IC, Prelle IT, van de Burgwal JA, Lambooij E, Korte SM, Blokhuis HJ, Koolhaas JM (2000) Effects of environmental enrichment on behavioral responses to novelty, learning, and memory, and the circadian rhythm in cortisol in growing pigs Physiology \& behavior 68:571-578 
Dhabhar FS (2014) Effects of stress on immune function: the good, the bad, and the beautiful Immunologic research 58:193-210

Douglas C, Bateson M, Walsh C, Bédué A, Edwards SA (2012) Environmental enrichment induces optimistic cognitive biases in pigs Applied Animal Behaviour Science 139:65-73

Gold M, Pul R, Bach JP, Stangel M, Dodel R (2012) Pathogenic and physiological autoantibodies in the central nervous system Immunological reviews 248:68-86

Grimberg-Henrici CG, Vermaak P, Bolhuis JE, Nordquist RE, van der Staay FJ (2016) Effects of environmental enrichment on cognitive performance of pigs in a spatial holeboard discrimination task Animal cognition 19:271-283

Hedegaard CJ, Chen N, Sellebjerg F, Sørensen PS, Leslie RGQ, Bendtzen K, Nielsen CH (2009) Autoantibodies to myelin basic protein (MBP) in healthy individuals and in patients with multiple sclerosis: a role in regulating cytokine responses to MBP Immunology 128:e451-e461

Kim SJ, Gershov D, Ma X, Brot N, Elkon KB (2002) I-PLA2 activation during apoptosis promotes the exposure of membrane lysophosphatidylcholine leading to binding by natural immunoglobulin $\mathrm{M}$ antibodies and complement activation The Journal of experimental medicine 196:655-665

Koh KB (1998) Emotion and immunity Journal of Psychosomatic Research 45:107-115

Lutz HU, Binder CJ, Kaveri S (2009) Naturally occurring auto-antibodies in homeostasis and disease Trends in immunology 30:43-51

Maier SF, Watkins LR (1998) Cytokines for psychologists: implications of bidirectional immune-to-brain communication for understanding behavior, mood, and cognition Psychological review 105:83

Mayasari N, de Vries Reilingh G, Nieuwland M, Remmelink G, Parmentier H, Kemp B, van Knegsel A (2015) Effect of maternal dry period length on colostrum immunoglobulin content and on natural and specific antibody titers in calves Journal of dairy science 98:3969-3979

Nagele EP, Han M, Acharya NK, DeMarshall C, Kosciuk MC, Nagele RG (2013) Natural IgG autoantibodies are abundant and ubiquitous in human sera, and their number is influenced by age, gender, and disease PLoS One 8:e60726

Oster M et al. (2014) Transcriptional responses of PBMC in psychosocially stressed animals indicate an alerting of the immune system in female but not in castrated male pigs BMC genomics 15:967 
Panda S, Ding JL (2015) Natural antibodies bridge innate and adaptive immunity The journal of immunology 194:13-20

Postal M, Appenzeller S (2015) The importance of cytokines and autoantibodies in depression Autoimmunity reviews 14:30-35

Reimert I, Rodenburg TB, Ursinus WW, Kemp B, Bolhuis JE (2014a) Responses to novel situations of female and castrated male pigs with divergent social breeding values and different backtest classifications in barren and straw-enriched housing Applied animal behaviour science 151:24-35

Reimert I, Rodenburg TB, Ursinus WW, Kemp B, Bolhuis JE (2014b) Selection based on indirect genetic effects for growth, environmental enrichment and coping style affect the immune status of pigs PloS one 9:e108700

Rossi S, Doucelin A, Le Potier M-F, Eraud C, Gilot-Fromont E (2013) Innate immunity correlates with host fitness in wild boar (Sus scrofa) exposed to classical swine fever PLoS One 8:e79706

Scholz J, Allemang-Grand R, Dazai J, Lerch JP (2015) Environmental enrichment is associated with rapid volumetric brain changes in adult mice Neurolmage 109:190-198

Schrama J, Schouten J, Swinkels J, Gentry J, de Vries Reilingh G, Parmentier H (1997) Effect of hemoglobin status on humoral immune response of weanling pigs differing in coping styles Journal of animal science 75:2588-2596

Sun Y, Parmentier H, Frankena K, Van der Poel J (2011) Natural antibody isotypes as predictors of survival in laying hens Poultry science 90:2263-2274

Tuyttens FAM (2005) The importance of straw for pig and cattle welfare: a review Applied animal behaviour science 92:261-282

van Dixhoorn ID et al. (2016) Enriched housing reduces disease susceptibility to co-infection with porcine reproductive and respiratory virus (PRRSV) and Actinobacillus pleuropneumoniae (A. pleuropneumoniae) in young pigs PloS one 11:e0161832

Wijga S, Bovenhuis H, Bastiaansen J, Arendonk J, Ploegaert T, Tijhaar E, Poel $J$ (2013) Genetic parameters for natural antibody isotype titers in milk of Dutch Holstein-Friesians Animal genetics 44:485-492

Zhou Y, Cheshire A, Howell LA, Ryan DH, Harris RB (1999) Neuroautoantibody immunoreactivity in relation to aging and stress in apolipoprotein $\mathrm{E}$ deficient mice Brain research bulletin 49:173-179 



\section{Chapter 3}

\section{Effect of enriched housing on levels of natural (auto-) antibodies in pigs co-infected with porcine reproductive and respiratory syndrome virus (PRRSV) and Actinobacillus pleuropneumoniae}

L. Luo ${ }^{1}$, I. D. E van Dixhoorn ${ }^{2}$, I. Reimert ${ }^{1}$, B. Kemp ${ }^{1}$, J. E. Bolhuis ${ }^{1}$, H. K. Parmentier $^{1 *}$

${ }^{1}$ Adaptation Physiology Group, Department of Animal Sciences, Wageningen University \& Research, P.O. Box 338, 6700 AH, The Netherlands

${ }^{2}$ Wageningen Livestock Research, P.O. Box 338, $6700 \mathrm{AH}$, The Netherlands

${ }^{*}$ Corresponding author: H. K. Parmentier

E-mail address: henk.parmentier@wur.nl 


\subsection{Abstract}

Housing of pigs in barren, stimulus-poor housing conditions may influence their immune status, including antibody responses to (auto-)antigens, and thus affect immune protection, which will influence the onset and outcome of infection. In the present study, we investigated the effects of environmental enrichment versus barren housing on the level of natural (auto-)antibodies (NA(A)b) and their isotypes (IgM and $\operatorname{IgG}$ ) binding keyhole limpet hemocyanin (KLH), myelin basic protein (MBP), and phosphorycholine conjugated to bovine serum albumin (PC-BSA) in pigs co-infected with porcine reproductive and respiratory syndrome virus (PRRSV) and Actinobacillus pleuropneumoniae (A. pleuropneumoniae). Pigs $(n=56)$ were housed in either barren or enriched pens from birth to 54 days of age. They were infected with PRRSV on 44 days of age, and with A. pleuropneumoniae 8 days later. Blood samples were taken on 7 different sampling days. Housing significantly affected the overall serum levels of $N A(A) b$ binding $K L H, M B P$ and PC-BSA, and before infection barren housed pigs had significantly higher levels of $N A(A) b$ than enriched housed pigs, except for KLH-IgM and PC-BSAIgG. Infection only affected the IgM, but not the IgG isotype. Moreover, changes in MBP-IgM and PC-BSA-IgM following infection were different for enriched and barren housed pigs. These results suggest that the effect of infection on $N A(A) b$ is influenced by housing conditions and that $N A(A) b$, especially IgM may be affected by infection. 


\subsection{Introduction}

Commercial pigs usually live in barren, stimulus-poor housing conditions. The limited possibilities to express important behaviours under such conditions may lead to signs of chronic stress (Bolhuis et al. 2006; Carreras et al. 2016; de Jong et al. 2000), and the development of damaging behaviours directed at group members, such as tail or ear biting (Bolhuis et al. 2005; Scott et al. 2009; Telkänranta et al. 2014). It has been suggested that housing, stress and social factors are associated with disease susceptibility and therefore environmental conditions have been indicated as cofactors in the pathogenesis of infectious diseases (Proudfoot and Habing 2015; van Dixhoorn et al. 2016). Several studies reported immunological differences between pigs kept in barren conditions as compared to pigs provided with environmental enrichment, such as substrates to manipulate, or extra space (de Groot et al. 2000; Ernst et al. 2006; Merlot et al. 2012). Moreover, a recent study (van Dixhoorn et al. 2016) reported that pigs kept in enriched pens, showed a faster viral clearance and were less likely to develop lung lesions following a porcine reproductive and respiratory syndrome virus (PRRSV) and Actinobacillus pleuropneumoniae (A. pleuropneumoniae) coinfection as compared to barren housed pigs. PRRSV and $A$. pleuropneumoniae are pathogens that are frequently involved in the porcine respiratory disease complex (PRDC), which causes severe health and welfare problems, and major economic losses in swine industry (Lévesque et al. 2014).

We recently demonstrated that enriched housed pigs had higher levels of natural autoantibodies binding danger (phosphorycholine, PC) and neural 
(myelin basic protein, MBP) auto-antigens than barren housed pigs (Luo et al. 2017). PC is a component of cell membranes and levels of anti-PC antibodies may reflect cell damage and inflammation related to stress (Lutz et al. 2009). MBP is an important protein in the nervous system, and levels of antibodies binding MBP were affected by mental stress (Abou-Donia et al. 2013). Furthermore, Reimert et al. (Reimert et al. 2014) reported that enriched housed pigs had higher levels of natural antibodies (NAb) to keyhole limpet hemocyanin (KLH) than barren housed pigs. KLH is an antigen without known cross reactivity with mammalian antigens and it has been widely used to detect natural antibodies in a variety of species, including pigs (Reimert et al. 2014). NAb appear in the absence of apparent antigenic stimulation and are secreted by long lived, self-renewing B1 cells. NAb have been proposed to be an essential part of the first line of defence against viral and bacterial infections (Diamond et al. 2003; Gao et al. 2012; Lobo 2016). A large part of NAb (70-80\%) binds to auto-antigens. Due to low affinity and poly-specificity, both natural IgM and natural IgG may protect the host by binding to bacteria and viruses by enhancing phagocytosis. IgM antibodies play a role in clearing apoptotic cells, maintain B cell homeostasis (Quartier et al. 2005), and can simultaneously bind to different conserved structures, like PC, nucleic acids, and carbohydrate on the same pathogen and inhibit the pathogen from invading cells and dispersing into different organs (Ehrenstein and Notley 2010; Lobo 2016). Natural IgM may thus limit the spread of infection (Lobo et al. 2008a; Ochsenbein et al. 1999), and as such provide defence against viral infections directly by inhibiting spread of virus or by T cell activation or by binding with viral and chemokine receptors (Lobo et al. 2008a). IgG can recognize a range of Gram-negative and Gram-positive bacteria with the aid 
of serum lectin pattern recognition receptors (PRRs) which are known to bind to sugar residues on the bacteria. In this way, IgG can collaborate with lectin, thus swiftly and effectively kill invading pathogens (Panda et al. 2013). It has been proposed that natural IgM-producing $B$ cells do not undergo affinity maturation or activation, and subsequently shift to $\operatorname{lgG}$, but in contrast to antigen-specific immunoglobulin producing B2 cells, provide a readily available poly-specific source of defence, in response to activation of innate receptors, like TLRs (Lobo 2016).

Studies reported that levels of $\mathrm{NA}(\mathrm{A}) \mathrm{b}$ may rise with age but are independent of the (antigenic) environment. However, in a former study, effects of housing conditions on levels of NA(A)b were found (Luo et al. 2017). In the current study we studied the effect of housing and a co-infection with PRRSV and $A$. pleuropneumoniae on levels of natural IgM and IgG binding KLH, MBP and PC-BSA. To that aim, we studied first, before infection, the effects of housing on these $N A(A) b$ and their changes in time, and second, after infection, the interactive effect of infection and housing on NA(A)b changes.

\subsection{Materials and methods}

The established principles of laboratory animal use and the EU and Dutch laws related to animal experiments were adhered to in this study.

\subsubsection{Animals and housing}

We studied serum samples from 56 male and female piglets (Topigs 20 line, from Great Yorkshire $\times$ Landrace origin), the same pigs as described in (van Dixhoorn et al. 2016). In short, piglets were offspring of 8 multiparous sows 
obtained from a PRRSV and A. pleuropneumoniae-free herd. Sows were inseminated on the same day and the expected parturition day was defined as day 0 for all piglets. Sows were housed in farrowing crates at research facility A of Wageningen University Livestock Research, the Netherlands from two weeks before parturition. From the first day of life onwards, half of the pigs were housed in conventional $5 \mathrm{~m}^{2}$ barren pens with $100 \%$ slatted floor and a $100 \times 45 \mathrm{~cm}$ solid rubber floor mat. The other half of the pigs were housed in $10 \mathrm{~m}^{2}$ enriched pens with partly slatted (40\%) and partly solid (60\%) floors. In both the enriched and barren pens, two chains with blocks were added as enrichment. Two jute bags and branches of a broom were provided in the enriched pens, and they were replaced weekly. Additionally, $1 \mathrm{~kg}$ straw, $160 \mathrm{~L}$ of moist peat and $180 \mathrm{~L}$ of wood shavings were provided in the enriched pens as rooting substrate. Straw and wood shavings were replenished daily ( $0.5 \mathrm{~kg} /$ day straw, $23 \mathrm{~L} /$ day of wood shavings), and $20 \mathrm{~L}$ of fresh peat was added weekly. From 13 days of age until weaning, the panels between two adjacent enriched pens were removed. Thus, the four individual enriched pens of $10 \mathrm{~m}^{2}$ were temporarily transformed into two pens of $20 \mathrm{~m}^{2}$ to enable early social interaction between two enrichedhoused litters. Each pen was cleaned daily and enrichment materials and food were $\gamma$-irradiated (9kGy irradiation; Synergy Health Ede BV, the Netherlands). During the first week after birth, a heating lamp was provided in each pen. Each pen had two drinking nozzles, one for the sow and one for the piglets. Sows were fed a standard commercial diet twice a day. From 3 days of age, the piglets received solid food ad libitum. Lights in the pens were on between $6: 00$ am and $6: 00 \mathrm{pm}$, and the temperature was $25^{\circ} \mathrm{C}$, and gradually decreased to $22{ }^{\circ} \mathrm{C}$ the week before weaning. 


\subsubsection{Weaning, regrouping and relocation}

Piglets were selected at weaning (31 days of age). Seven piglets per litter were selected to regroup into 8 new experimental groups. All piglets were equally mixed, meaning an equal number of unfamiliar pigs per group and new groups with comparable compositions were formed. Gender, bodyweight, and coping style (see (van Dixhoorn et al. 2016)) were taken into account in the selection of piglets, and housing treatment (barren vs. enriched) for each piglet was kept the same as before weaning.

At 39 days of age, all piglets were transported to facility $B$ of Wageningen University Livestock Research, the Netherlands. Pigs from 2 barren and 2 enriched groups were housed in separate High Efficiency Particulate Air (HEPA) filtered animal rooms, and the other 2 barren and 2 enriched groups were kept in separate pens within one large room without extra biosafety measures as negative-controls. Housing conditions, group structure, pen sizes and access to food and water remained the same as before transport.

On the day of weaning, the temperature was $28^{\circ} \mathrm{C}$ and was decreased by $2{ }^{\circ} \mathrm{C}$ each week until it reached $22{ }^{\circ} \mathrm{C}$. It was kept at $22{ }^{\circ} \mathrm{C}$ until the end of the experiment.

\subsubsection{Infection procedure}

At 44 days of age, half of barren and enriched housed pigs were inoculated intra-nasally with $1.5 \mathrm{~mL}$ inoculum containing $5 \log _{10} 50 \%$ tissue culture infectious dose $\left(\mathrm{TCID}_{50}\right)$ of the mild-virulent European PRRSV serotype I strain LV-Ter Huurne. At 52 days of age, pigs were infected with $A$. 
pleuropneumoniae by aerosol. See for more details (van Dixhoorn et al. 2016).

Half of the barren control and enriched control pigs were not inoculated (negative controls) and underwent no extra handlings. The other half of barren control and enriched control pigs underwent the same procedures as the infected pigs (mock control) at the same days. Instead of PRRSV inoculum and $A$. pleuropneumoniae, mock control pigs received $1.5 \mathrm{~mL}$ RPMI medium and $5 \mathrm{~mL}$ PBS, respectively.

\subsubsection{Blood collection and Enzyme-Linked Immunosorbent Assay}

Blood samples were taken by jugular vein puncture on 7 different days (38, $44,46,48,52,53$, and 54 days of age). Blood was collected in serum separating tubes (Greiner Bio-one, Alphen aan den Rijn, The Netherlands) kept at room temperature (RT) until incubation for $1 \mathrm{~h}$ at $37^{\circ} \mathrm{C}$. After that samples were centrifuged at $5251 \mathrm{~g}$ for $12 \mathrm{~min}$ at $-20^{\circ} \mathrm{C}$. Serum was stored at $-80^{\circ} \mathrm{C}$ until analysis.

$\operatorname{IgM}$ and IgG antibody titers binding with keyhole limpet hemocyanin (KLH), myelin basic protein (MBP, Sigma-Aldrich, St. Louis, MO, USA) or Phosphorylcholine-conjugated to Bovine Serum Albumin (PC-BSA, Santa Cruz Biotechnology, Santa Cruz, CA, USA) were determined by a two-step indirect enzyme-linked immunosorbent assay (ELISA). Medium binding microtiter plates (Greiner) were coated overnight at $4{ }^{\circ} \mathrm{C}$ with $2 \mathrm{mg} / \mathrm{mL} \mathrm{KLH}$ or 0.5 $\mu \mathrm{g} / \mathrm{mL}$ PC-BSA or $0.5 \mu \mathrm{g} / \mathrm{mL} \mathrm{MBP}$ in coating buffer $\left(0.05 \mathrm{M} \mathrm{Na}_{2} \mathrm{CO}_{3} \times 10 \mathrm{H}_{2} \mathrm{O}\right.$, $\mathrm{pH}$ 9.6). Plates were washed with tap water containing $0.05 \%$ Tween 20 , and serial dilutions of serum were added and incubated for $1.5 \mathrm{~h}$ at RT. After washing, plates were incubated for $1.5 \mathrm{~h}$ at RT with a 1:40 000 diluted PO- 
conjugated goat antibody directed to swine $\operatorname{lgM}_{\mathrm{FC}}\left(\mathrm{GASWIgM}_{\mathrm{FC}} / \mathrm{PO}\right.$, Bethyl Laboratories, Montgomery, TX, USA) to detect binding of IgM, or with a 1:40 000 diluted peroxidase (PO)-conjugated goat antibody directed to swine $\operatorname{lgG}_{\mathrm{FC}}$ (GASWlgG $\mathrm{FC} / \mathrm{PO}$, Bethyl Laboratories) to detect binding of IgG, respectively. After washing, tetramethylbenzidine was added as a substrate for 10 min. Reaction was stopped with $2.5 \mathrm{~N} \mathrm{H}_{2} \mathrm{SO}_{4}$ and absorbance was measured at $450 \mathrm{~nm}$ with a Multiskan (Flow, Irvine, UK). Each absorbance was expressed relatively to the absorbance of a standard positive control serum sample, and antibody titers were expressed as $\log _{2}$ values of dilutions that gave extinction closest to $50 \%$ of $E_{\max }$, where $E_{\max }$ represents the highest mean extinction of a standard positive serum present on every microtiter plate.

\subsubsection{Statistical analyses}

SAS (SAS 9.3, SAS Institute Inc.) was used for all statistical analyses. In this experiment, the mock-infected control groups were used to make sure that the procedure to infect the pigs alone did not cause any differences. Preliminary statistical analysis (mixed model) showed no differences in outcome between mock-infected and negative control pigs, so these groups were combined and referred to as "control group".

\section{Overall antibody response}

Antibody titers were analysed using a repeated linear mixed model. The fixed effects of housing, infection, sampling day, and their interactions were included in the model, as well as the random effect of animal. Values in time of individual animals were taken as repeated measurements. All data met the assumptions of the model regarding normal distribution of residuals. 
Subsequently, separate analyses were performed on the housing effect before infection, the housing effect on changes in antibody titers after transport and relocation and the effects of infection and its interaction with housing on changes in antibody titers after infection (see below).

Housing effect on antibody titers before infection

Values on day 38 were used to analyse the effects of housing before transport and infection with a linear mixed model with the fixed effect of housing.

Housing effect on changes in antibody titers after transport and relocation

To investigate housing effects after acute stress due to transport and relocation, the delta between day 44 and day 38 was calculated and subsequently analysed with a linear mixed model with the fixed effect of housing.

Effects of infection and its interaction with housing on changes in antibody titers after infection

To investigate effects of infection, the deltas in titers between day 44 (before PRRSV infection) and days 46,48 , and 52 were calculated for the infection of PRRSV, and the deltas between day 52 (before A. pleuropneumoniae, infection) and days 53 and 54 were calculated for the infection of $A$. pleuropneumoniae, respectively, and separately analysed with a linear mixed model with housing, infection, delta-day and their interaction as fixed effects, and a random effect of animal. Values in time of individual animals were taken as repeated measurements. 
To explore relationships between $N A(A) b$ responses and PRRSV infection characteristics, correlations between basal NA(A)b levels on day 44 (start of infection) and the NA(A)b increase from day 44 to 52 (delta 44-52) with viral RNA measured on 52 of infection, and the decrease in viral RNA from day 48 to 52 were assessed. Data on viral RNA in the same pigs were available from and have been reported in a previous study (van Dixhoorn et al. 2016). In case of significant correlations, differences between correlation coefficients of barren and enriched housed pigs were calculated using Fisher's $r$-to-z transformation. Variables of viral RNA could not be obtained from all 28 infected pigs because of technical problems (van Dixhoorn et al. 2016).

Only significant effects $(p<0.05)$ and tendencies $(p<0.10)$ are reported. Significant interactions were further investigated with post hoc pairwise comparisons using the difference of the least square means with Tukey adjustments. Results are presented as means \pm SEM.

\subsection{Results}

\subsubsection{Natural (auto)-antibody titers}

The course of the antibody titers from day 38 until day 54 is shown in Figure 3.1. Apart from this, the responses to transport and relocation (delta day 44 - day 38) are shown in Table 3.1. Finally, the responses to the PRRSV infection (delta from day $44=$ start of infection) and to A. pleuropneumoniae (delta from day 52 = start of $A$. pleuropneumoniae infection) are shown in Figure 3.2. 


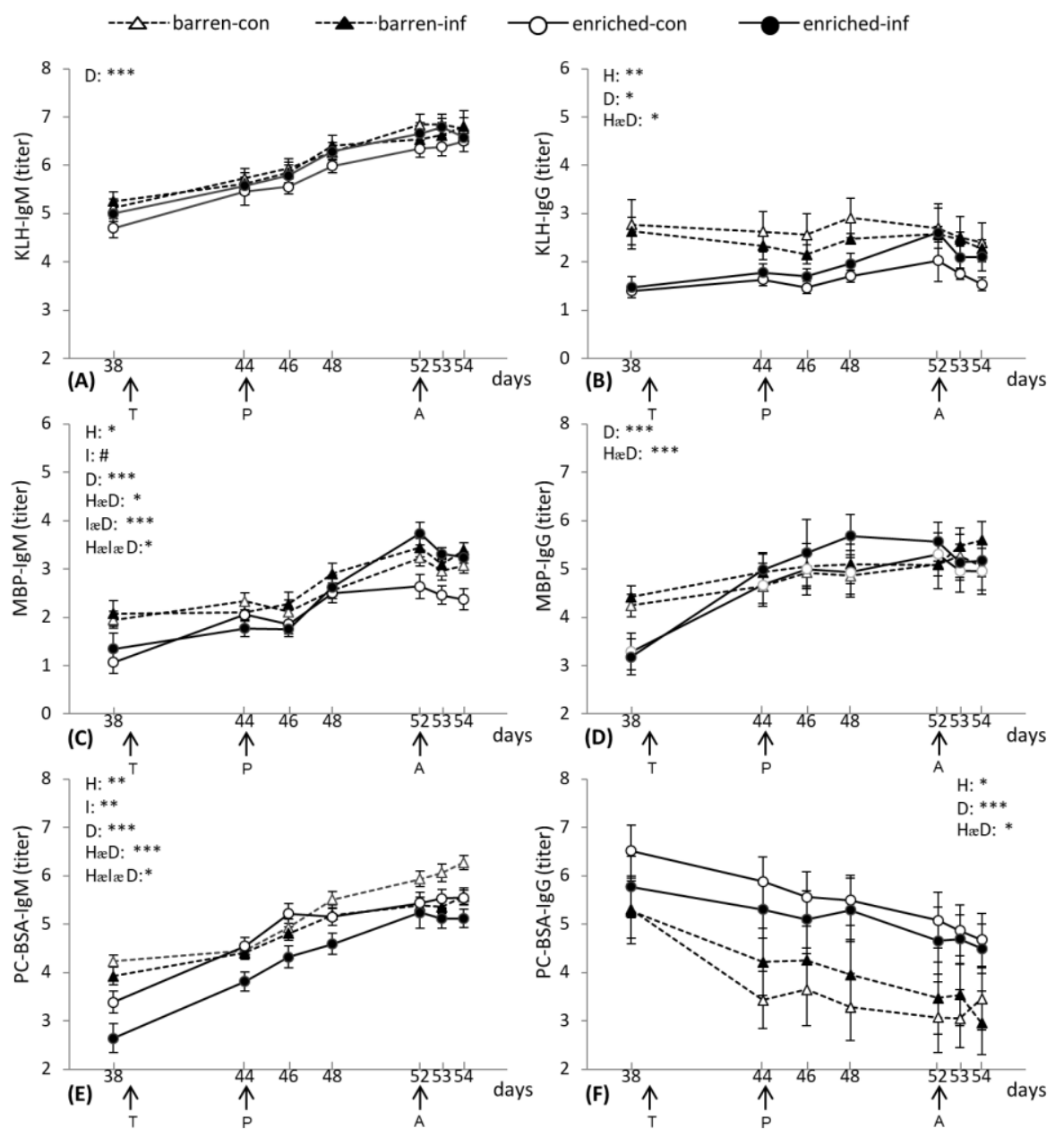

Figure 3.1: Means and SEM of antibody isotype titers. $\lg M(A)$ and $\lg G(B)$ binding $K L H$, IgM $(C)$ and $\lg G(D)$ binding $M B P$, and $\lg M(E)$ and $\lg G(F)$ binding $P C-B S A$ of pigs $(n=56)$ in barren and enriched housing conditions measured on 7 different days. At 39 days of age, pigs were transported to the experimental rooms (T); at 44 days of age, $B I$ and El pigs were infected with PRRSV (P); at 52 days of age, BI and EI pigs were infected with $A$. pleuropneumoniae $(A)$. $H$ indicates the housing effect; I indicates the infection effect; $D$ indicates the sampling day effect. ${ }^{* * *} p<0.001$, ${ }^{* *} p<0.01, * p<0.05, \# p<0.10$. 


\subsubsection{IgM and IgG binding KLH}

KLH-lgM

Overall antibody response. Overall, KLH-IgM titers were not affected by housing or infection, but only by sampling day $\left(F_{(6.307)}=90.8, p<0.001\right)$, with increasing titers from day 38 to 52 (only day 44 and day 46 titers did not differ), but after infection with A. pleuropneumoniae on day 52, levels of KLHIgM remained stable (Figure 3.1A).

Housing effect on antibody titers before infection. On day 38 (before transport), housing tended to affect $\mathrm{KLH}-\operatorname{IgM}\left(\mathrm{F}_{(1.54)}=3.5, \mathrm{p}<0.10\right)$, with higher titers in barren than in enriched housed pigs (Figure 3.1A).

Housing effect on changes in antibody titers after transport and relocation. There was no housing effect on the changes in KLH-IgM before and after transport (Table 3.1).

Table 3.1: Means and SEM of changes (delta 44-38) in antibody isotype titers of barren and enriched housed pigs before and after transport/relocation.

\begin{tabular}{llll}
\hline & barren & enriched & $p$-value \\
\hline KLH-IgM & $0.50 \pm 0.11$ & $0.76 \pm 0.18$ & ns \\
KLH-IgG & $-0.32 \pm 0.19$ & $0.35 \pm 0.11$ & $*$ \\
MBP-IgM & $0.26 \pm 0.13$ & $0.70 \pm 0.15$ & $*$ \\
MBP-IgG & $0.46 \pm 0.21$ & $1.58 \pm 0.31$ & $* *$ \\
PC-BSA-IgM & $0.48 \pm 0.13$ & $1.37 \pm 0.24$ & $* *$ \\
PC-BSA-IgG & $-1.45 \pm 0.22$ & $-0.67 \pm 0.18$ & $* *$ \\
\hline
\end{tabular}

Effects of housing after transport/relocation (delta 44-38) are reported in the text; significances of housing are indicated: ${ }^{*} p<0.01,{ }^{*} p<0.05$, and ns for non-significant.

Effects of infection and its interaction with housing on changes in antibody titers after infection. After infection with PRRSV on day 44, changes of KLH- 
IgM (delta 46-44, 48-44, and 52-44) were not only affected by delta-day $\left(F_{(2.99)}=75.6, p<0.001\right)$, but also by the interaction between infection and delta-day $\left(F_{(2.99)}=3.4, p<0.05\right)$. Post hoc analysis showed that the changes of titers in control pigs significantly increased from $46-44$ to $48-44$ to 52-44, whereas in infected pigs, changes of titers increased from day 46-44 to 4844, with no difference between 48-44 and 52-44 (Figure 3.2A). Besides, changes in KLH-IgM after infection with $A$. pleuropneumoniae tended to be affected by the housing $\times$ infection $\times$ sampling day interaction $\left(F_{(1.50)}=3.1\right.$, $\mathrm{p}<0.10$ ), with no clear pairwise differences in the post hoc analysis (Figure $3.2 A)$.

KLH-IgG

Overall antibody response. Overall, KLH-IgG titers were significantly affected by housing $\left(F_{(1.52)}=7.4, p<0.01\right)$, sampling day $\left(F_{(6.307)}=2.7, p<0.05\right)$, and their interaction $\left(F_{(6.307)}=2.3, p<0.05\right)$. Post hoc analysis showed that only for enriched pigs titers on day 52 were significantly higher than those on

day 46, whereas barren housed pigs showed no day effect on KLH-IgG titers (Figure 3.1B).

Housing effect on antibody titers before infection. Before transport, at 38 days of age, KLH binding IgG titers were higher in barren housed pigs than in enriched housed pigs $\left(F_{(1.54)}=16.5, p<0.001\right.$, Figure 3.1B).

Housing effect on changes in antibody titers after transport and relocation. Housing affected the change of KLH-IgG titers before and after transport (delta 44-38) $\left(F_{(1.54)}=5.0, p<0.05\right)$ (Table 3.1). KLH-IgG increased in enriched housed pigs, whereas KLH-IgG decreased in barren housed pigs (Table 3.1). 
Effects of infection and its interaction with housing on changes in antibody titers after infection. There was no effect of PRRSV nor A. pleuropneumoniae infection on KLH-IgG titers (Figures 3.1B and 3.2B).
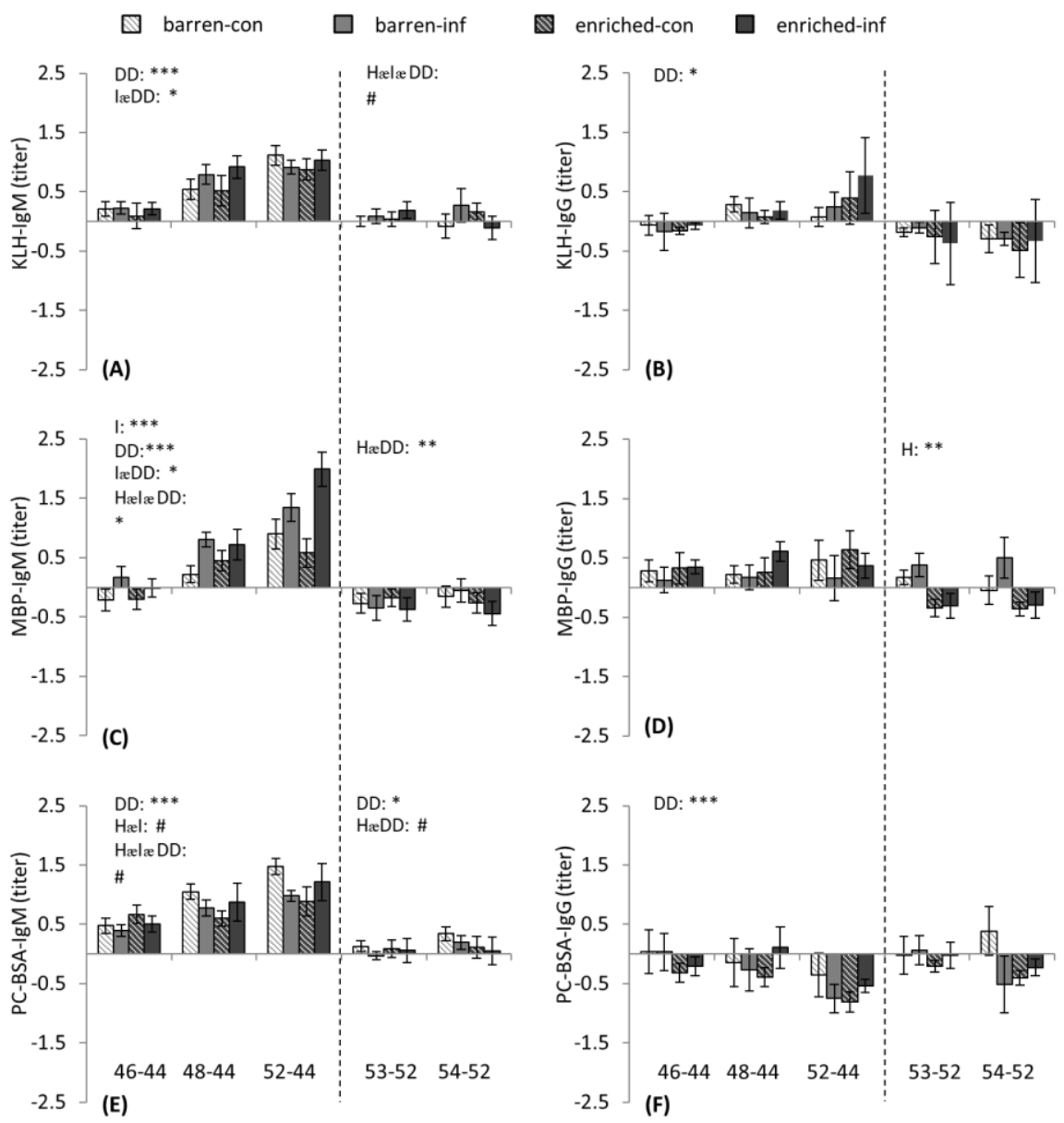

Figure 3.2: Means and SEM of the delta in antibody titers between infection days and sampling days. $\lg M(A)$ and $\lg G(B)$ binding $K L H, \lg M(C)$ and $\lg G(D)$ binding $M B P$, and $\operatorname{IgM}(E)$ and $\lg G(F)$ binding PC-BSA of pigs $(n=56)$ in barren and enriched housing conditions for delta (46-44, 48-44, 52-44, with day 44=PSSRV infection) and delta (53- 
52, 54-52, with day 52=A. pleuropneumoniae infection). The dashed lines divide each graph into the two separate infection conditions. $H$ indicates the housing effect; I indicates the infection effect; $D D$ indicates the delta-day effect. ${ }^{* * *} p<0.001$, ${ }^{* *} p<0.01, * p<0.05, \# p<0.10$.

\subsubsection{IgM and IgG binding MBP}

MBP-IgM

Overall antibody response. Generally, MBP-IgM titers were affected by housing $\left(F_{(1.52)}=3.6, p<0.05\right)$, infection $\left(F_{(1.52)}=4.0, p<0.10\right)$, sampling day $\left(F_{(6,307)}=62.3, p<0.001\right)$, and by the housing $\times$ sampling day $\left(F_{(6.307)}=2.2\right)$, infection $\times$ sampling day $\left(F_{(6.307)}=4.4\right)$, and housing $\times$ infection $\times$ sampling day $\left(F_{(6.307)}=2.2\right)$ interactions $(p<0.05$, see Figure $3.1 C)$.

Housing effect on antibody titers before infection. At 38 days of age, MBP$\lg$ titers were affected by housing $\left(F_{(1.54)}=10.0, p<0.01\right)$, with higher titers in barren compared to enriched housed pigs (Figure 3.1C).

Housing effect on changes in antibody titers after transport and relocation. After transport, the deltas in MBP-IgM titers between day 44 and 38 were affected by housing $\left(F_{(1.54)}=5.4, p<0.05\right)$, as well. MBP-IgM increased more in enriched housed pigs than in barren housed pigs (Table 3.1).

Effects of infection and its interaction with housing on changes in antibody titers after infection. Changes in titers following infection with PRRSV were affected by infection $\left(F_{(1.52)}=15.1, p<0.001\right)$, delta-day $\left(F_{(2.99)}=55.8\right.$, $p<0.001)$, their interaction $\left(F_{(2.99)}=3.8, p<0.05\right)$ and the housing $\times$ infection $\times$ delta-day interaction $\left(F_{(2.99)}=4.0, p<0.05\right)$. Post hoc analysis showed that the increase in MBP-IgM titers following PRRSV infection (delta from titers on 
day 44) was higher between delta 52-44 than between delta $46-44$, with delta 48-44 in between, except for the enriched infected group where titer changes between day 52 and 44 were also higher than those over delta 4844. Thus, only in infected enriched pigs MBP-IgM titers continued to increase from day 48 to day 52. Barren and enriched controls did not differ in titer changes, and barren infected pigs did not differ from barren controls for any time trajectory. Enriched infected pigs, however, had a significantly higher increase in titers from day 44 to day 52 after PRRSV than barren infected pigs, and than both barren and enriched controls (Figure 3.2C).

After infection with A. pleuropneumoniae, there was no infection effect on MBP-IgM deltas (delta 53-52, 54-52), but only of the interaction between housing and delta-day $\left(F_{(1.50)}=9.9, p<0.01\right)$, with a lower decrease in titers from delta 54-52 than from delta 53-52 in barren pigs only (Figure 3.2C).

\section{$M B P-I g G$}

Overall antibody response. Overall, only sampling day $\left(F_{(6.307)}=19.4\right)$ and the interaction between housing and sampling day $\left(F_{(6.307)}=4.2\right)$ affected the levels of MBP binding IgG in the serum $(p<0.001)$. Post hoc analysis showed that in enriched housed pigs titers of MBP-IgG were lower on day 38 than those on each other sampling day, whereas in barren pigs titers on day 38 only differed from those on days 53 , and 54 and tended to differ from titers at day 52 (Figure 3.1D).

Housing effect on antibody titers before infection. On day 38, MBP-IgG titers were higher in barren pigs than in enriched housed pigs (housing effect, $F_{(1.54)}=12.7, p<0.001$, Figure 3.1D). 
Housing effect on changes in antibody titers after transport and relocation. Housing also affected MBP-IgG changes after transport (delta 44-38) $\left(F_{(1.54)}=7.9, p<0.01\right)$, with MBP-IgG titers increasing more in enriched compared with barren housed pigs (Table 3.1).

Effects of infection and its interaction with housing on changes in antibody titers after infection. After infection with PRRSV, there was no housing or infection effect on MBP-IgG, but after infection with A. pleuropneumoniae, there was a housing effect on MBP-IgG $\left(F_{(1.50)}=11.6, p<0.01\right)$, with higher titers in barren than in enriched housed pigs (Figure 3.2D).

\subsubsection{IgM and IgG binding PC-BSA}

\section{$P C-B S A-\operatorname{Ig} M$}

Overall antibody response. Generally, PC-BSA-IgM titers were affected by housing $\left.\left(F_{(1.52)}=4.2\right), p<0.05\right)$, sampling day $\left(F_{(6.307)}=124.0, p<0.001\right)$, their interaction $\left(F_{(6.307)}=5.1, p<0.001\right)$, infection $\left(F_{(1.52)}=13.1, p<0.001\right)$, and the housing $\times$ infection $\times$ sampling day interaction $\left(\mathrm{F}_{(6.307)}=2.8, \mathrm{p}<0.05\right)$ (Figure $3.1 E)$.

Housing effect on antibody titers before infection. At 38 days of age, PC-BSAIgM titers were affected by housing $\left(F_{(1.54)}=21.8, p<0.001\right)$; barren housed pigs had higher levels of PC-BSA-IgM than enriched housed pigs (Figure 3.1E). Housing effect on changes in antibody titers after transport and relocation. Changes in PC-BSA-IgM titers after transport (delta 44-38) were affected by housing $\left(F_{(1.54)}=11.1, p<0.01\right)$ as well; in enriched housed pigs, PC-BSA-IgM titers increased more than in barren housed pigs (Table 3.1). 
Effects of infection and its interaction with housing on changes in antibody titers after infection. After infection with PRRSV, delta PC-BSA-IgM (delta 46$44,48-44$, and $52-44)$ tended to be affected by the interaction between infection and housing $\left(\mathrm{F}_{(1.52)}=3.1, \mathrm{p}<0.10\right)$, by delta-day $\left(\mathrm{F}_{(2.99)}=19.5\right.$, $\mathrm{p}<0.001)$ and by the housing $\times$ infection $\times$ delta-day interaction $\left(\mathrm{F}_{(2.99)}=2.9\right.$, $\mathrm{p}<0.10)$. Post hoc analysis showed that the changes between 52 and 44 were significantly higher than the changes between 46 and 44 for both barren control pigs and enriched infection pigs. There was no infection effect after infection with A. pleuropneumoniae (Figure 3.2E), but an effect of delta-day $\left(F_{(1.50)}=4.0, p<0.05\right)$ and a tendency for a housing $x$ delta-day effect $\left(F_{(1.50)}=3.6, p<0.10\right)$.

$P C-B S A-\lg G$

Overall antibody response. Overall, PC-BSA-IgG titers were affected by sampling day $\left(F_{(6.307)}=32.4, p<0.001\right)$, and their interaction $\left(F_{(6.307)}=2.2\right.$, $p<0.05)$. Unlike the other antibodies, titers of PC-BSA-IgG decreased over time (Figure 3.1F). Post hoc analysis showed that for both barren and enriched groups, levels of PC-BSA-IgG on day 38 were higher than on other sampling days.

Housing effect on antibody titers before infection. There was no housing effect on PC-BSA-IgG on day 38.

Housing effect on changes in antibody titers after transport and relocation. After transport, the delta PC-BSA-IgG values between day 44 and day 38 were affected by housing $\left(F_{(1.54)}=9.0, p<0.01\right)$. PC-BSA-lgG decreased less in enriched housed pigs than in barren housed pigs (Table 3.1). 
Effects of infection and its interaction with housing on changes in antibody titers after infection. There was no infection effect (Figure 3.1F and Figure $3.2 \mathrm{~F})$.

\subsubsection{Relationships between viral clearance and NA(A)b}

The titers of IgG NA(A)b and IgM NA(A)b except MBP-IgM on day 44 and their increase until day 52 did not correlate with viral RNA levels. Increasing MBPIgM levels (delta 52-44) were positively correlated with the decrease in viral RNA from day 48 to day $52(R=0.53, p<0.05$; see Figure 3.3). However, the latter relationship was stronger in barren $(R=0.66, p<0.05)$ than in enriched housed pigs $(R=-0.27, p>0.10)$ as the two correlation coefficients significantly differed $(p<0.05)$. No other correlations were found.

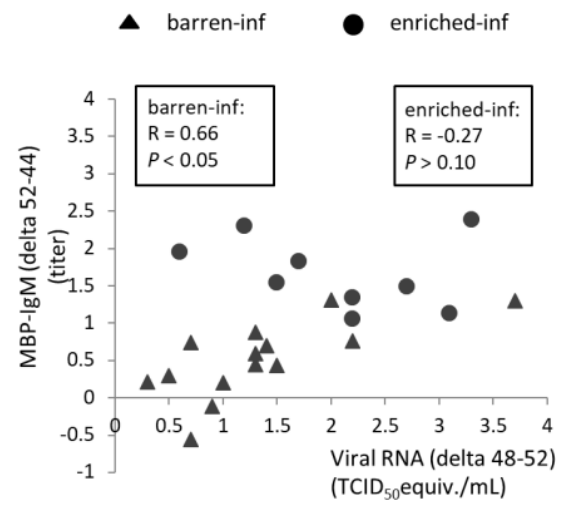

Figure 3.3: The correlation between viral clearance and $N A(A) b$. The correlation between the changes of MBP-IgM from day 44 to day 52 and the reduction of viral RNA from day 48 to day 52 for barren and enriched pigs infected with PRRSV. 


\subsection{Discussion}

In this study, we investigated the effect of environmental enrichment versus barren housing on the level of $N A(A) b$ binding $K L H, M B P$, and PC-BSA prior to transport and relocation, after transport and after infection with PRRSV and A. pleuropneumoniae. In general, all IgM antibody titers increased over time, which may reflect an aging effect (Reimert et al. 2014). It has been proposed that $\operatorname{lgM}$ antibodies binding auto-antigens such as PC are usually present, and enough IgM is available to prevent inflammation, for instance by activating the classical complement pathway (Lobo 2016), and consequently IgG is not formed. In the current study, IgG binding to PC-BSA decreased over time, which is in line with the previous study (Luo et al. 2017). Whether this also reflected decreasing maternal IgG antibodies remains unknown. IgM antibodies in pigs are most likely not from maternal origin, thus housing effects and infection effects on IgM most likely reflect effects on the pigs' immune system. Our study shows that housing before transport affected $N A(A) b$ levels in the blood, with generally significantly higher levels of IgM and IgG binding KLH, MBP and PC-BSA in barren housed pigs. Infection only affected IgM, but not IgG natural (auto-)antibodies, and did so in a housingdependent manner.

\subsubsection{Housing effects}

Housing or its interaction with sampling day affected all NA(A)b levels except KLH-IgM. In line with this, effects of housing on NA(A)b binding KLH (IgG, (Reimert et al. 2014)), MBP and PC-BSA (IgM, (Luo et al. 2017)) have previously been found. Notably, before infection, barren housed pigs had higher levels of NA(A)b than enriched housed pigs, except for PC-BSA-IgG. 
This is in contrast with previous studies in which generally lower NA(A)b titers were found for barren housed pigs, with the exception of PC-BSA-IgG (Luo et al. 2017; Reimert et al. 2014). There could be several reasons for this discrepancy. First, in the present experiment, pigs were housed in enriched or barren conditions, starting directly after birth instead of starting after weaning. Therefore, pigs in the present study had a longer experience with enriched conditions than the pigs in the previous studies. Second, unlike the previous experiments, enriched housed pigs in the present study were not only provided with ample substrates to root in, but also with extra space, which seems to be an important enrichment factor (Cornale et al. 2015; Vermeer et al. 2014). Finally, blood samples were taken at a younger age as compared to previous studies.

The changes in antibody titers from day 38 (before transport and relocation) to day 44 , just before infection (delta transport effect) were significantly different between barren and enriched housed pigs too. Higher deltas (higher increases and lower decrease) were found in the enriched housed pigs, whereas antibody titers on day 38 and 44 were not much different for barren housed pigs, which already had higher titers on day 38. It can be speculated that the increases in antibody titers found in particularly enriched housed pigs is related to the stress of transport and relocation experienced by these pigs, as we previously found increased anti-PC-BSA antibody titers after regrouping stress (Luo et al. 2017), but age effects cannot be fully excluded. In our previous study, however, both barren and enriched housed pigs showed such increases following regrouping (Luo et al. 2017).

\subsubsection{Infection effects}


Effects of infection with PRRSV on the titers on day 44 up till day 52 were found for IgM binding MBP and PC-BSA. Lobo et al. (Lobo et al. 2008a; Lobo et al. 2008b) also found that IgM binding leukocytes, but not IgG can limit HIV infection in an antigen-nonspecific fashion. The reason could be that the functional activity of IgG is blocked by poly-reactive IgM with anti-idiotypic activity (Adib et al. 1990). It has been proposed that natural IgG-producing B cells, unlike natural IgM-producing B cells, are in an inactive state at birth, and these $B$ cells start to produce IgG after exposure to bacterial or foreign antigens (Coutinho et al. 1995; Pereira et al. 1986), and thus it may take a long time before significant levels of IgG can be detected (Hamanova et al. 2015). It is, therefore, not surprising that the effect of IgG on infection was not detected within the short infection period in this study. Remarkably, in the current study, the effects of PRRSV infection on IgM NA(A)b titers were housing-dependent. Only in infected enriched pigs, MBP-IgM titers continued to increase from day 4 to day 8 after infection (day 48 to 52 of age), and infected pigs from enriched housing showed a higher increase in titers starting directly after PRRSV until 8 days after infection than controls and barren infected pigs. Thus, enriched infected pigs showed a higher and more prolonged increase from infection onwards in MBP-IgM. A previous study on the same pigs revealed that infection characteristics differed for barren and enriched housed pigs. Four days after PRRSV infection (day 48) viral RNA amount was equally increased in both barren infected and enriched infected pigs, whereas 8 days after infection (day 52), enriched infected pigs had significantly lower viral RNA. Moreover, enriched housed pigs were subsequently less likely to develop lung lesions following $A$. pleuropneumoniae infection (van Dixhoorn et al. 2016). 
Remarkably, in barren, but not enriched housed pigs a correlation was found between the increase in MBP-IgM antibody titers from day 44 to 52 and the reduction in viral RNA between day 48 and 52 . Thus, barren housed pigs with a relatively higher increase in MBP-IgM following infection, showed a faster reduction of viral RNA in serum. This correlation was not found in enriched pigs, who had both already higher MBP-IgM titers (in this study) and a faster reduction in viral RNA (van Dixhoorn et al. 2016). Whether or not the IgM $N A(A) b$ play a role in defence against the virus, remains to be elucidated.

Pigs were sacrificed shortly after A. pleuropneumoniae infection on day 52, and no effect of infection of the bacteria was found on the changes in NA(A)b up till day 54, except for KLH-IgM.

The results of the current study are difficult to explain. $N A(A) b$ levels have been proposed to be independent from the environment (Lobo 2016). However, in the current study, NA(A)b, particularly of the IgM isotype, binding all three antigens were affected by housing conditions, and, moreover infection with PRRSV affected MBP-IgM NA(A)b in a housingdependent manner. Our data suggested that environmental enrichment may facilitate immune sensitivity in a non-antigen specific fashion resulting in higher NA(A)b levels following viral infection. In poultry, levels of auto-lgM showed a relatively high heritability as opposed to auto-IgG suggesting that IgM is less affected by the environment than IgG (Bao et al. 2016). It remains to be elucidated whether the higher or lower antibody levels in the present study with pigs reflect the usage of antibody in response to cell or tissue damage due to stress as a homeostatic response (Lutz et al. 2009), or reflect a non-antigen specific B-cell activation (Lobo 2016). Either way, this study 
suggests that levels of auto-antibodies including IgM, are affected by the environment (housing) and/or infection, and therefore may provide additional information of the physiological response of pigs to stress in general. Remarkably, the enriched housed pigs were less prone to develop lesions caused by a combination of PRRSV and A. pleuropneumoniae (van Dixhoorn et al. 2016). Whether this rests on a higher sensitivity of NA(A)b to the environment or changing $N A(A) b$ levels reflect sensitivity of the individual to the environment remains to be established.

\subsection{Conclusion}

In conclusion, type of housing affected serum levels of $N A(A) b$ binding KLH, MBP and PC-BSA. Infection with PRRSV only affected the IgM, but not the IgG isotype. In this study, barren housed pigs had higher levels of NA(A)b, except for PC-BSA-IgG, than enriched housed pigs before transport and infection. However, NA(A)b levels of enriched housed pigs responded more to transport and relocation stress than barren housed pigs. Besides, enriched and barren housed pigs responded differently to PRRSV infection. NA(A)b may thus be affected by infection, and the effect of infection on NA(A)b is influenced by housing conditions. More research is warranted to investigate the mechanisms by which environmental enrichment affects $N A(A) b$ and the role of $N A(A) b$ in the defence of infection.

\subsection{Competing interests}

The authors declare that they have no competing interests. 


\subsection{Authors' contributions}

$\mathrm{LL}$ analysed data and wrote the manuscript. IDE van D designed and carried out the animal experiments. IR and JEB helped analyse data and revise the manuscript. HKP participated in the design of the study. BK helped revise the manuscript. All authors read and approved the final manuscript.

\subsection{Acknowledgements}

The authors would like to thank Mike Nieuwland for assistance with laboratory analyses. 


\subsection{References}

Abou-Donia MB, Abou-Donia MM, EIMasry EM, Monro JA, Mulder MF (2013) Autoantibodies to nervous system-specific proteins are elevated in sera of flight crew members: biomarkers for nervous system injury Journal of Toxicology and Environmental Health, Part A 76:363-380

Adib M, Ragimbeau J, Avrameas S, Ternynck T (1990) IgG autoantibody activity in normal mouse serum is controlled by $\operatorname{lgM}$ The Journal of Immunology 145:3807-3813

Bao M, Bovenhuis H, Nieuwland M, Parmentier H, van der Poel J (2016) Genetic parameters of IgM and IgG antibodies binding autoantigens in healthy chickens Poultry science 95:458-465

Bolhuis JE, Schouten WG, Schrama JW, Wiegant VM (2005) Behavioural development of pigs with different coping characteristics in barren and substrate-enriched housing conditions Applied Animal Behaviour Science 93:213-228

Bolhuis JE, Schouten WG, Schrama JW, Wiegant VM (2006) Effects of rearing and housing environment on behaviour and performance of pigs with different coping characteristics Applied Animal Behaviour Science 101:68-85

Carreras R et al. (2016) Housing conditions do not alter cognitive bias but affect serum cortisol, qualitative behaviour assessment and wounds on the carcass in pigs Appl Anim Behav Sci 185:39-44

Cornale P, Macchi E, Miretti S, Renna M, Lussiana C, Perona G, Mimosi A (2015) Effects of stocking density and environmental enrichment on behavior and fecal corticosteroid levels of pigs under commercial farm conditions Journal of Veterinary Behavior: Clinical Applications and Research 10:569-576

Coutinho A, Kazatchkine MD, Avrameas S (1995) Natural autoantibodies Current opinion in immunology 7:812-818

de Groot J, de Jong IC, Prelle IT, Koolhaas JM (2000) Immunity in barren and enriched housed pigs differing in baseline cortisol concentration Physiology \& behavior 71:217-223

de Jong IC, Prelle IT, van de Burgwal JA, Lambooij E, Korte SM, Blokhuis HJ, Koolhaas JM (2000) Effects of environmental enrichment on behavioral responses to novelty, learning, and memory, and the circadian rhythm in cortisol in growing pigs Physiology \& behavior 68:571-578 
Diamond MS, Shrestha B, Marri A, Mahan D, Engle M (2003) B cells and antibody play critical roles in the immediate defense of disseminated infection by West Nile encephalitis virus Journal of virology 77:25782586

Ehrenstein MR, Notley CA (2010) The importance of natural IgM: scavenger, protector and regulator Nature Reviews Immunology 10:778-786

Ernst K, Tuchscherer M, Kanitz E, Puppe B, Manteuffel G (2006) Effects of attention and rewarded activity on immune parameters and wound healing in pigs Physiology \& behavior 89:448-456

Gao J et al. (2012) Novel functions of murine B1 cells: active phagocytic and microbicidal abilities European journal of immunology 42:982-992

Hamanova M, Chmelikova M, Nentwich I, Thon V, Lokaj J (2015) Anti-Gal IgM, IgA and IgG natural antibodies in childhood Immunology letters 164:40-43

Lévesque C, Provost C, Labrie J, Reyes YH, Nava JAB, Gagnon CA, Jacques M (2014) Actinobacillus pleuropneumoniae possesses an antiviral activity against porcine reproductive and respiratory syndrome virus PLoS One 9:e98434

Lobo PI (2016) Role of Natural Autoantibodies and Natural IgM AntiLeucocyte Autoantibodies in Health and Disease Frontiers in Immunology 7

Lobo PI et al. (2008a) Naturally occurring IgM anti-leukocyte autoantibodies (IgM-ALA) inhibit T cell activation and chemotaxis The Journal of Immunology 180:1780-1791

Lobo PI, Schlegel KH, Yuan W, Townsend GC, White JA (2008b) Inhibition of HIV-1 infectivity through an innate mechanism involving naturally occurring IgM anti-leukocyte autoantibodies The Journal of Immunology 180:1769-1779

Luo L, Geers R, Reimert I, Kemp B, Parmentier H, Bolhuis J (2017) Effects of environmental enrichment and regrouping on natural autoantibodies-binding danger and neural antigens in healthy pigs with different individual characteristics animal:1-8

Lutz HU, Binder CJ, Kaveri S (2009) Naturally occurring auto-antibodies in homeostasis and disease Trends in immunology 30:43-51

Merlot E et al. (2012) Health and immune traits of Basque and Large White pigs housed in a conventional or enriched environment animal 6:1290-1299

Ochsenbein AF, Fehr T, Lutz $C$, Suter $M$, Brombacher $F$, Hengartner $H$, Zinkernagel RM (1999) Control of early viral and bacterial 
distribution and disease by natural antibodies Science 286:21562159

Panda S, Zhang J, Tan NS, Ho B, Ding JL (2013) Natural IgG antibodies provide innate protection against ficolin-opsonized bacteria The EMBO journal 32:2905-2919

Pereira P, Forni L, Larsson EL, Cooper M, Heusser C, Coutinho A (1986) Autonomous activation of $B$ and $T$ cells in antigen-free mice European journal of immunology 16:685-688

Proudfoot K, Habing G (2015) Social stress as a cause of diseases in farm animals: current knowledge and future directions The Veterinary Journal 206:15-21

Quartier P, Potter PK, Ehrenstein MR, Walport MJ, Botto M (2005) Predominant role of IgM-dependent activation of the classical pathway in the clearance of dying cells by murine bone marrowderived macrophages in vitro European journal of immunology $35: 252-260$

Reimert I, Rodenburg TB, Ursinus WW, Kemp B, Bolhuis JE (2014) Selection based on indirect genetic effects for growth, environmental enrichment and coping style affect the immune status of pigs PloS one 9:e108700

Scott K, Taylor L, Gill BP, Edwards SA (2009) Influence of different types of environmental enrichment on the behaviour of finishing pigs in two different housing systems: 3 . Hanging toy versus rootable toy of the same material Appl Anim Behav Sci 116:186-190

Telkänranta H, Bracke MB, Valros A (2014) Fresh wood reduces tail and ear biting and increases exploratory behaviour in finishing pigs Applied Animal Behaviour Science 161:51-59

van Dixhoorn ID et al. (2016) Enriched housing reduces disease susceptibility to co-infection with porcine reproductive and respiratory virus (PRRSV) and Actinobacillus pleuropneumoniae (A. pleuropneumoniae) in young pigs PloS one 11:e0161832

Vermeer H, De Greef K, Houwers H (2014) Space allowance and pen size affect welfare indicators and performance of growing pigs under Comfort Class conditions Livestock Science 159:79-86 



\section{Chapter 4}

\section{Effects of early and current environmental enrichment on behaviour and growth in pigs}

L. Luo, I. Reimert, A. Middelkoop, H. K. Parmentier, B. Kemp, J. E. Bolhuis*

Adaptation Physiology Group, Department of Animal Sciences, Wageningen University \& Research, PO Box 338, 6700 AH, Wageningen, the Netherlands

*Corresponding author: J. E. Bolhuis

E-mail address: liesbeth.bolhuis@wur.nl 


\subsection{Abstract}

Enriched environments are known to beneficially affect the behaviour of pigs, as compared with more barren pens. The influence of enrichment may, however, depend on pigs' early life housing experiences. Aim of this study was to investigate the (long-term) effects of early and later life environmental enrichment on behaviour and growth in pigs with different coping styles. Pigs were housed in either barren or enriched housing conditions from birth, and half of them experienced a housing switch, i.e. a loss or gain of enrichment, at 7 weeks of age, creating four treatment groups. Enriched pens were larger than barren pens and contained peat, straw, sawdust and extra toys. Home pen behaviour and body weight were recorded up till 19 weeks of age. Pigs were classified as reactive or proactive based on a backtest at two weeks of age. Enrichment increased time spent exploring, chewing and play, and decreased damaging behaviours and pendirected exploring and chewing. Behaviour of pigs that switched from barren to enriched pens or vice versa reflected, not only their actual environment, but was also influenced by early life housing conditions. Effects of early and later life enrichment on most behaviours were in opposite direction. The effects of (lack of) enrichment after the switch were therefore more pronounced in pigs that had experienced a different early life condition. For instance, pigs experiencing an upgrade from barren to enriched pens seemed to 'catch up' by showing more exploration and play. Conversely, pigs exposed to a downgrade displayed more oral manipulation of pen mates than ones kept barren throughout, which particularly held for pigs with a reactive coping style. Effects of early life and current housing on several other behaviours depended on coping style as well. Pigs housed in enriched 
conditions appeared better able to cope with weaning, as they gained more weight and had higher feed intake post-weaning. Barren housed pigs had a lower body weight than enriched pigs just before the switch, after which growth was mainly determined by actual housing, with enriched kept pigs having a higher feed intake and body weight. Thus, not only current housing conditions, but also a (mis)match with the early life environment may affect behaviour and growth of pigs.

Keywords: pigs, behaviour, environmental enrichment, growth, early life 


\subsection{Introduction}

Pigs in intensive farming conditions are often housed in stimulus-poor, barren environments, which offer little potential to facilitate their natural species-specific behaviours. Consequently, the limited living space, and the lack of materials for foraging and exploration in barren housing conditions are major risk factors for the development of damaging oral behaviours, such as tail biting and ear biting (Beattie et al. 2000a; Kallio et al. 2018; Moinard et al. 2003; Petersen et al. 1995; Scollo et al. 2016; Taylor et al. 2012). Moreover, these barren conditions can also cause chronic stress in pigs, as reflected in physiological changes (Carreras et al. 2016; Chaloupková et al. 2007b; de Jong et al. 1998). In addition, barren housing conditions were found to alter immunity (Luo et al. 2017a; Luo et al. 2017b; Reimert et al. 2014) and even to increase the susceptibility to lung infections (van Dixhoorn et al. 2016).

According to a European Commission Directive (2001/93/EC), pigs "must have permanent access to a sufficient quantity of materials to enable proper investigation and manipulation activities", with the intention to improve the welfare of pigs. Numerous studies have proven that, as opposed to barren housing, enrichment of the environment with such materials (such as straw bedding or peat) can reduce damaging behaviours (e.g Bolhuis et al. 2005a; Bolhuis et al. 2006; Telkänranta et al. 2014; Van de Weerd et al. 2005; Van de Weerd et al. 2006), and increase play behaviour in pigs (e.g. Bolhuis et al. 2005a; Bolhuis et al. 2006; Chaloupková et al. 2007a). Enriched housed pigs were also more active (Beattie et al. 2000b; Bolhuis et al. 2005a) and showed more explorative behaviour (Averós et al. 2010; Bolhuis et al. 2005a). Besides, some studies report enhanced growth rates in pigs kept in enriched 
environments (e.g. Beattie et al. 1995; Bolhuis et al. 2006; Munsterhjelm et al. 2009), but see Camerlink et al. (2014); Morrison et al. (2007) who did not find such an effect.

Apart from the current conditions in which pigs are housed, also the environment in earlier life stages could have an effect on their later life behaviour and welfare. It has been shown in several species that adverse early life experiences can have negative long-term effects on behaviour, physiology, and cognition. For instance, social isolation in early life increased anxiety and conditioned fear behaviours in adult rats (Lukkes et al. 2009), and limited space before weaning negatively influenced the social skills needed in later life in pigs (Lammers and Schouten 1985). Furthermore, it has been shown that the environment of pigs in the farrowing phase can affect the risk of tail biting in later life (e.g. Moinard et al. 2003; Simonsen 1995). If a piglet does not have enough opportunity to explore the environment in early life, it is possible that this exploration may be directed towards its pen mates (Munsterhjelm et al. 2009; Ursinus et al. 2014), thus posing a risk for the development of adverse behaviours which may persist into later life. Conversely, environmental enrichment may have beneficial effects on later life functioning, putatively via its effect on brain development and functioning (do Prado et al. 2016; Kuzumaki et al. 2011; Mora et al. 2007). In pigs, for instance, rearing conditions consisting of an outdoor pasture with loose housed sows in the first 6 weeks of life suppressed the development of social stress in adult life (De Jonge et al. 1996), and pre-weaning substrateenrichment, in the form of wood shavings and chopped straw, decreased the number of agonistic encounters at a later age (Munsterhjelm et al. 2009). 
Hence, the behaviour of pigs may, apart from being affected by their current environment, also be shaped by early life experiences. On the one hand, as outlined above, an enriched environment in early life, as opposed to barren rearing conditions, may have long-term beneficial effects, protecting animals against developing aberrant behaviours. For example, mice reared in large, enriched cages showed less stereotypic behaviours when switched to standard cages than those housed in standard conditions all their lives (Hadley et al. 2006; Powell et al. 2000). On the other hand, removal of enrichment in later life might also lead to behavioural changes indicative of frustration, as animals originating from an enriched environment could be less satisfied by the poor resources in barren housing conditions. In pigs, results of some studies suggest that experience of loss of enrichment could be more detrimental than housing in barren conditions throughout life. For example, Day et al. (2002) found that moving pigs from straw-bedded to barren pens increased the occurrence of damaging pen-mate-directed behaviour compared to pigs without experience with straw. Less is known about the influence of switching from relatively barren to enriched housing, although Bøe (1993) found that pigs that were transferred from flat deck weaner pens with slatted floors to grower pens bedded with sawdust showed more rooting and chewing of the bedding than pigs originating from bedded pens. This could be interpreted as a (short-term) 'catching up' effect indicating an increased motivation for exploration in pigs that had been thwarted in the expression of this behaviour before (Bolhuis et al. 2006). It is largely unknown whether these potential 'frustration' or 'catching up' effects, resulting from a change from enriched to barren housing or vice versa, are transient or may sustain, and hence affect welfare for a longer time. 
The impact of the environment on behaviour may differ for pigs varying in coping style, a personality trait (e.g. Bolhuis et al. 2005a). Individuals with a 'proactive' coping style show a more active behavioural stress response and are prone to develop routines, whereas individuals with a 'reactive' coping style tend to explore novel environments for longer, are more flexible and more attentive to subtle environmental changes. Pigs with a proactive personality seem to have more trouble in adapting to an environmental change (Bolhuis et al. 2004; Koolhaas et al. 1999) which could be related to their lower flexibility in behaviour as compared with reactive pigs (Bolhuis et al. 2004; Bolhuis et al. 2005b). On the other hand, reactive pigs may be more influenced by their long-term housing environment, as several studies reported that they were more affected by (the absence of) enrichment than proactive pigs (Asher et al. 2016; Bolhuis et al. 2003; Bolhuis et al. 2004), although we recently found that the behaviour of proactive pigs in an attention bias test was more influenced by enrichment (Luo et al. 2019a). Thus, the impact of enrichment and a loss or gain of enrichment may depend on the personality characteristics of the pig under study, which may therefore be relevant to take into account.

The aim of this study was to investigate the (long-term) effects of early and later life environmental enrichment on behaviour and growth in pigs with different coping styles. To that aim, pigs were housed in either barren or enriched housing conditions from birth, and half of them experienced a housing switch, i.e. a loss or gain of enrichment. Behaviour and body weight development were recorded up till 19 weeks. 


\subsection{Materials and Methods}

The established principles of laboratory animal use and care were followed, as well as the Dutch law on animal experiments. The Animal Care and Use Committee of Wageningen University \& Research approved the experiment.

\subsubsection{Animals and housing before the housing switch}

Pigs (Tempo $\times$ Topigs 20) from 30 litters, equally divided over 2 batches, were studied in this experiment. Multiparous Topigs 20 sows (parity: $4.1 \pm 0.9$ ) were inseminated on the same day in each batch, and were housed in Carus, the animal facilities of Wageningen University \& Research, Wageningen, the Netherlands, from one month before farrowing. One week before the expected farrowing date, they were moved to individual farrowing pens. Distribution of sows over the housing treatments for their piglets (see below) was balanced for parity and sow weight and back fat after arrival. The maximum litter size was 14 , and piglets were cross fostered within treatment if litter sizes were larger than 14. Litter size at day 7 (enriched: $12.7 \pm 0.2$, barren: $12.5 \pm 0.4$ piglets/litter), litter size at weaning (enriched: $12.4 \pm 0.2$, barren: $11.9 \pm 0.4$ piglets/litter), birth weight (enriched: $1.48 \pm 0.05$, barren: $1.52 \pm 0.05 \mathrm{~kg}$ ) and weaning age (enriched: $29.7 \pm 0.4$, barren: $29.7 \pm 0.4$ days of age) did not differ between treatments (see below).

From birth till weaning, half of piglets were housed in barren $\left(B, 8.6 \mathrm{~m}^{2}\right)$ pens with a solid floor and a small slatted area for drain, with their sow in a farrowing crate. The other half were housed in enriched $\left(E, 17.1 \mathrm{~m}^{2}\right)$ pens to which, apart from the $8.6 \mathrm{~m}^{2}$ area with the sow crated as described for the $B$ pens, an additional enriched part was added with $1.7 \mathrm{~kg}$ straw, $300 \mathrm{~L}$ of sawdust, and $270 \mathrm{~L}$ of peat as substrates on the floor. Besides, $0.8 \mathrm{~kg}$ fresh 
straw and $40 \mathrm{~L}$ of fresh sawdust were added daily, and $30 \mathrm{~L}$ of fresh peat was added weekly in the enriched part of the pen. Additionally, two fixed objects, here referred to as toys (one chain with a ball and one chain with screws that touched floor), were placed in the B pens. In the E pens, one fixed toy (a chain with a ball) and a toy that was alternated daily and selected from 4 different toys, were placed.

Each pen had one drinking nipple for the piglets and one for the sow. Sows were fed a standard commercial diet twice a day. From 5 days of age until day 22, some fresh creep feed (Prestarter Speen Select, AgruniekRijnvallei, Wageningen, The Netherlands) was provided for the piglets, which was mixed with the weaner feed (Speen Uniek VC, AgruniekRijnvallei) from day 23 until weaning. The room temperature was set at $25^{\circ} \mathrm{C}$, and was gradually decreased to $21^{\circ} \mathrm{C}$ over the course of two weeks. In the first week after birth, one heating lamp was provided in each B pen, and two lamps in each $E$ pen. Each pen was cleaned daily and lights and a radio were on from 7:00 until 19:00 hours. Even though sows were all inseminated on the same day, farrowing was spread over a number of days. Procedures and observations below were all conducted on the same day, and days of age as referred to in the paper (except for weight at the day of birth) all relate to the number of days after the expected day of farrowing.

At 13 days of age, all pigs were subjected to a backtest to assess their coping style (Bolhuis et al. 2000; Koolhaas and Van Reenen 2016). Briefly, in this test, piglets are restrained in supine position for $1 \mathrm{~min}$ and the number and latency of escape attempts and vocalisations are recorded (see Melotti et al. 2011 for details). Pigs were classified as relatively "high resisters" (HR) if they 
struggled 2 times and vocalized at least 25 times, or struggled at least 3 times, and as "low resisters" (LR) if they struggled 0 or 1 time, or struggled 2 times and vocalized less than 25 times (Reimert et al. 2014). Thus, not the extremes were selected, but the population was split into two classifications. There was no effect of housing on the proportion of HR and LR pigs (data not shown).

At 28 days of age, pigs were weaned, and in total 192 pigs (96 per batch) were selected and regrouped in 32 new pens containing 6 non-littermate pigs each. All pigs were equally regrouped by taking sex ( 3 males and 3 females), coping style ( 3 HR and 3 LR) and body weight into account. Housing treatment ( $B$ vs. E) for each pig was kept the same as before weaning. After weaning, therefore, the pigs from B farrowing pens were moved to barren pens $\left(5.6 \mathrm{~m}^{2}\right)$, with partly solid floor and partly slatted floor. The pigs from $E$ farrowing pens were moved to enriched pens $\left(11.2 \mathrm{~m}^{2}\right)$ with $2.5 \mathrm{~kg}$ straw, $400 \mathrm{~L}$ of sawdust, and $360 \mathrm{~L}$ of peat on the floor. Additionally, $1.25 \mathrm{~kg}$ fresh straw and $60 \mathrm{~L}$ fresh sawdust were added daily, and $45 \mathrm{~L}$ fresh peat was added weekly in the enriched pens. The toys in the barren and enriched pens were kept the same as before weaning, and from 39 days of age, enriched housed pigs received extra enrichment such as, for instance, a jute sack, a rope, branches, a log of wood or an egg tray on each Monday until the end of the experiment (day 133). The housing conditions before the switch (see below) are labelled with a "1" (i.e. B1 or E1).

Each pen had one drinking nipple and pigs received standard commercial solid feed ( 0 - 10 days after weaning: Speen Uniek VC; 11 - 34 days after weaning: Babybiggen Uniek VC, AgruniekRijnvallei; from 35 days after 
weaning onwards: Start Uniek, AgruniekRijnvallei) ad libitum. On the weaning day, the room temperature was set at $25^{\circ} \mathrm{C}$, and it was gradually decreased to $21{ }^{\circ} \mathrm{C}$ over the course of two weeks and kept at that temperature until the end of the experiment. After weaning, one heating lamp was provided for the first two weeks. Lights and a radio were on from 7:00 until 19:00 hours.

\subsubsection{Housing after the switch}

At 47 days of age, half of the groups of pigs experienced a switch in housing type, and the other half did not. All groups of pigs, including the ones that did not change housing type, were moved to new pens, and group composition remained the same. Thus, after the switch, there were four housing treatment groups: E1E2, E1B2, B1E2, B1B2, $n=8$ pens per group (192 pigs in total), with 1 and 2 reflecting the housing conditions before and after the switch, respectively. Straw, peat and toys were used and added as described before, but only $30 \mathrm{~L}$ of sawdust was added daily in the E2 pens.

Pigs were, both before and after weaning, housed in two rooms per batch. Distribution of (early and later life) barren and enriched pens over the rooms was balanced. Part of the pigs within a pen were exposed to tests for emotional state and immunity, results of which are published elsewhere (Luo et al. 2019a; Luo et al. under review; Luo et al. in preparation). This was balanced for the treatments.

\subsubsection{Behaviour observations}

Behaviour (Table 4.1) of individual pigs before weaning, in the farrowing pens, was scored live using 4-min instantaneous scan sampling for $3 \mathrm{~h}$ per day at 
20 and 21 days of age (in total, $6 \mathrm{~h}$ per pen before weaning). Behaviour (Table 4.1) of individual pigs after weaning was scored live using 2.5-min instantaneous scan sampling for $6 \mathrm{~h}$ per day at 45 ( 2 days before the switch), 49 ( 2 days after the switch), 54 ( 7 days after the switch), 60 (13 days after the switch), 78 (31 days after the switch), and 125 days of age (78 days after the switch). Observation hours started at 8:00 h, 9:15 h, 10:30 h, 14:00 h, 15:15 $\mathrm{h}$ and 16:30 h. These procedures resulted in a total of 90 observations per pig before weaning, and 144 observations per pig per observation day after weaning. On each observation day, there was no activity in the rooms other than daily cleaning. Observers were always balanced over treatments and changed rooms every hour. Agreement between the different observers was substantial (before weaning: average Cohen's Kappa $(k)=0.75$ (range: 0.710.79), after weaning: $k=0.77$ (0.71-0.90) (Landis and Koch 1977; Martin and Bateson 2007). 


\section{Table 4.1: Ethogram used for the behaviour observations.}

\begin{tabular}{|c|c|}
\hline Behaviour & Definition \\
\hline $\begin{array}{l}\text { Inactive } \\
\text { behaviour }\end{array}$ & $\begin{array}{l}\text { Lying on side or belly with eyes closed or open and without performing any other described } \\
\text { behaviour and eating or drinking behaviour. }\end{array}$ \\
\hline Social behaviour & $\begin{array}{l}\text { Touching or sniffing any part of a pen mate (= piglet or sow), including nose contact, without } \\
\text { manipulative behaviours or massaging the udder. }\end{array}$ \\
\hline \multicolumn{2}{|l|}{ Exploration } \\
\hline $\begin{array}{l}\text { Substrates- } \\
\text { directed } \\
\text { exploring }\end{array}$ & Sniffing, nosing, licking, rooting, rubbing substrates, or scraping the substrates with one leg. \\
\hline $\begin{array}{l}\text { Pen-directed } \\
\text { exploring }\end{array}$ & $\begin{array}{l}\text { Exploring any part of the pen (wall, floor), feeder, objects, drinking nipples and toys by sniffing, } \\
\text { nosing, licking, rooting, or rubbing. }\end{array}$ \\
\hline Chewing & \\
\hline $\begin{array}{l}\text { Substrates- } \\
\text { directed chewing }\end{array}$ & Chewing on substrates in enriched pens. \\
\hline $\begin{array}{l}\text { Pen-directed } \\
\text { chewing }\end{array}$ & $\begin{array}{l}\text { Chewing any part of the pen (wall, mat), feeder, objects, drinking nipples, toys or ear tags, or } \\
\text { chewing air or feces. }\end{array}$ \\
\hline Manipulation & $\begin{array}{l}\text { Nibbling, sucking, chewing, or biting an ear or the tail or other part of the body of a pen mate in a } \\
\text { continuous state. }\end{array}$ \\
\hline Play behaviour & $\begin{array}{l}\text { Shaking of head while holding substrate (e.g. straw, except toy) that protrudes from mouth, or } \\
\text { walking around the pen with substrate in mouth or lifting over substrates, shaking toy, or lifting } \\
\text { over/pushing toys, running, jumping, rolling, turning with other pigs or individually, sometimes with } \\
\text { gently nudging of pen mates. }\end{array}$ \\
\hline Aggression & $\begin{array}{l}\text { Horizontal or vertical knocking with the head or forward thrusting with the snout toward another pig } \\
\text { (single event or short series of events, not during a fight); Intense mutual/individual ramming or } \\
\text { pushing a pen mate with or without biting the pen mate (not the sow); Biting a pen mate, except ear } \\
\text { or tail, but not during a fight. }\end{array}$ \\
\hline Mounting & Standing on hind legs while having front legs on another pig's back (not the sow). \\
\hline $\begin{array}{l}\text { Comfort } \\
\text { behaviour }\end{array}$ & $\begin{array}{l}\text { Rubbing body against objects or pen mates, scratching body with hind leg, stretching (part of) body, } \\
\text { or shaking body. }\end{array}$ \\
\hline Other behaviour & $\begin{array}{l}\text { All other behaviours, including standing, sitting, walking without other behaviours described before, } \\
\text { and eating, drinking, defecating and urinating. }\end{array}$ \\
\hline
\end{tabular}




\subsubsection{Weight gain and feed intake}

Body weight of the pigs was measured at day 0 (within $12 \mathrm{~h}$ from birth), 28 (weaning), 33, and at day 46 (the day before the switch), 50 (3 days after the switch), 74 (27 days after the switch), 109 (62 days after the switch), and 130 (83 days after the switch). Feed intake was calculated per pen by registering the amount of feed given and weighing residual feed at several time points. Before weaning, residual feed was weighed at weaning on day 28. After weaning, residual feed was weighed on day 33, 47 (switch), 50 (3 days after the switch), and 133 (86 days after the switch).

\subsubsection{Statistical analyses}

SAS (SAS 9.4, SAS Institute Inc.) was used for all statistical analyses. Before weaning, 377 pigs were included in the home pen behaviour observation analyses. From the 192 pigs selected at weaning for further study, one pig died on the day of weaning (cause unknown), therefore, there were only 5 pigs in that pen from weaning onwards. Behaviour data from 3 other pigs were missing for the last observation day at 125 days of age, as they were euthanized due to health problems (lameness: $n=2$ and umbilical hernia: $n=1$ ). Pen-directed and substrate-directed exploring were combined as 'exploring'; pen-directed chewing and substrate-directed chewing were combined as 'chewing'. Pen-directed and substrate-directed exploring and chewing were also analysed separately. Comfort behaviour was rare (before weaning: $0.8 \%$, before the switch: $0.3 \%$, after the switch: $0.2 \%$ ) and therefore not further analysed. Defecating/urinating, eating, drinking, and walking, sitting and standing without any other behaviour were combined as 'other' behaviour, and were not further analysed as well. 
Assumptions of normality of error distribution and homogeneity of variance were checked, and proportions of time spent 2 days before the switch on aggression, pen-directed chewing, social behaviour and mounting were arcsine square root transformed. The same held for these behaviours and pen-directed exploration and play after the switch.

Behaviours

Before the switch. Proportions of time spent on behaviours before weaning and before the switch were analysed using a linear mixed model. The fixed effects of housing 1 ( $H 1$, housing before switch, B1 vs. E1), coping style (HR vs. LR), their interaction, sex and batch, and the random effect of pen nested with $\mathrm{H} 1$ and batch were included.

After the switch. Proportions of time spent on behaviours over the 5 observation days after the switch were analysed using a repeated linear mixed model with fixed effects of $\mathrm{H} 1$, housing $2(\mathrm{H} 2$, housing after switch, $\mathrm{B} 2$ vs. E2), coping style, observation day, their interactions, sex, the interaction of sex and observation day, and batch. Random effects of pen and animal were included to account for repeated measurements at the group (housing and batch) or individual level (sex and coping style).

Body weight gain

Before the switch. Birth weight and body weight gains from birth till weaning, and during the first 5 and 18 days after weaning of the 192 selected pigs were analysed using a linear mixed model, with $\mathrm{H} 1$, coping style, their interaction, sex, and batch as fixed effects, and pen nested with $\mathrm{H} 1$ and batch as random 
effect. Body weight at the beginning of each period was used as a covariate in the model, except for the analysis of body weight at birth.

After the switch. Body weight gains during several periods (day $50-46$, day 74 - 46, day 109 - 74, day 130 - 109, and day 130 - 46) after the switch and body weight on day 130 were analysed using a linear mixed model, with $\mathrm{H} 1$, $\mathrm{H} 2$, coping style, their interactions, sex, and batch as fixed effects, and pen nested with $\mathrm{H} 1, \mathrm{H} 2$, and batch as random effect. The body weight at the beginning of each period was used as a covariate in the model, except for the analysis of the body weight on day 130 .

Feed intake

Before weaning. Feed intake (measured at pen level, and expressed as $\mathrm{kg} /$ piglet) before weaning (days 5 - 28) was analysed using a linear model, with $\mathrm{H} 1$ and batch as fixed effects.

Before the switch. Average daily feed intake (kg/pig/day) of different periods (day 33 - 28 and day 47 - 28, i.e. the first five and 19 days after weaning, respectively) before the housing switch was analysed using a linear mixed model, with $\mathrm{H} 1$ and batch as fixed effects.

After the switch. Average daily feed intake of different periods (day 50 - 47 and day 133 - 47) after the housing switch was analysed using a linear mixed model, with $\mathrm{H} 1, \mathrm{H} 2$, their interaction, and batch as fixed effects.

Significant interactions were further investigated with post hoc pairwise comparisons using the difference of the least square means. Pairwise comparisons ( $>4$ groups of means) were adjusted by Tukey corrections. Results are presented as means \pm SEM. 


\subsection{Results}

\subsubsection{Behaviour observations}

\section{Before weaning}

Table 4.2 shows the percentage of time spent on different behaviours for barren (B1) vs. enriched (E1) housed pigs at 3 weeks of age, before weaning. E1 pigs tended to show less inactive behaviour $(p<0.10)$, and showed more exploring $(p<0.01)$ and chewing than B1 pigs $(p<0.001)$. Exploring and chewing of B1 pigs was pen-directed only, whereas E1 pigs could also use the substrates. The pen-directed exploring and chewing of E1 pigs was lower than that of $B 1$ pigs (both $p<0.001$ ). Moreover, E1 pigs showed less manipulative behaviours directed at pen mates $(p<0.001)$, less aggression $(p<0.05)$, and tended to show more play $(p<0.10)$. Housing did not affect social behaviour or mounting.

Table 4.2: Means \pm SEM of the percentages of time spent on behaviours in pigs housed in barren and enriched housing conditions at 3 weeks of age.

\begin{tabular}{llll}
\hline Behaviour (\% of time) & Barren & Enriched & p-value \\
\hline Inactive & $57.5 \pm 2.4$ & $51.9 \pm 2.0$ & + \\
Social behaviour & $1.8 \pm 0.2$ & $1.4 \pm 0.1$ & $\mathrm{~ns}$ \\
Exploring $^{1}$ & $7.2 \pm 0.6$ & $11.1 \pm 1.1$ & $* *$ \\
Pen-directed exploring $_{\text {Chewing }}^{2}$ & $7.2 \pm 0.6$ & $3.4 \pm 0.3$ & $* * *$ \\
Pen-directed chewing & $2.8 \pm 0.4$ & $10.0 \pm 1.1$ & $* * *$ \\
Manipulation & $2.8 \pm 0.4$ & $1.3 \pm 0.1$ & $* * *$ \\
Play & $2.4 \pm 0.2$ & $0.7 \pm 0.1$ & + \\
Aggression & $1.5 \pm 0.3$ & $2.4 \pm 0.4$ & $*$ \\
Mounting & $1.2 \pm 0.2$ & $0.7 \pm 0.1$ & $\mathrm{~ns}$ \\
\hline
\end{tabular}

${ }^{1}$ Exploration includes pen-directed exploring and exploring the substrates; ${ }^{2}$ chewing includes chewing the pen/air and chewing substrates. Significances of differences are indicated: ${ }^{* * *} p<0.001,{ }^{* *} p<0.01$, ${ }^{*} p<0.05$, and $+p<0.10 ; n s$ indicates non-significance. 
No coping style effects were found on the behaviour before weaning.

Females showed less aggressive behaviours (F: $0.6 \pm 0.1, \mathrm{M}: 1.3 \pm 0.1 \%$ of observations, $\mathrm{p}<0.001)$, and mounting ( $\mathrm{F}: 0.2 \pm 0.04, \mathrm{M}: 0.6 \pm 0.07 \%$, $p<0.001)$, and tended to chew more $(F: 7.0 \pm 0.4, M: 6.3 \pm 0.4 \%, p<0.10)$ before weaning than males. No other sex effects were found.

\section{Before the switch}

Table 4.3 shows the percentage of time spent on behaviours for enriched (E1) vs. barren (B1) housed pigs 2 days before the switch, at 6 weeks of age. E1 pigs displayed less inactive behaviour than B1 pigs $(p<0.001)$. Besides, total time spent on exploration was affected by $\mathrm{H} 1(\mathrm{E} 1>\mathrm{B} 1, \mathrm{p}<0.01)$, and tended to be affected by the $\mathrm{H} 1 \times$ coping style interaction $(\mathrm{p}<0.10)$. Pen-directed exploration was lower in E1 pigs than in B1 pigs $(p<0.001)$; and was also affected by coping style (HR: $4.0 \pm 0.4$, LR: $3.4 \pm 0.3 \%, p<0.05)$ and a trend for an interaction between $\mathrm{H} 1$ and coping style $(p<0.10)$. Chewing was affected by $\mathrm{H} 1$ (E1>B1, $\mathrm{p}<0.001)$, coping style (HR: $16.5 \pm 1.2 ; \mathrm{LR}: 14.9 \pm 1.0 \%$, $p<0.05)$ and tended to be affected by their interaction $(p<0.10)$. When looking at pen-directed chewing only, levels were lower for E1 pigs than for B1 pigs $(p<0.001)$. E1 pigs showed less manipulative behaviours directed at pen mates than B1 pigs $(p<0.001)$. Mounting was affected by $\mathrm{H} 1(\mathrm{E} 1<\mathrm{B} 1$, $p<0.05)$ and tended to be affected by coping style (HR: $0.7 \pm 0.1, L R: 0.6 \pm$ $0.1 \%, p<0.10)$. Housing or coping style did not affect social behaviour, play behaviour or aggression (see Table 4.3). 
Table 4.3: Means \pm SEM of the percentages of time spent on behaviours in pigs housed in barren and enriched housing conditions 2 days before the switch (at 47 days of age).

\begin{tabular}{llll}
\hline Behaviour (\% of time) & Barren & Enriched & p-value \\
\hline Inactive & $58.2 \pm 2.3$ & $44.7 \pm 2.0$ & $* * *$ \\
Social behaviour & $0.8 \pm 0.1$ & $0.7 \pm 0.1$ & $\mathrm{~ns}$ \\
Exploring $^{1}$ & $6.1 \pm 0.6$ & $8.8 \pm 0.6$ & $* *$ \\
Pen-directed exploring $_{\text {Chewing }}^{2}$ & $6.1 \pm 0.6$ & $1.4 \pm 0.1$ & $* * *$ \\
Pen-directed chewing & $7.6 \pm 0.7$ & $23.8 \pm 1.3$ & $* * *$ \\
Manipulation & $7.6 \pm 0.7$ & $0.8 \pm 0.1$ & $* * *$ \\
Play & $2.6 \pm 0.3$ & $0.6 \pm 0.2$ & $* * *$ \\
Aggression & $0.9 \pm 0.2$ & $1.4 \pm 0.2$ & $\mathrm{~ns}$ \\
Mounting & $0.7 \pm 0.1$ & $0.5 \pm 0.1$ & $\mathrm{~ns}$ \\
\hline
\end{tabular}

${ }^{1}$ Exploration includes exploring the pen and exploring the substrates; ${ }^{2}$ chewing includes chewing the pen/air and chewing substrates. Significances of differences are indicated: ${ }^{* *} p<0.001, * * p<0.01$, ${ }^{*} p<0.05$; ns indicates non-significance.

Females showed less aggression (F: $0.3 \pm 0.1, \mathrm{M}: 1.0 \pm 0.1 \%, \mathrm{p}<0.001$ ) and less mounting ( $\mathrm{F}: 0.4 \pm 0.1, \mathrm{M}: 1.0 \pm 0.1 \%, \mathrm{p}<0.001)$ than males, but chewed more (F: $16.8 \pm 1.2, M: 14.7 \pm 1.0 \%, p<0.01)$. No other sex effects were found.

\section{After the switch}

Figure 4.1 shows the time course of inactive, social, explorative, chewing, manipulative and play behaviour for the four combinations (B1B2, B1E2, E1B2, E1E2) of early life ( $\mathrm{H} 1$, housing before the switch) and later life $(\mathrm{H} 2$, housing after the switch) housing. As several interactions between housing and coping style were found, which were generally not dependent on time (no interactions with observation day, except for manipulation, see text below), these are illustrated separately in Figure 4.2. Aggression and mounting were mainly affected by sex and are therefore only indicated in the text. 

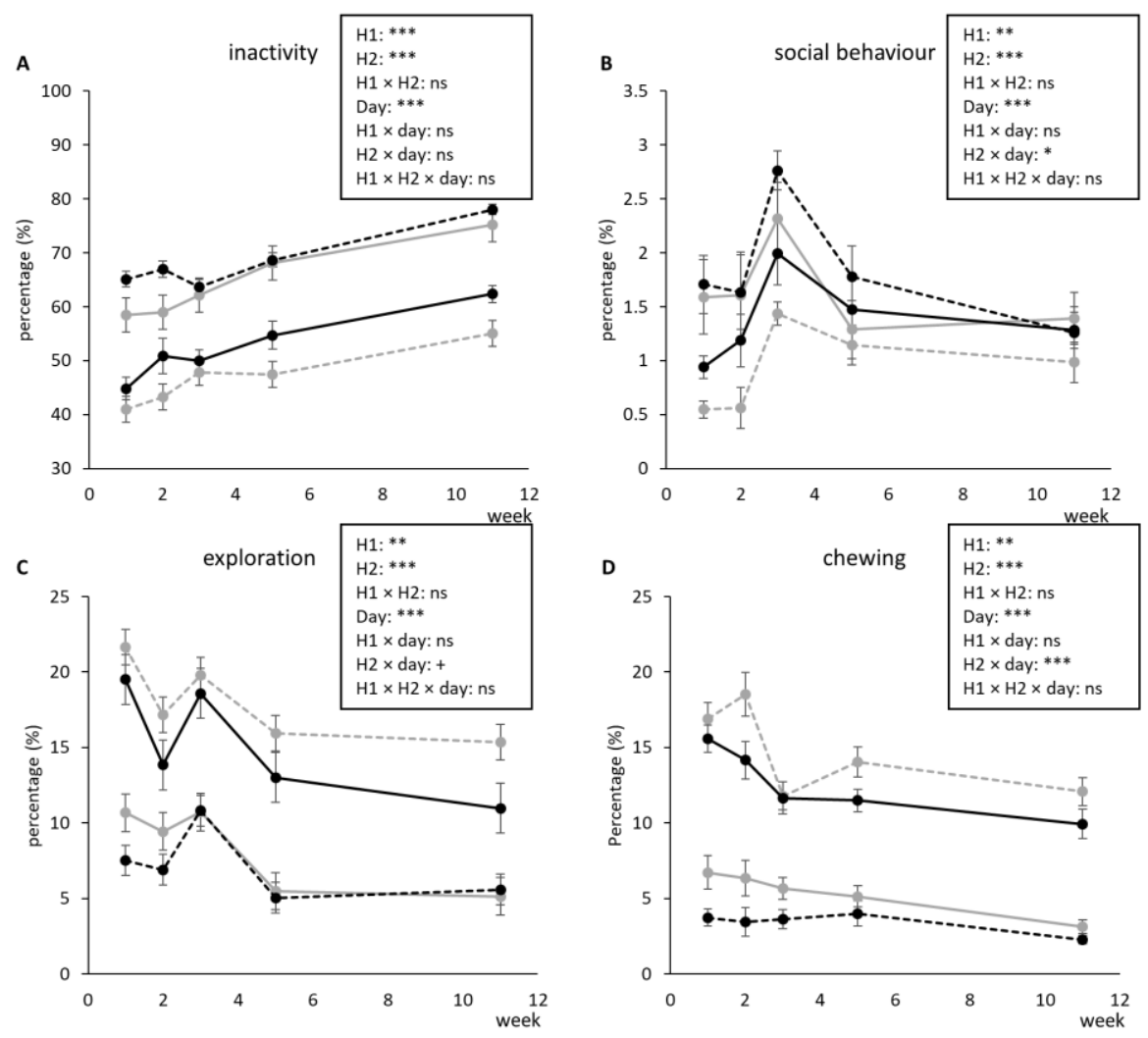

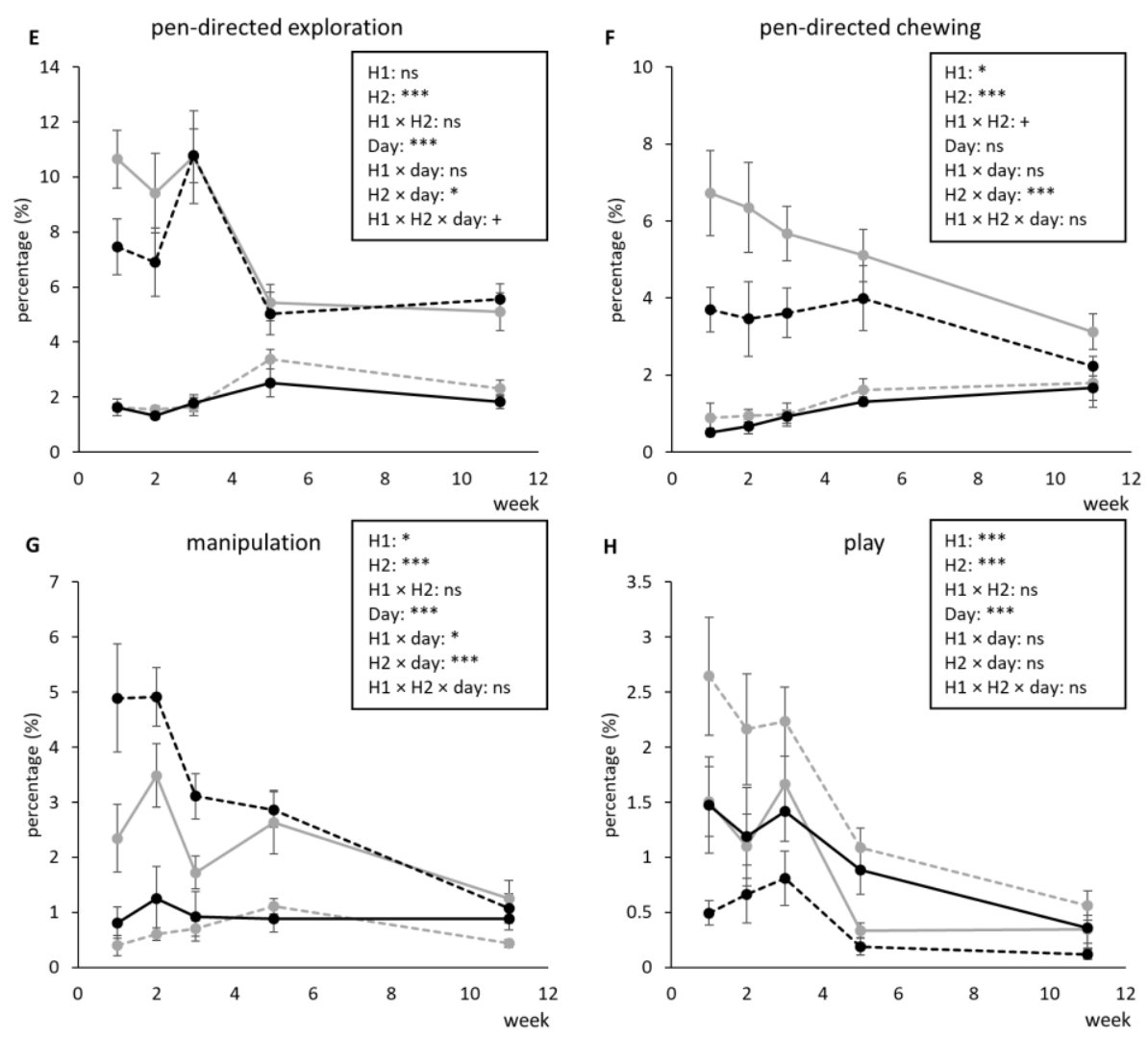

Figure 4.1: Means \pm SEM of percentages of time spent on behaviours in pigs exposed to four different housing situations ( $n=192$ pigs, 32 pens, 8 pens/housing treatment). Pigs were housed in either barren (B1) or enriched (E1) pens from birth, and half of them experienced a housing switch at 47 days of age, creating four groups: B1B2, B1E2, E1E2, and E1B2. Significances of treatments are indicated: $* * * p<0.001$, ${ }^{*} p<0.01, * p<0.05$, and $+p<0.10 ; n s$ indicates non-significance. H1 indicates the effect of housing before the switch (B1 vs. E1), and $\mathrm{H} 2$ indicates the effect of housing after the switch (B2 vs. E2). Interactions with coping style are given in Figure 2.

Inactive behaviour generally increased over time (day effect, $p<0.001$, Figure 4.1A). Pigs originating from enriched conditions (E1) were more inactive than 
those from early life barren housing ( $B 1, \mathrm{H} 1$ effect $p<0.001$ ). An opposite effect was found for $\mathrm{H} 2$ (E2 $<B 2, p<0.001)$, but the $\mathrm{H} 2$ effect depended on coping style ( $\mathrm{H} 2 \times$ coping style interaction, $\mathrm{p}<0.05)$, and tended to be affected by the $\mathrm{H} 1 \times \mathrm{H} 2 \times$ coping style interaction $(\mathrm{p}<0.10)$. Post hoc analysis of the $\mathrm{H} 2 \times$ coping style interaction showed that $\mathrm{B} 2-\mathrm{HR}$ pigs were the most inactive, followed by B2-LR pigs and thereafter all E2 pigs (B2-HR: $68.1 \pm 1.2$, B2-LR: $64.9 \pm 0.6$, E2-HR: $49.2 \pm 1.5$, E2-LR: $50.2 \pm 1.3 \%$, all pairwise differences mentioned $p<0.05$, Figure $4.2 \mathrm{~A}$ ).

Social behaviour was affected by day $(p<0.001), \mathrm{H} 2(p<0.001)$ and the $\mathrm{H} 2 \times$ day interaction $(p<0.05)$, with higher levels for B2 pigs than for E2 pigs on the first observation day ( 2 days after the switch) only (Figure 4.1B). Social behaviour was also affected by $\mathrm{H} 1(p<0.01)$, coping style $(p<0.10)$, and the $\mathrm{H} 2 \times$ coping style $(p<0.05)$ and $\mathrm{H} 1 \times \mathrm{H} 2 \times$ coping style $(p<0.05)$ interactions (Figure 4.2B). Levels of social behaviour were generally lowest in the pigs that switched from barren to enriched housing (B1E2) and highest in the E1B2 pigs, but the contrast with the other housing conditions depended on coping style. B1E2-HR pigs showed less social behaviour than E1E2-HR pigs $(p<0.05)$, with no other significant pairwise differences between housing conditions for the HR pigs. More contrasts between the $\mathrm{H} 1$ and $\mathrm{H} 2$ combinations were seen in LR animals. B1E2-LR showed lower levels of social behaviour than E1B2-LR $(p<0.001)$ and B1B1-LR pigs $(p<0.05)$. Additionally, E1B2-LR pigs, apart from showing more social behaviour than B1E2-LR pigs, also spent more time on this behaviour than E1E2-LR pigs, $p<0.05$, Figure 4.2B).

Exploration overall was affected by day $(p<0.001)$, with a general decrease in time, except for the third observation day (Figure 4.1C), and tended to be 
affected by the $\mathrm{H} 2 \times$ day interaction $(p<0.10) . \mathrm{H} 1$ (B1: $13.1 \pm 0.4, \mathrm{E} 1: 11.2 \pm$ $0.3 \%, p<0.01)$ and $H 2(B 2: 7.7 \pm 0.2, E 2: 16.6 \pm 0.3 \%, p<0.001)$ affected exploration in opposite directions. Besides, exploration tended to be affected by the $\mathrm{H} 2 \times$ coping style interaction ( $p<0.10$, Figure $4.2 \mathrm{C}$ ).

When analysing pen-directed exploration separately, effects of day $(p<0.001)$, $\mathrm{H} 2(p<0.001)$ and $\mathrm{H} 2 \mathrm{x}$ day $(\mathrm{p}<0.001)$ were found, as well as a trend for the $\mathrm{H} 1 \times \mathrm{H} 2 \times$ day interaction $(\mathrm{p}<0.10)$. For $\mathrm{E} 2$ pigs, time spent on pen-directed exploration did not differ between days. In B2 pigs, pen-directed exploration was higher on day 60 than on day 49 and day 54, and later in life, at 78 and 125 days of age, lower than the earlier three days (Figure 4.1E). In addition, coping style $(p<0.05)$ and the $H 2 \times$ coping style interaction $(p<0.05)$ affected pen-directed exploration, with a trend for the $\mathrm{H} 1 \times \mathrm{H} 2 \times$ coping style interaction ( $p<0.10$, Figure 4.2E). The two-way interaction between $\mathrm{H} 2$ and coping style showed that B2-LR pigs spent more time exploring pen fixtures than B2-HR pigs, which were, in turn, followed by E2 pigs (B2-HR: $7.2 \pm 0.3$, B2-LR: $8.2 \pm 0.3, E 2-H R: 1.9 \pm 0.1$, E2-LR: $2.0 \pm 0.1 \%, p<0.05)$.

When analysing substrate exploration separately (for the E2 pigs only, as B2 pigs did not have substrates), a day effect was found $(p<0.001)$, with a general decrease over time, except for day 60 (the third observation after housing switch). There was also a $\mathrm{H} 1$ effect showing that pigs originating from barren housing (B1E2: $15.8 \pm 0.4 \%$ ) spent more time on this behaviour than pigs that were housed in enriched conditions throughout (E1E2: $13.4 \pm$ $0.4 \%, p<0.05)$.

Chewing overall was affected by day $(p<0.001), \mathrm{H} 2(p<0.001)$, and the $\mathrm{H} 2 \times$ day interaction $(p<0.001$, Figure 4.1D). Time spent chewing did not change 
over time in B2 pigs, but in E2 pigs time spent chewing was higher on day 49 and 54 than on the days thereafter. Pigs from early life B1 pens showed more chewing $(10.0 \pm 0.3 \%)$ than those from E1 pens $(8.0 \pm 0.3 \%$, H1 effect, $p<0.05)$.

Pen-directed chewing was affected by $\mathrm{H} 2 \mathrm{x}$ day $(\mathrm{p}<0.001$, Figure $4.1 \mathrm{~F})$. It increased in E2 pigs (with higher levels on day 78 and 125 than on day 49), but decreased in B2 pigs (with lower levels on day 125 than on other days). Pen-directed chewing was also affected or tended to be affected by $\mathrm{H} 1$ $(p<0.05), H 2 \quad(p<0.001)$, their interaction $(H 1 \times H 2, p<0.10)$, and the interactions between $\mathrm{H} 1 \times$ coping style $(p<0.05), \mathrm{H} 2 \times$ coping style $(p<0.10)$, $\mathrm{H} 1 \times \mathrm{H} 2 \times$ coping style $(p<0.05)$ and $\mathrm{H} 1 \times \mathrm{H} 2 \times$ coping style $x$ day $(p<0.10)$. The three-way interaction ( $\mathrm{H} 1 \times \mathrm{H} 2 \times$ coping style) indicated that generally pigs from E2 housing chewed less on pen-fixtures than those from B2 housing, but in HR pigs, enriched housing in early life seemed to reduce this effect, as E1B2-HR pigs chewed less than E1B2-LR pigs and B1B2-HR pigs $(p<0.05$, Figure 4.2F).

When analysing substrate chewing separately for the pigs in enriched housing after the switch, a day effect $(p<0.001)$ was found, showing a decrease in time spent on this behaviour from day 60 onwards (course depending on sex, see below). Pigs originating from barren housing (B1E2: $13.5 \pm 0.4 \%)$ spent more time on chewing substrates than pigs that were housed in enriched conditions throughout (E1E2: $11.6 \pm 0.3 \%$, H1 effect, $p<0.05)$.

Manipulation was affected by $\mathrm{H} 1(\mathrm{p}<0.05), \mathrm{H} 2(\mathrm{p}<0.001), \mathrm{H} 2 \times$ coping style $(P<0.01)$ and $\mathrm{H} 1 \times \mathrm{H} 2 \times$ coping style $(p<0.01)$, day $(p<0.001), \mathrm{H} 1 \times$ day $(p<0.05)$, 
$\mathrm{H} 2 \mathrm{x}$ day $(\mathrm{p}<0.001)$ (Figure 4.1G), coping style $\mathrm{x}$ day $(\mathrm{p}<0.01), \mathrm{H} 1 \times$ coping style $x$ day $(p<0.05)$ and $\mathrm{H} 1 \times \mathrm{H} 2 \times$ coping style $x$ day $(p<0.05)$. Post-hoc analyses showed that within HR pigs, B2-HR pigs (both B1B2 and E1B2) manipulated more than E2-HR (B1E2 and E1E2) pigs $(p<0.05)$, whereas LR pigs that switched from enriched to barren housing (E1B2-LR) showed more manipulation than all others, including $H R$ pigs under the same circumstances (E1B2-HR) and LR pigs that were housed in barren pens throughout (B1B2-LR, $\mathrm{p}<0.05$, Figure 4.2G). On the last observation day (day 125), however, the $\mathrm{H} 1 \times \mathrm{H} 2 \times$ coping style interaction had disappeared as $\mathrm{H} 1$ and the interaction between housing and coping style did not influence time spent on oral manipulation on that day, whereas $\mathrm{H} 2$ and coping style effects remained.

Play decreased over time $(p<0.001)$, and was affected by $\mathrm{H} 1$ and $\mathrm{H} 2$ in opposite directions, as pigs from early life barren housing (B1: $1.4 \pm 0.1 \%$ ) played more than pigs originating from enriched pens before the switch (E1: $0.8 \pm 0.05 \%, p<0.001)$, whereas enrichment in later life increased play behaviour (B2: $0.7 \pm 0.05, E 2: 1.4 \pm 0.1 \%, p<0.001$ ).

Aggression generally decreased over time (day effect, $p<0.001$ ). In B2 pigs, aggressive behaviour was higher on day 54 (1.0 $\pm 0.1 \%)$ than on day 78 (0.5 $\pm 0.1 \%)$ and $125(0.4 \pm 0.1 \%)$, and in E2 pigs aggression was higher on day $49(1.1 \pm 0.1 \%)$ than on day $125(0.3 \pm 0.1 \%, \mathrm{H} 2 \mathrm{x}$ day effect, $\mathrm{p}<0.05)$, without a $\mathrm{H} 2$ effect within any of the days. Time spent on aggression was also affected by coping style (HR: $0.6 \pm 0.04, \mathrm{LR}: 0.8 \pm 0.04 \%, \mathrm{p}<0.01)$, and tended to be affected by the $\mathrm{H} 1 \times \mathrm{H} 2 \times$ coping style interaction $(\mathrm{p}<0.10)$. 
Mounting decreased over time (day effect $p<0.001$ ), being lower on the last two observation days than on the first observation day after the switch, but this only held for B2 pigs (day 49: $0.6 \pm 0.08$, day 78: $0.2 \pm 0.06$, day 125: 0.3 $\pm 0.06 \%)$, whereas time spent mounting was rather constant in E2 pigs ( $\mathrm{H} 2 \mathrm{x}$ day interaction, $p<0.05$, average: $0.3 \pm 0.03 \%$ ).

\section{Sex effects}

After the switch, inactive behaviour tended to be affected by sex $(p<0.10)$, and was affected by the sex $x$ day interaction $(p<0.001)$, with females being more inactive at 60 days of age than males ( $p<0.05$, Supplementary Table 1$)$. Females showed less social behaviour (F: $1.3 \pm 0.1, \mathrm{M}: 1.6 \pm 0.1 \%, \mathrm{p}<0.001$ ), explored less (F: $11.8 \pm 0.3, \mathrm{M}: 12.4 \pm 0.3 \%, \mathrm{p}<0.05$ ), and showed less substrate exploration ( $F: 14.0 \pm 0.4, \mathrm{M}: 15.2 \pm 0.4 \%, \mathrm{p}<0.05)$ than males. In both males and females, time spent chewing and chewing substrates decreased over time, but the time course of this decrease differed between the sexes (sex $x$ day interaction, both $p<0.05$ ), without sex differences within days (see Supplementary Table 4.1). Additionally, manipulation was affected by the sex $x$ day interaction $(p<0.05)$, without significant pairwise differences (Supplementary Table 4.1). Play was affected by sex $(p<0.05)$ and sex $\times$ day $(p<0.001)$. Females played less than males on day 60 ( $p<0.05$, Supplementary Table 4.1). Sex and sex $x$ day effects $(p<0.001)$ found for aggression indicated that females spent less time on aggressive behaviours than males on all observation days, except the last one (Supplementary Table 4.1). Males mounted more $(0.5 \pm 0.04 \%)$ than females $(0.1 \pm 0.02 \%, p<0.001)$. No effect of sex or its interaction with day was found on pen-directed exploration and pen-directed chewing. 

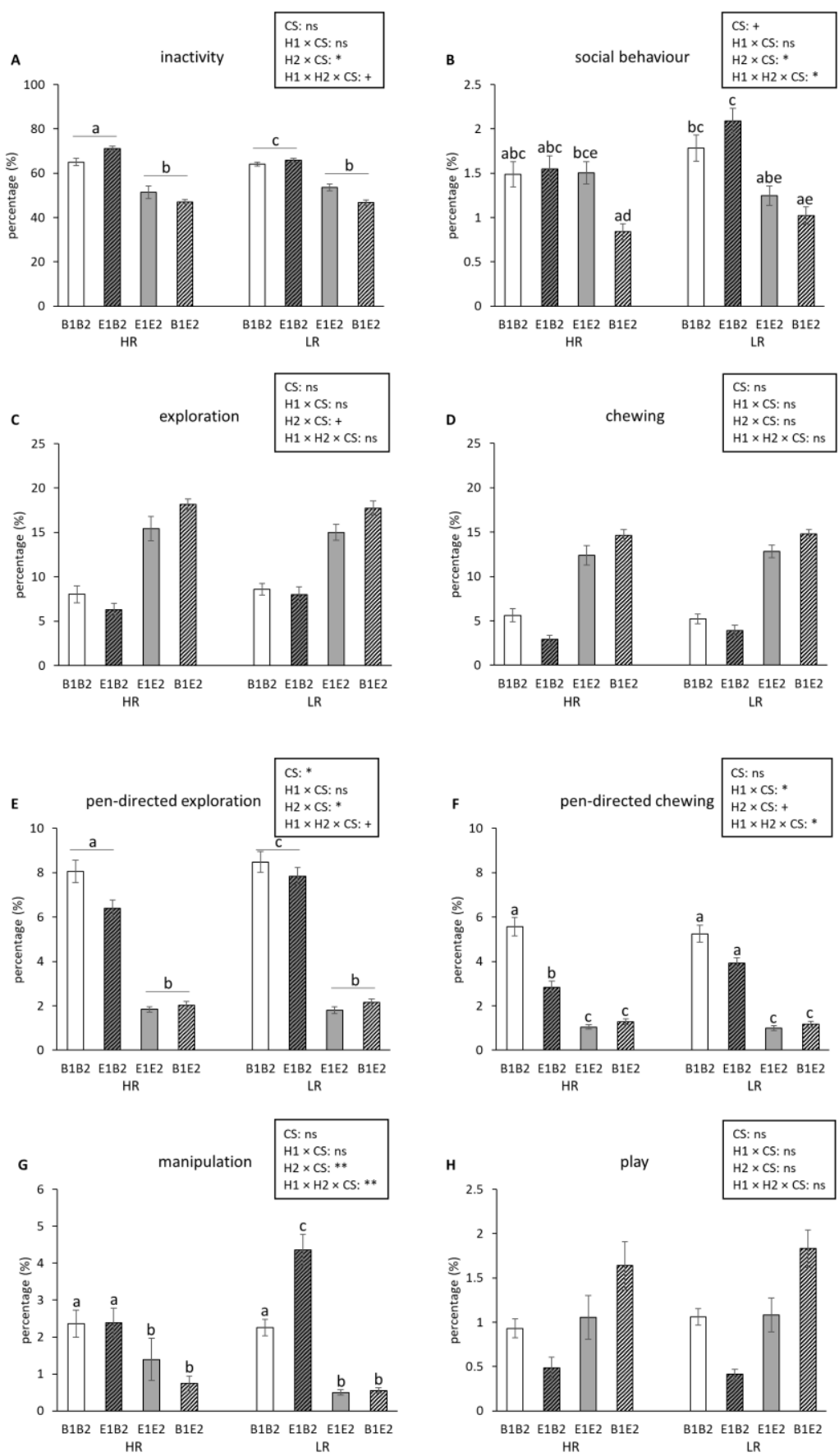
Figure 4.2: Means \pm SEM of percentages of time spent on behaviours for highresisters (HR) vs. low-resisters (LR) housed in four housing groups ( $n=192$ pigs, 32 pens, 8 pens/housing treatment) over 5 observation days after the switch. Means lacking a common letter differ significantly ( $p<0.05$ or less). Effects of coping style (CS) and its interactions with housing (housing before switch: $H 1$, housing after switch: H2) are indicated: ${ }^{* *} p<0.001,{ }^{* *} p<0.01,{ }^{*} p<0.05$, and $+p<0.1$; ns indicates nonsignificance. Please note: main effects of $\mathrm{H1}, \mathrm{H} 2$, their interaction and their interactions with day can be found in Figure 4.1.

\subsubsection{Body weight gain and feed intake}

Before the switch. The body weights of barren pigs and enriched pigs at birth were not different (B1: $1.5 \pm 0.03, \mathrm{E} 1: 1.5 \pm 0.02 \mathrm{~kg}$ ). From birth until weaning at day 28, E1 pigs gained more weight than B1 pigs (E1: $7.5 \pm 0.1$, B1: $6.9 \pm$ $0.2 \mathrm{~kg}, \mathrm{p}<0.05)$. Besides, LR pigs tended to gain more than HR pigs from birth till weaning (LR: $7.4 \pm 0.2 \mathrm{~kg}, H R: 7.0 \pm 0.2, p<0.10)$. Creep feed intake before weaning (day $5-28)$ tended to be higher in E2 pigs $(0.42 \pm 0.12 \mathrm{~kg} / \mathrm{pig})$ than in $B 2$ pigs $(0.30 \pm 0.09 \mathrm{~kg} / \mathrm{pig}, \mathrm{p}<0.10)$.

Enriched pigs also gained more weight during the first five (E1: $1.2 \pm 0.1, \mathrm{~B} 1$ : $0.8 \pm 0.1 \mathrm{~kg}, p<0.001$ ) and 18 days (E1: $9.1 \pm 0.3, B 1: 7.6 \pm 0.2 \mathrm{~kg}, p<0.001$ ) after weaning. Average daily feed intake during the first five (E1: $0.34 \pm 0.02$, B1: $0.28 \pm 0.02 \mathrm{~kg} / \mathrm{pig} /$ day, $\mathrm{p}<0.001$ ) and 19 days (E1: $0.61 \pm 0.01, \mathrm{~B} 1: 0.55 \pm$ $0.01 \mathrm{~kg} / \mathrm{pig} /$ day, $\mathrm{p}<0.01$ ) after weaning was also higher for E1 pigs than B1 pigs. As a result, E1 pigs were heavier $(18.1 \pm 0.3 \mathrm{~kg})$ just before the switch than $B 1$ pigs $(16.1 \pm 0.3 \mathrm{~kg}, \mathrm{p}<0.001)$.

After the switch. Table 4.4 shows the growth in the four housing treatment groups after the switch. With body weight at the start of each period as a covariate in the model, no significant housing effects were found for growth 
shortly after the switch (day $46-50$ ). Growth over the first 28 days after the switch (day $46-74$ ) tended to be higher in pigs in enriched housing after the switch (H2 effect, p<0.10). Growth between day 74 and 109 was not influenced by housing, but body weight gain from day 109 to day 130 was affected by $\mathrm{H} 1$ (E1: $22.6 \pm 0.6, \mathrm{~B} 1: 24.4 \pm 1.0 \mathrm{~kg}, \mathrm{p}<0.05)$, and H2 (E2: $24.4 \pm$ $1.0, B 2: 22.6 \pm 0.6 \mathrm{~kg}, \mathrm{p}<0.05)$ in opposite directions. Overall, the body weight gain from one day before the switch (day 46) until the end of the experiment (day 130) was higher for E2 $(86.0 \pm 1.0 \mathrm{~kg})$ than for B2 pigs $(81.4 \pm 0.9 \mathrm{~kg}, \mathrm{H} 2$ effect, $p<0.05)$. As a result, on day 130, E2 pigs were heavier $(103.4 \pm 1.0 \mathrm{~kg})$ than $\mathrm{B} 2$ pigs (98.2 $\pm 0.9 \mathrm{~kg}, \mathrm{p}<0.001)$.

Table 4.4: Means \pm SEM of body weight gains $(\mathrm{kg})$ in pigs housed in four housing groups after the housing switch (at 47 days of age).

\begin{tabular}{lllllll}
\hline Period & B1B2 & B1E2 & E1B2 & E1E2 & H1 & H2 \\
\hline day 46 - 50 & $2.5 \pm 0.2$ & $2.6 \pm 0.1$ & $2.6 \pm 0.3$ & $2.8 \pm 0.2$ & $\mathrm{~ns}$ & $\mathrm{~ns}$ \\
day 46 -74 & $20.5 \pm 0.8$ & $21.7 \pm 0.6$ & $22.1 \pm 0.9$ & $23.4 \pm 0.4$ & $\mathrm{~ns}$ & + \\
day 74 - 109 & $37.5 \pm 1.1$ & $38.8 \pm 1.6$ & $37.3 \pm 0.9$ & $39.4 \pm 1.3$ & $\mathrm{~ns}$ & $\mathrm{~ns}$ \\
day 109- 130 & $23.4 \pm 1.0$ & $25.4 \pm 1.8$ & $21.9 \pm 0.7$ & $23.4 \pm 1.0$ & $*$ & $*$ \\
day 46 - 130 & $81.5 \pm 1.3$ & $85.9 \pm 1.5$ & $81.3 \pm 1.2$ & $86.1 \pm 1.3$ & $\mathrm{~ns}$ & $* *$ \\
\hline
\end{tabular}

$\mathrm{H1}$ indicates the effect of housing before the switch, and $\mathrm{H} 2$ indicates the effect of housing after the switch. No $\mathrm{H} 1 \times \mathrm{H} 2$ interactions were found. Significances of differences are indicated: ${ }^{*} p<0.01$, ${ }^{*} p<0.05$, and $+p<0.10 ; n s$ indicates non-significance.

There were no coping style effects on body weight gain.

No sex effect was found on body weight gains before the housing switch. After the switch, females gained less weight than males in all periods, except the first days after the switch (data not shown). Their growth from the switch until day 130 therefore also diverged ( $F: 81.9 \pm 0.9, \mathrm{M}: 85.4 \pm 0.7 \mathrm{~kg}, \mathrm{p}<0.001)$ 
and at the end of the experiment on day 130 females were lighter than males (F: $98.9 \pm 1.0, M: 102.6 \pm 0.8 \mathrm{~kg}, \mathrm{p}<0.05)$.

During the first 3 days after the switch (day $47-50$ ), the average daily feed intake was affected by $\mathrm{H} 2$, with higher levels in $\mathrm{E} 2$ pigs $(1.6 \pm 0.2 \mathrm{~kg} / \mathrm{pig} / \mathrm{day})$ than in $\mathrm{B} 2$ pigs $(1.1 \pm 0.1 \mathrm{~kg} / \mathrm{pig} /$ day, $\mathrm{p}<0.05)$, but no $\mathrm{H} 1$ effect or $\mathrm{H} 1 \times \mathrm{H} 2$ interaction was found. In the period from the switch to the end of the experiment (day $47-133$ ), the average daily feed intake was also affected by $\mathrm{H} 2$ (E2: $2.1 \pm 0.04, \mathrm{~B} 2: 2.0 \pm 0.03, \mathrm{~kg} / \mathrm{pig} /$ day, $\mathrm{p}<0.01$ ) only.

\subsection{Discussion}

In this study, pigs were exposed to an either barren or enriched (with substrates, toys and more space) early life environment, after which half of them experienced a switch in housing conditions at 7 weeks of age. This allowed us to study the impact of early life and current housing, as well as that of an upgrade or downgrade shift in environmental conditions on their behaviour and body weight development up till 19 weeks of age. We found that the behaviour of the pigs after the switch reflected not only their actual environment, but was also influenced by early life housing conditions. Generally, effects of housing conditions after the switch seemed more pronounced in pigs that had experienced a different early life condition. Some of the (early life) housing effects depended on the coping style of the pigs.

\subsubsection{Effect of housing and coping style}


Effects of enrichment. Enrichment of the farrowing pen in the form of rooting substrates and extra toys and space reduced manipulative behaviour directed to pen mates, aggression, inactivity, increased exploration and chewing and tended to increase play behaviour before weaning. Similar results were found on day 45 ( 2 days before the housing switch), except that on this day barren and enriched pigs did not significantly differ in time spent on play and aggression anymore. The impact of an enriched environment reported here is largely in line with other studies using substrates and extra space (Beattie et al. 2000b; Beattie et al. 1995; Oostindjer et al. 2011) or substrates only (Bolhuis et al. 2005a; Bolhuis et al. 2006; Fraser et al. 1991) as enrichment. As expected, barren housed pigs spent more time on exploring their pen and on chewing on pen fittings or air, and performed more oral manipulative behaviour directed at their pen mates than enriched pigs. This confirms that pigs without suitable rooting materials direct more explorative behaviour to their pen and pen mates (Petersen et al. 1995). Nevertheless, total time spent on exploration and chewing were still lower for barren housed pigs, likely because the pen fittings and bare floor did not meet the criteria important to make exploration worthwhile, as pigs prefer 'chewable', 'destructible', 'rootable' and 'deformable' materials (Van de Weerd et al. 2003). It has been suggested that lowered levels of activity and exploration may reflect an apathetic response to an aversive environment and thus may indicate poor welfare (Fureix and Meagher 2015; Pearce et al. 1989; Wood-Gush and Beilharz 1983).

Enrichment reduced aggression before, but not after weaning. Beattie et al. (2000b) found a reduction in aggression in pigs provided with extra space and rooting substrates, whereas studies comparing similar sized pens with or 
without rooting substrates report either no effect (Bolhuis et al. 2005a; Fraser et al. 1991), a decrease (Beattie et al. 1996) or an increase in aggression (Mkwanazi et al. 2019; Morgan et al. 1998). Thus, effects of enrichment on aggression seem inconsistent.

Play behaviour is known to peak between 2 and 6 weeks of age in pigs and decreases thereafter with age (Newberry et al. 1988; Stolba and Wood-Gush 1989). Enriched housed pigs tended to spend more time on play behaviour before weaning and significantly played more after the switch in housing at 7 weeks. Also others reported more play behaviour in pigs kept in enriched as compared with barren pens (Bolhuis et al. 2005a; Bolhuis et al. 2006; Chaloupková et al. 2007a; Dudink et al. 2006). It has been put forward that several types of play behaviour are suppressed in adverse physical and environmental conditions (Ahloy-Dallaire et al. 2018) and following severe or prolonged negative emotions (Donaldson et al. 2002; Martin and Caro 1985). Some studies also suggest that a reduction in play behaviour in early life may negatively affect the development of behaviour and brain, leading to poorer social skills and behavioural flexibility, and therefore may have a long-term effect on adaptive capacity and welfare (Fagen 1982; Spinka et al. 2001; Van den Berg et al. 1999).

In this study, we found that enriched housed pigs were better able to cope with weaning transition, as they gained more weight and had a higher feed intake during the first five and 18 days after weaning. This could be related to the finding that enriched pigs also tended to eat more before weaning. Pre-weaning consumption of creep feed is known to stimulate post-weaning feed intake and thereby reduces the weaning-related growth check and 
other problems (e.g. Bruininx et al. 2002; Bruininx et al. 2004; Sulabo et al. 2010). The better post-weaning performance of enriched pigs may, apart from resulting from an enhanced development of feeding-related behaviours, also reflect increased adaptability of piglets reared in enriched conditions to stressful processes such as weaning (Oostindjer et al. 2010). However, it has been shown that post-weaning environmental enrichment alone, irrespective of pre-weaning housing, also improves performance and health of newly weaned piglets (Oostindjer et al. 2010), which may be mediated by preserving gut functioning (e.g. Pluske et al. 2007; Staals et al. 2007), either through intake of substrates or through stress reduction (e.g. Fraser 1985; Fraser et al. 1991).

After the housing switch. The behavioural development of the pigs from 7 to 19 weeks of age was characterized by an increase in inactivity, and a decrease in most of the active behaviours, in accordance with previous studies (e.g. Bolhuis et al. 2005a; Petersen et al. 1995). This is likely related to the increased age and weight and reduced space available per pig over time (Petersen et al. 1995). Effects of the current environment after 7 weeks were largely comparable to those before the switch. Pigs kept in enriched pens spent more time on exploration, chewing and play behaviour, whereas barren housed pigs were less active and showed more social behaviour, oral manipulation of pen mates and pen-directed exploration and chewing. The housing effect on social behaviour, which was not seen before 7 weeks of age, might partly reflect an increased inspection of pen mates' bodies in barren pens due to the absence of rooting substrates. 
Animals experiencing a switch in environment thus generally adjusted their behaviour to the current housing. Housing before the switch at 7 weeks of age, however, still exerted an influence on all behaviours with the exception of pen-directed exploration, demonstrating an interplay of past experiences and the current housing environment. Notably, for all behaviours except pendirected chewing, the effect of housing before the switch was opposite to that of housing after the switch. Adding up the early life and current housing effects, therefore, meant that the switched groups of pigs (E1B2 and B1E2) differed the most in their behaviour. For instance, both play and chewing were seen most often in pigs that switched from barren to enriched housing, and least in pigs experiencing a downshift from enriched to barren pens. Also, pigs that originated from barren housing spent more time on substraterelated behaviours after the switch than pigs kept in enriched housing throughout. Correspondingly, manipulative, social and inactive behaviour were highest in E1B2 pigs, and lowest in B1E2 pigs, albeit in a coping styledependent way (see below). Thus, a switch in housing conditions seemed to enlarge the effect of current housing on behaviour, implying a larger impact of presence or absence of enrichment in pigs with an opposite rearing history. It has been suggested that loss of enrichment, i.e. switching from enriched to barren housing, is more detrimental than barren housing throughout life (Latham and Mason 2010; Munsterhjelm et al. 2009), which seems to be proven in this study as well. Conversely, pigs that changed from barren to enriched housing seemed to show a 'catching-up' effect, which retained for months. This possibly reflects an increased motivation for exploration and play as the expression of these behaviours were hampered to lack of space 
and rooting materials earlier in life (Ruiterkamp 1985, cited in Bolhuis et al. 2005a).

The opposite effect of early life housing as compared with current housing, however, was not found for pen-directed chewing as B1B2 pigs spent more time on this behaviour than E1B2 pigs, although the effect was coping styledependent (see below). It could be that the E1B2 pigs, as they were used to more attractive substrates in early life, were not satisfied by chewing on penfixtures, and therefore turned to more nosing and oral manipulation of their pen mates, especially in the first weeks after the housing switch. It thus seems that there is no 'protective' effect of early enriched housing on the development of damaging behaviours, but rather a downshift ('frustration') effect was shown. Notably, early life housing had rather long-term effects on most of the behaviours, as there was no interaction between early life housing and day, except for manipulation, where the early life effect had disappeared by day 125 . Another study, however, found that the influence of early life rearing history on behaviour of pigs was smaller and merely overruled by current housing conditions (Bolhuis et al. 2006). The different results in the current study could be related to a larger contrast in housing, as enrichment here not only encompassed straw, but also other substrates and extra space, or to more observation weeks, and/or a larger sample size.

Even though we found long-term effects of the housing switch on behaviour, we can only speculate whether the interplay between early life and current housing also impacted the affective states of the pigs for such a long time. Douglas et al. (2012) reported more pessimistic judgement biases in pigs that had switched from enriched to barren pens as compared to pigs that initially 
were housed barren. However, in their study pigs were tested only shortly (up to 7 days) after the change in housing. We found that pigs experiencing an upgrade in the quality of their environment played most often, whereas pigs that were subjected to a downgrade from enriched to barren housing conditions showed the least play. Play behaviour has been linked to positive emotions (review in Held and Špinka 2011), and therefore, the findings in this study may suggest that a gain or loss of enrichment exerts a long-term impact on the affective state of pigs. However, other studies do not support this suggestion. We subjected part of the pigs to an attention bias test (Luo et al. 2019a) and successive negative contrast test (Luo et al. 2019b) to assess a potential long-term impact of their housing (history) on affective state, but failed to show the expected effects of the negative or positive housing switch on the responses of pigs in these tests. Rather, the successive negative contrast test suggested a 'protective' effect of early life enrichment irrespective of the current environment, as pigs reared in enriched pens were less susceptible to reward loss (Luo et al. 2019b). In the attention bias test no early life effects were found, whereas the impact of current housing was opposite to expectations, which might be due to the test circumstances (Luo et al. 2019a), and only found for pigs with a proactive coping style. Thus, further research is needed to evaluate whether a change from enriched to barren housing conditions and vice versa not only affects pigs' behaviour, but also their affective state in the long run.

Coping style effects. Effects of enrichment on several behaviours were found to be coping style-dependent, even though we did not select extremes for our study, but labelled each pig as either LR (reactive) or HR (proactive) based on a single backtest early in life. Barren housing increased inactivity in both 
types of pig, but more so in the HR pigs, whereas pen-directed exploration was enhanced in the barren housed LR pigs. Also, the impact of a switch in housing conditions on some behaviours varied for both types of pig, as social behaviour, pen-directed chewing and manipulation were affected by the interaction between early life housing, current housing and coping style. LR pigs that experienced a downgrade in housing from enriched to barren pens showed more pen-directed chewing and oral manipulation of pen mates than their HR counterparts. The strong increase in these oral damaging behaviours in LR pigs that had switched from enriched to barren housing, exceeding the levels of pigs kept in barren pens throughout, might indicate that LR pigs were most affected by the loss of exploratory stimuli and space. Also in other studies LR pigs showed more oral manipulation than HR pigs, especially when housed barren (Bolhuis et al. 2005a), and, in contrast to the current study, even more so when also reared in barren pens (Bolhuis et al. 2006). It has been suggested that LR pigs have a higher motivation to explore their surroundings than HR pigs (Jansen et al. 2009), and therefore show more manipulative behaviour directed at pen and pen mates when substrates are not available (Bolhuis et al. 2005a). The effects of rooting substrates on the occurrence of gastric lesions (Bolhuis et al. 2006), immune reactivity (Bolhuis et al. 2003) and response to novelty (Bolhuis et al. 2004) were also larger in LR than in HR pigs, suggesting that reactive pigs are more susceptible to (lack of) enrichment. In support of this, Asher et al. (2016) found that reactive pigs in barren pens were more pessimistic and those in enriched pens were more optimistic in a cognitive bias test, whereas the response of proactive pigs was less affected by their housing environment. It should be noted, though, that the housing effect on the behavioural 
response in an attention bias test was stronger for proactive pigs(Luo et al. 2019a), which is not in line with other findings.

Irrespective of housing treatments, LR pigs spent more time on aggressive behaviours than HR pigs after the housing switch. This is in contrast with other studies describing higher levels of aggression in HR pigs, both in stable groups (Bolhuis et al. 2005a; Bolhuis et al. 2006) and when mixed (Ruis et al. 2000), or reporting no differences between LR and HR pigs (D'Eath and Burn 2002). These inconsistent results could be related to the finding that, rather differing in level of aggression per se, the types of pig may vary in how flexibly they can attune their aggressive behaviour (Bolhuis et al. 2005b; Melotti et al. 2011).

Body weight gain. In the present study, enriched housed pigs had higher body weight gains than barren housed pigs, both before and after the switch, as well as a higher feed intake. At the end of the experiment (around 19 weeks of age), pigs kept in enriched pens from the switch at 7 weeks onwards were heavier, irrespective of their early life housing conditions. This means that pigs originating from barren housing, even though they had a lower body weight before the switch than enriched pigs, caught up after being placed in enriched pens. The reverse was found in pigs from enriched housing that changed to barren pens. This is illustrated by the growth from day 109 to day 130 , which was affected by both early life and current housing, but in opposite directions. As a result, B1E2 pigs gained the most weight, and E1B2 the least. Also other studies report that environmental enrichment can improve the growth of pigs (e.g. Beattie et al. 2000b; Lebret 2008). It has been shown that oral manipulation can negatively affect growth (Camerlink 
et al. 2012), which could be either due to stress in the victims, or because the wounds caused by oral manipulation may lead to inflammation and infection (Sihvo et al. 2012). In our study, barren pigs indeed showed more tail lesions following oral manipulation. It should, however, be noted that some other studies showed no difference in growth between enriched and barren housed pigs (Beattie et al. 1995; Morrison et al. 2007; Pearce et al. 1989). Additionally, feed intake was also higher in the enriched pigs, which is in line with other studies (Beattie et al. 2000b; Morrison et al. 2007; Van de Weerd et al. 2006). This could be related to the stimulation of exploration and/or easier access of pigs to the feeder in the enriched pens (Lebret et al. 2006), which can facilitate feeder visits and, consequently, may increase feed intake (Casal-Plana et al. 2017), and therefore increase body weight gains.

\subsubsection{Effects of sex}

In this study, males had higher body weight gains than females after the housing switch, which is in line with other studies (Campbell et al. 1985; Fuller et al. 1995; Latorre et al. 2003). In addition, males showed more mounting behaviour. Mounting could be an extension of aggression, but likely it is also a sexual behaviour here (Ford 1990). Stress can also negatively influence growth (Hemsworth et al. 1987; White et al. 2008). Females were probably more often victims of mounting than males (Clark and D'Eath 2013; Colson et al. 2006; Kaminder 1991; Rydhmer et al. 2006) leading to stress in the females, and consequently a lower weight gain. Males were found to display more aggressive behaviours than females in this study as well, which is in line with other studies (Clark and D'Eath 2013; Colson et al. 2006; Rydhmer et al. 2006), except on the last observation day when all pigs were 
getting less active. Furthermore, males displayed more social behaviour and exploration than females. The effect of the interaction between sex and day on inactivity, manipulation, chewing, and play revealed a different course of time spent on these behaviours over age, without clear sex effects on particular days.

\subsection{Conclusion}

Enrichment with rooting substrates and extra space profoundly affected behaviour and growth of pigs. The behaviour of pigs that switched from barren to enriched pens or vice versa reflected, however, not only their actual environment, but was also influenced by their early life housing conditions. Generally, effects of (lack of) enrichment after the switch seemed more pronounced in pigs that had experienced a different early life condition, with pigs undergoing a downgrade displaying signs of frustration, whereas pigs exposed to an upgrade seemed to 'catch up' by showing more exploration and play. Several effects of early life and current housing were stronger in pigs with a reactive personality. Thus, not only current housing conditions, but also a (mis)match with the early life environment may affect behaviour and growth of pigs.

\subsection{Acknowledgement}

The authors would like to thank M. van Marwijk, B. Laurenssen, and M. Ooms for skilful assistance in conducting the experiment. We are also grateful to 
the animal caretakers and students involved. The authors also appreciate the sponsor for Lu Luo from China Scholarship Council. 


\subsection{References}

Ahloy-Dallaire J, Espinosa J, Mason G (2018) Play and optimal welfare: Does play indicate the presence of positive affective states? Behavioural processes 156:3-15

Asher L, Friel M, Griffin K, Collins LM (2016) Mood and personality interact to determine cognitive biases in pigs Biology letters 12:20160402

Averós X, Brossard L, Dourmad J-Y, de Greef KH, Edge HL, Edwards SA, Meunier-Salaün M-C (2010) A meta-analysis of the combined effect of housing and environmental enrichment characteristics on the behaviour and performance of pigs Appl Anim Behav Sci 127:73-85

Beattie V, O'Connell N, Kilpatrick D, Moss B (2000a) Influence of environmental enrichment on welfare-related behavioural and physiological parameters in growing pigs Animal Science 70:443-450

Beattie V, O'connell N, Moss B (2000b) Influence of environmental enrichment on the behaviour, performance and meat quality of domestic pigs Livestock production science 65:71-79

Beattie V, Walker N, Sneddon I (1995) Effects of environmental enrichment on behaviour and productivity of growing pigs Animal Welfare 4:207220

Beattie V, Walker N, Sneddon I (1996) An investigation of the effect of environmental enrichment and space allowance on the behaviour and production of growing pigs Applied animal behaviour science 48:151-158

$B \varnothing e K(1993)$ The effect of age at weaning and post-weaning environment on the behaviour of pigs Acta Agriculturae Scandinavica A-Animal Sciences 43:173-180

Bolhuis J, Schouten W, De Jong I, Schrama J, Cools A, Wiegant V (2000) Responses to apomorphine of pigs with different coping characteristics Psychopharmacology 152:24-30

Bolhuis JE, Parmentier HK, Schouten WG, Schrama JW, Wiegant VM (2003) Effects of housing and individual coping characteristics on immune responses of pigs Physiology \& behavior 79:289-296

Bolhuis JE, Schouten WG, de Leeuw JA, Schrama JW, Wiegant VM (2004) Individual coping characteristics, rearing conditions and behavioural flexibility in pigs Behavioural Brain Research 152:351-360

Bolhuis JE, Schouten WG, Schrama JW, Wiegant VM (2005a) Behavioural development of pigs with different coping characteristics in barren 
and substrate-enriched housing conditions Applied Animal Behaviour Science 93:213-228

Bolhuis JE, Schouten WG, Schrama JW, Wiegant VM (2005b) Individual coping characteristics, aggressiveness and fighting strategies in pigs Animal Behaviour 69:1085-1091

Bolhuis JE, Schouten WG, Schrama JW, Wiegant VM (2006) Effects of rearing and housing environment on behaviour and performance of pigs with different coping characteristics Applied Animal Behaviour Science 101:68-85

Bruininx E, Binnendijk G, Van der Peet-Schwering C, Schrama J, Den Hartog L, Everts H, Beynen A (2002) Effect of creep feed consumption on individual feed intake characteristics and performance of grouphoused weanling pigs Journal of animal science 80:1413-1418

Bruininx E et al. (2004) Individually assessed creep food consumption by suckled piglets: Influence on post-weaning food intake characteristics and indicators of gut structure and hind-gut fermentation Animal Science 78:67-75

Camerlink I, Bijma P, Kemp B, Bolhuis JE (2012) Relationship between growth rate and oral manipulation, social nosing, and aggression in finishing pigs Applied Animal Behaviour Science 142:11-17

Camerlink I, Bolhuis J, Duijvesteijn N, Van Arendonk J, Bijma P (2014) Growth performance and carcass traits in pigs selected for indirect genetic effects on growth rate in two environments Journal of Animal Science 92:2612-2619

Campbell R, Taverner M, Curic D (1985) Effects of sex and energy intake between 48 and $90 \mathrm{~kg}$ live weight on protein deposition in growing pigs Animal Science 40:497-503

Carreras $\mathrm{R}$ et al. (2016) Housing conditions do not alter cognitive bias but affect serum cortisol, qualitative behaviour assessment and wounds on the carcass in pigs Applied Animal Behaviour Science 185:39-44

Casal-Plana N, Manteca X, Dalmau A, Fàbrega E (2017) Influence of enrichment material and herbal compounds in the behaviour and performance of growing pigs Applied Animal Behaviour Science 195:38-43

Chaloupková H, Illmann G, Bartoš L, Špinka M (2007a) The effect of preweaning housing on the play and agonistic behaviour of domestic pigs Appl Anim Behav Sci 103:25-34

Chaloupková H, Illmann G, Neuhauserová K, Tománek M, Valis L (2007b) Preweaning housing effects on behavior and physiological measures 
in pigs during the suckling and fattening periods Journal of animal science 85:1741-1749

Clark CC, D'Eath RB (2013) Age over experience: Consistency of aggression and mounting behaviour in male and female pigs Appl Anim Behav Sci 147:81-93

Colson V, Orgeur P, Courboulay V, Dantec S, Foury A, Mormède P (2006) Grouping piglets by sex at weaning reduces aggressive behaviour Appl Anim Behav Sci 97:152-171

D'Eath R, Burn C (2002) Individual differences in behaviour: A test of'coping style'does not predict resident-intruder aggressiveness in pigs Behaviour 139:1175-1194

Day J, Burfoot A, Docking C, Whittaker X, Spoolder H, Edwards S (2002) The effects of prior experience of straw and the level of straw provision on the behaviour of growing pigs Applied Animal Behaviour Science 76:189-202

de Jong IC et al. (1998) Effects of strawbedding on physiological responses to stressors and behavior in growing pigs Physiology \& behavior 64:303310

De Jonge FH, Bokkers $\mathrm{E}$, Schouten W, Helmond F (1996) Rearing piglets in a poor environment: developmental aspects of social stress in pigs Physiology \& Behavior 60:389-396

do Prado CH, Narahari T, Holland FH, Lee HN, Murthy SK, Brenhouse HC (2016) Effects of early adolescent environmental enrichment on cognitive dysfunction, prefrontal cortex development, and inflammatory cytokines after early life stress Developmental psychobiology 58:482-491

Donaldson TM, Newberry RC, Špinka M, Cloutier S (2002) Effects of early play experience on play behaviour of piglets after weaning Applied Animal Behaviour Science 79:221-231

Dudink S, Simonse H, Marks I, de Jonge FH, Spruijt BM (2006) Announcing the arrival of enrichment increases play behaviour and reduces weaningstress-induced behaviours of piglets directly after weaning Applied Animal Behaviour Science 101:86-101

Fagen R (1982) Evolutionary issues in development of behavioral flexibility. In: Ontogeny. Springer, pp 365-383

Ford J (1990) Differentiation of sexual behaviour in pigs Journal of reproduction and fertility Supplement 40:311-321 
Fraser D (1985) Selection of bedded and unbedded areas by pigs in relation to environmental temperature and behaviour Appl Anim Behav Sci 14:117-126

Fraser D, Phillips P, Thompson B, Tennessen T (1991) Effect of straw on the behaviour of growing pigs Applied Animal Behaviour Science 30:307318

Fuller M, Franklin M, McWilliam R, Pennie K (1995) The responses of growing pigs, of different sex and genotype, to dietary energy and protein Animal Science 60:291-298

Fureix C, Meagher RK (2015) What can inactivity (in its various forms) reveal about affective states in non-human animals? A review Applied Animal Behaviour Science 171:8-24

Hadley C, Hadley B, Ephraim S, Yang M, Lewis MH (2006) Spontaneous stereotypy and environmental enrichment in deer mice (Peromyscus maniculatus): reversibility of experience Applied Animal Behaviour Science 97:312-322

Held SD, Špinka M (2011) Animal play and animal welfare Animal behaviour 81:891-899

Hemsworth P, Barnett J, Hansen C (1987) The influence of inconsistent handling by humans on the behaviour, growth and corticosteroids of young pigs Appl Anim Behav Sci 17:245-252

Jansen J, Bolhuis JE, Schouten WG, Spruijt BM, Wiegant VM (2009) Spatial learning in pigs: effects of environmental enrichment and individual characteristics on behaviour and performance Animal cognition 12:303-315

Kallio P, Janczak A, Valros A, Edwards S, Heinonen M (2018) Case control study on environmental, nutritional and management-based risk factors for tail-biting in long-tailed pigs Animal Welfare

Kaminder M (1991) Relationship between skatole and agonistic or sexual behaviour in entire male pigs Examensarbete-Sveriges Lantbruksuniversitet

Koolhaas J et al. (1999) Coping styles in animals: current status in behavior and stress-physiology Neuroscience \& Biobehavioral Reviews 23:925-935

Koolhaas J, Van Reenen C (2016) Animal behavior and well-being symposium: Interaction between coping style/personality, stress, and welfare: Relevance for domestic farm animals Journal of animal science 94:2284-2296 
Kuzumaki N et al. (2011) Hippocampal epigenetic modification at the brainderived neurotrophic factor gene induced by an enriched environment Hippocampus 21:127-132

Lammers G, Schouten W (1985) Effect of pen size on the development of agonistic behaviour in piglets Netherlands journal of agricultural science 33:305-305

Landis JR, Koch GG (1977) The measurement of observer agreement for categorical data biometrics:159-174

Latham N, Mason G (2010) Frustration and perseveration in stereotypic captive animals: is a taste of enrichment worse than none at all? Behavioural Brain Research 211:96-104

Latorre M, Lázaro R, Gracia M, Nieto M, Mateos G (2003) Effect of sex and terminal sire genotype on performance, carcass characteristics, and meat quality of pigs slaughtered at $117 \mathrm{~kg}$ body weight Meat science 65:1369-1377

Lebret B (2008) Effects of feeding and rearing systems on growth, carcass composition and meat quality in pigs Animal 2:1548-1558

Lebret $B$, Meunier-Salaun $M$, Foury $A$, Mormède $P$, Dransfield $E$, Dourmad J (2006) Influence of rearing conditions on performance, behavioral, and physiological responses of pigs to preslaughter handling, carcass traits, and meat quality Journal of Animal Science 84:2436-2447

Lukkes JL, Mokin MV, Scholl JL, Forster GL (2009) Adult rats exposed to earlylife social isolation exhibit increased anxiety and conditioned fear behavior, and altered hormonal stress responses Hormones and behavior 55:248-256

Luo L, Dixhoorn IDE, Reimert I, Kemp B, Bolhuis JE, Parmentier HK (2017a) Effect of enriched housing on levels of natural (auto-) antibodies in pigs co-infected with porcine reproductive and respiratory syndrome virus (PRRSV) and Actinobacillus pleuropneumoniae Veterinary research 48:75

Luo L, Geers R, Reimert I, Kemp B, Parmentier H, Bolhuis J (2017b) Effects of environmental enrichment and regrouping on natural autoantibodies-binding danger and neural antigens in healthy pigs with different individual characteristics animal:1-8

Luo L, Reimert I, de Haas EN, Kemp B, Bolhuis JE (2019a) Effects of early and later life environmental enrichment and personality on attention bias in pigs (Sus scrofa domesticus) Animal Cognition:1-14 
Luo L, Reimert I, Graat E, Smeets S, Kemp B, Bolhuis J (2019b) Effects of early life and current housing on sensitivity to reward loss in a successive negative contrast test in pigs Animal cognition:1-10

Martin P, Bateson P (2007) Recording methods. In 'Measuring Behaviour: An Introductory Guide'. Cambridge University Press: Cambridge, UK,

Martin P, Caro TM (1985) On the functions of play and its role in behavioral development. In: Advances in the Study of Behavior, vol 15. Elsevier, pp 59-103

Melotti L, Oostindjer M, Bolhuis JE, Held S, Mendl M (2011) Coping personality type and environmental enrichment affect aggression at weaning in pigs Applied Animal Behaviour Science 133:144-153

Mkwanazi MV, Ncobela CN, Kanengoni AT, Chimonyo M (2019) Effects of environmental enrichment on behaviour, physiology and performance of pigs - A review Asian-Australasian journal of animal sciences 32:1

Moinard C, Mendl M, Nicol C, Green L (2003) A case control study of on-farm risk factors for tail biting in pigs Applied Animal Behaviour Science 81:333-355

Mora F, Segovia G, del Arco A (2007) Aging, plasticity and environmental enrichment: structural changes and neurotransmitter dynamics in several areas of the brain Brain research reviews 55:78-88

Morgan C, Deans L, Lawrence A, Nielsen BL (1998) The effects of straw bedding on the feeding and social behaviour of growing pigs fed by means of single-space feeders Applied Animal Behaviour Science 58:23-33

Morrison RS, Johnston LJ, Hilbrands AM (2007) The behaviour, welfare, growth performance and meat quality of pigs housed in a deep-litter, large group housing system compared to a conventional confinement system Appl Anim Behav Sci 103:12-24

Munsterhjelm C, Peltoniemi OA, Heinonen M, Hälli O, Karhapää M, Valros A (2009) Experience of moderate bedding affects behaviour of growing pigs Applied Animal Behaviour Science 118:42-53

Newberry R, Wood-Gush D, Hall J (1988) Playful behaviour of piglets Behavioural Processes 17:205-216

Oostindjer M, Bolhuis J, Mendl M, Held S, Gerrits W, Van den Brand H, Kemp $B$ (2010) Effects of environmental enrichment and loose housing of lactating sows on piglet performance before and after weaning Journal of Animal Science 88:3554-3562 
Oostindjer M, van den Brand H, Kemp B, Bolhuis JE (2011) Effects of environmental enrichment and loose housing of lactating sows on piglet behaviour before and after weaning Appl Anim Behav Sci 134:31-41

Pearce G, Paterson A, Pearce A (1989) The influence of pleasant and unpleasant handling and the provision of toys on the growth and behaviour of male pigs Appl Anim Behav Sci 23:27-37

Petersen V, Simonsen HB, Lawson LG (1995) The effect of environmental stimulation on the development of behaviour in pigs Applied Animal Behaviour Science 45:215-224

Pluske J, Durmic Z, Payne H, Mansfield J, Mullan B, Hampson D, Vercoe P (2007) Microbial diversity in the large intestine of pigs born and reared in different environments Livestock science 108:113-116

Powell SB, Newman HA, McDonald TA, Bugenhagen P, Lewis MH (2000) Development of spontaneous stereotyped behavior in deer mice: effects of early and late exposure to a more complex environment Developmental Psychobiology: The Journal of the International Society for Developmental Psychobiology 37:100-108

Reimert I, Rodenburg TB, Ursinus WW, Kemp B, Bolhuis JE (2014) Selection based on indirect genetic effects for growth, environmental enrichment and coping style affect the immune status of pigs PloS one 9:e108700

Ruis MA, te Brake JH, van de Burgwal JA, de Jong IC, Blokhuis HJ, Koolhaas JM (2000) Personalities in female domesticated pigs: behavioural and physiological indications Applied Animal Behaviour Science 66:31-47

Ruiterkamp WA (1985) Het gedrag van mestvarkens in relatie tot huisvesting. Rydhmer L, Zamaratskaia G, Andersson H, Algers B, Guillemet R, Lundström $K$ (2006) Aggressive and sexual behaviour of growing and finishing pigs reared in groups, without castration Acta Agriculturae Scand Section A 56:109-119

Scollo A, Contiero B, Gottardo F (2016) Frequency of tail lesions and risk factors for tail biting in heavy pig production from weaning to $170 \mathrm{~kg}$ live weight The Veterinary Journal 207:92-98

Sihvo H-K, Simola O, Munsterhjelm C, Syrjä P (2012) Systemic spread of infection in tail-bitten pigs Journal of Comparative Pathology 1:73

Simonsen HB (1995) Effect of early rearing environment and tail docking on later behaviour and production in fattening pigs Acta Agriculturae Scandinavica A-Animal Sciences 45:139-144 
Spinka M, Newberry RC, Bekoff M (2001) Mammalian play: training for the unexpected The quarterly review of biology 76:141-168

Staals S, Bolhuis J, Van den Brand H, Gerrits W (2007) Contribution of a straw bedding to digestible nutrient intake of pigs fed diets based on either native or pregelatinized potato starch Livestock Science 109:104-107

Stolba A, Wood-Gush D (1989) The behaviour of pigs in a semi-natural environment Animal Science 48:419-425

Sulabo R, Jacela J, Tokach M, Dritz S, Goodband R, DeRouchey JM, Nelssen J (2010) Effects of lactation feed intake and creep feeding on sow and piglet performance Journal of animal science 88:3145-3153

Taylor NR, Parker RM, Mendl M, Edwards SA, Main DC (2012) Prevalence of risk factors for tail biting on commercial farms and intervention strategies The Veterinary Journal 194:77-83

Telkänranta H, Bracke MB, Valros A (2014) Fresh wood reduces tail and ear biting and increases exploratory behaviour in finishing pigs Applied Animal Behaviour Science 161:51-59

Ursinus WW, Van Reenen CG, Kemp B, Bolhuis JE (2014) Tail biting behaviour and tail damage in pigs and the relationship with general behaviour: Predicting the inevitable? Applied Animal Behaviour Science 156:2236

Van de Weerd H, Docking C, Day J, Edwards S (2005) The development of harmful social behaviour in pigs with intact tails and different enrichment backgrounds in two housing systems Animal Science 80:289-298

Van de Weerd HA, Docking CM, Day JE, Avery PJ, Edwards SA (2003) A systematic approach towards developing environmental enrichment for pigs Applied Animal Behaviour Science 84:101-118

Van de Weerd HA, Docking CM, Day JE, Breuer K, Edwards SA (2006) Effects of species-relevant environmental enrichment on the behaviour and productivity of finishing pigs Applied Animal Behaviour Science 99:230-247

Van den Berg CL, Hol T, Van Ree JM, Spruijt BM, Everts H, Koolhaas JM (1999) Play is indispensable for an adequate development of coping with social challenges in the rat Developmental Psychobiology: The Journal of the International Society for Developmental Psychobiology 34:129-138

van Dixhoorn ID et al. (2016) Enriched housing reduces disease susceptibility to co-infection with porcine reproductive and respiratory virus 
(PRRSV) and Actinobacillus pleuropneumoniae (A. pleuropneumoniae) in young pigs PloS one 11:e0161832

White H, Richert B, Schinckel A, Burgess J, Donkin S, Latour M (2008) Effects of temperature stress on growth performance and bacon quality in grow-finish pigs housed at two densities Journal of Animal Science $86: 1789-1798$

Wood-Gush D, Beilharz R (1983) The enrichment of a bare environment for animals in confined conditions Applied Animal Ethology 10:209-217 


\section{Supplementary material}

Table 1: Means \pm SEM of the percentages of time spent on behaviours for which a sex $\times$ day interaction was found in female and male pigs over observation days.

\begin{tabular}{|c|c|c|c|c|c|c|}
\hline $\begin{array}{l}\text { Behaviour } \\
\text { (\% of time) }\end{array}$ & Sex & Day 49 & Day 54 & Day 60 & Day 78 & Day 125 \\
\hline \multirow[t]{3}{*}{ Inactivity } & $\mathrm{F}$ & $52.8 \pm 1.4^{\mathrm{a}}$ & $56.4 \pm 1.4^{\mathrm{ab}}$ & $58.2 \pm$ & $60.7 \pm 1.2^{\mathrm{b}}$ & $66.2 \pm 1.3^{\mathrm{c}}$ \\
\hline & & & & $1.2^{\mathrm{b}, \mathrm{x}}$ & & \\
\hline & M & $52.0 \pm 1.4^{\mathrm{a}}$ & $53.8 \pm 1.4^{\mathrm{a}}$ & $53.9 \pm 1.3^{\mathrm{a}, \mathrm{y}}$ & $59.0 \pm 1.6^{\mathrm{b}}$ & $69.3 \pm 1.2^{\mathrm{c}}$ \\
\hline \multirow[t]{2}{*}{ Chewing } & $\mathrm{F}$ & $11.0 \pm 0.8^{\mathrm{a}}$ & $10.9 \pm 0.8^{\mathrm{a}}$ & $8.2 \pm 0.5^{\mathrm{b}}$ & $8.2 \pm 0.5^{\mathrm{b}}$ & $7.6 \pm 0.6^{\mathrm{b}}$ \\
\hline & M & $10.4 \pm 0.7^{\mathrm{a}}$ & $10.3 \pm 0.8^{\mathrm{a}}$ & $8.1 \pm 0.5^{\mathrm{bc}}$ & $9.0 \pm 0.7^{\mathrm{ab}}$ & $6.1 \pm 0.5^{c}$ \\
\hline \multirow[t]{2}{*}{ Chewing substrates } & $\mathrm{F}$ & $8.1 \pm 0.9^{\mathrm{a}}$ & $8.0 \pm 0.9^{\mathrm{a}}$ & $5.4 \pm 0.6^{\mathrm{b}}$ & $5.2 \pm 0.6^{\mathrm{b}}$ & $5.1 \pm 0.6^{\mathrm{b}}$ \\
\hline & M & $7.4 \pm 0.8^{\mathrm{a}}$ & $7.5 \pm 0.9^{\mathrm{a}}$ & $5.4 \pm 0.6^{\mathrm{bc}}$ & $6.1 \pm 0.7^{\mathrm{ab}}$ & $4.1 \pm 0.5^{\mathrm{c}}$ \\
\hline \multirow[t]{2}{*}{ Manipulation } & $\mathrm{F}$ & $2.4 \pm 0.4^{\mathrm{ab}}$ & $2.4 \pm 0.4^{\mathrm{a}}$ & $1.7 \pm 0.3^{\mathrm{ab}}$ & $2.0 \pm 0.3^{\mathrm{ab}}$ & $1.2 \pm 0.2^{\mathrm{b}}$ \\
\hline & M & $1.9 \pm 0.3^{\mathrm{a}}$ & $2.7 \pm 0.3^{b}$ & $1.6 \pm 0.2^{\mathrm{a}}$ & $1.7 \pm 0.2^{\mathrm{a}}$ & $0.6 \pm 0.1^{\mathrm{c}}$ \\
\hline \multirow[t]{2}{*}{ Play } & $\mathrm{F}$ & $1.5 \pm 0.2^{\mathrm{a}}$ & $1.2 \pm 0.1^{\mathrm{ab}}$ & $1.1 \pm 0.1^{\mathrm{a}, \mathrm{x}}$ & $0.6 \pm 0.1^{\mathrm{bc}}$ & $0.5 \pm 0.1^{\mathrm{c}}$ \\
\hline & M & $1.6 \pm 0.2^{\mathrm{a}}$ & $1.4 \pm 0.2^{\mathrm{a}}$ & $1.9 \pm 0.1^{\mathrm{a}, \mathrm{y}}$ & $0.7 \pm 0.1^{\mathrm{b}}$ & $0.2 \pm 0.1^{\mathrm{b}}$ \\
\hline \multirow[t]{2}{*}{ Aggression } & $\mathrm{F}$ & $0.4 \pm 0.1^{\mathrm{x}}$ & $0.5 \pm 0.1^{\mathrm{x}}$ & $0.4 \pm 0.1^{\mathrm{x}}$ & $0.2 \pm 0.1^{\mathrm{x}}$ & $0.4 \pm 0.1$ \\
\hline & M & $1.4 \pm 0.1^{\mathrm{a}, \mathrm{y}}$ & $1.3 \pm 0.1^{\mathrm{a}, \mathrm{y}}$ & $1.2 \pm 0.1^{\mathrm{a}, \mathrm{y}}$ & $0.9 \pm 0.1^{\mathrm{a}, \mathrm{y}}$ & $0.4 \pm 0.1^{\mathrm{b}}$ \\
\hline \multirow[t]{2}{*}{ Mounting } & $\mathrm{F}$ & $0.2 \pm 0.1$ & $0.1 \pm 0.03$ & $0.1 \pm 0.04$ & $0.05 \pm 0.02$ & $0.06 \pm 0.02$ \\
\hline & M & $0.8 \pm 0.1$ & $0.7 \pm 0.1$ & $0.5 \pm 0.07$ & $0.4 \pm 0.08$ & $0.4 \pm 0.08$ \\
\hline
\end{tabular}

Only the behaviours affected by the sex $x$ day interaction $(\mathrm{p}<0.05)$ are given, other behaviours

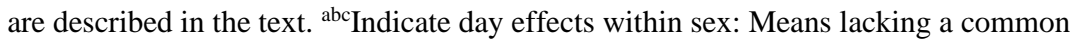
superscript letter $a, b$ or $c$ differ significantly $(\mathrm{p}<0.05)$ within the same sex. ${ }^{\mathrm{xy}}$ Indicate sex effects within day: Means having a different superscript letter $\mathrm{x}$, y differ significantly $(\mathrm{p}<0.05)$ within the same day. 



\title{
Chapter 5
}

\section{Effects of early and later life environmental enrichment and personality on attention bias in pigs (Sus scrofa domesticus)}

\author{
L. Luo ${ }^{1}$, I. Reimert ${ }^{1}$, E. N. de Haas ${ }^{1,2}$, B. Kemp ${ }^{1}$, J. E. Bolhuis ${ }^{1 *}$ \\ ${ }^{1}$ Adaptation Physiology Group, Department of Animal Sciences, \\ Wageningen University \& Research, the Netherlands \\ ${ }^{2}$ Current address: Department of Animals in Science and Society, Utrecht \\ University, $3584 \mathrm{CL}$, Utrecht, The Netherlands \\ *Corresponding author: J. E. Bolhuis \\ E-mail address: liesbeth.bolhuis@wur.nl
}

Published: Animal Cognition, 22.6: 959-972 


\subsection{Abstract}

We investigated effects of early and later life housing on attention bias, as an indicator of affective state, in pigs differing in coping style (reactive (LR) vs. proactive $(H R)$ ). Pigs $(n=128)$ in barren or enriched housing from birth (B1 vs. E1) that experienced either a switch in housing at 7 weeks of age or not (creating B1B2, B1E2, E1E2 and E1B2 treatments), were studied in a 180-sec attention bias test at 11 weeks. Pigs exposed to a 10-sec-auditory-andsudden-motion threat in the test arena paid more attention to the location of the threat, were more vigilant, showed less eating, more walking and were more likely to utter high-pitched vocalisations than non-threat pigs. During threat presence, HR pigs from post-switch enriched housing (E2-HR, i.e. $B 1 E 2+E 1 E 2)$ showed more vigilance but less exploration than others. After threat removal, no effects were found on time spent paying attention to the threat, vigilance, and eating, but E2-HR pigs paid attention to the threat more frequently, were more likely to utter high-pitched vocalisations and walked more compared to (part of) other groups, suggesting the most negative affective state in these animals. E2 pigs grunted more than B2 pigs. Thus, current housing, but not early life housing, affected behaviour in a personality-dependent manner in this attention bias test. Housing effects were opposite to expectation, possibly due to the short-term effect of the relative contrast between the home pens of the pigs and the test room. This potentially overruled putative long-term effects of environmental conditions on attention bias.

Key words: pigs, environmental enrichment, attention bias, affective states, coping style, early life 


\subsection{Introduction}

Affective states can influence cognitive processes, such as attention, memory, decision making and judgement. For example, a negative affective state can change expectations for the future and make animals more pessimistic (Paul et al. 2005), alter the motivation to consume a reward, enhance sensitivity to reward loss (Chaby et al. 2013), and increase attention towards negative stimuli (Lee et al. 2016). The form of cognitive bias most widely studied in non-human animals is judgement bias, in which the affective state of the animal influences its interpretation of ambiguous situations or cues (Mendl et al. 2009). In judgement bias tasks, animals need to be trained to discriminate between and respond to a positive and a negative stimulus before ambiguous stimuli are presented (Harding et al. 2004). This can be time-consuming and often not all animals can be successfully trained for the task (e.g. Verbeek et al. 2014). Besides, the training itself could be seen as cognitive enrichment and thereby, may influence affective state and overrule the mood-influencing factors under study (e.g. Roelofs et al. 2016). Attention bias tests, which do not require training, have been suggested to offer a faster and more practical method to assess negative affective states, like anxiety, in animals (Lee et al. 2016).

Negative affective states, such as anxiety, can result in an attention bias towards a potential threat (Lee et al. 2018). Indeed, in humans, affect-driven attention bias has been demonstrated, as individuals in high states of anxiety show greater attention towards threatening stimuli than non-anxious individuals (Bradley et al. 1997; Bradley et al. 1995). Also depression (Monk et al. 2018b) and chronic stress (Sipos et al. 2014) have been linked to altered 
threat perception. Attention bias tests have been conducted in a range of animals as well. For instance, sheep in an anxious state induced by an anxiogenic drug responded with increased vigilance and paid more attention to the previous location of a short-lived threat (a predator) than sheep in a reduced anxious state induced by an anxiolytic substance (Lee et al. 2016). They also showed a reduced willingness to consume the feed that was offered in the test, even after the threat had disappeared. Similarly, starlings that had been deprived of water bathing (and therefore likely in a negative affective state), showed more vigilance and less feeding following playback of an alarm call (Brilot and Bateson 2012).

Pigs kept in the barren housing conditions that are common in intensive pig farming show behavioural and physiological signs of chronic stress (Beattie et al. 2000; Bolhuis et al. 2006; de Jong et al. 2000), which could be accompanied by a negative affective state. Besides, it has been shown that negative experiences in early life can have long-term effects on behaviour, physiology, and cognition (Archard et al. 2012; Bolhuis et al. 2006; Chaby et al. 2013; Lukkes et al. 2009; Munsterhjelm et al. 2009b; Munsterhjelm et al. 2010; Sheriff et al. 2009). The effect of housing on affective state could thus not only depend on the current housing environment, but also on early life housing conditions. For instance, adult rats which were exposed to early life social isolation exhibited increased anxiety and conditioned fear behaviours, later in life (Lukkes et al. 2009). Also in pigs, long-term impacts of early life experiences have been found (see Telkänranta and Edwards 2017 for review), with sometimes favourable effects of enrichment at a young age on later life HPA-axis functioning and a decrease in aggressive behaviour in later life (Munsterhjelm et al. 2009a; Munsterhjelm et al. 2010). However, there are 
indications that pigs that switch from early life enriched housing to barren housing later on show as much or even more signs of poor welfare as pigs kept in a barren environment from birth onwards (Bolhuis et al. 2004; Munsterhjelm et al. 2009a), suggesting that a loss of enrichment may be more detrimental than barren housing throughout life. Thus, apart from a potential long-term negative effect of adverse early life conditions, also a change from a favourable to a suboptimal (barren) environment may result in a negative mood (Burman et al. 2008; Douglas et al. 2012).

Several studies suggest that personality (traits) may interact with affective state (Barnard et al. 2018; Lecorps et al. 2018) and, for instance, affect the attention bias for negative and positive stimuli (Segerstrom 2001). Part of the personality of humans and other animals is revealed in the way they cope with challenging situations, i.e. their coping style. Coping can be defined as the behavioural and physiological effort to master a situation (Koolhaas et al. 1999), where successful coping mainly depends on the controllability and predictability of the stressful condition (Koolhaas et al. 1999; Ursin and Olff 1995). Individuals show a wide variation in adaptive coping responses when exposed to the same stressful situation (Bolhuis et al. 2004; Koolhaas et al. 1999). Part of this variation is consistent across time and context, suggesting that the coping style of an individual is a stable personality trait (Koolhaas et al. 1999). Response patterns of individuals at either extreme end of their population are referred to as proactive vs. passive or reactive coping styles (Koolhaas 2008; Koolhaas et al. 1999). Proactive copers tend to use prior experience rather than present information, and are more likely to develop habits, ignore small environmental changes, and therefore seem less flexible to adapt to changing situations than reactive copers (Bolhuis et al. 2004; 
Koolhaas et al. 1999). On the other end of the scale, passive or reactive copers seem to keep a close eye to their environment, respond to even small changes and more flexibly adjust to changing conditions (Bolhuis et al. 2004; Koolhaas et al. 1999), and may therefore more easily adapt to the environment (Coppens et al. 2010). In pigs a divergence in coping responses can be assessed by exposing them to a back-test at an early age. The reaction of piglets to this manual restraint in supine position seems to reveal part of their coping style. The reaction to this test is heritable (Iversen et al. 2017; Velie et al. 2009; Zebunke et al. 2015). Pigs that struggle and squeal relatively much in this test, the "high-resisters" (HR) or proactive coping pigs, respond more actively to challenges (Reimert et al. 2013b; Reimert et al. 2014b; Ruis et al. 2001), are more rapid (Zebunke et al. 2015) but superficial in their exploration of novel stimuli (Jansen et al. 2009) and poorly adapt to a change in environment (Geverink et al. 2004). Pigs responding with relative immobility and silence in the back-test, the "low-resisters" (LR) or reactive coping pigs, on the other hand, are more cautious in exploration (Bolhuis et al. 2004; Jansen et al. 2009) and more flexible in adjusting their behaviour to changing conditions (Bolhuis et al. 2004; Geverink et al. 2004; Melotti et al. 2011). It has been suggested that pigs with a reactive coping style are more influenced by their housing environment, i.e. enriched versus barren (Asher et al. 2016; Bolhuis et al. 2003; Bolhuis et al. 2005a; Bolhuis et al. 2005b). For example, Asher et al. (2016) described that pigs with a more reactive personality responded more pessimistically in a judgement bias test when housed barren, but more optimistically when kept in a more enriched environment. Thus, when assessing the effects of early life and current 
housing conditions on the affective state of pigs, part of the individual variation found may be related to their personality type or coping style.

The aim of this study was to investigate the impacts of early life and current enrichment on the affective state of pigs using an attention bias test, and their potential interaction with the pigs' individual coping style.

Our attention bias test for pigs was based on a test recently developed for sheep (Lee et al. 2016; Monk et al. 2018b), involving a food reward as a positive stimulus and the attention paid to a threat as a measure of a negative affective state. This test in sheep has been shown to reflect anxiety as sheep treated with anxiolytic or anxiogenic drugs showed reduced and increased attention to the threat and vigilance, respectively (Lee et al. 2016). In our study, pigs housed in either barren or enriched housing in early life, either experiencing a switch in housing conditions at a later age or not, were exposed to a similar attention bias test. We hypothesized that barren housed pigs, and particularly those that had experienced enrichment in early life, would pay more attention to the threat, show more vigilance, and would be less willing to eat the food reward, because they were expected to have a more negative affective state (more anxious). Moreover, we predicted that the affective state of LR (reactive) pigs would be more affected by their housing conditions, with barren housed LR pigs and LR pigs experiencing a loss in enrichment in later life responding most negatively to the threat. 


\subsection{Materials and Methods}

The established principles of laboratory animal care and use were followed, as well as the Dutch law on animal experiments. The Animal Care and Use Committee of Wageningen University \& Research approved the experiment (DEC code: 2017.W-0001.001.IvD.3).

\subsubsection{Animal and housing}

Piglets (Tempo $\times$ Topigs 20) from 30 sows, divided over 2 batches equally and balanced for groups, were studied in this experiment. Sows were inseminated on the same day within a batch. From one month before farrowing, they were housed at the experimental facilities (Carus) of Wageningen University \& Research, Wageningen, the Netherlands. During the lactation period, 14 litters of piglets and their sows were housed in 8.6 $\mathrm{m}^{2}$ barren (B1) pens with a solid floor and a small area with slats. The other 16 litters of piglets were housed in enriched pens (E1) with the same $8.6 \mathrm{~m}^{2}$ part as in the barren pens, to which another $8.5 \mathrm{~m}^{2}$ area was added (total pen size $17.1 \mathrm{~m}^{2}$ ) that was enriched with $1.7 \mathrm{~kg}$ straw, $300 \mathrm{~L}$ of sawdust, and $270 \mathrm{~L}$ of peat. Extra, fresh straw and sawdust were added daily, and peat was added weekly ( $0.8 \mathrm{~kg} /$ day straw, $40 \mathrm{~L} /$ day sawdust, and $30 \mathrm{~L} /$ week peat) in the enriched part. Additionally, two toys hanging against the wall of the pen, one chain with a ball and one chain with screws that touched floor, were placed in the barren pens, and two toys, one chain with a ball and a toy that was alternated daily selected from 4 different toys, were placed in the enriched pens from 5 days after birth. All sows were housed in the same farrowing crates without access to the enrichment. In the first week after birth, one heating lamp was provided in the barren pens, and two lamps in 
the enriched pens. Each pen had one drinking nipple for the piglets and one for the sow. Sows were fed a standard commercial diet twice a day. From 5 days of age onwards, piglets received some fresh commercial feed. Temperature was set at $25^{\circ} \mathrm{C}$, and gradually decreased to $21^{\circ} \mathrm{C}$ over a course of 2 weeks. Each pen was cleaned daily and lights and a radio were on from 7:00 until 19:00 hours.

At 13 days of age, all piglets were subjected to a back-test to assess their coping style, also referred to as personality (Bolhuis et al. 2000). Briefly, in this test, piglets are restrained in supine position for $1 \mathrm{~min}$ and the number and latency of escape attempts and vocalisations are recorded (see Melotti et al. 2011 for details). In line with Reimert et al. (2014b), pigs were classified as relatively "high resisters" (HR) if they struggled 2 times and vocalized at least 25 times, or struggled at least 3 times, and as "low-resisters" (LR) if they struggled 0 or 1 time, or struggled 2 times and vocalized less than 25 times. In this selection criterion used, no extremes were selected, but rather the experimental pigs were either labelled as HR or LR (Melotti et al. 2011).

Pigs were weaned at 28 days of age, and 192 pigs (96 per batch) were selected and regrouped in 32 new pens containing 6 pigs each from different farrowing pens. The composition of each new group was balanced for sex, coping style and bodyweight. Housing treatment (B1 vs. E1) for each pig was kept the same as before weaning; thus, B1 pigs went to barren pens, which measured $5.6 \mathrm{~m}^{2}$ and had partly solid floor and partly slatted floor. The other half of pigs from E1 farrowing pens were housed in $11.2 \mathrm{~m}^{2}$ enriched pens with $2.5 \mathrm{~kg}$ straw, $400 \mathrm{~L}$ of sawdust, and $360 \mathrm{~L}$ of peat on the floor. Extra, fresh straw and sawdust were added daily, and peat was added weekly in 
enriched pens ( $1.25 \mathrm{~kg} /$ day straw, $60 \mathrm{~L} /$ day sawdust, and $45 \mathrm{~L} /$ week peat). From 39 days of age, enriched housed pigs also received extra enrichment (e.g. branches, jute sacks, egg trays, and ropes) on each Monday until the end of the experiment.

Each pen had one drinking nipple and pigs received solid commercial feed ad libitum. On the weaning day, the temperature was set at $25{ }^{\circ} \mathrm{C}$ and was decreased over the course of 2 weeks to $21^{\circ} \mathrm{C}$. It was kept at $21^{\circ} \mathrm{C}$ until the end of the experiment. After weaning, one heating lamp was provided in both barren and enriched pens for two weeks. Lights and a radio (channel with Dutch pop music and hourly news) were on from 7:00 until 19:00 hours.

At 47 days of age, pigs experienced either a switch in housing conditions (from barren to enriched or vice versa) or not, resulting in four treatment groups, E1E2, E1B2, B1E2, B1B2, $\mathrm{n}=8$ pens and 48 pigs per group. B1B2 and E1E2 groups were also moved to new pens to control for handling and relocation effects, but without a change in enrichment conditions. After this switch, straw, peat and toys were provided and added as described before, but only $30 \mathrm{~L}$ of sawdust was added daily.

\subsubsection{Attention bias test}

To assess attention bias in pigs, which could be an indicator of their affective state (negative valence related to anxiety), an attention bias test was carried out with 128 pigs, when they were around 76 days of age. Four pigs per pen were selected from E1E2, E1B2, B1E2, and B1B2 housing conditions, with equal numbers of each sex and coping style. 
Half of the pigs were exposed to a (non-social, unfamiliar) threat as experimental group, and the other half were not and served as control group, balanced for sex and housing conditions.

The test set-up was based on the sheep study from Lee et al. (Lee et al. 2016). Pigs were individually placed in a $5 \times 5 \mathrm{~m}$ test arena with solid walls away from their home pen (Figure 5.1). All pigs had been in the arena once before for another test at 6 weeks of age. In the centre of the arena, a metal bucket was placed containing feed (450 g of the pigs' normal feed) to which 8 chocolate peanuts and 10 pieces of carrots were added (which are generally preferred over normal feed). Testing order was balanced for housing, sex and coping style.

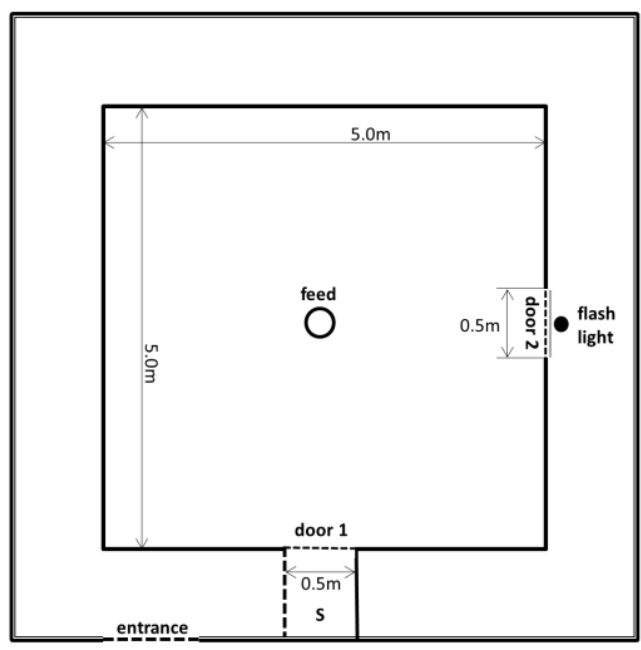

Figure 5.1: Layout of the room used for the attention bias test. S indicates a start box. Door 1 is the entrance and exit door to the test arena, and door 2 is a guillotine door in front of the plexiglass behind which the flashlight was placed. 
At the beginning of the test, a red and blue flashlight (off) was present outside the arena and was visible through a plexiglass window $\left(0.5 \mathrm{~m}^{2}\right)$, when guillotine door 2 was lifted, on the right side of the arena. Once a pig entered the test arena from door 1 , the door was shut behind it, and a timer was started. When the pig looked at the direction of the threat or maximum 10 sec after the pig entered the test arena, the flash light was turned on and door 2 was pulled up and down continuously (seven times), producing loud squeaking sounds, rapid movement while showing the flash light. This threat, i.e. the combination of the flashlight with the moving door and aversive sound, lasted for $10 \mathrm{sec}$. Hereafter, door 2 was softly closed and the flashlight was turned off. Pigs stayed in the test arena for $180 \mathrm{sec}$ in total and left the test arena from door 1 to go back to their home pen. The test was carried out over 2 days per batch.

Behaviour of the pigs, as well as attention towards the threat, were scored continuously using behaviour sampling with The Observer 5.0 software (Noldus Information Technology b.v., the Netherlands) (see Table 5.1 for the ethogram). In addition, the latency to eat after the threat had been removed was scored. One observer scored vocalisations, defecating and urinating of all pigs. Another single observer recorded all other behaviours of the pigs. Interrater reliability of behavioural observations was deemed substantial $($ Cohen's Kappa $=0.74)$. After removal of the threat, 17 pigs failed to eat, and their latency was set at the maximum time score. 
Table 5.1: Ethogram used to score the behaviours of the pigs during the attention bias test. Behaviours were scored as states unless indicated otherwise.

\begin{tabular}{|c|c|}
\hline Behaviour & Definition \\
\hline \multicolumn{2}{|l|}{ Attention class } \\
\hline $\begin{array}{l}\text { Attention to the } \\
\text { threat }\end{array}$ & With the head oriented toward the location of the threat \\
\hline $\begin{array}{l}\text { Attention to } \\
\text { something else }\end{array}$ & With the head oriented toward other directions, except threat \\
\hline \multicolumn{2}{|l|}{ Behaviour class } \\
\hline Vigilance & Standing motionless with head at shoulder height or higher or lower \\
\hline & $\begin{array}{l}\text { Eating food in the bucket. The eating event continues while the pig is chewing provided that the } \\
\text { head stays close to the bucket and the pig remains non-vigilant. Once the pig becomes vigilant }\end{array}$ \\
\hline Eating & $\begin{array}{l}\text { or moves away from the bucket, this is considered to be the end of eating, even if the pig } \\
\text { continues chewing }\end{array}$ \\
\hline Exploring & $\begin{array}{l}\text { Exploring the floor or wall of the arena by sniffing, nosing, licking or rooting it with the rooting } \\
\text { disc }\end{array}$ \\
\hline Walking & $\begin{array}{l}\text { Walking without performing any other described behaviour. All } 4 \text { legs move or the pig turns } \\
\text { around at the same spot without moving all } 4 \text { legs }\end{array}$ \\
\hline Standing & Standing with four paws on the floor without performing any other described behaviour \\
\hline $\begin{array}{l}\text { High-pitched } \\
\text { vocalizations }^{\mathrm{a}}\end{array}$ & Screams, squeals or grunt-squeals \\
\hline Short grunt ${ }^{a}$ & Grunt lasting less than $0.4 \mathrm{sec}$ (Fraser 1974; Kiley 1972) \\
\hline Long grunt ${ }^{\mathrm{a}}$ & Grunt lasting more than $0.4 \mathrm{sec}$ (Fraser 1974; Kiley 1972) \\
\hline Bark $^{a}$ & A low tone that sounds like "wuff" \\
\hline Defecating ${ }^{a}$ & Defecating \\
\hline Urinating ${ }^{\mathrm{a}}$ & Urinating \\
\hline Escapinga & The pig jumps to the wall or the door \\
\hline
\end{tabular}

${ }^{a}$ Were scored as events, all other behaviours as states. 


\subsubsection{Statistical analyses}

SAS (SAS 9.4, SAS Institute Inc.) was used for all statistical analyses. Preliminary analyses revealed no effects of testing day, therefore it was removed from the final models. Urinating was very rare ( $n=12$ pigs, after threat) and escape behaviour and barks were not observed at all and were therefore not analysed. Preliminary analysis also revealed that short grunts were rather rare (only $16 \%$ of all grunts in pigs exposed to the threat). Therefore, short and long grunts were summed as 'grunts'.

For assessing the effect of the threat, we first compared the behaviours of the threat vs. the non-threat (control) pigs over the whole $180 \mathrm{sec}$ test. To that end, a mixed linear model with presence of the threat and batch as fixed effects, and pen nested within pre-housing (i.e. housing before the switch at 47 days of age), post-housing (i.e. housing after the switch which was the current housing at the time of the attention bias test) and batch as random effect was used. High-pitched vocalisations were analysed as a 0-1 variable using a generalized mixed model with a binary distribution and logit link function.

Subsequently, we analysed the behaviours of the threat pigs both during the 10 -sec threat and for a $150-\mathrm{sec}$ period after the threat. For this, a mixed linear model was used with pre-housing, post-housing, coping style, their interactions, and batch as fixed effects, and pen nested within pre-housing, post-housing and batch as random effect. Rates of grunts per min and frequency of vigilance after the threat were square root transformed to obtain normality of residuals. In one of the treatment combination no highpitched vocalisations occurred at all. Therefore, high-pitched vocalisations 
were analysed as a 0-1 variable using Fisher's exact tests to compare treatment groups. Sex did not affect any of the variables and was therefore removed from the final models.

Significant interactions $(p<0.05)$ were further investigated with post hoc pairwise comparisons using the least square means, with Tukey correction for 3-way interactions. Results are presented as means \pm SEM.

\subsection{Results}

\subsubsection{Comparison of threat vs. non-threat (control) pigs}

Table 5.2 presents the behaviours of pigs exposed and pigs not exposed to the threat. Pigs that were exposed to the threat paid attention to the location of the threat for a longer time $\left(F_{1,95}=144.7, p<0.001\right)$. Besides, pigs that were exposed to the threat spent more time on vigilance behaviour $\left(F_{1,95}=19.8\right.$, $p<0.001)$, and walking $\left(F_{1,95}=6.5, p=0.013\right)$, less time on eating $\left(F_{1,95}=19.0\right.$, $p<0.001)$, and were more likely to utter high-pitched vocalisations $\left(F_{1,95}=7.9\right.$, $p=0.006$ ) than pigs without a threat during the $180 \mathrm{sec}$ test. No effects were found on time spent exploring, standing and on rates of grunting or defecating. 
Table 5.2: Means \pm SEM of the behaviours and vocalisations of pigs that were exposed to a threat and pigs that did not experience a threat during the 3-min test.

\begin{tabular}{llll}
\hline Behaviour & Threat & No threat & $p$-value \\
\hline Attention to the threat (\% of time) & $7.1 \pm 0.6$ & $0.5 \pm 0.1$ & $<0.001^{* * *}$ \\
Vigilance (\% of time) & $13.6 \pm 1.4$ & $6.7 \pm 1.0$ & $<0.001^{* * *}$ \\
Eating (\% of time) & $15.0 \pm 1.8$ & $27.8 \pm 2.4$ & $<0.001^{* * *}$ \\
Exploring (\% of time) & $39.4 \pm 2.6$ & $37.7 \pm 2.5$ & 0.624 \\
Walking (\% of time) & $19.6 \pm 1.6$ & $14.6 \pm 1.2$ & $0.013^{*}$ \\
Standing (\% of time) & $12.2 \pm 1.3$ & $13.0 \pm 1.3$ & 0.677 \\
High-pitched vocalisations (\% of pigs) & 29.7 & 9.4 & $0.006^{* *}$ \\
Total grunts (rpm) & $5.9 \pm 0.9$ & $4.5 \pm 0.6$ & 0.705 \\
Defecation (rpm) & $0.49 \pm 0.06$ & $0.42 \pm 0.05$ & 0.375 \\
\hline
\end{tabular}

${ }^{* * *} p<0.001,{ }^{* *} p<0.01,{ }^{*} p<0.05$

\subsubsection{Behaviour of the pigs exposed to the threat}

Behaviour of pigs exposed to the threat was mainly influenced by (trends for) the interaction between post-housing and coping style, and the interaction between pre-housing and post-housing, whereas only one three-way interaction was found (as described in the text below). Therefore, the posthousing $\times$ coping style interaction is highlighted in Figure 5.2 and 5.4; the prehousing $\times$ post-housing interaction is shown in Figure 5.3 and 5.5.

\subsubsection{Behaviour of the pigs during the $10-\mathrm{sec}$ threat}

Only 6 out of 64 pigs ate during the threat and only 9 out of 64 pigs showed the behaviour standing. Pigs did not vocalise or defecate during the threat.

Attention towards the threat tended to be affected by the pre-housing $x$ coping style interaction $\left(F_{1,27}=3.1, p=0.092\right)$. Time spent on vigilance behaviour was affected by coping style $\left(F_{1,27}=15.6, p<0.001\right)$ and the posthousing $\times$ coping style interaction $\left(F_{1,27}=5.8, p=0.023\right)$. Post hoc analysis 
showed that E2-HR pigs showed more vigilance behaviour than other pigs $(p<0.05$, Figure $5.2 \mathrm{~b})$. Exploration was affected by coping style $\left(F_{1,27}=5.2\right.$, $p=0.031)$ and the post-housing $\times$ coping style interaction $\left(F_{1,27}=6.7, p=0.014\right)$ and the pre-housing $\times$ post-housing $\times$ coping style interaction $\left(F_{1,27}=4.8\right.$, $p=0.036)$. E2-HR pigs spent less time on exploring than other pigs $(p<0.05$, Figure $2 \mathrm{c}$ ), but this effect was merely due to the large coping style contrast within E1E2 housing: HR pigs in E1E2 housing (1.9 $\pm 0.9 \%)$ explored much less than their E1E2-LR counterparts (47.2 $\pm 11.8 \%, p=0.012)$. The percentage of time spent on walking tended to be affected by the prehousing $\times$ post-housing interaction $\left(F_{1,27}=4.2, p=0.050\right)$.

\subsubsection{After the threat}

There were no housing or coping style effects on time spent on attention to the threat location, vigilance, and eating after the threat was removed. The frequency of paying attention to the threat, however, was affected by the post-housing $\times$ coping style interaction $\left(F_{1,28}=5.1, p=0.031\right)$, with higher levels for E2-HR (2.6 \pm 0.4$)$ than for E2-LR pigs $(1.4 \pm 0.3, p=0.045)$ and levels of B2 pigs in between (B2-HR: $1.7 \pm 0.4$; B2-LR: $2.3 \pm 0.5$ ). Changing from vigilance to other behaviours tended to be affected by post-housing $\times$ coping style $\left(F_{1,28}=3.2, p=0.086\right)$. E2 pigs tended to start eating sooner $(63.4 \pm 9.2 \mathrm{sec})$ than B2 pigs after the threat had ended $\left(95.3 \pm 10.1 \mathrm{sec}, F_{1,27}=4.1, p=0.052\right)$ and exploring tended to be affected by the pre-housing $\times$ post-housing interaction $\left(F_{1,27}=3.6, p=0.068\right)$. Walking tended to be affected by posthousing $\left(F_{1,27}=4.0, p=0.057\right)$, and was affected by the post-housing $\times$ coping style interaction $\left(F_{1,27}=6.3, p=0.018\right)$. Post hoc analysis showed that $\mathrm{E} 2-\mathrm{HR}$ pigs walked more than E2-LR pigs $(p=0.029)$ and B2-HR pigs $(p=0.006$, Figure 
$5.4 \mathrm{~g})$. The percentage of time spent on standing was affected by pre-housing $\times$ post-housing $\left(F_{1,27}=4.8, p=0.038\right)$. E1B2 pigs $(19.4 \pm 4.1 \%)$ stood more than E1E2 pigs $(9.7 \pm 1.6 \%, p=0.047)$, with levels of B1B2 pigs $(10.4 \pm 2.6 \%)$ and B1E2 pigs $(15.6 \pm 2.7 \%)$ in between. E2 pigs grunted more often $(9.8 \pm 2.0$ per min) than B2 pigs ( $3.5 \pm 0.9$ per $\min )\left(F_{1,27}=9.1, p=0.006\right)$. The proportion of pigs that displayed high-pitched vocalisations was also higher in E2 housing than in $B 2$ housing $\left(F_{1,27}=6.5, p=0.029\right)$, but only so in the HR pigs ( $p=0.013$ for post-housing effect within HR pigs, see Figure $5.4 f)$. The rate of defecating per min tended to be affected by the pre-housing $\times$ post-housing interaction $\left(F_{1,27}=3.7, p=0.065\right)$. 

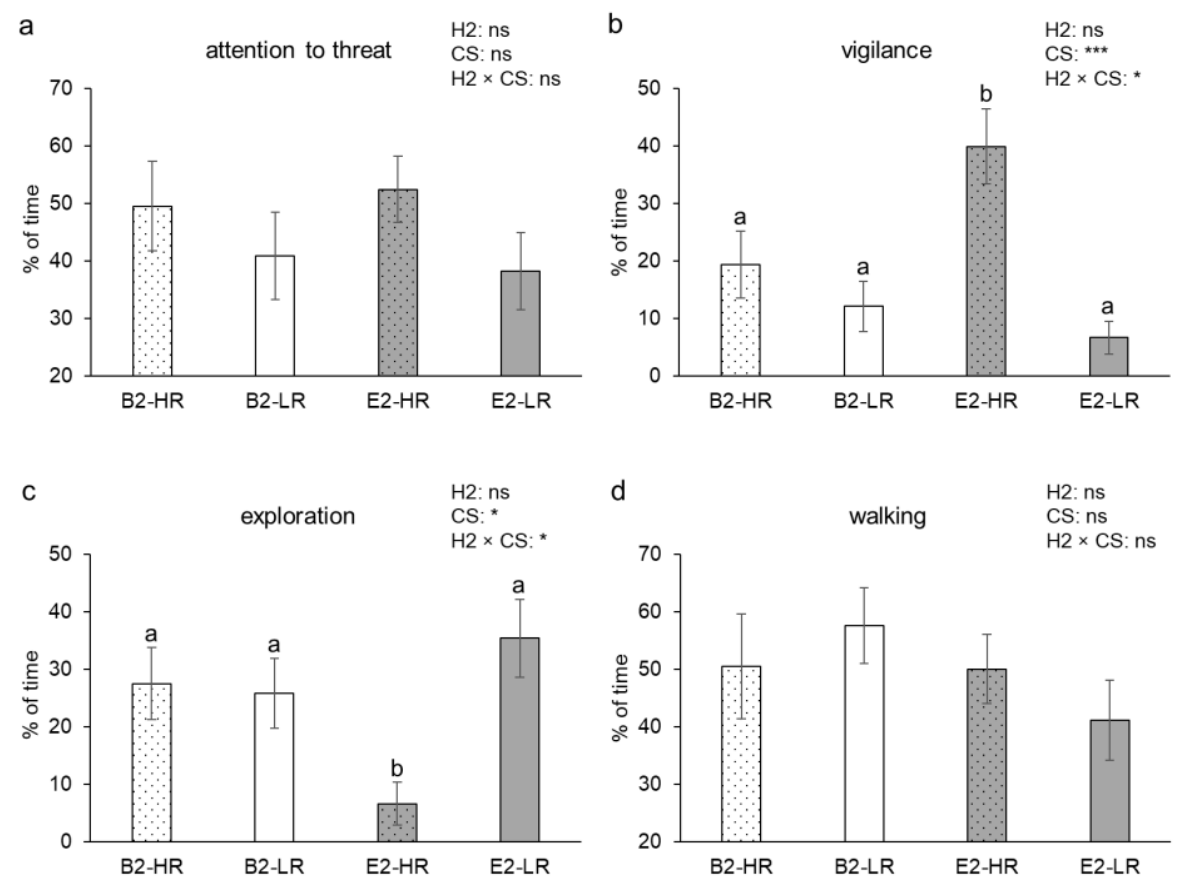

Figure 5.2: Percentages (means \pm SEM) of time spent on behaviours during the presence of the threat for high-resister (HR) vs. low-resister (LR) pigs housed in barren (B2) and enriched (E2) conditions. B2 and E2 refer to all pigs in barren housing or enriched housing from 7 weeks of age, respectively, irrespective of their previous housing. Panels $a, b, c$ and $d$ show the percentage of time spent on attention towards the threat, vigilance, exploration and walking, respectively. Significances of posthousing $(\mathrm{H} 2)$, coping style $(\mathrm{CS})$, and the post-housing $\times$ coping style $(\mathrm{H} 2 \times \mathrm{CS})$ interaction are indicated by ${ }^{* * *} p<0.001,{ }^{*} p<0.05$, and non-significance is ns. Groups lacking a common letter $(a, b)$ significantly differ $(p<0.05)$. 

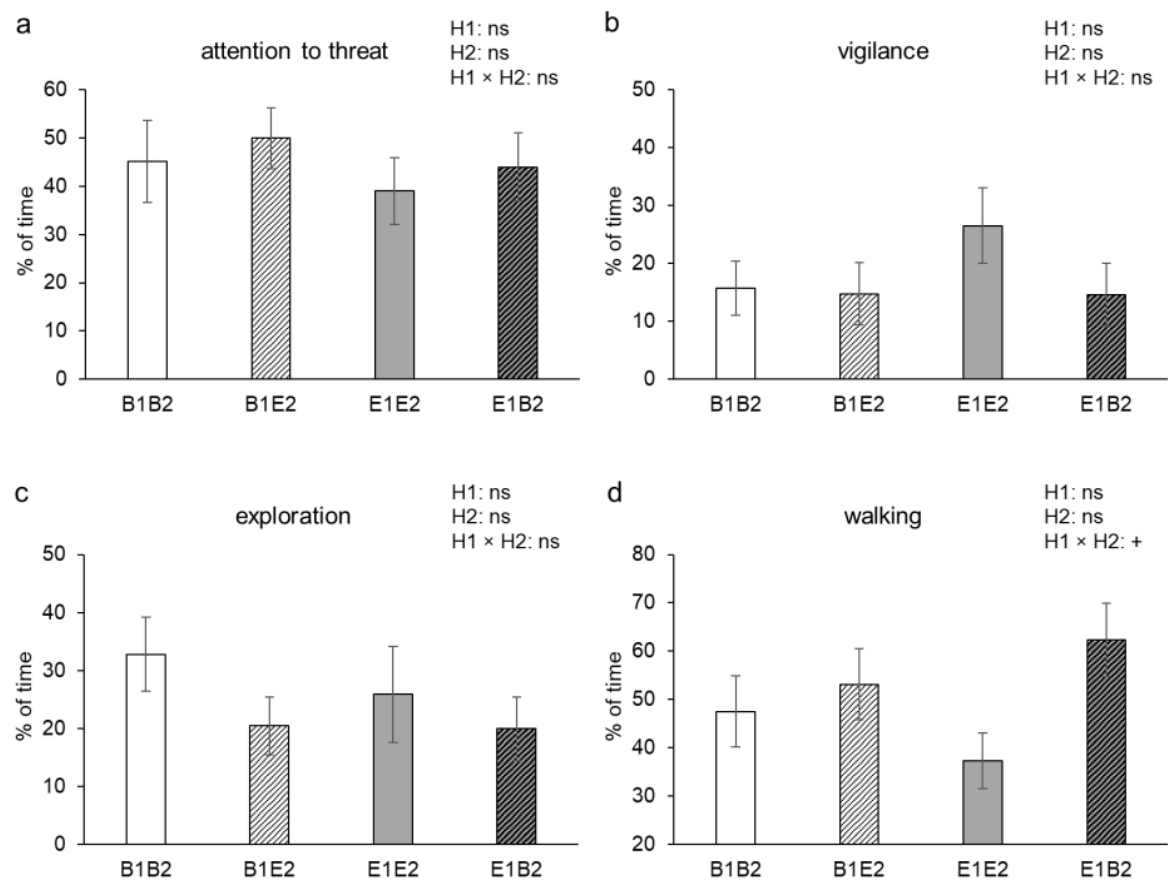

Figure 5.3: Percentages (means \pm SEM) of time spent on behaviours during the presence of the threat for pigs housed in four different housing conditions (B1B2 and E1E2: housed in barren and enriched pens, respectively, throughout life; B1E2 and E1B2: experienced a switch in housing conditions from barren to enriched or vice versa at 7 weeks of age). Panels $a, b, c$ and $d$ show the percentage of time spent on attention towards the threat, vigilance, exploration and walking for pigs during the presence of the threat. Tendency of the pre-housing $\times$ post-housing $(\mathrm{H} 1 \times \mathrm{H} 2)$ interaction is indicated by $+p<0.10$, and non-significance is ns. 

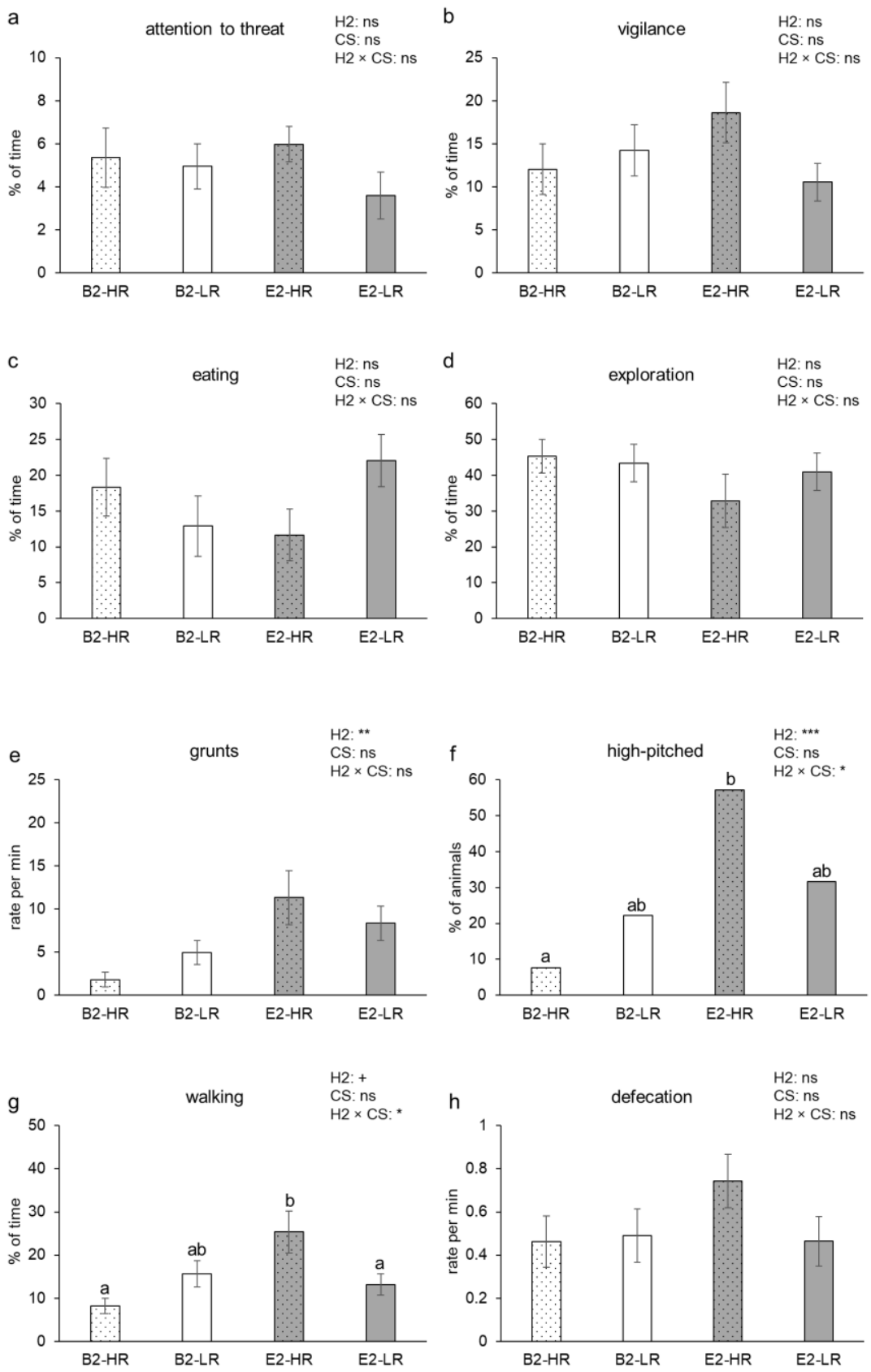
Figure 5.4: Means \pm SEM of behaviours after removal of the threat $(150 \mathrm{sec})$ for highresister (HR) vs. low-resister (LR) pigs housed in barren (B2) and enriched (E2) conditions. B2 and E2 refer to all pigs in barren housing or enriched housing from 7 weeks of age, respectively, irrespective of their previous housing. Panels $a, b, c, d$, and $g$ show the percentage of time spent on attention towards the threat, vigilance, eating, exploration, and walking, respectively; panels e and $\mathrm{h}$ show the rate of grunts and defecation per min, and panel $f$ shows the percentage of pigs making highpitched vocalisations. Significances of post-housing $(\mathrm{H} 2)$, coping style (CS), and the post-housing $\times$ coping style $(\mathrm{H} 2 \times \mathrm{CS})$ interaction are indicated by $* * * p<0.001$, ${ }^{* *} p<0.01$, and $* p<0.05$; tendency is indicated by $+p<0.10$, and non-significance is ns. Groups lacking a common letter $(a, b)$ significantly differ $(p<0.05)$. 

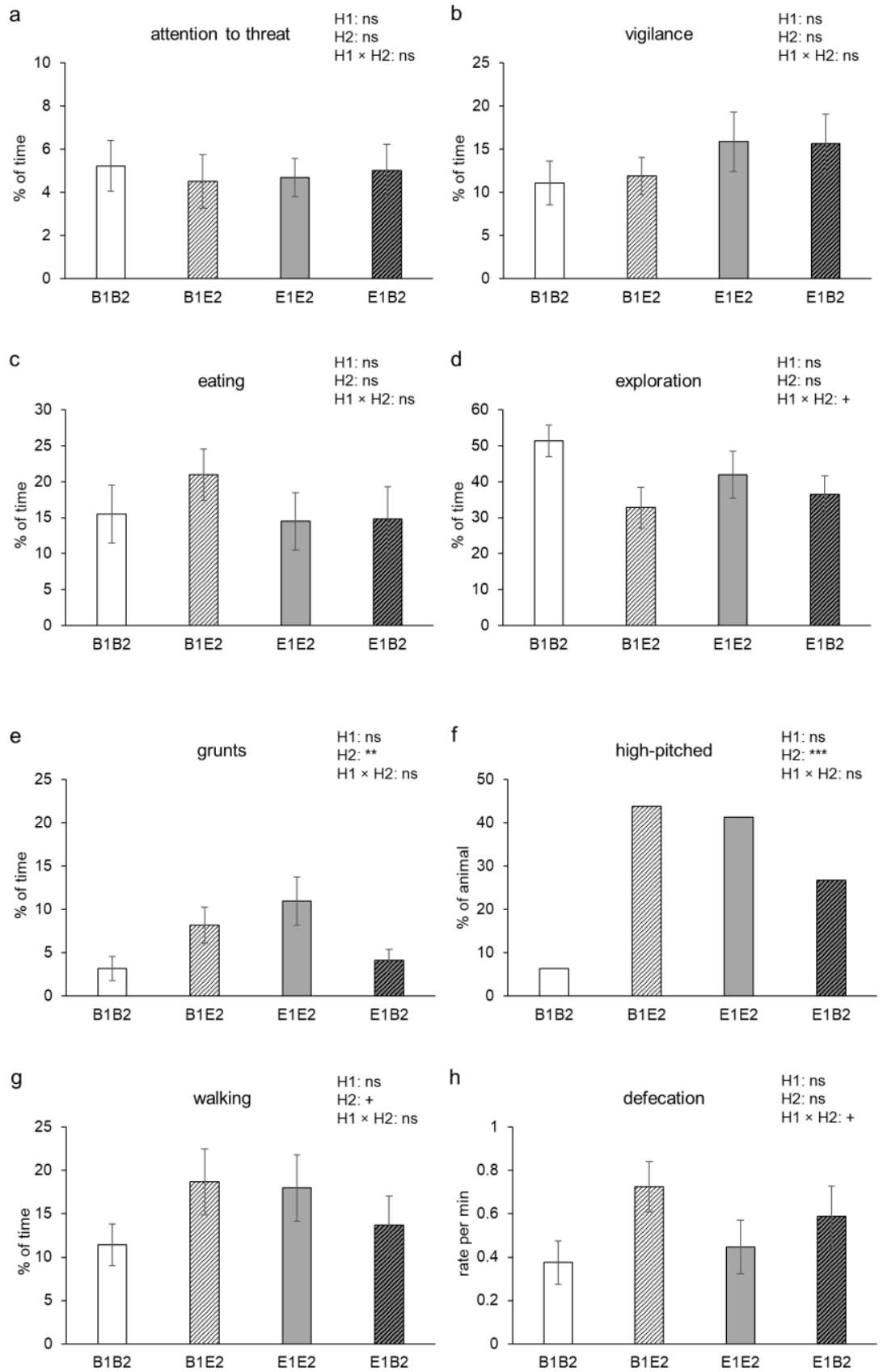
Figure 5.5: Means \pm SEM of behaviours for pigs housed in four different housing conditions (B1B2 and E1E2: housed in barren and enriched pens, respectively, throughout life; B1E2 and E1B2: experienced a switch in housing conditions from barren to enriched or vice versa from 7 weeks of age). Panels $a, b, c, d$, and g show the percentage of time spent on attention towards the threat, vigilance, eating, exploration, and walking, respectively; panels e and h show the rate of grunts and defecation per min; and panel $f$ shows the percentage of pigs making high-pitched vocalisations. Significances of pre-housing $(\mathrm{H} 1)$, post-housing $(\mathrm{H} 2)$, and the prehousing $\times$ post-housing $(\mathrm{H} 1 \times \mathrm{H} 2)$ interaction are indicated by $* * * p<0.001,{ }^{* *} p<0.01$; tendency is indicated by $+p<0.10$, and non-significance is ns.

\subsection{Discussion}

In this study, we investigated the impacts of early life and current enrichment on attention bias in pigs with diverging coping styles. We hypothesised that pigs from barren housing conditions, especially those that had experienced enrichment in early life, would pay more attention to the non-social, unfamiliar threatening stimulus, show more vigilance, and would be less willing to eat, because they were expected to have a more negative, anxious affective state. Besides, LR (reactive) pigs were expected to be more affected by their housing environment than HR (proactive) pigs.

\subsubsection{Response to the threat}

Pigs exposed to the 10 -sec threat approximately doubled the time spent on vigilance behaviour and halved time spent eating. Moreover, they paid more attention towards the location where the threat had been in the test room than pigs that were not exposed to the threat, and were more likely to display 
high-pitched vocalisations, which indicates that the pigs clearly responded to the threat, also after it had disappeared. It has been shown in previous studies that animals with a more negative affective state were more vigilant, less willing to eat, and paid more attention to the location of a threat (Brilot and Bateson 2012; Lee et al. 2018; Lee et al. 2016), which is parallel with what we found in this study, and confirms that the threat, a combination of a flash light and a moving guillotine door producing squeaking sounds, was aversive for pigs. Similar to human studies (Bar-Haim et al. 2007) and sheep studies (Lee et al. 2016; Monk et al. 2018b), attention towards a threat and vigilance were our key measures of attentional-orienting.

\subsubsection{Effects of early life conditions}

Based on previous behavioural studies (Bolhuis et al. 2005a; Douglas et al. 2012), we expected barren housed pigs (B2) to be in a more negative affective state than enriched housed pigs and thus to pay more attention to the threat (measured as head-orientation) and be more vigilant (see below for discussion on effects of current housing). We furthermore hypothesized this to be even more so for pigs originating from an enriched early life environment (E1B2) that thus had experienced a downgrade change in housing conditions (Bolhuis et al. 2005a; Douglas et al. 2012; Munsterhjelm et al. 2009a). The absence of interactions between early and later life housing, and the absence of an effect on attention to the threat or general vigilance, however, do not point to such an effect of a negative switch. Besides, we found no indications of a beneficial effect of early life enrichment either, which could mean that housing conditions in early life do not have long-term effects on affective state, or at least not on anxiety. Alternatively, potential 
effects of early life housing (up till 7 weeks of age) on the behavioural responses in the test may have been overruled by the later housing conditions the pigs had been kept in for 4 weeks at the time of attention bias testing (11 weeks of age). In another study we did find, though, that pigs from enriched housing in early life responded less strongly to reward loss in a successive negative contrast test carried out 8 weeks after a change in housing conditions (Luo et al., submitted), which could point to a more positive affective state in these animals.

\subsubsection{Effects of current housing}

The effect of later life environmental conditions, i.e. housing at the time of testing, on behaviour in the attention bias test were also not in line with our expectations and other studies, and, moreover, partly depended on coping style. During the threat, enriched housed HR pigs were more vigilant than other pigs. They also spent less time on exploration, particularly the HR pigs kept in enriched pens from birth onwards. After the threat, no effects were found on time spent on attention to the (location of the) threat or vigilance, but HR pigs from enriched pens more frequently paid attention to the threat than LR pigs from enriched pens. Enriched pigs grunted more often and, within the HR pigs, they also were more likely to display high-pitched vocalisations like squeals. Increased grunting and squealing have been described to occur during aversive events in which pigs were deprived of (visual and tactile) social contact (Reimert et al. 2013a). Especially highfrequency calls seem to be indicative of fear and other negative emotions in pigs when socially isolated (Leliveld et al. 2017). Long grunts (also called low grunts (Leliveld et al. 2017) were the most uttered vocalisations during the 
test and have been suggested to be used in social isolation as an attempt to make contact with group members (Kiley 1972; Leliveld et al. 2017). Possibly, the higher grunting rate in E2 pigs is related to a higher motivation to get into contact with their pen mates. Taken together, with the exception of a trend for a longer latency to start eating after the threat in barren housed pigs as compared with pigs from enriched housing, our results do not point to a more negative long-term affective (anxious) state in enriched animals. This could mean that barren housing does not induce a long-term negative mood. This seems unlikely, though, given the results of previous studies. Barren housed pigs have been reported to experience more chronic stress (Bolhuis et al. 2005a; Bolhuis et al. 2006), and to have a more negative affective state (Douglas et al. 2012) than enriched housed pigs, although it should be noted that another study reported no effects of barren housing on judgement bias in spite of clear negative effects on physiological and behavioural welfare indicators (Carreras et al. 2016). In a study in sheep, chronic stress, which was confirmed by HPA-axis dysregulation, against expectations reduced rather than increased vigilance to a predator threat (Verbeek et al. 2019). Explanations given for the lack of effects or effects opposite to expectations in this sheep study and in a quail study were a poor sensitivity of the test, or the context of the test (e.g. test arena and handling) which may have overruled the putative long-term effects of housing conditions on mood (Horváth et al. 2016; Verbeek et al. 2019). The latter could hold for our study as well, as pigs from barren housing may have experienced the release from their suboptimal environment to the test arena as more positive than enriched housed pigs. Several studies suggest that negative events, such as sheering (Sanger et al. 2011) and restraint plus social isolation in sheep 
(Doyle et al. 2010), before testing, result in more positive affective states during testing (i.e., a positive contrast between the test situation and the previous situation). It is possible that a long-term negative situation (like barren housing) preceding testing has a similar effect. In support of this, a study on rats reported a relatively positive affective state of barren housed individuals during a mood test (Mitchell et al. 2012).

When investigating the impact of housing conditions in group-housed animals on attention bias, the behavioural response in the test might thus potentially be influenced by the relative contrast between the test room/situation and the home environment, as well as by social isolation. Indeed, in the most convincing studies on the effect of mood on attention bias towards threatening stimuli, negative affective states were induced by pharmacological interventions (e.g. by administration of diazepam and $\mathrm{m}$ CPP) in sheep (Lee et al. 2016; Monk et al. 2018b) and rats (Wright and Rodgers 2014)). In these studies, the relative contrast between the home and test environment was the same for all animals, and the drugs remained active for the duration of the test, which likely circumvented a potential short-term influence of the test context on emotional state. Furthermore, Verbeek et al. (2019) suggested that chronic stress may alter the motivation to obtain feed rewards which could also interfere with the test outcome. In a successive negative contrast test we did find indications for a lower reward sensitivity in pigs housed in barren conditions from birth onwards as compared with pigs that had switched from enriched to barren pens and with enriched housed pigs (Luo et al., submitted). Finally, it has been suggested that individuals suffering from chronic stress or depression are less responsive to stimuli - either negative or positive - in general (Fureix et al. 
2012) which could also explain the lack of a clear housing effect on attention bias.

\subsubsection{Effects of coping style and its interaction with housing}

In humans, cognitive biases are dependent on both current mood and personality (Marshall et al. 1992). Classically, humans scoring high on the personality dimension neuroticism more often and strongly experience negative affect, whereas extraversion is associated with frequent and intense expression of positive emotions (Winter and Kuiper 1997). These personality traits thus possibly impact long-term mood. We studied a personality trait in pigs, i.e. their coping style, which is not inherently expected to be linked with a bias towards either negative or positive affect. Indeed, it has been suggested that the dimension of coping style is independent of an emotionality dimension (Koolhaas et al. 2007) and rather reflects how individuals respond when challenged. Proactive, i.e. HR, pigs consistently have been shown to vocalise more often in challenging situations (Geverink et al. 2002; Jansen et al. 2009; Reimert et al. 2014a; Reimert et al. 2014b; Ruis et al. 2001) than reactive, i.e. LR, pigs, and to display more locomotion (Jansen et al. 2009; Reimert et al. 2013b; Reimert et al. 2014b). LR pigs, on the other hand, have been reported to show more vigilance (Reimert et al. 2014a) and continue to do so even in an increasingly familiar environment (Jansen et al. 2009). Both of these response patterns can lead to successful coping with a challenging situation, with the style of reactive copers being more suited for changing environments.

In our study, the impact of housing on the behaviour of the pigs during the test depended on their coping style (and vice versa), in spite of the fact that 
we did not select the extremes of the tested population but labelled all pigs. We found the highest levels of walking and squealing in HR pigs from enriched housing after removal of the threat. This could, on the one hand, reflect the typical mode of responding of HR pigs, or, alternatively, indicate a more negative affective (anxious) state as compared with barren housed pigs and enriched housed LR pigs. We tentatively suggest the latter, as enriched HR pigs also showed significantly more vigilance during the threat and less exploration. In addition, after the threat they more often paid attention to the threat than LR pigs from enriched housing, and numerically spent the most time on vigilance and attention towards the location of the threat and had the highest frequency of defecating, which all have been linked with aversive situations and/or seem to reflect negative emotions (Bouissou and Vandenheede 1995; Brilot and Bateson 2012; Lee et al. 2016; Reimert et al. 2013a). The higher impact of housing on the behaviour of HR pigs in this test is not in line with studies suggesting that proactive copers are less responsive to the distinction between barren and enriched housing than reactive copers. For instance, LR pigs were reported to show more play behaviour in enriched pens, but more oral manipulation of pen mates (e.g. tail and ear biting) and gastric lesions in barren pens than HR pigs (Bolhuis et al. 2005a; Bolhuis et al. 2006). Moreover, Asher et al. (2016) recently found reactive copers to respond more optimistically in a judgement bias test when housed enriched and more pessimistically when kept in barren pens, suggesting that, apart from behaviour, also the affective state of reactive copers is more affected by (lack of) enrichment than that of proactive copers.

It should be noted, though, that HR pigs, albeit seemingly less affected by barren housing, show more difficulty in coping with relocation and social 
isolation (Bolhuis et al. 2004; Geverink et al. 2004; Ruis et al. 2001). If the behaviour in our test reflected the acute response to the test setting (involving relocation and social isolation) rather than the long-term impact of housing conditions on affective state, this might explain why (enriched) HR pigs were most affected. As argued before, in barren pigs this effect may have been attenuated by the exposure to a spacious, stimulus-rich test room contrasting their small, barren home pens. Therefore, for future studies on mood in animals, it is worthwhile to consider that (1) personality traits (e.g. anxiety) may influence a long-term affective state per se; (2) personality traits (e.g. coping style) may, irrespective of affective state, influence the mode of responding of animals which could interfere with the read-out variables of a test for affective state; (3) personality traits may influence both the impact of the long-term condition (e.g. housing, chronic stress) under study, as well as that of the test setting.

\subsubsection{Remarks on the test design and measurements}

Our test set-up was based on a paradigm developed for sheep that has been successfully validated by pharmacological interventions (Lee et al. 2016; Monk et al. 2018a). In the sheep studies, a dog was effectively used as a threatening stimulus (albeit habituation to dogs in daily life may reduce its threatening potential for sheep, as argued by Verbeek et al. (2019)). To our knowledge, this is the first study on attention bias in pigs, for which it is harder to find a threatening stimulus. We used a squeaking moving guillotine door and a flashlight. We found clear evidence, by comparison with pigs not exposed to this threat, that the pigs were negatively affected by this stimulus, even after its disappearance, but we cannot rule out that stronger and/or 
longer threats would have given more clear results. In addition, attention towards the threat was in our study based on the position of the head (oriented toward the location of the threat) and not on glancing. In human studies, however, 'attention' refers to overt visual attention, typically based on eye gaze (Crump et al. 2018), which we found more difficult to score in an objective manner but may nevertheless have been a more accurate indicator of attention bias. This could be a common concern for previously published studies in freely moving farm animals, in which attention was also measured as the 'head oriented towards the threat' (e.g Lee et al. 2016; Verbeek et al. 2019). Thus, rather than scoring attention bias in the narrow sense (referring to vision), our test may thus have picked up general emotional reactivity to the threat, which might alternatively be referred to as 'altered perception of the threat'. It should be noted, though, that our threat stimulus comprised both visual and auditory cues, and that for pigs (and several other farm animals) other sensory modalities may be as much or even more relevant than vision alone, perhaps asking for a more broad definition of attention bias and corresponding measurements. Finally, food was used as a positive stimulus, but motivation for food can vary considerably between animals and within animals over the span of a single day (Monk et al. 2018a), and potentially be affected by housing or stress (see above) so, even though we balanced the test order for our treatments, food may not be an optimal positive stimulus in an attention bias test for pigs. Further studies are required to develop a practical attention bias test in pigs, and to circumvent (or reliably pinpoint) potential confounding effects of the test set-up and test read-outs with personality traits and the long-term treatments, like housing conditions, under study. 


\subsection{Conclusion}

Pigs responded to a short-lived threat during an attention bias test by increasing vigilance, head-orientation towards the (previous) location of the threat, high-pitched vocalisations and reduced time spent eating, revealing increased anxiety. Current, but not early life, housing affected the behaviour in this test in a personality-dependent manner. Although no effects on duration of attention to the threat or vigilance were found, other behavioural variables (e.g. the frequency of attention toward the threat and high-pitched vocalisations) suggest a more negative emotional state following exposure to the threat in HR pigs from enriched housing. The housing effect was opposite to what we expected, which might be explained by a short-term effect of the relative contrast between the home pens of the pigs and the test room. This potentially overruled putative long-term effects of environmental conditions on mood.

\subsection{Acknowledgements}

The authors are grateful to Caroline Lee for valuable advice on the test setup. We also would like to thank Manon van Marwijk, Monique Ooms, the animal caretakers and students for skilful assistance in conducting the experiment. The authors also appreciate the sponsor for Lu Luo from the China Scholarship Council.

\subsection{Compliance with ethical standards}


5.9 Conflict of interest The authors declare that they have no conflict of interest.

5.10 Ethical approval All applicable institutional guidelines for the care and use of animals were followed. All procedures performed in this study involving animals were in accordance with the ethical standards of the institution or practice at which the study was conducted. 


\subsection{References}

Archard GA, Earley RL, Hanninen AF, Braithwaite VA (2012) Correlated behaviour and stress physiology in fish exposed to different levels of predation pressure Functional Ecology 26:637-645

Asher L, Friel M, Griffin K, Collins LM (2016) Mood and personality interact to determine cognitive biases in pigs Biology letters 12:20160402

Bar-Haim Y, Lamy D, Pergamin L, Bakermans-Kranenburg MJ, Van ljzendoorn $\mathrm{MH}$ (2007) Threat-related attentional bias in anxious and nonanxious individuals: a meta-analytic study Psychological bulletin 133:1

Barnard S, Wells DL, Milligan AD, Arnott G, Hepper PG (2018) Personality traits affecting judgement bias task performance in dogs (Canis familiaris) Scientific reports 8

Beattie V, O'Connell N, Kilpatrick D, Moss B (2000) Influence of environmental enrichment on welfare-related behavioural and physiological parameters in growing pigs Animal Science 70:443-450

Bolhuis J, Schouten W, De Jong I, Schrama J, Cools A, Wiegant V (2000) Responses to apomorphine of pigs with different coping characteristics Psychopharmacology 152:24-30

Bolhuis JE, Parmentier HK, Schouten WG, Schrama JW, Wiegant VM (2003) Effects of housing and individual coping characteristics on immune responses of pigs Physiology \& behavior 79:289-296

Bolhuis JE, Schouten WG, de Leeuw JA, Schrama JW, Wiegant VM (2004) Individual coping characteristics, rearing conditions and behavioural flexibility in pigs Behavioural brain research 152:351-360

Bolhuis JE, Schouten WG, Schrama JW, Wiegant VM (2005a) Behavioural development of pigs with different coping characteristics in barren and substrate-enriched housing conditions Appl Anim Behav Sci 93:213-228

Bolhuis JE, Schouten WG, Schrama JW, Wiegant VM (2005b) Individual coping characteristics, aggressiveness and fighting strategies in pigs Anim Behav 69:1085-1091

Bolhuis JE, Schouten WG, Schrama JW, Wiegant VM (2006) Effects of rearing and housing environment on behaviour and performance of pigs with different coping characteristics Appl Anim Behav Sci 101:68-85

Bouissou M-F, Vandenheede M (1995) Fear reactions of domestic sheep confronted with either a human or a human-like model Behavioural processes 34:81-92 
Bradley BP, Mogg K, Lee SC (1997) Attentional biases for negative information in induced and naturally occurring dysphoria Behaviour research and therapy 35:911-927

Bradley BP, Mogg K, Millar N, White J (1995) Selective processing of negative information: Effects of clinical anxiety, concurrent depression, and awareness Journal of abnormal psychology 104:532

Brilot BO, Bateson M (2012) Water bathing alters threat perception in starlings Biology letters:rsbl20111200

Burman OH, Parker RM, Paul ES, Mendl M (2008) Sensitivity to reward loss as an indicator of animal emotion and welfare Biology Letters 4:330333

Carreras R et al. (2016) Housing conditions do not alter cognitive bias but affect serum cortisol, qualitative behaviour assessment and wounds on the carcass in pigs Appl Anim Behav Sci 185:39-44

Chaby L, Cavigelli S, White A, Wang K, Braithwaite V (2013) Long-term changes in cognitive bias and coping response as a result of chronic unpredictable stress during adolescence Frontiers in human neuroscience 7:328

Coppens CM, de Boer SF, Koolhaas JM (2010) Coping styles and behavioural flexibility: towards underlying mechanisms Philosophical Transactions of the Royal Society B: Biological Sciences 365:40214028

Crump A, Arnott G, Bethell E (2018) Affect-Driven Attention Biases as Animal Welfare Indicators: Review and Methods Animals 8:136

de Jong IC, Prelle IT, van de Burgwal JA, Lambooij E, Korte SM, Blokhuis HJ, Koolhaas JM (2000) Effects of environmental enrichment on behavioral responses to novelty, learning, and memory, and the circadian rhythm in cortisol in growing pigs Physiology \& behavior 68:571-578

Douglas C, Bateson M, Walsh C, Bédué A, Edwards SA (2012) Environmental enrichment induces optimistic cognitive biases in pigs Appl Anim Behav Sci 139:65-73

Doyle RE, Fisher AD, Hinch GN, Boissy A, Lee C (2010) Release from restraint generates a positive judgement bias in sheep Appl Anim Behav Sci 122:28-34

Fraser D (1974) The behaviour of growing pigs during experimental social encounters The Journal of Agricultural Science 82:147-163 
Fureix C, Jego P, Henry S, Lansade L, Hausberger M (2012) Towards an ethological animal model of depression? A study on horses PLoS One 7:e39280

Geverink N, Heetkamp M, Schouten W, Wiegant V, Schrama J (2004) Backtest type and housing condition of pigs influence energy metabolism Journal of Animal Science 82:1227-1233

Geverink N, Schouten W, Gort G, Wiegant V (2002) Individual differences in aggression and physiology in peri-pubertal breeding gilts Appl Anim Behav Sci 77:43-52

Harding EJ, Paul ES, Mendl M (2004) Animal behaviour: cognitive bias and affective state Nature 427:312

Horváth M, Pichová K, Koštál L' (2016) The effects of housing conditions on judgement bias in Japanese quail Appl Anim Behav Sci 185:121-130

Iversen M, Bolhuis J, Camerlink I, Ursinus W, Reimert I, Duijvesteijn N (2017) Heritability of the backtest response in piglets and its genetic correlations with production traits animal 11:556-563

Jansen J, Bolhuis JE, Schouten WG, Spruijt BM, Wiegant VM (2009) Spatial learning in pigs: effects of environmental enrichment and individual characteristics on behaviour and performance Anim Cogn 12:303315

Kiley M (1972) The vocalizations of ungulates, their causation and function Zeitschrift für Tierpsychologie 31:171-222

Koolhaas J (2008) Coping style and immunity in animals: making sense of individual variation Brain, behavior, and immunity 22:662-667

Koolhaas J et al. (1999) Coping styles in animals: current status in behavior and stress-physiology Neuroscience \& Biobehavioral Reviews 23:925-935

Koolhaas JM, De Boer SF, Buwalda B, Van Reenen K (2007) Individual variation in coping with stress: a multidimensional approach of ultimate and proximate mechanisms Brain Behav Evol 70:218-226

Lecorps B, Kappel S, Weary DM, von Keyserlingk MA (2018) Dairy calves' personality traits predict social proximity and response to an emotional challenge Scientific reports 8:16350

Lee C, Cafe LM, Robinson SL, Doyle RE, Lea JM, Small AH, Colditz IG (2018) Anxiety influences attention bias but not flight speed and crush score in beef cattle Appl Anim Behav Sci 205:210-215

Lee C, Verbeek E, Doyle R, Bateson M (2016) Attention bias to threat indicates anxiety differences in sheep Biology letters 12:20150977 
Leliveld LM, Düpjan S, Tuchscherer A, Puppe B (2017) Vocal correlates of emotional reactivity within and across contexts in domestic pigs (Sus scrofa) Physiology \& behavior 181:117-126

Lukkes JL, Mokin MV, Scholl JL, Forster GL (2009) Adult rats exposed to earlylife social isolation exhibit increased anxiety and conditioned fear behavior, and altered hormonal stress responses Hormones and behavior 55:248-256

Marshall GN, Wortman CB, Kusulas JW, Hervig LK, Vickers Jr RR (1992) Distinguishing optimism from pessimism: Relations to fundamental dimensions of mood and personality Journal of personality and social psychology 62:1067

Melotti L, Oostindjer M, Bolhuis JE, Held S, Mendl M (2011) Coping personality type and environmental enrichment affect aggression at weaning in pigs Appl Anim Behav Sci 133:144-153

Mendl M, Burman OH, Parker RM, Paul ES (2009) Cognitive bias as an indicator of animal emotion and welfare: emerging evidence and underlying mechanisms Appl Anim Behav Sci 118:161-181

Mitchell EN, Marston HM, Nutt DJ, Robinson ES (2012) Evaluation of an operant successive negative contrast task as a method to study affective state in rodents Behavioural brain research 234:155-160

Monk JE, Belson S, Colditz IG, Lee C (2018a) Attention bias test differentiates anxiety and depression in sheep Frontiers in behavioral neuroscience 12

Monk JE, Doyle RE, Colditz IG, Belson S, Cronin GM, Lee C (2018b) Towards a more practical attention bias test to assess affective state in sheep PLoS One 13:e0190404

Munsterhjelm C, Peltoniemi OA, Heinonen M, Hälli O, Karhapää M, Valros A (2009a) Experience of moderate bedding affects behaviour of growing pigs Appl Anim Behav Sci 118:42-53

Munsterhjelm C, Peltoniemi OA, Heinonen M, Hälli O, Karhapää M, Valros A (2009b) Experience of moderate bedding affects behaviour of growing pigs Appl Anim Behav Sci 118:42-53

Munsterhjelm C, Valros A, Heinonen M, Hälli O, Siljander-Rasi H, Peltoniemi O (2010) Environmental enrichment in early life affects cortisol patterns in growing pigs animal 4:242-249

Paul ES, Harding EJ, Mendl M (2005) Measuring emotional processes in animals: the utility of a cognitive approach Neuroscience \& Biobehavioral Reviews 29:469-491 
Reimert I, Bolhuis JE, Kemp B, Rodenburg TB (2013a) Indicators of positive and negative emotions and emotional contagion in pigs Physiology \& behavior 109:42-50

Reimert I, Bolhuis JE, Kemp B, Rodenburg TB (2014a) Social support in pigs with different coping styles Physiology \& behavior 129:221-229

Reimert I, Rodenburg T, Ursinus W, Duijvesteijn N, Camerlink I, Kemp B, Bolhuis J (2013b) Backtest and novelty behavior of female and castrated male piglets, with diverging social breeding values for growth Journal of animal science 91:4589-4597

Reimert I, Rodenburg TB, Ursinus WW, Kemp B, Bolhuis JE (2014b) Responses to novel situations of female and castrated male pigs with divergent social breeding values and different backtest classifications in barren and straw-enriched housing Appl Anim Behav Sci 151:24-35

Roelofs S, Boleij H, Nordquist RE, van der Staay FJ (2016) Making decisions under ambiguity: judgment bias tasks for assessing emotional state in animals Frontiers in behavioral neuroscience 10:119

Ruis MA, te Brake JH, Engel B, Buist WG, Blokhuis HJ, Koolhaas JM (2001) Adaptation to social isolation: acute and long-term stress responses of growing gilts with different coping characteristics Physiology \& behavior 73:541-551

Sanger ME, Doyle RE, Hinch GN, Lee C (2011) Sheep exhibit a positive judgement bias and stress-induced hyperthermia following shearing Appl Anim Behav Sci 131:94-103

Segerstrom SC (2001) Optimism and attentional bias for negative and positive stimuli Personality and Social Psychology Bulletin 27:13341343

Sheriff MJ, Krebs CJ, Boonstra R (2009) The sensitive hare: sublethal effects of predator stress on reproduction in snowshoe hares Journal of Animal Ecology 78:1249-1258

Sipos ML, Bar-Haim Y, Abend R, Adler AB, Bliese PD (2014) Postdeployment threat-related attention bias interacts with combat exposure to account for PTSD and anxiety symptoms in soldiers Depression and Anxiety 31:124-129

Telkänranta H, Edwards SA (2017) Lifetime consequences of the early physical and social environment of piglets. In: Advances in Pig Welfare. Elsevier, pp 101-136

Ursin H, Olff M (1995) Aggression, defense, and coping in humans Aggressive Behavior 21:13-19 
Velie B, Maltecca C, Cassady J (2009) Genetic relationships among pig behavior, growth, backfat, and loin muscle area Journal of animal science 87:2767-2773

Verbeek E, Colditz I, Blache D, Lee C (2019) Chronic stress influences attentional and judgement bias and the activity of the HPA axis in sheep PLoS One 14:e0211363

Verbeek E, Ferguson D, de Monjour PQ, Lee C (2014) Generating positive affective states in sheep: The influence of food rewards and opioid administration Appl Anim Behav Sci 154:39-47

Winter KA, Kuiper NA (1997) Individual differences in the experience of emotions Clinical psychology review 17:791-821

Wright F, Rodgers R (2014) On the behavioural specificity of hypophagia induced in male rats by $\mathrm{MCPP}$, naltrexone, and their combination Psychopharmacology 231:787-800

Zebunke M, Repsilber D, Nürnberg G, Wittenburg D, Puppe B (2015) The backtest in pigs revisited-an analysis of intra-situational behaviour Appl Anim Behav Sci 


\section{Chapter 6}

Effects of early life and current housing on sensitivity to reward loss in a successive negative contrast test in pigs

L. Luo, I. Reimert, E.A.M Graat, S. Smeets, B. Kemp, J. E. Bolhuis* Adaptation Physiology Group, Department of Animal Sciences, Wageningen University \& Research, PO Box 338, 6700 AH, Wageningen, The Netherlands

*Corresponding author:

E-mail address: liesbeth.bolhuis@wur.nl

Published: Animal Cognition, 23(1), 121-130 


\subsection{Abstract}

Animals in a negative affective state seem to be more sensitive to reward loss, i.e. an unexpected decrease in reward size. The aim of this study was to investigate whether early-life and current enriched vs. barren housing conditions affect the sensitivity to reward loss in pigs using a successive negative contrast test. Pigs ( $n=64$ from 32 pens) were housed in barren or enriched conditions from birth onwards, and at 7 weeks of age experienced either a switch in housing conditions (from barren to enriched or vice versa) or not. Allotting pigs to the different treatments was balanced for coping style (proactive vs. reactive). One pig per pen was trained to run for a large reward and one for a small reward. Reward loss was introduced for pigs receiving the large reward after 11 days (reward downshift), i.e. from then onwards, they received the small reward. Pigs housed in barren conditions throughout life generally had a lower probability and higher latency to get the reward than other pigs. Proactive pigs ran overall slower than reactive pigs. After the reward downshift, all pigs ran slower. Nevertheless, reward downshift increased the latency and reduced the probability to get to the reward, but only in pigs exposed to barren conditions in early life, which thus were more sensitive to reward loss than pigs from enriched early life housing. In conclusion, barren housed pigs seemed overall less motivated for the reward, and early life housing conditions had long-term effects on the sensitivity to reward loss.

Key words: pigs, enrichment, early life, reward loss, coping style, affective state 


\subsection{Introduction}

The barren housing conditions in which most commercial pigs world-wide are housed limit the expression of important species-specific behaviours, like foraging and exploration (De Jonge et al. 1996; Studnitz et al. 2007; Wemelsfelder et al. 2000), and thereby increase the frequency of manipulative behaviours directed at pen mates, such as ear and tail biting (Beattie et al. 2000; Bolhuis et al. 2006; Carreras et al. 2016). Pigs in barren housing, moreover, show physiological signs of chronic stress (Beattie et al. 2000; Bolhuis et al. 2006; Carreras et al. 2016). This chronic stress could also be linked to a psychological state of (mild) depression. Indeed, pigs housed in barren conditions have been found to show a more pessimistic response in a judgement bias task, suggesting that they have a more negative affective state, compared with pigs in enriched conditions (Douglas et al. 2012), although in another study, no such effect of housing was found (Carreras et al. 2016).

A negative affective state may also enhance the sensitivity to reward loss (Chaby et al. 2013). For instance, people in a state of depression have been shown to be more susceptible to loss or failure (Tucker and Luu 2007). The response to reward loss can be measured in animals by a successive negative contrast (SNC) test (Burman et al. 2008; Flaherty et al. 1998). In a SNC test, reward loss is induced by unexpectedly decreasing the reward size or quality for animals that have been trained. This may induce a transient, potentially 'disappointment-like', aversive affective state, caused by the discrepancy between the anticipated reward, i.e. the reward the animals expected to receive, and the actual reward (Justel et al. 2014; Papini 2014; Rosas et al. 
2007). How aversively the animals respond to such a discrepancy (e.g., depending on the task, by reduced operant responses or a slower speed to get to the reward), has been suggested to be a sign of the animals' background, longer-term, affective state or mood (Flaherty et al. 1998; Mitchell et al. 2012; Riemer et al. 2016). In line with this, it has been shown that rats experiencing removal of enrichment in their home cages, thereby likely having a more negative affective state, responded stronger to a reward downshift, compared to control rats (Burman et al. 2008; Chaby et al. 2013). Increased sensitivity to reward loss appears thus to reflect negative affective states in humans and animals, and it could therefore be an useful measure of such states in pigs as well, which has, to the best of our knowledge, not been studied in this species so far.

In the current study, we do not only address the impact of housing conditions under which pigs are kept on their affective state, but also the influence of a barren vs. enriched environment in early life. It has been shown that adverse conditions in early life can have long-term effects on behaviour, physiology, and cognition. For example, isolation in early life decreases the motivation for social contact and sucrose-drinking in later life in rats (Van den Berg et al. 1999). Also in pigs, long-lasting effects of early life experiences have been found (Telkänranta and Edwards 2017). Importantly, pigs that changed from enriched housing in early life to barren housing later on show as much signs of decreased welfare as pigs kept in a barren environment throughout life, or even more (Bolhuis et al. 2004; Munsterhjelm et al. 2009), which suggests that a loss of enrichment in later life could be even more detrimental than barren housing throughout. In line with this, barren housed pigs tended to show more pessimistic judgement biases after they had temporarily 
experienced an enriched environment (Douglas et al. 2012). Thus, the impact of housing on affective states may not only depend on the actual housing conditions, but also on the conditions present in early life, and these two may interact.

The aim of this study was therefore to investigate the combined effects of early life environment and current housing conditions on the sensitivity to reward loss in pigs. Hereto, pigs housed in barren or enriched conditions in early life and experiencing either a switch in housing conditions at 7 weeks of age or not, were subjected to a SNC test at 12 weeks of age in which they had to run a U-shaped track to get to a food reward. Downsizing of the reward was expected to slow down the latency to get to the reward, indicative of an experience of reward loss. We hypothesized that barren housed pigs, and particularly those that had experienced enrichment in early life, would be more sensitive to reward loss, as they were expected to have a more negative affective state.

Personality traits may have an effect on behavioural responses in test situations, including negative contrast (Cuenya et al. 2012), attention bias (Franklin et al. 2016) and judgement bias tests (Asher et al. 2016). Therefore, pigs allotted to the different housing and rearing conditions in this study were balanced for their coping style, i.e. a personality trait related to the way an individual copes with challenging situations, as assessed in a backtest in early life (Hessing et al. 1994; Reimert et al. 2014b). 


\subsection{Materials and Methods}

The established principles of laboratory animal use and care were followed, as well as the Dutch law on animal experiments. The Animal Care and Use Committee of Wageningen University \& Research approved the experiment.

\subsubsection{Animals and housing}

In this experiment, 64 pigs (Tempo $\times$ Topigs 20) from 29 sows, equally divided over 2 batches were studied. Sows were inseminated on the same day in each batch, and were housed in Carus, the animal facilities of Wageningen University \& Research, Wageningen, the Netherlands, from one month before farrowing. From birth till weaning (around 28 days of age), half of the piglets within each batch were housed in $8.6 \mathrm{~m}^{2}$ barren (B1) pens with a solid floor and a small area with slats. The other half were housed in $17.1 \mathrm{~m}^{2}$ enriched (E1) pens with the same farrowing part $\left(8.6 \mathrm{~m}^{2}\right)$ as the barren pens, and with an additional enriched part. In the enriched part, $1.7 \mathrm{~kg}$ straw, 300 $\mathrm{L}$ sawdust, and $270 \mathrm{~L}$ peat were provided as substrates. Besides, $0.8 \mathrm{~kg}$ straw and $40 \mathrm{~L}$ of sawdust were added daily, and $30 \mathrm{~L}$ of peat was added weekly in the enriched pens. Additionally, two objects for manipulation, one chain with a ball and one chain with screws that touched the floor, were placed in the barren pens. Two objects, one chain with a ball and an object that was alternated daily and selected from 4 different ones, were placed in the enriched pens. All sows were housed in the same farrowing crates without access to the enrichment. In the first week after birth, one heating lamp was provided in the barren pens, and two lamps in the enriched pens. Each pen had one drinking nipple for the piglets and one for the sow. Sows were fed a standard commercial diet twice a day. From 5 days of age onwards, piglets 
received some creep feed. Temperature was set at $25{ }^{\circ} \mathrm{C}$ at birth, and gradually decreased to $21{ }^{\circ} \mathrm{C}$ over the course of two weeks. Each pen was cleaned daily, and lights and a radio were on from 7:00 until 19:00 hours.

At an average of 28 days of age, pigs were weaned and 192 pigs ( 96 per batch) were selected and regrouped in 32 new pens, which contained 6 healthy nonlittermate pigs (from the same pre-weaning treatment) each. Per group, 3 males and 3 females from different litters were selected, balanced for coping style ( $3 \mathrm{HR}$ and $3 \mathrm{LR}$, assessed and classified as described in Reimert et al. 2014a). Pigs fulfilling the criteria with a body weight closest to the litter mean weight were preferably selected so that the average body weight of the postweaning selection was representative of that of the whole group at weaning. Housing treatment (B1 vs. E1) for each pig was kept the same as before weaning. After weaning, half of the pigs were housed in $5.6 \mathrm{~m}^{2}$ barren pens, with a partly solid and slatted floor. The other half of the pigs were housed in $11.2 \mathrm{~m}^{2}$ pens enriched with $2.5 \mathrm{~kg}$ straw, $400 \mathrm{~L}$ of sawdust, and $360 \mathrm{~L}$ of peat on the floor. Additionally, $1.25 \mathrm{~kg}$ straw and $60 \mathrm{~L}$ sawdust were added daily, and $45 \mathrm{~L}$ peat was added weekly in the enriched pens. The toys in the barren and enriched pens were kept the same as before weaning, and from 39 days of age, enriched housed pigs received extra enrichment such as a jute sack, a rope, branches or an egg tray on each Monday until the end of the experiment (day 133).

Each pen had one drinking nipple and pigs received solid food ad libitum. On the weaning day, the temperature was set at $25{ }^{\circ} \mathrm{C}$ and it was gradually decreased to $21{ }^{\circ} \mathrm{C}$ over the course of two weeks and kept until the end of the experiment. After weaning, one heating lamp was provided in each pen 
for the duration of two weeks. Lights and a radio were on from 7:00 until 19:00 hours.

At an average of 47 days of age, half of the pigs experienced a switch in housing conditions, resulting in four treatment groups, E1E2, E1B2, B1E2, $B 1 B 2, n=8$ pens per group. For this switch, they were moved to a different pen. B1B2 and E1E2 groups were also removed to new pens, but without a change in housing condition. After this switch, straw, peat and toys were used and added in amounts as described before, but only $30 \mathrm{~L}$ of sawdust was added daily in the enriched pens.

\subsubsection{Successive negative contrast test}

To assess the sensitivity for reward loss as a measure of affective state, a successive negative contrast (SNC) test was applied from 84 to 120 days of age. Two female pigs per pen (one LR and one HR), in total 64 pigs, were selected from the 32 pens. These 64 pigs were 60 females and 4 males (of the 64 female pigs we initially selected, four had health issues. We replaced those with males from the same pen having the same coping style from the beginning of the test. All 4 males were from different pens, and 1 from B1B2, 2 from E1B2, and 1 from B1E2 housing conditions). Before the test period started, all pigs received pieces of apple in their home pen to accustom them to the reward in the test. Pieces of apple have been successfully used as a reward in previous research, and it has been shown that pigs prefer multiple pieces of apple (4, large reward) over a single piece (Melotti et al. 2013). Pigs were food deprived for at least one hour before the test, and allowed to eat after the two pigs in a pen finished the test. 
The test area was in a separate room away from the home pens. Pigs were habituated to the test arena in three days with 3 trials per day, initially in pairs (trial 1 on day 1 ), thereafter individually with a pen mate waiting in the start box (trial 2 and 3 on day 1), and finally individually without the presence of a pen mate (day 2 and 3). After this habituation period, pigs were individually allowed to run from a start box down a U-shaped runway (Figure 6.1 ) to obtain a food reward from a round plastic plate (diameter $32 \mathrm{~cm}$ ) at the end. One pig per pen received 6 pieces of apple (1 piece was $1 / 48$ part of an apple (Elstar variety, without the core) as the large reward on each trial, and the other one received 1 piece of apple as the small reward. Even though all pigs ate apple in their home pen, some pigs $(n=6)$ did not eat apple in the test and were given banana instead ( 1 piece was $1 / 4$ of a $1 \mathrm{~cm}$ thick slice) after the habituation days. After habituation, pigs were allowed to run for the food reward on four days per week. Each pig did 3 trials per day, with a maximum of $120 \mathrm{sec}$ per trial. A trial was finished when the pig ate the reward within the $120 \mathrm{sec}$, or when the pig turned back five times (i.e. started walking in a direction not leading to the reward) or, in case the pig had not finished eating the reward within the maximum time set, at $120 \mathrm{sec}$. During each trial, the latency to leave the start box (i.e. the pig was in the runway with all 4 legs) and the latency to start eating were recorded. From these two latencies the latency to reward was calculated by subtracting the latency leaving the start box from the latency to start eating. If a pig did not start eating the reward within the $120 \mathrm{sec}$, the latency to reward was set to 120 sec.

Pigs were excluded from further testing if they reached the maximum time (120 sec) and did not reach the reward on three consecutive test days ( $n=7$ 
pigs), if they reached the reward but did not eat it on three consecutive test days ( $n=4$ pigs), or if they encountered health problems (umbilical hernia: $n=1$, lameness: $n=1$ ). In this experiment, 51 pigs ( 48 females and 3 males, and 6 pigs with banana as reward) were successfully trained and included in the analyses. Within one batch, all pigs were tested on the same days.

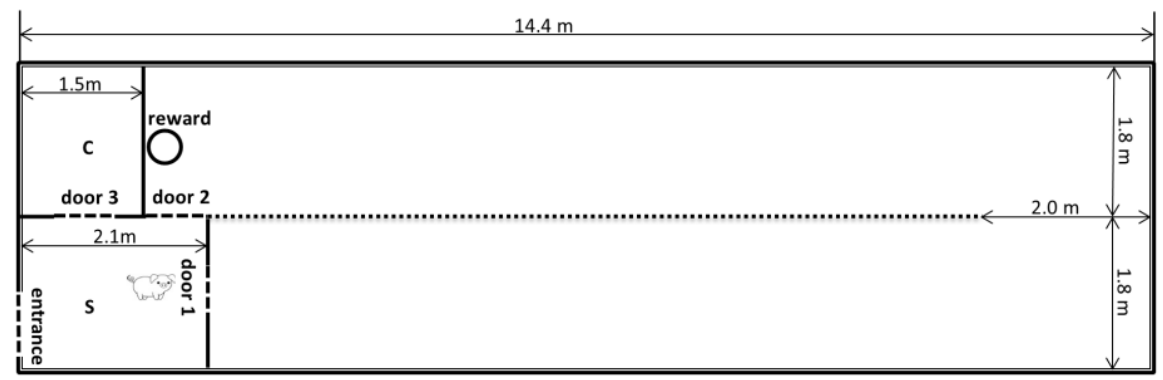

Figure 6.1: Layout of the test area used for the successive negative contrast test. The area consisted of a U-shaped runway. The two arms of the runway $(12.3 \times 1.8 \mathrm{~m}$ and $12.9 \times 1.8 \mathrm{~m}$ ) were separated by a wired fence (height: $1.2 \mathrm{~m}$ ). Pigs were brought in a start box (S), and entered the runway from door 1. Pigs could run in the U-shaped runway to get the reward in a plastic plate (diameter $32 \mathrm{~cm}$ ) screwed on the floor. After pigs finished a trial, they left the runway from door 2, and waited in the start box for another trial, until all 3 trials were finished. Two experimenters stayed in a compartment (C) to operate the doors and record the latencies.

Reward downshift

After 11 days, all pigs received a small reward only on another 11 subsequent days (reward downshift, creating a 6-1 vs. 1-1 reward group), i.e. the pigs originally receiving 6 pieces of apple or banana experienced a reward loss. We planned to proceed to the reward downshift when the pigs in the large reward group would run significantly faster (Burman et al. 2008) than the 
pigs in the small reward group, but set a maximum of 11 pre-reward downshift days (Burman et al. 2008) to minimize pigs from losing interest in the test. After 11 training days, not all (housing treatment groups of) pigs that received the large reward ran significantly faster than the pigs that received the small reward, therefore the reward downshift started at day 12 and ended on day 22.

\subsubsection{Statistical analyses}

SAS (SAS 9.4, SAS Institute Inc.) was used for all statistical analyses.

Censoring occurred for pigs that failed to get the reward within $120 \mathrm{sec}$. Therefore, survival analysis was used for the latency to reward. First, KaplanMeier survivor functions were estimated. Subsequently, Cox proportional hazard regression was performed with animal as a random effect to estimate the hazard ratios with $95 \%$ confidence interval. The explanatory variables early life housing (pre-housing: B1, E1), current housing (post-housing: B2, E2), original reward size $(1,6)$, reward downshift (from day 12 onwards, all 6-1 pigs were "YES"; all 1-1 pigs were "NO"), coping style (HR, LR), trial number $(1,2,3)$, test day $(1,2, \ldots, 22)$ and batch $(1,2)$ were analysed as class variables. A multivariable Cox regression analysis was started with all the variables above and their two- and three-way interactions. Subsequently, a stepwise backward selection procedure was performed deleting nonsignificant variables starting with the highest $p$-value, until all factors in the model had $p<0.05$. The variables pre-housing, post-housing and original reward size remained in the model, even if they were not significant, as these variables were included in the main research aims in this study. Therefore, the final multivariable model consisted of the variables pre-housing, post- 
housing and their interaction, reward downshift and the interaction of reward downshift with pre-housing, original reward size, coping style, test day and trial number and the random effect of pig.

It was checked whether the proportional hazard assumption was not violated, i.e. the hazard functions of groups are proportional over time. If a hazard ratio (HR) is smaller than 1 , then the probability to eat the reward is smaller compared to the reference class, and if it is larger than 1 the probability is higher. If the value 1 is included in the $95 \%$ confidence interval, then there is no difference between groups.

\subsection{Results}

In total, 3355 records could be used based on 51 pigs on 22 test days, with 3 trials per day per pig.

The probability for trials in which pigs from each housing treatment got the reward at some point in time is illustrated in a Kaplan-Meier curve (Figure $6.2, \log$-rank $p<0.0001)$. The percentage of censored records, i.e. trials in which pigs did not get the reward within the maximum time of $120 \mathrm{sec}$ was higher for the B1B2 pigs (43.9\%), compared to B1E2 (17.0\%), E1B2 (17.4\%), and E1E2 (22.5\%) pigs. 


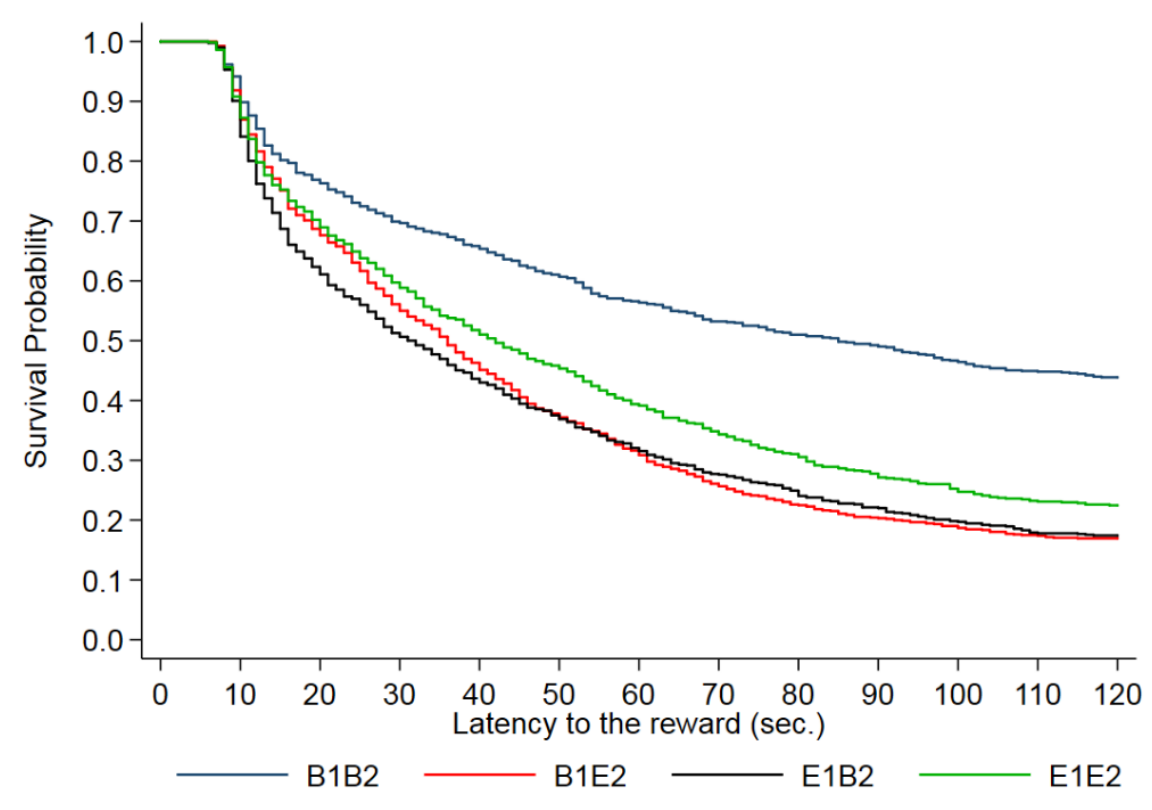

Figure 6.2: Kaplan-Meier curve showing the latency to reward for pigs from each housing group, with a maximum trial duration of $120 \mathrm{sec}$. For each trial in which a pig got to the reward, the probability on the $Y$-axis drops. B1B2 and E1E2 refer to pigs housed in barren and enriched pens, respectively, throughout the experiment; B1E2 and E1B2 refer to pigs that experienced a change in environment from barren to enriched or vice versa from 7 weeks of age.

The multivariate model showed that the effect of current housing on latency to the reward depended on the early life housing (pre-housing $\times$ posthousing interaction, $p=0.001$ ), or vice versa. Pigs that had switched from barren to enriched housing (B1E2) had a higher probability of getting the reward and lower latency to the reward compared to B1B2 pigs housed in barren conditions throughout life ( $\mathrm{HR}=2.48, p<0.0001$, Figure 6.3), however, for the pigs housed in an enriched environment in early life there was no 
effect of later life housing (E1B2 vs. E1E2). For barren housed pigs in later life (B2), E1 pigs had a higher probability of getting the reward and a lower latency than B1 pigs, both for no reward downshift (HR $=3.61, p<0.0001)$ and for reward downshift ( $H R=2.32, p=0.0005)$. When pigs had enriched housing later in life (E2), there was no effect of pre-housing.

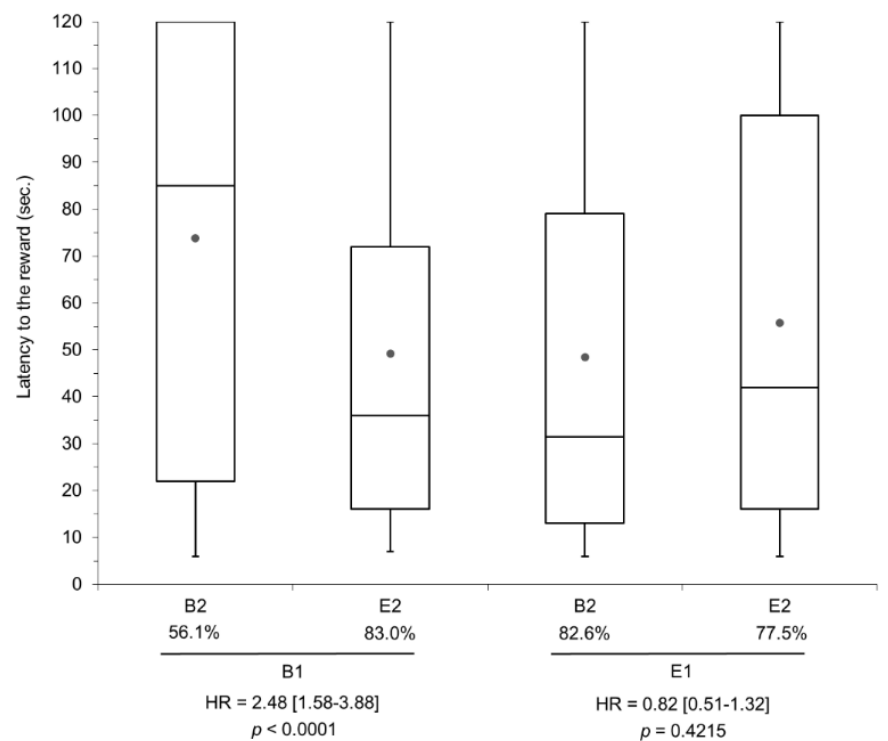

Figure 6.3: Box-Whisker plot for the latency to the reward with the percentage of total trials to get to the reward $(\bullet=$ mean), and the stratum-specific hazard ratio (HR) with $95 \%$ confidence interval for the pre-housing $\times$ post-housing interaction $(p<0.05)$. $B 1$ and $E 1$ refer to barren and enriched housing in early life, respectively, and $B 2$ and E2 refer to barren and enriched housing from 7 weeks of age onwards. Note that latency to the reward is underestimated because the observations were censored at $120 \mathrm{sec}$. 
Latency to the reward was not different between pigs from the large reward group (6-1: $72.8 \%, 57.1 \pm 1.2 \mathrm{sec})$ compared to the pigs that always received a small reward (1-1: 76.2\%, $56.0 \pm 1.0 \mathrm{sec}, \mathrm{HR}=1.09, p=0.6228)$ in the whole test period, including the 11 days before reward downshift. Pigs that experienced a reward downshift had a lower probability to get to the reward and a higher latency to the reward, but this effect was only significant for pigs housed in barren conditions in early life (HR $=0.58, p<0.0001$, Figure 6.4 ) to reflect the interaction between pre-housing and reward downshift ( $p$ $=0.0004)$.

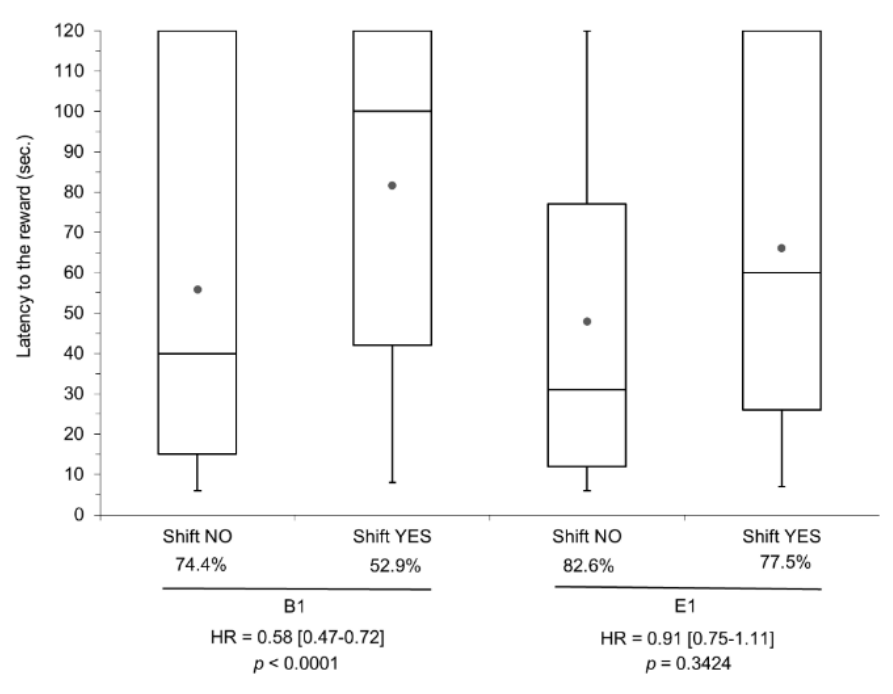

Figure 6.4: Box-Whisker plot for the latency to the reward with the percentage of total trials to get to the reward, and the stratum-specific Hazard Ratio with 95\% confidence interval for the pre-housing $\times$ reward downshift (YES or NO) interaction ( $p$ $=0.0004)$. B1 and E1 refer to barren and enriched housing in early life, respectively. 
Note that latency to the reward is underestimated because the observations were censored at $120 \mathrm{sec}$.

Figure 6.5a presents the mean and median time of latency to the reward over test days, as well as the percentage of trials in which pigs got to the reward. A test day effect was found $(p<0.0001$, Figure 6.5). At the first 6 test days, the probability to get to the reward was higher compared to day 12 (the day of reward downshift), being significant for day 2 to day 6 ( $p<0.05$ or less, Figure $6.5 \mathrm{~b})$. The probability on test day 7 to 11 was not different from day 12. After reward downshift, the probability to get to the reward decreased and was on all days lower compared to day 12 (Figure 6.5b).

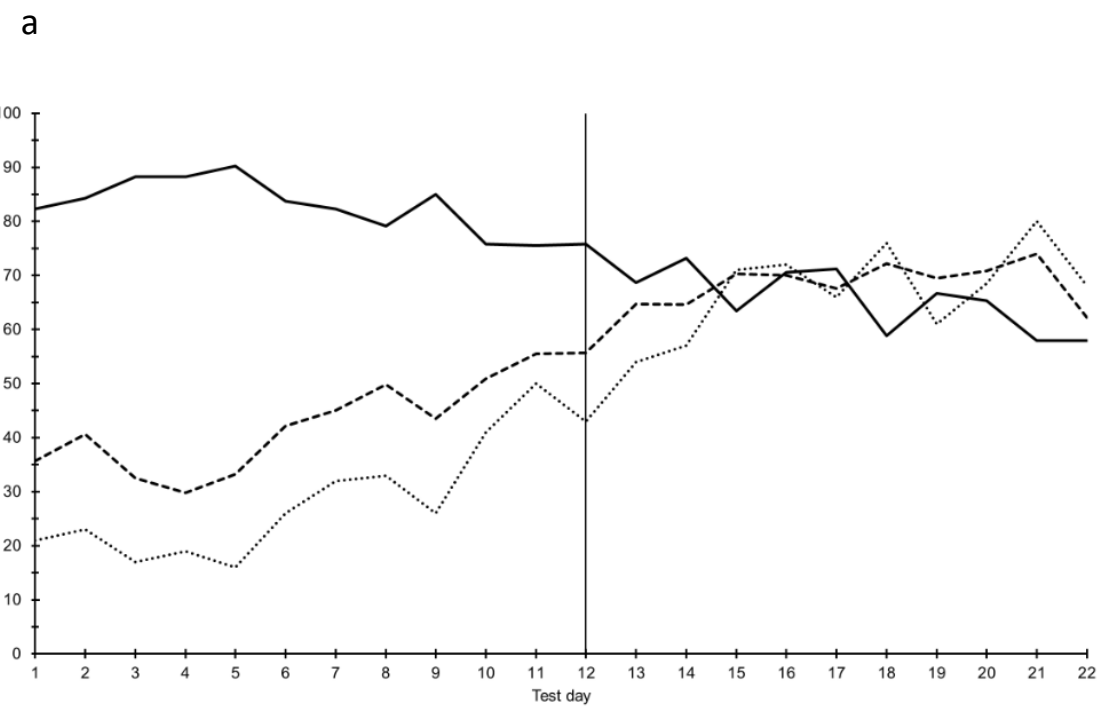


b

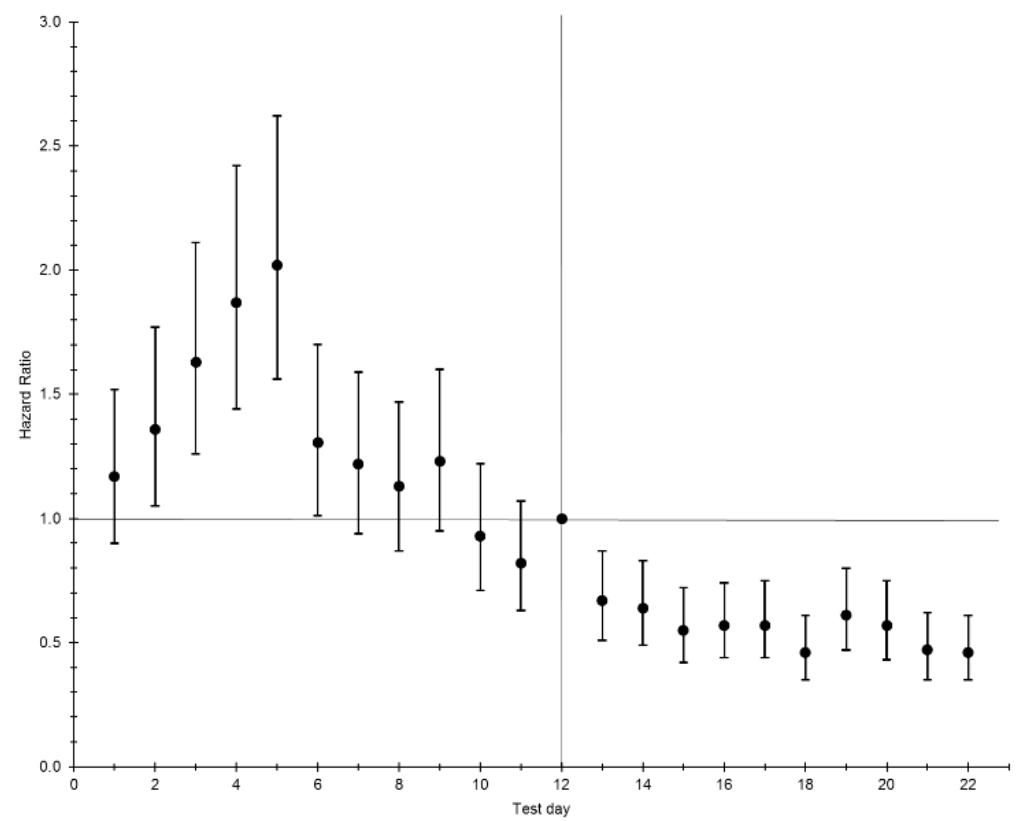

Figure 6.5: Panel a shows the mean and median time of the latency to the reward and the percentage of total trials in which pigs got to the reward within $120 \mathrm{sec}$ for each test day. Note that means and median times are underestimated because the observations were censored at 120 sec. Panel b shows hazard ratios with 95\% confidence intervals per test day. If the value 1.00 is within the confidence interval, the hazard ratio is not significantly different from day 12, the day of reward downshift (overall test day effect $p<0.0001$ ).

Pigs had a lower probability to get to the reward and higher latency to the reward in trial $2(69.9 \%, 62.7 \pm 1.3 \mathrm{sec}, \mathrm{HR}=0.40, p<0.0001)$ and trial $3(66.1$ $\pm 1.2 \mathrm{sec}, 70.3 \%, \mathrm{HR}=0.35, p<0.0001)$, compared with trial $1(84.1 \%, 39.8 \pm$ $1.2 \mathrm{sec}$ ) within a test day. 
Low-resisters $(78.7 \%, 52.2 \pm 1.0 \mathrm{sec})$ had a higher probability of getting the reward and lower latency to the reward compared to high-resisters $(71.0 \%$, $60.1 \pm 1.0 \mathrm{sec}, \mathrm{HR}=1.48, p=0.0187)$.

\subsection{Discussion}

In this study, we aimed to investigate the effect of early and later life housing conditions on the sensitivity to reward loss in pigs in a successive negative contrast test (SNC) using a runway. In the whole test period, both before and after the reward downshift and irrespective of reward size, pigs that were housed barren throughout life had a lower probability and a higher latency to get to the reward than pigs from the other three housing combinations, i.e. enrichment throughout life, enrichment in early life only, or barren housing in early life followed by enrichment. Early life housing affected the sensitivity to reward loss, as only in pigs from early life barren housing (B1E2 and B1B2) an effect of the reward downshift was found. Coping style also affected latency to the reward, with reactive pigs having a higher probability and a shorter latency to get to the reward than proactive pigs.

\subsubsection{Effects of reward loss}

Pigs ran faster from day 2 to day 6, compared with day 12 when the reward was downshifted. After the reward downshift, all pigs, including pigs that did and did not experience a reward downshift, ran slower than before the reward downshift and probability to get to the reward reduced. It could be that pigs reduced their interest in the test and reward over time, which is possibly also reflected by the higher latency to the reward in the second and 
third trial on a test day as compared with the first trial. Alternatively, they may have become slower due to the increase in body weight over test days, as at this age, the pigs gained approximately $1.1 \mathrm{~kg}$ per day.

Nevertheless, there was an effect of reward downshift on the latency and probability to the reward, but this effect was only significant in pigs from early life barren housing (see below). When an anticipated food reward is unexpectedly reduced, many mammals show a decreased response to the reward, compared to controls which received only the lower level reward (Bergvall et al. 2007; Catanese et al. 2011). The decrease in reward size may lead to disappointment-like or frustration-like affective responses, which can influence the motivation for the reward (Burman et al. 2008). Thus, even though pigs generally decreased their running speed over time, the reward reduction was still aversive for some of them, and caused a successive negative contrast effect in pigs from barren early life housing.

Burman et al. (2008) reported a higher sensitivity to reward loss in rats that experienced a loss of enrichment in their housing environment. In this study, we found that the pigs exposed to barren housing in early life (B1B2 and B1E2) were more sensitive to reward loss, as only in these animals the running speed and probability to get to the reward was affected by the reward downshift. This may suggest a long-term effect of a poor environment on the (negative) affective state in later life.

We expected the highest sensitivity for reward loss in the pigs from early life enriched housing that switched to barren pens in later life, as results of several studies indicate that a loss of enrichment may be more detrimental than no experience with enrichment at all (Beattie et al. 1995; Bolhuis et al. 
2006; Douglas et al. 2012). Such an effect was not found, however, as the latency to the reward for E1B2 pigs did not differ from the latency for E1E2 pigs. The reason could be that the appraisal of the test conditions may have interfered with the effects we intended to study, as being (trained) in a test such as a runway may be rewarding in itself and provide cognitive enrichment, thereby inducing a temporary positive affective state. If so, this may have blunted or overruled (early life) housing effects on long-term mood (Bethell et al. 2016; Roelofs et al. 2016). Apart from potential rewarding properties of the test itself, the finding that early life enrichment makes pigs more resilient to reward loss, might counteract a potential negative effect of the reduction in environmental quality. Alternatively, results of a recent study suggest that previous exposure to an experience of frustration, which, in our study, could be the case for pigs that switched from enriched to barren pens, may counteract the effects of a new frustrating situation, like SNC (Cuenya et al. 2012). Further research is needed to test such an effect in pigs.

\subsubsection{Motivation to run for the reward}

We found that B1B2 pigs, irrespective of reward size, had lower probability and higher latency to get to the reward than the other pigs, both before as well as after the reward downshift, which may show that pigs kept in barren housing conditions throughout life have a lower motivation to run for the reward. Diminished sensitivity to reward can be interpreted as a characteristic of poor mood or anhedonia (Von Frijtag et al. 2000). Anhedonia is the reduced reactivity to pleasurable stimuli or positive effects from events or activities, and is one of the core symptoms of depression (Bevins and Besheer 2005; Leppänen 2006; Von Frijtag et al. 2000). Indeed, 
it has been found that chronic stress caused by tail handling led to a lower response to reward in mice (Clarkson et al. 2018) and juvenile isolation reduced motivation for sucrose drinking in rats (Van den Berg et al. 1999). As a depression-like state or chronic stress has also been linked with barren housing conditions (Beattie et al. 2000; Douglas et al. 2012), the lower probability to get to the reward in the B1B2 pigs in this study may have reflected anhedonia and a low reward sensitivity. On the other hand, some adverse conditions have also been found to increase the sensitivity to reward (Van den Berg et al. 1999). For example, acute stress caused by isolation increased the motivation for food in hens (Hernandez et al. 2015), and chronic mild stress in rats and music inducing a depressed mood in humans increased the motivation for highly positive sweet food rewards, albeit the latter can also be interpreted as a measure of craving rather than response to the reward (Willner et al. 1998). The relationship between mood and reward sensitivity is thus not that straightforward. Moreover, other cognitive studies where pigs had to run to find a food reward as well reported no (Bolhuis et al. 2013; Bolhuis et al. 2004) or only limited (Grimberg-Henrici et al. 2016) evidence for a lower motivation for rewards in pigs when housed barren. Another, alternative, explanation of the lower probability to get to the reward in B1B2 pigs could be that they had spent more time on exploring (parts of) the runway, to 'catch up' from the limited space and stimuli in their housing environment. It should be noted, though, that this effect was not seen in barren housed pigs that had been exposed to an enriched environment in early life.

This is, to the best of our knowledge, the first SNC test in pigs. Over pre-shift days, latencies to get to the reward were higher in the last 5 test days. Also, 
not all pigs that were initially trained were motivated to run for apple pieces, even though apples have been successfully used as a reward in another study which, in addition, demonstrated that pigs prefer 4 pieces over 1 piece of apple (Melotti et al. 2013). Motivation could possibly be enlarged in future studies by a longer food deprivation before testing, restricted feeding or by determining the animals' individual preference for a particular reward before the experiment (Zebunke et al. 2018). It could also be that the pigs in this study lost their interest in the task in general over trials and over test days, so the optimal testing period and number of trials per day need to be considered in future studies.

We did not find a difference in response to the small vs. large reward before the reward downshift, in contrast with a study on rats using 12 vs. 1 food pellets (Burman et al. 2008). However, in another rat study with a similar 1:12 approach (Cuenya et al. 2012), no pre-shift difference in latency to reward was found between the small and large reward group either, and, similar to our study, still an SNC effect could be demonstrated. Unlike previous studies in rats (Burman et al. 2008; Cuenya et al. 2012; Pellegrini et al. 2004) and dogs (Bentosela et al. 2009) which demonstrated a return to pre-shift responses after 5-6 post-shift days, we found no signs of a recovery in running speed in the pigs that experienced a reward loss, as there was no interaction between day and reward group. It should be noted that, even though statistically significant, the effect of reward loss was mild compared to rat studies in which latencies roughly doubled in animals experiencing a reward downshift (Burman et al. 2008; Cuenya et al. 2012). In these rat studies, the ratio between the large and small reward was larger (1:12), and, 
moreover, the large reward was a substantial part of their daily portion of feed.

Our main interest was to evaluate the effect of rearing and housing conditions on reward loss sensitivity in pigs. As personality traits of animals have an effect on their behavioural responses, including those in tests for affective state (Cuenya et al. 2012; Franklin et al. 2016), we characterized all pigs early in life by the backtest as 'high-resisters' or 'low-resisters' (Bolhuis et al. 2004; Hessing et al. 1994; Reimert et al. 2014a). The response of pigs in this backtest, which is heritable (Iversen et al. 2017; Velie et al. 2009; Zebunke et al. 2015), reflects their tendency to adopt a proactive (also called active) or reactive (also called passive) coping response (Bolhuis et al. 2005; Koolhaas 2001; Koolhaas et al. 1999). To account for a potential coping style influence, allocation of pigs to the housing treatments and reward groups was balanced for backtest classification, which was subsequently added as a fixed effect in the analyses. There was an effect on the latency to reward, as low-resisters had a lower latency to get to the reward than high-resisters. It is difficult to say whether this effect was due to a difference in how lowresisters and high-resisters valued the reward and were motivated for the task, or reflected a difference in balance between different motivations (e.g. to explore the runway vs. go for the reward immediately). Concerning the latter, the shorter latencies of the low-resister pigs are not in line with general findings that these pigs explore for longer, even in a familiar test room (Jansen et al. 2009). Nonetheless, it is important for future research to keep in mind that personality traits, such as coping style, may affect important read out parameters of tests of affective state. The number of animals included in the task did not allow us to test all potential interactions 
between coping style with the four rearing and housing combinations and the two reward sizes. For future studies, however, it could be interesting to further investigate potential interactions between environmental conditions and personality, which have been found for judgement bias (Asher et al. 2016), behaviour in the home pen (Bolhuis et al. 2005; Bolhuis et al. 2006) and behaviour in a T-maze (Bolhuis et al. 2004), on the sensitivity to reward loss.

\subsection{Conclusions}

Barren housed pigs and pigs with a proactive coping personality had a longer latency to get to the reward throughout the test which could either reflect their affective state, or, alternatively, a difference in balance between the motivation to explore the test area and the motivation for the reward. Irrespective of their current housing conditions, pigs originating from a barren pen in early life responded to the reward loss, as reflected in a reduced probability and increased latency to get to the reward following reward downshift, whereas such an effect was not found in pigs originating from enriched pens. This may indicate that negative early life experiences may have a long-term impact on the ability of pigs to cope with reward loss and on affective state. We found no clear evidence for an effect of current housing, nor for an effect of a change in housing (from barren to enriched or vice versa) on reward loss sensitivity. 


\subsection{Acknowledgements}

The authors would like to thank M. van Marwijk, M. Ooms, E. N. de Haas, and B. Laurenssen for skilful assistance in conducting the experiment. We are also grateful to the animal caretakers and students involved.

\subsection{Compliance with ethical standards}

6.9 Conflict of interest The authors declare that they have no conflict of interest.

6.10 Ethical approval All applicable institutional guidelines for the care and use of animals were followed. All procedures performed in studies involving animals were in accordance with the ethical standards of the institution or practice at which the studies were conducted. 


\subsection{References}

Asher L, Friel M, Griffin K, Collins LM (2016) Mood and personality interact to determine cognitive biases in pigs Biology letters 12:20160402

Beattie V, O'Connell N, Kilpatrick D, Moss B (2000) Influence of environmental enrichment on welfare-related behavioural and physiological parameters in growing pigs Animal Science 70:443-450

Beattie V, Walker N, Sneddon I (1995) Effect of rearing environment and change of environment on the behaviour of gilts Appl Anim Behav Sci 46:57-65

Bentosela M, Jakovcevic A, Elgier AM, Mustaca AE, Papini MR (2009) Incentive contrast in domestic dogs (Canis familiaris) J Comp Psychol 123:125

Bergvall UA, Rautio P, Luotola T, Leimar O (2007) A test of simultaneous and successive negative contrast in fallow deer foraging behaviour Anim Behav 74:395-402

Bethell EJ, Holmes A, MacLarnon A, Semple S (2016) Emotion evaluation and response slowing in a non-human primate: New directions for cognitive bias measures of animal emotion? Behavioral Sciences 6:2

Bevins RA, Besheer J (2005) Novelty reward as a measure of anhedonia Neuroscience \& Biobehavioral Reviews 29:707-714

Bolhuis JE, Oostindjer M, Hoeks CW, de Haas EN, Bartels AC, Ooms M, Kemp B (2013) Working and reference memory of pigs (Sus scrofa domesticus) in a holeboard spatial discrimination task: the influence of environmental enrichment Anim Cogn 16:845-850

Bolhuis JE, Schouten WG, de Leeuw JA, Schrama JW, Wiegant VM (2004) Individual coping characteristics, rearing conditions and behavioural flexibility in pigs Behavioural brain research 152:351-360

Bolhuis JE, Schouten WG, Schrama JW, Wiegant VM (2005) Individual coping characteristics, aggressiveness and fighting strategies in pigs Anim Behav 69:1085-1091

Bolhuis JE, Schouten WG, Schrama JW, Wiegant VM (2006) Effects of rearing and housing environment on behaviour and performance of pigs with different coping characteristics Appl Anim Behav Sci 101:68-85

Burman OH, Parker RM, Paul ES, Mendl M (2008) Sensitivity to reward loss as an indicator of animal emotion and welfare Biology Letters 4:330333 
Carreras R et al. (2016) Housing conditions do not alter cognitive bias but affect serum cortisol, qualitative behaviour assessment and wounds on the carcass in pigs Appl Anim Behav Sci 185:39-44

Catanese F, Freidin E, Cuello MI, Distel RA (2011) Devaluation of low-quality food during early experience by sheep Animal 5:938-942

Chaby L, Cavigelli S, White A, Wang K, Braithwaite V (2013) Long-term changes in cognitive bias and coping response as a result of chronic unpredictable stress during adolescence Frontiers in human neuroscience 7:328

Clarkson JM, Dwyer DM, Flecknell PA, Leach MC, Rowe C (2018) Handling method alters the hedonic value of reward in laboratory mice Scientific reports 8:2448

Cuenya $L$ et al. (2012) The effect of partial reinforcement on instrumental successive negative contrast in inbred Roman High-(RHA-I) and Low(RLA-I) Avoidance rats Physiology \& behavior 105:1112-1116

De Jonge FH, Bokkers E, Schouten W, Helmond F (1996) Rearing piglets in a poor environment: developmental aspects of social stress in pigs Physiology \& behavior 60:389-396

Douglas C, Bateson M, Walsh C, Bédué A, Edwards SA (2012) Environmental enrichment induces optimistic cognitive biases in pigs Appl Anim Behav Sci 139:65-73

Flaherty CF, Greenwood A, Martin J, Leszczuk M (1998) Relationship of negative contrast to animal models of fear and anxiety Anim Learn Behav 26:397-407

Franklin ZC, Holmes PS, Smith NC, Fowler NE (2016) Personality Type Influences Attentional Bias in Individuals with Chronic Back Pain PLoS One 11:e0147035

Grimberg-Henrici CG, Vermaak P, Bolhuis JE, Nordquist RE, van der Staay FJ (2016) Effects of environmental enrichment on cognitive performance of pigs in a spatial holeboard discrimination task Anim Cogn 19:271-283

Hernandez CE, Hinch G, Lea J, Ferguson D, Lee C (2015) Acute stress enhances sensitivity to a highly attractive food reward without affecting judgement bias in laying hens Appl Anim Behav Sci 163:135-143

Hessing MJ, Hagels $\varnothing$ AM, Schouten WG, Wiepkema PR, Van Beek JA (1994) Individual behavioral and physiological strategies in pigs Physiology \& Behavior 55:39-46 
Iversen M, Bolhuis J, Camerlink I, Ursinus W, Reimert I, Duijvesteijn N (2017) Heritability of the backtest response in piglets and its genetic correlations with production traits animal 11:556-563

Jansen J, Bolhuis JE, Schouten WG, Spruijt BM, Wiegant VM (2009) Spatial learning in pigs: effects of environmental enrichment and individual characteristics on behaviour and performance Anim Cogn 12:303315

Justel N, Pautassi RM, Psyrdellis M, Mustaca A (2014) Mediational role of hormones in incentive contrast International Journal of Comparative Psychology 27

Koolhaas J (2001) How and why coping systems vary among individuals Coping with Challenge

Koolhaas J et al. (1999) Coping styles in animals: current status in behavior and stress-physiology Neuroscience \& Biobehavioral Reviews 23:925-935

Leppänen JM (2006) Emotional information processing in mood disorders: a review of behavioral and neuroimaging findings Current opinion in psychiatry 19:34-39

Melotti L, Thomsen LR, Toscano MJ, Mendl M, Held S (2013) Delay discounting task in pigs reveals response strategies related to dopamine metabolite Physiology \& behavior 120:182-192

Mitchell EN, Marston HM, Nutt DJ, Robinson ES (2012) Evaluation of an operant successive negative contrast task as a method to study affective state in rodents Behavioural brain research 234:155-160

Munsterhjelm C, Peltoniemi OA, Heinonen M, Hälli O, Karhapää M, Valros A (2009) Experience of moderate bedding affects behaviour of growing pigs Appl Anim Behav Sci 118:42-53

Papini MR (2014) Diversity of adjustments to reward downshifts in vertebrates International Journal of Comparative Psychology 27

Pellegrini S, Muzio RN, Mustaca AE, Papini MR (2004) Successive negative contrast after partial reinforcement in the consummatory behavior of rats Learning and Motivation 35:303-321

Reimert I, Rodenburg TB, Ursinus WW, Kemp B, Bolhuis JE (2014a) Responses to novel situations of female and castrated male pigs with divergent social breeding values and different backtest classifications in barren and straw-enriched housing Appl Anim Behav Sci 151:24-35

Reimert I, Rodenburg TB, Ursinus WW, Kemp B, Bolhuis JE (2014b) Selection based on indirect genetic effects for growth, environmental 
enrichment and coping style affect the immune status of pigs PLoS One 9:e108700

Riemer S, Ellis SL, Ryan S, Thompson H, Burman OH (2016) A reappraisal of successive negative contrast in two populations of domestic dogs Anim Cogn 19:471-481

Roelofs S, Boleij H, Nordquist RE, van der Staay FJ (2016) Making decisions under ambiguity: judgment bias tasks for assessing emotional state in animals Frontiers in behavioral neuroscience 10:119

Rosas JM et al. (2007) Successive negative contrast effect in instrumental runway behaviour: a study with Roman high-(RHA) and Roman low(RLA) avoidance rats Behavioural Brain Research 185:1-8

Studnitz M, Jensen MB, Pedersen LJ (2007) Why do pigs root and in what will they root?: A review on the exploratory behaviour of pigs in relation to environmental enrichment Appl Anim Behav Sci 107:183-197

Telkänranta H, Edwards SA (2017) Lifetime consequences of the early physical and social environment of piglets. In: Advances in Pig Welfare. Elsevier, pp 101-136

Tucker DM, Luu P (2007) Neurophysiology of motivated learning: adaptive mechanisms underlying cognitive bias in depression Cognitive Therapy and Research 31:189-209

Van den Berg CL, Pijlman FT, Koning HA, Diergaarde L, Van Ree JM, Spruijt BM (1999) Isolation changes the incentive value of sucrose and social behaviour in juvenile and adult rats Behavioural Brain Research 106:133-142

Velie B, Maltecca C, Cassady J (2009) Genetic relationships among pig behavior, growth, backfat, and loin muscle area Journal of animal science 87:2767-2773

Von Frijtag J, Reijmers L, Van der Harst J, Leus I, Van den Bos R, Spruijt B (2000) Defeat followed by individual housing results in long-term impaired reward-and cognition-related behaviours in rats Behavioural brain research 117:137-146

Wemelsfelder F, Haskell M, Mendl MT, Calvert S, Lawrence AB (2000) Diversity of behaviour during novel object tests is reduced in pigs housed in substrate-impoverished conditions Anim Behav 60:385394

Willner P, Benton D, Brown E, Cheeta S, Davies G, Morgan J, Morgan M (1998) "Depression" increases "craving" for sweet rewards in animal and human models of depression and craving Psychopharmacology $136: 272-283$ 
Zebunke M, Kreiser M, Melzer N, Langbein J, Puppe B (2018) Better, Not Just More-Contrast in Qualitative Aspects of Reward Facilitates Impulse Control in Pigs Frontiers in psychology 9

Zebunke M, Repsilber D, Nürnberg G, Wittenburg D, Puppe B (2015) The backtest in pigs revisited-an analysis of intra-situational behaviour Appl Anim Behav Sci 


\section{Chapter 7}

\section{Early and later life environmental enrichment affect specific antibody responses and blood leukocyte subpopulations in pigs}

L. Luo ${ }^{1}$, C. A. Jansen ${ }^{2}$, J. E. Bolhuis ${ }^{1}$, J. A. J. Arts ${ }^{1}$, B. Kemp ${ }^{1}$, H. K.

$$
\text { Parmentier }^{1^{*}}
$$

${ }^{1}$ Adaptation Physiology Group, Department of Animal Sciences, Wageningen University \& Research, $6700 \mathrm{AH}$, Wageningen, the Netherlands

${ }^{2}$ Department of Infectious Diseases and Immunology, Faculty of Veterinary Medicine, Utrecht University, 3508 TD, Utrecht, the Netherlands

*Correspondence: H. K. Parmentier

E-mail address: $\underline{\text { henk.parmentier@wur.nl }}$ 


\subsection{Abstract}

This study addressed the impact of early and later life environmental enrichment, and their combination, on specific antibody responses and peripheral blood leukocyte subpopulations in pigs. Pigs were kept in either barren (B1) or enriched (E1) housing from birth, and half of the pigs switched to barren or enriched housing on day 47 , resulting in four treatment combinations: B1B2, B1E2, E1B2, E1E2). Pigs were immunized with keyhole limpet hemocyanin-conjugated trinitrophenyl (KLH-TNP) on day 74 and 109 to induce primary and secondary antibody responses. Blood samples were taken weekly until day 130, and IgM and IgG antibody responses were measured. Leukocyte subpopulations were measured on day 74 and 130. Time course of the antibody responses was not affected by housing. Early life enrichment increased the IgG response to KLH, particularly the primary one. At day 74 the relative frequency of lymphocytes, DC and SLA-II expression on monocytes were higher in E1 pigs, whereas the percentage of granulocytes tended to be lower in E1 pigs at day 74. Early life enrichment increased the SLA-II expression on monocytes, the granulocyte to lymphocyte ratio, and tended to increase the percentage of granulocytes, but tended to decrease the percentage of monocytes at day 130. Later life enrichment reduced percentages of $C D 4+C D 8 \alpha^{+} T$ cells before and after immunization and the SLA-II expression on monocytes at day 74 , the percentage of granulocytes and the granulocyte to lymphocyte ratio at day 130 . Notably, early and later life housing interacted in their effects on several immune parameters. KLHIgM responses (both primary and secondary) were affected by the interaction between early and later life housing. IgM titers were higher for B1B2 than for E1E2, with the switched animals (B1E2 and E1B2) moving 
towards the titers of the animals kept in their later life environment from birth onwards. At day 130 the percentage of gamma delta T cells, CD8 $\alpha^{+}$ cytotoxic T cells and DC were not different between pigs kept in B1B2 and E1E2, but there was a clear impact of the switch in housing conditions, particularly for the pigs that changed from barren to enriched housing. We also found effects of coping style (personality) and sex on some immune parameters. In conclusion, both early and later life enrichment, and, notably a switch in housing conditions influenced specific antibodies and leukocyte subpopulations in pigs. The current study implies that the early life history of animals and the (mis)match with their current environment could thus be of major importance for their immune system. Further research is needed to investigate potential consequences for the pigs' health.

Key words: pigs; enrichment; early life history; coping style; immunity; antibody response 


\subsection{Introduction}

Pigs in commercial farms may experience chronic stress caused by the barren housing environment in which they are usually kept. Limited living space, and the lack of materials to forage prevent pigs from expressing natural behaviours, leading to damaging behaviours like biting the tails and ears of pen mates (Beattie et al. 2000), cognitive impairment (Bolhuis et al. 2013; Grimberg-Henrici et al. 2016), and negative emotions (Bolhuis et al. 2005; Douglas et al. 2012). Stress can result in altered innate (Cui et al. 2016) and adaptive (Bacou et al. 2017) immune functions, and also positive and negative emotions can trigger immune alterations (Dhabhar 2014; Koh 1998). Environmental enrichment is defined as an increase of the biological relevance of captive environments by appropriate modifications (such as more space and, in the case of pigs, the provision of rooting substrates) resulting in an improvement of the biological functioning of captive animals (Newberry 1995). Enriched environments, as opposed to barren housing, have been shown to reduce damaging behaviours, induce a more positive emotional (affective) state (Douglas et al. 2012), and influence the levels of natural (auto)antibodies (Luo et al. 2017a; Luo et al. 2017b; Reimert et al. 2014b) and viral clearance in pigs (van Dixhoorn et al. 2016). We recently found that enriched housed pigs had higher levels of natural IgM antibodies binding myelin basic protein (MBP), a neural antigen, and tended to have higher levels of natural IgG binding MBP (Luo et al. 2017b). Besides, the effect of infections with porcine reproductive and respiratory syndrome virus (PRRSV) and Actinobacillus pleuropneumoniae on natural (auto)antibodies was influenced by housing in pigs in either barren or enriched conditions from birth (Luo et al. 2017a). 
Apart from the current housing environment of pigs, however, early life conditions may also have profound effects on later performance. It has been shown in humans that stressful early life experiences may lead to increased vulnerability to immune dysregulation later in life (see Elwenspoek et al. 2017; Fagundes et al. 2013 for review) and, in rats, maternal separation in early life was found to increase plasma corticosterone and the systemic immune response after an in vitro lipopolysaccharide challenge (O'Mahony et al. 2009). There are indications that also in pigs' early life experiences affect immune status. Lewis et al. (Lewis et al. 2012) showed that pigs isolated in early life had more $\mathrm{CD} 4^{+}$and $\mathrm{CD} 4^{+} \mathrm{CD} 25^{+}$effector T-cell staining in the intestinal mucosa, and a reduced $\mathrm{CD}^{+} \mathrm{CD} 25^{+} \mathrm{Foxp}^{+}$regulatory $\mathrm{T}$-cell population at weaning, compared to pigs reared with sows, resulting in a higher T-reg-to-effector ratio in the latter animals. This suggests that the early life environment may profoundly affect local development of regulatory components of the mucosal immune system. Schokker et al. (Schokker et al. 2015; Schokker et al. 2014) showed that exposure to stress in early life affected gut microbial colonization and intestinal immune development, although it should be noted that effects of antibiotic administration at day 4 of life were more pronounced. Although, to our knowledge, no data are available on the specific impact of early life enrichment on systemic immune competence of pigs in later life, evidence has been presented for long-term effects of being raised in a spacious, stimulus-rich environment on their social skills (De Jonge et al. 1996) and sensitivity to reward loss (Luo et al. under review). It can be hypothesized that the differences induced by early life enrichment may exert long-term effects on the pigs' immune system as well. Moreover, the potential impact 
of early life enrichment on immunity may depend on the housing conditions of pigs in later life, which may or may not match with the way they were reared. This is of relevance given that commercial pigs usually experience at least one move (but often more) to a different pen, room, and sometimes farm, in their lives. Although a handful of studies indicate that the effects of current housing on behaviour and welfare of pigs partly depend on early life conditions (and vice versa) (e.g. Bolhuis et al. 2005; Douglas et al. 2012; Munsterhjelm et al. 2009), whether such an interaction also holds for immune parameters, is, to the best of our knowledge, unknown.

We therefore aimed to investigate the effect of early and later life enrichment and the combination of those on 1) specific primary and secondary antibody responses and 2) the relative frequencies of peripheral blood leukocyte subpopulations in pigs to assess their immune status. Treatment groups were balanced for sex and coping style, a personality trait, which affected immune parameters in other studies (Bolhuis et al. 2003; Kanitz et al. 2019; Luo et al. 2017b; Oster et al. 2015; Reimert et al. 2014b).

\subsection{Material and methods}

Established principles of laboratory animal use and care and the Dutch law on animal experiments were followed. The Animal Care and Use Committee of Wageningen University approved the experiment.

Figure 7.1 gives an outline of the experimental set-up. Briefly (for details see below), pigs were raised in either barren or enriched housing conditions from birth. At 47 days of age, they experienced either a switch in housing (from 
barren to enriched or vice versa), or remained in their original housing type (barren or enriched). Thus, four treatment groups were formed in a $2 \times 2$ arrangement, with early life and later life (post-switch) housing as factors using $n=64$ pigs from 32 pens in total. At 74 and 119 days of age, all pigs were immunized with the same antigen to mount a primary and secondary antibody response.

\subsubsection{Animals and housing}

Piglets (Tempo $\times$ Topigs 20) originated from 27 litters, divided over 2 batches. Pigs were not castrated, tail docked or teeth clipped. From birth till weaning, half of the piglets were housed with sow in $8.6 \mathrm{~m}^{2}$ barren (B) pens with solid floor, and the other half in $17.1 \mathrm{~m}^{2}$ enriched $(E)$ pens with the same $B$ part and an additional enriched part with $1.7 \mathrm{~kg}$ of straw, $300 \mathrm{~L}$ of sawdust, and $270 \mathrm{~L}$ of peat. Daily, $0.8 \mathrm{~kg}$ straw and $40 \mathrm{~L}$ of sawdust were added, and weekly $30 \mathrm{~L}$ peat. B pens contained 2 toys, one chain with a ball and one with screws. E pens contained one permanent toy (a chain with a ball) and one toy selected from 4 different toys that rotated daily. All sows were housed in farrowing crates in the B part. In the first week after birth, heating lamps were provided. Each pen had drinking nipples for pigs and sows. Sows were fed a standard diet twice a day, and piglets received fresh feed from day 5. Temperature was $25^{\circ} \mathrm{C}$, and gradually decreased to $21^{\circ} \mathrm{C}$.

At 13 days of age, piglets were subjected to a backtest to assess their coping style, also referred to as personality (Bolhuis et al. 2000). Briefly, in this test, piglets are restrained in supine position for $1 \mathrm{~min}$ and the number and latency of escape attempts and vocalisations are recorded (see (Melotti et al. 
2011) for details). Pigs were either classified as "high resisters" (HR) or "lowresisters" (LR) as described before (Reimert et al. 2014b).

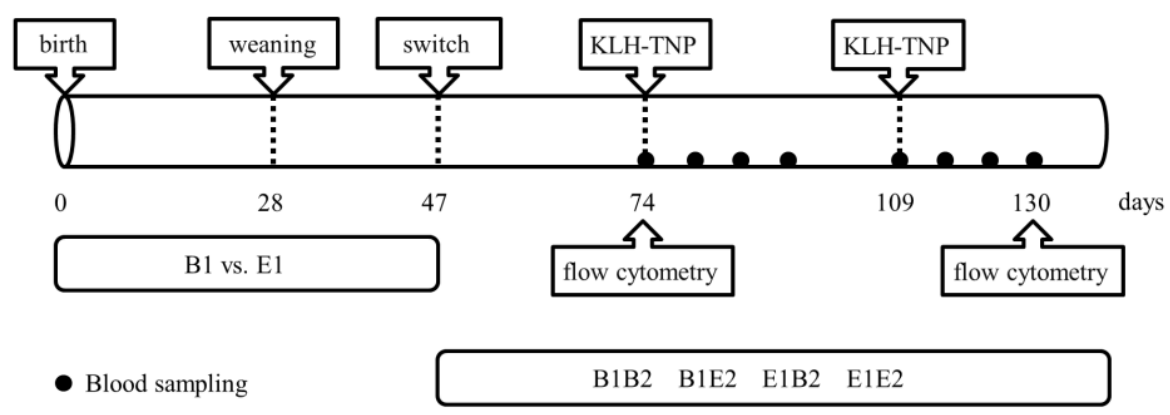

Figure 7.1: Experimental set-up. Pigs were housed in either barren (B1) or enriched (E1) condition from birth till 47 days of age, when half of pigs experienced a housing switch, creating B1B2, B1E2, E1B2, and E1E2 housing conditions. Two pigs per pen were immunized with KLH-TNP on 74 and 109 days of age, and blood samples were taken to measure antibody and leukocytes.

\subsubsection{Weaning, regrouping and switch}

Pigs were weaned at on average 28 days of age, and their housing treatment (B vs. E) was kept the same as before. In each of 32 pens, 6 unfamiliar pigs were grouped, balanced for sex (1:1) and coping style ( 3 HR and 3 LR) to minimize between-pen variation, as these two factors may influence immune parameters. Taking into account these criteria, piglets with body weights close to the mean of their litter were selected. Selection of the two pigs per pen to be immunized followed the same criteria. B pigs were moved to 5.6 $\mathrm{m}^{2} \mathrm{~B}$ pens, with partly solid and partly slatted floor. E pigs were moved to $11.2 \mathrm{~m}^{2} \mathrm{E}$ pens with $2.5 \mathrm{~kg}$ straw, $400 \mathrm{~L}$ of sawdust, and $360 \mathrm{~L}$ of peat. Daily, $1.25 \mathrm{~kg}$ straw and $60 \mathrm{~L}$ sawdust were added, and weekly $45 \mathrm{~L}$ peat. Toys were same as before. From 39 days of age, E pigs received extra enrichment (toys 
or substrates) weekly. The housing conditions before the switch are labelled with a "1" (i.e. B1 or E1), which means the early life housing experience, in the text referred to as "pre-housing".

Pens had one drinking nipple and pigs received solid food ad libitum. One heating lamp was provided for two weeks. Pens were cleaned daily and lights and radio were on from 7:00 until 19:00. Temperature was $25^{\circ} \mathrm{C}$ at weaning and gradually decreased to $21^{\circ} \mathrm{C}$.

Half of the $E$ and $B$ groups were allocated to switch conditions at 47 days of age, and the other half not, resulting in treatment groups B1B2, E1B2, B1E2, and E1E2. For the switch, each group of pigs was moved to a different pen, including the B1B2 and E1E2 groups, without a change in enrichment conditions. Pigs remained in the same group. Substrates were used and added as before, but only $30 \mathrm{~L}$ of sawdust was given daily to $\mathrm{E}$ pigs. Conditions after the switch are referred to as "post-housing". Part of the pigs within a pen were exposed to tests of emotional state and all of them were observed to study their behaviour in the home pens. These results are presented elsewhere (Luo et al. 2019a; Luo et al. 2019b) (Luo et al., submitted).

\subsubsection{Immunization and blood sampling}

Per pen, two pigs (balanced for sex and coping style within treatment), n=16 per treatment, i.e. in total 64 pigs were primary immunized subcutaneously with $1 \mathrm{mg} / \mathrm{ml}$ keyhole limpet hemocyanin-conjugated trinitrophenyl (KLHTNP, LGC Biosearch Technologies, Petaluma, CA, USA) in $1 \mathrm{ml}$ PBS at 74 days of age, and secondary immunized with the same dose of antigen at 109 days of age was done. Blood samples were collected (order balanced for treatment) in heparin tubes (Greiner Bio-one, Alphen aan den Rijn, The 
Netherlands) on 74, 81, 88, 95, and 109 days of age (i.e. 0, 1, 2, 3 and 5 weeks after first immunization), and on 116,123, and 130 days of age (i.e. 1, 2, and 3 weeks after second immunization). Blood was kept on ice, and centrifuged at $5251 \times \mathrm{g}$ for $10 \mathrm{~min}$ at $4{ }^{\circ} \mathrm{C}$. Plasma was stored at $-20{ }^{\circ} \mathrm{C}$ until analysis. In addition, whole blood samples were collected at 74 and 130 days of age (before and after immunization) in the same heparin tubes and kept at RT for flow cytometric analysis of immune cell subsets.

\subsubsection{Enzyme-linked immunosorbent assay}

To study specific IgM and IgG responses to KLH-TNP, and KLH separately, specific IgM and IgG antibody titers binding KLH-TNP (LGC Biosearch Technologies) and KLH (Sigma-Aldrich, St. Louis, MO, USA) were determined by a two-step indirect enzyme-linked immunosorbent assay (ELISA). Medium-binding microtiter plates (Greiner Bio-One) were coated overnight at $4{ }^{\circ} \mathrm{C}$ with $4 \mu \mathrm{g} / \mathrm{ml} \mathrm{KLH}$-TNP or $4 \mu \mathrm{g} / \mathrm{ml} \mathrm{KLH}$ in coating buffer $\left(5.3 \mathrm{~g} / \mathrm{L} \mathrm{Na}_{2} \mathrm{CO}_{3}\right.$ $+4.2 \mathrm{~g} / \mathrm{L} \mathrm{NaHCO}_{3}, \mathrm{pH}$ 9.6). Plates were washed with tap water containing $0.05 \%$ Tween 20, and tapped to remove the excess of washing buffer. Based on earlier tests plasma was prediluted $1 / 50$ and $1 / 25$ for IgM and IgG binding KLH-TNP and KLH respectively. Samples were 4-step wise serially diluted in PBS in plates and incubated for $1.5 \mathrm{~h}$ at RT. After washing, plates were incubated for $1.5 \mathrm{~h}$ at RT with a 1:40000 diluted goat-anti-swine-IgM-HRP (GASwIgM/PO, Bethyl Laboratories, Montgomery, TX, USA) to detect binding of $\operatorname{lgM}$, or with a 1:20000 diluted goat-anti-swine $\lg G_{F C} H R P\left(G A S w \mid g G_{F C} / P O\right.$, Bethy Laboratories) to detect binding of IgG to KLH-TNP and KLH, respectively. After washing, tetramethylbenzidine (TMB) was added as a substrate for 10 min. Reaction was stopped with $1.25 \mathrm{M} \mathrm{N} \mathrm{H}_{2} \mathrm{SO}_{4}$ and absorbance was 
measured at $450 \mathrm{~nm}$ with a Multiskan Go (Thermo scientific, Breda, the Netherlands). Absorbance was expressed relative to that of a standard positive control sample, and antibody titers were expressed as $\log _{2}$ values of dilutions that gave extinction closest to $50 \%$ of $E_{\max }$, where $E_{\max }$ represents the highest mean extinction of a standard positive sample present on every microtiter plate. All laboratory analyses were performed blind to the treatment (also for flow cytometry).

\subsubsection{Flow cytometry}

Flow cytometry was performed to estimate the frequencies of leukocyte subpopulations in the blood before and after immunization. Twenty-five microliters of whole blood were transferred to a $96 \mathrm{U}$ bottom Deepwell plate (Thermo Fisher Scientific, Waltham, USA) and stained with several combinations of monoclonal antibodies. The antibody panels are shown in Table 7.1. T-cell subsets and NK cells were stained with $\operatorname{mix} 1$, a combination of mouse-anti-pig CD4-FITC (clone 74-12-4, IgG2b, Southernbiotech, Birmingham, USA), mouse-anti-pig CD8 $\alpha-P E$ (clone 76-2-11; IgG2a, Southernbiotech), mouse-anti-pig CD3ع-PeCy7 (clone BB23-8E6-8C8, IgG2a, BD biosciences, San Jose, USA) and rat-anti-pig $\gamma \delta$-APC (clone MAC320, IgG2a,BD Biosciences). B-cells and antigen presenting cells were stained with mix 2, a combination of mouse-anti-pig CD21-PE (clone BB6-11C9.6, IgG1, Southernbiotech), mouse-anti-pig CD14-FITC (clone MIL2, IgG2b Bio-Rad, Puchheim, Germany), mouse-anti-pig pan-myeloid cell marker SWC3/CD172a-biotin (clone 74- 22-15A, IgG1, Southernbiotech) and an unconjugated mouse-anti-pig-SLA Class II DR antibody (clone 2E9/13, IgG2b, Bio-Rad). Cells were stained for $20 \mathrm{~min}$ at RT. Next, erythrocytes were lysed 
using lysis buffer (BD biosciences). After $15 \mathrm{~min}$ at RT cells were centrifuged for $5 \mathrm{~min}$ at $1300 \mathrm{rpm}$ and the pellet was resuspended in PBS supplemented with $0.5 \%$ BSA. Next, a combination of streptavidin-PercP (BD biosciences) and goat-anti-mouse-IgG2b APC (Southernbiotech) was added to the samples stained with mix 2 or to samples without a primary antibody mix as negative control. Cells were stained for $20 \mathrm{~min}$ at $4^{\circ} \mathrm{C}$, washed in PBS supplemented with $0.5 \%$ BSA and flow cytometry was performed using a FACS Canto flow cytometer (BD Biosciences). At least 100,000 lymphocytes were collected. Data were analysed using FlowJo software (Threestar Inc, San Carlo, USA).

Table 7.1: Monoclonal antibodies used for flow cytometry.

\begin{tabular}{|c|c|c|c|c|}
\hline Antibody name & Panel & Isotype & Clone & Target \\
\hline mouse-anti-pig CD4 FITC & $\operatorname{mix} 1$ & $\operatorname{lgG} 2 b$ & $74-12-4$ & CD4+ T helper c€ \\
\hline mouse-anti-pig-CD $8 \alpha \mathrm{PE}$ & $\operatorname{mix} 1$ & $\operatorname{lgG} 2 a$ & $76-2-11$ & CD8+ cytotoxic $\mathrm{T}$ \\
\hline mouse-anti-pig $\mathrm{CD} 3 \varepsilon$ PeCy7 & $\operatorname{mix} 1$ & $\lg 2 a$ & BB23-8E6-8C8 & T cells \\
\hline rat-anti-pig $\gamma \delta$ APC & $\operatorname{mix} 1$ & $\lg G 2 a$ & clone MAC320 & gamma delta T c \\
\hline mouse-anti-pig CD21 PE & $\operatorname{mix} 2$ & $\operatorname{lgG1}$ & BB6-11C9.6 & B cells \\
\hline mouse-anti-pig CD14 FITC & $\operatorname{mix} 2$ & $\lg G 2 b$ & MIL2 & myeloid cells \\
\hline mouse-anti-pig SWC3/CD172a BIOTIN & $\operatorname{mix} 2$ & IgG1 & clone 74-22-15A & myeloid cells \\
\hline mouse-anti-pig-SLA Class II DR UNL & $\operatorname{mix} 2$ & $\lg G 2 b$ & $2 \mathrm{E} 9 / 13$ & SLA-II \\
\hline Streptavidin PercP & & - & - & biotin \\
\hline goat-anti-mouse-IgG2b APC & & - & - & $\lg G 2 a$ \\
\hline
\end{tabular}

Peripheral blood leukocyte subpopulations were analysed by flow cytometry. A representative example of the gating strategy to identify different subsets of innate and adaptive immune cells is shown in Figure 7.2 and Figure 7.3. Lymphocytes were selected based on scatter (Figure 7.2A). T cells ( $\mathrm{CD3}^{+}$cells) were gated and within this population several subsets including gamma delta $\mathrm{T}$ cells, $\mathrm{CD} 4^{+}$helper T cells, $\mathrm{CD} 8 \alpha^{+}$cytotoxic $\mathrm{T}$ cells and $\mathrm{CD} 4^{+} \mathrm{CD} 8 \alpha^{+} \mathrm{T}$ cells were 
analysed. Also, NK cells ( $\mathrm{CD} 3^{-} \mathrm{CD} 4^{-} \mathrm{CD} \alpha^{+}$cells) and $\mathrm{B}$ cells ( $\mathrm{CD} 21^{+}$cells) were studied. In Figure 7.3 the large cells were gated based on forward and side scatter. In Figure 7.3, based on SWC3/CD172a expression, two SWC3/CD172 $\mathrm{a}^{\text {hi }}$ subsets and a SWC3/CD172a ${ }^{\text {low }}$ subset were defined. CD172a is expressed on dendritic cells (DC) in blood (Summerfield et al. 2003). Granulocytes (Nguyen et al. 2016) and monocytes are also known to express $\mathrm{CD} 172 \mathrm{a}$, and CD172a expression on monocytes is higher compared to the expression DC (McCullough et al. 1997). Based on side scatter, SWC3/CD172a ${ }^{\text {hi }}$ cells can be further separated into monocytes and granulocytes (Nguyen et al. 2016). Based on literature and SLA-II expression we defined SWC3/CD172 $a^{\text {hisSClow }}$ cells as monocytes in the present study, SWC3/CD172a ${ }^{\text {hi }}$ SSC $^{\text {hi }}$ cells as granulocytes and SWC3/CD172a $a^{\text {low }}$ SSC $^{\text {low }}$ cells as DC. CD14 staining did not result in a clear population of positive cells in these pigs (data not shown) and was therefore not included in further analysis of innate immune cell subsets. Apart from the relative frequencies of leukocyte subsets identified, granulocyte to lymphocyte ratios were analysed as well. 
A

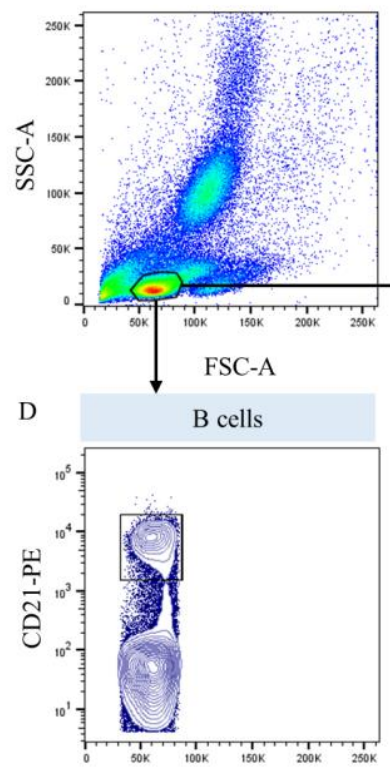

FSC-A
B

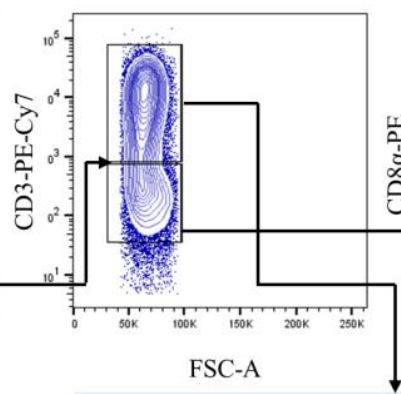

E

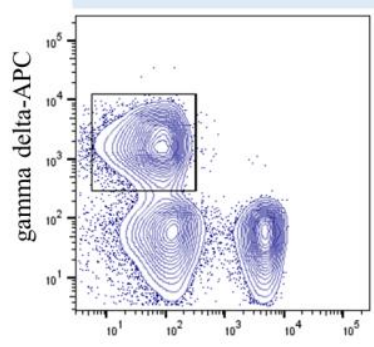

CD4-FITC
C NK cells

$\mathrm{T}$ cells

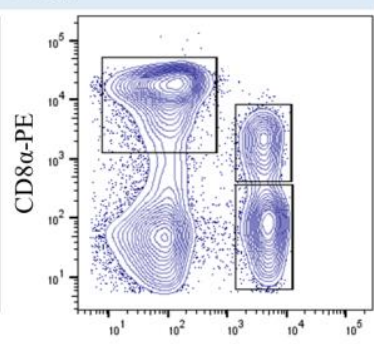

CD4-FITC

Figure 7.2: Gating strategy to identify lymphocyte subsets. A: Lymphocytes were gated based on forward and side scatter. B: Lymphocytes were divided into CD3- non $T$ cells and $\mathrm{CD}^{+} \mathrm{T}$ cells. C: Within the CD3- non T cell population, NK cells were defined as $C D 4^{-} C D 8 \alpha^{+}$cells. D: B cells were identified as $C D 21^{+}$cells within the lymphocyte population. E: $C D 3^{+} T$ cells were further separated into gamma delta $T$ cells, $C D 8 \alpha^{+} T$ cells, CD4 $4^{+} T$ cells and $C D 4^{+} C D 8 \alpha^{+} T$ cells. 


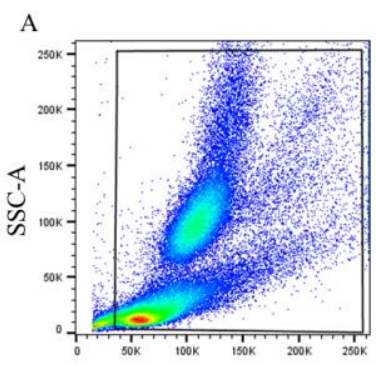

FSC-A

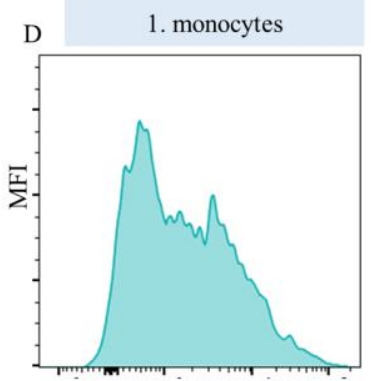

SLA-II DR-APC

G

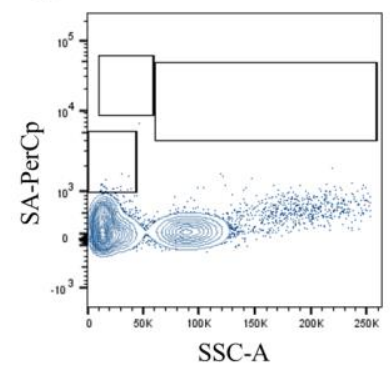

B

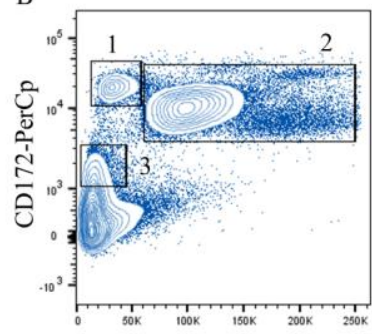

SSC-A

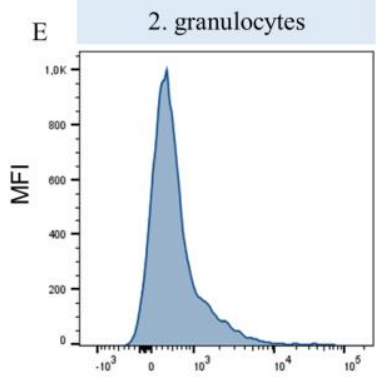

SLA-II DR-APC

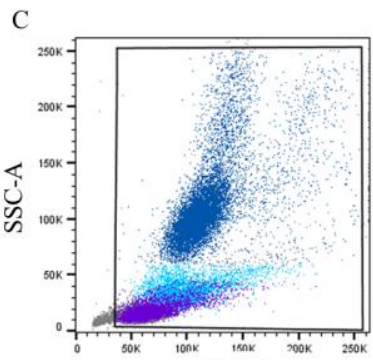

FSC-A

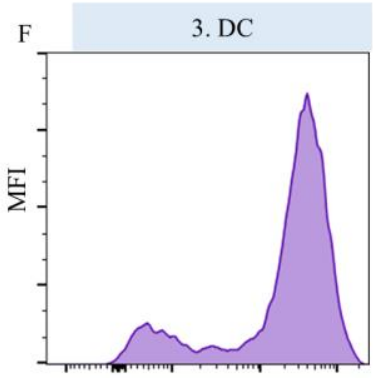

SLA-II DR-APC

Figure 7.3: Gating strategy to identify subsets of monocytes, granulocytes and dendritic cells (DC). A: Large cells were gated based on forward and side scatter. $B$ : Based on SWC3/CD172a expression and SSC this population was further separated into SWC3/CD172a $a^{\text {hi }}$ SSClow monocytes (population 1), SWC3/CD172a $a^{\text {hi }}$ SSC $C^{\text {hi }}$ granulocytes (population 2) and SWC3/CD172alow SSClow DC (population 3). C: Backgating of monocytes (light blue), granulocytes (dark blue) and DC (purple). Expression of SLA-II DR on monocytes $(D)$, granulocytes $(E)$ and $D C(F)$ was determined. G: The conjugate control shows cells stained with streptavidin-PercP only. 


\subsubsection{Statistical analyses}

SAS (SAS 9.4, SAS Institute Inc.) was used for all statistical analyses. Normality of error distribution, and homogeneity of variance were examined graphically, and based on this, the percentage of monocytes was logtransformed. Two pigs were euthanized (because of umbilical hernia and lameness).

Antibody titers were analysed using a repeated linear mixed model. Fixed effects of pre-housing (housing before the switch, B1 or E1), post-housing (housing after the switch, B2 or E2), sampling day, their interactions, batch, sex, and coping style (HR vs. LR) were included in the model. Values in time of individual animals were taken as repeated measurements. A linear model with pre-housing, post-housing, their interactions, batch, sex and coping style as fixed effects was used to analyse their influence on antibody titers before immunization (at 74 days of age) and on percentages of leukocyte subpopulations at 74 and 130 days of age.

Significant interactions $(p<0.05)$ were further investigated with post hoc pairwise comparisons using the difference of the least square means. If significant sampling day effects were found, pairwise comparisons between days were adjusted using Tukey corrections. Results are presented as means \pm SEM. 


\subsection{Results}

\subsubsection{Effects of housing conditions on antibody responses}

Specific IgM and IgG antibody responses to KLH and KLH-TNP were similar, indicating that the KLH-TNP antibody responses mainly reflected antibodies binding KLH (data not shown). Therefore, only responses to KLH are reported.

\subsubsection{KLH-IgM}

Before immunization. KLH-IgM titers before immunization at day 74 were affected by post-housing $\left(\mathrm{F}_{(1,57)}=6.47, \mathrm{p}=0.014\right)$; B2 pigs $(2.46 \pm 0.10)$ had higher titers than E2 pigs $(2.09 \pm 0.10)$. No effects of pre-housing $\left(F_{(1,57)}=0.93\right.$, $p=0.338)$ or the pre-housing $\times$ post-housing interaction $\left(F_{(1,57)}=0.07, p=0.793\right)$ were found.
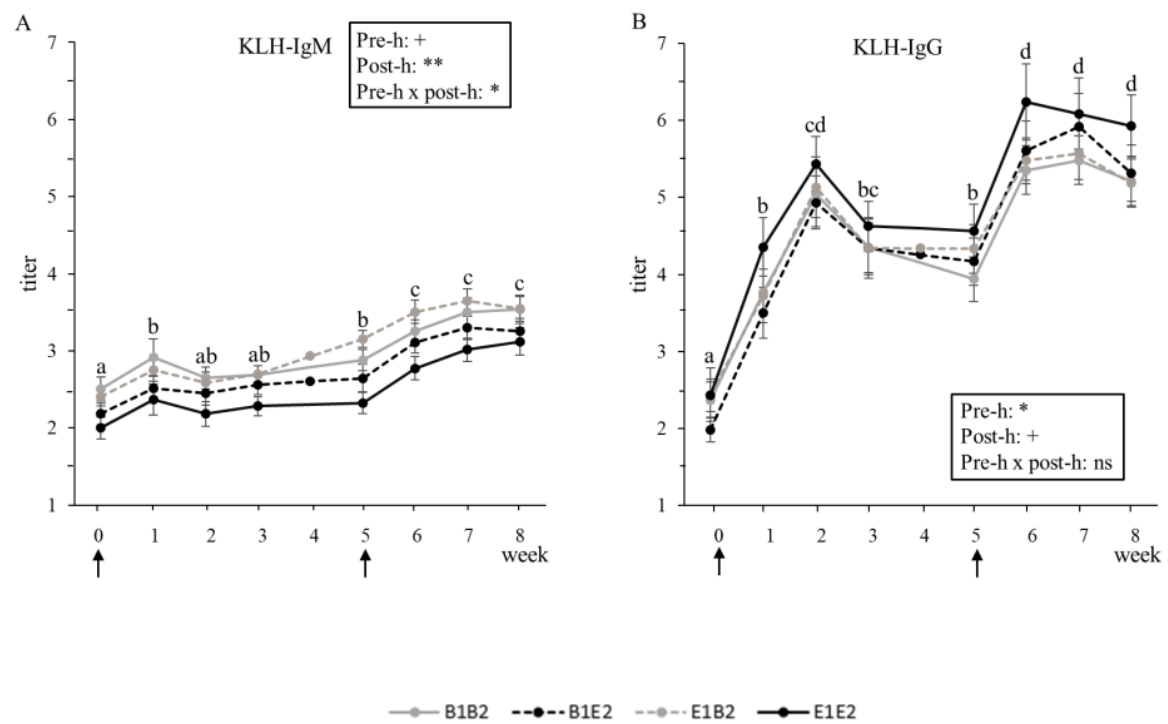

Figure 7.4: Means and SEM of specific KLH-IgM (A) and KLH-IgG (B) antibody titers over 8 weeks in pigs ( $n=62$ ) exposed to different housing situations (B1B2 and E1E2: 
housed in barren respectively enriched pens throughout the experiment; B1BE2 and E1B2: experiencing a change in environment from barren to enriched respectively vice versa from 47 days of age) in response to primary (at day 74 , week=0) and secondary (at day 109, week=5) immunization with KLH-TNP antigen. abcSampling day effects are indicated in the figure: days lacking a common letter differ significantly. Housing effects are presented in the text and in Figure 7.2. Arrows show the immunization moments. Pre-h and post-h indicate early life housing and later life housing, respectively. Significances of differences are indicated: ${ }^{* *} p<0.001,{ }^{*} p<0.05$, $0.05<^{+} p<0.10$, and $n s$ indicates non-significance (exact $p$-values can be found in the text).

Overall antibody response. Figure 7.4A shows changes in specific KLH-IgM responses over time. KLH-IgM titers were affected by sampling day $\left(F_{(7,413)}=30.57, p<0.001\right)$, showing an increase one week after the first and second immunizations. The change in IgM antibody titers over time did not depend on housing (i.e., no housing and sampling day interaction), which indicates there was no difference in time course (kinetics) of the IgM response to KLH.

Figure 7.5A shows the effect of the combination of pre- and post-housing conditions on KLH-IgM titers following primary and secondary immunization. Overall, KLH-IgM titers were affected by post-housing $\left(F_{(1,57)}=49.46, p<0.001\right)$ and tended to be affected by pre-housing $\left(F_{(1,57)}=3.04, p=0.086\right)$. Importantly, the effect of post-housing depended on its combination with the early life conditions of the pigs (pre-housing $\times$ post-housing interaction, $F_{(1,57)}=7.03$, $p=0.010$ ). Post hoc analysis showed that B1B2 and E1B2 pigs had the highest titers, followed by B1E2 and then by E1E2 (Figure 7.5A, $p<0.05$ for all). Thus, the switch from barren to enriched housing lowered antibody titers in B1 pigs 
(B1E2 < B1B2), whereas the switch from enriched to barren housing increased titers in E1 pigs (E1B2 > E1E2). In B2 pigs, there was no difference in antibody titers between pigs originating from early life enriched or barren pens $(E 1 B 2=B 1 B 2)$, whereas in E2 pigs, pigs coming from barren pens had higher titers than those from enriched early-life pens (B1E2 > E1E2, Figure 7.5A).

Primary antibody response. KLH-IgM titers in the 5-week period after first immunization were affected by post-housing (B2: $2.72 \pm 0.05, E 2: 2.35 \pm 0.05$, $\left.F_{(1,57)}=31.20, p<0.001\right)$, and tended to be affected by the pre-housing $\left(F_{(1,57)}=3.30, p=0.074\right)$ and the pre-housing $\times$ post-housing interaction $\left(F_{(1,57)}=2.90, p=0.094\right)$, similar as described for the overall (primary plus secondary) response.

Secondary immunization. Also after the second immunization KLH-IgM titers in the 3-week period were affected by post-housing $\left(F_{(1,56)}=32.39, p<0.001\right)$, and the interaction between pre-housing and post-housing, $F_{(1,56)}=8.60$, $\mathrm{p}=0.005)$. Post hoc analysis showed that E1E2 pigs $(2.81 \pm 0.08)$ had lower titers than E1B2 pigs ( $3.46 \pm 0.08, \mathrm{p}<0.05)$, with levels of B1B2 (3.29 \pm 0.09$)$ and B1E2 (3.08 \pm 0.08$)$ in between.
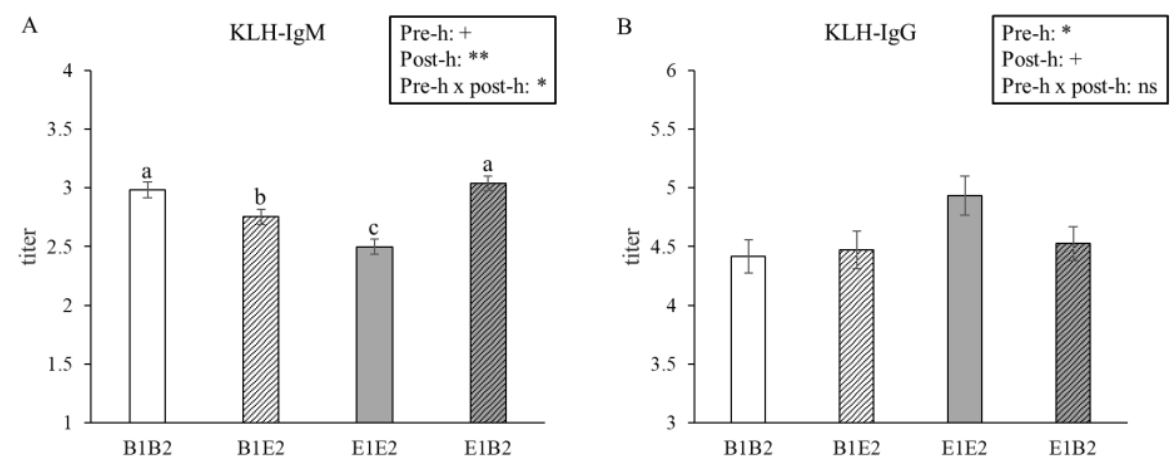
Figure 7.5: Means and SEM of specific KLH-IgM (A) and KLH-IgG (B) titers over 8 weeks in pigs ( $n=62)$ exposed to different housing situations (B1B2 and E1E2: housed in barren respectively enriched pens throughout the experiment; B1BE2 and E1B2: experiencing a change in environment from barren to enriched respectively vice versa from 47 days of age) in response to primary and secondary immunization with $\mathrm{KLH}$ TNP antigen. ${ }^{a b c}$ Groups lacking a common letter significantly differ. Pre-h and post-h indicate early life housing and later life housing, respectively. Significances of differences are indicated: ${ }^{*} p<0.001,{ }^{*} p<0.05,0.05<^{+} p<0.10$, and ns indicates nonsignificance (exact $p$-values can be found in the text).

\subsubsection{KLH-IgG}

Before immunization. Housing did not affect KLH-lgG titers before immunization (pre-housing: $F_{(1.57)}=1.54, p=0.220$, post-housing: $F_{(1,57)}=0.97$, $p=0.329$, pre-housing $\times$ post-housing: $\left.F_{(1,57)}=0.82, p=0.370\right)$.

Overall antibody response. KLH-IgG titers were affected by sampling day $\left(F_{(7,413)}=48.47, p<0.001\right)$, showing an increase both after the first and second immunization (Figure 7.4B). The primary antibody response showed a peak after two weeks, after which titers declined. The secondary immune response peaked after one week, and high titers were retained during the two weeks thereafter (Figure 7.4B). The change in IgG antibody titers over time did not depend on housing (i.e., no housing $\times$ sampling day interactions).

The effect of the combination of pre- and post-housing conditions on overall KLH titers is shown in Figure 5B. KLH-IgG titers were affected by pre-housing $\left(F_{(1,57)}=5.09, p=0.028\right)$, and tended to be affected by post-housing $\left(F_{(1,57)}=3.24\right.$, $p=0.077$ ) in the same direction, which showed that $B$ pigs had or tended to 
have lower titers than E pigs (B1: $4.44 \pm 0.11$ vs. E1: $4.73 \pm 0.11$, B2: $4.47 \pm$ 0.10 vs. E2: $4.70 \pm 0.12$ ).

Primary immunization. In the 5-week period after first immunization KLH-IgG titers tended to be affected by pre-housing $\left(F_{(1,57)}=3.85, p=0.055\right)$, with higher levels for pre-housing E1 pigs $(4.13 \pm 0.12)$ than for B1 pigs ( $3.84 \pm 0.12$ ).

Secondary immunization. KLH-IgG titers in the 3-week period after second immunization were affected by post-housing $\left(F_{(1,56)}=5.05, p=0.029\right)$, as $B 2$ pigs $(5.06 \pm 0.12)$ had lower titers than E2 pigs $(5.47 \pm 0.15)$.

\subsubsection{The effect of housing conditions on peripheral blood leukocyte subpopulations}

Pre- and/or post-housing did not affect relative frequencies of $\mathrm{CD}^{+} \mathrm{T}$ cells, $\mathrm{CD}^{+}$helper T cells, NK cells, and B cells both before and after immunization (for all means and $p$-values, see Supplementary Tables 1 and 2 for day 74 and 130 , respectively).

Before immunization (day 74). At day 74, the percentage of lymphocytes $\left(F_{(1,55)}=8.16, p=0.006\right)$, granulocytes $\left(F_{(1,54)}=3.70, p=0.060\right)$, $D C \quad\left(F_{(1,54)}=4.30\right.$, $p=0.043)$, and the SLA-II expression on monocytes $\left(F_{(1,54)}=6.05, p=0.003\right)$ tended to be affected or were affected by pre-housing. E1 pigs had a higher percentage of lymphocytes (B1: $41.2 \pm 1.6, \mathrm{E} 1: 47.2 \pm 1.5 \%$ ) and DC (B1: 4.7 \pm 0.5 , E1: $6.1 \pm 0.7 \%$ ) compared to B1 pigs, and showed a higher SLA-II expression on monocytes (B1: $524.1 \pm 14.8$, E1: $611.7 \pm 23.9 \mathrm{MFI}$ ), while the percentage of granulocytes tended to be lower for E1 (B1: $34.8 \pm 1.7$, E1: 30.6 $\pm 1.5 \%)$. SLA-II expression on monocytes was also affected by post-housing $\left(F_{(1,54)}=5.75, p=0.020\right)$, and the expression was higher for B2 pigs than $E 2$ pigs 
(B2: $1704.2 \pm 110.4$, E2: $1415.9 \pm 49.7$ ). Pre-housing and post-housing tended to interact in their effect on the percentage of monocytes $\left(F_{(1,54)}=3.34\right.$, $p=0.073$, Figure 7.6C1) and SLA-II expression on $D C\left(F_{(1,54)}=2.83, p=0.098\right.$, Figure 7.6G1). When analysing possible effects of housing conditions on adaptive immune cells, only an effect of post-housing on the percentage of $\mathrm{CD} 4^{+} \mathrm{CD} 8 \alpha^{+} \mathrm{T}$ cells was observed, with higher percentages for $\mathrm{B} 2$ pigs (12.2 \pm $0.9 \%)$ than for $E 2$ pigs $(9.4 \pm 0.5 \%, F(1,55)=9.49, p=0.003)$. No other effects of pre- or post-housing were found on the cells mentioned above, and neither an effect on the granulocyte to lymphocyte ratio was found at day 74 .

After immunization (day 130). At day 130, the percentage of granulocytes $\left(F_{(1,50)}=3.23, p=0.079\right)$, monocytes $\left(F_{(1,50)}=3.67, p=0.061\right)$, and the SLA-II expression on monocytes $\left(F_{(1,50)}=4.49, p=0.039\right)$ tended to be affected or was affected by pre-housing. E1 pigs tended to have a higher percentage of granulocytes (B1: $34.7 \pm 1.6, \mathrm{E} 1: 38.3 \pm 1.7 \%$ ) and had higher SLA-II expression on monocytes (B1: $1691.0 \pm 102.9$, E1: $1942.4 \pm 88.4 \mathrm{MFI})$, but tended to have lower percentage of monocytes (B1: $7.0 \pm 1.1$, E1: $5.4 \pm 0.4 \%$ ) than $B 1$ pigs. The percentage of lymphocytes $\left(F_{(1,55)}=5.19, p=0.027\right)$, granulocytes $\left(F_{(1,50)}=13.49, p<0.001\right)$, and $C D 4{ }^{+} C D 8 \alpha^{+} T$ cells $\left(F_{(1,55)}=4.66\right.$, $p=0.035$ ) were affected by post-housing. E2 pigs had a higher percentage of lymphocytes (B2: $47.5 \pm 1.7$, E2: $50.8 \pm 1.7 \%$ ), but a lower percentage of granulocytes (B2: $39.6 \pm 1.6$, E2: $33.6 \pm 1.6 \%$ ) and $C D 4^{+} C D 8 \alpha^{+} T$ cells (B2: 13.3 \pm 1.0$, E2: $11.57 \pm 0.7 \%)$ than B2 pigs. The ratio of granulocytes to lymphocytes was affected by both pre- $\left(F_{(1,50)}=4.57, p=0.038\right)$ and posthousing $\left(F_{(1,50)}=14.16, p<0.001\right)$, but in different directions (B1: $0.62 \pm 0.04$, E1: $0.74 \pm 0.06, B 2: 0.76 \pm 0.05, E 2: 0.60 \pm 0.05)$. Pre- and post-housing interacted in their effect on the percentage of $D C\left(F_{(1,50)}=4.64, p=0.036\right)$, 
gamma-delta $T$ cells $(F(1,55)=5.60, p=0.022)$, and $C D 8 \alpha^{+}$cytotoxic $T$ cells $(F(1,55)=4.93, p=0.031)$. Post hoc analysis showed that the switch from barren to enriched housing reduced the percentage of DC as compared with enriched housing throughout life (B1E2 < E1E2, $p<0.05$, Figure 7.6E2), whereas B1B2 pigs and E1B2 pigs had levels in between. Conversely, B1E2 pigs had a higher relative frequency of gamma delta T cells than B1B2 pigs $(p<0.05$, Figure $7.6 \mathrm{H} 2)$, with E1E2 and E1B2 in between. The percentage $\mathrm{CD} 8 \alpha^{+}$cytotoxic T cells was also affected by the pre-housing $\times$post-housing interaction, but no significant pairwise differences were found, even though both switched groups (E1B2 and B1E2) seemed to have reduced levels (Figure 7.612). No other effects of pre- or post-housing were found on the cells mentioned above, and neither an effect on the SLA-II expression on DC was found at day 130.

\subsubsection{Coping style effects}

Coping style did not affect $\lg$ titers before immunization $\left(F_{(1,57)}=0.93\right.$, p=0.543), but after immunization, HR pigs had overall lower titers of KLH-IgM $(2.73 \pm 0.05)$ than LR pigs $\left(2.91 \pm 0.05, F_{(1,57)}=11.15, p=0.002\right)$, which held for the primary immune response (HR: $2.43 \pm 0.05, L R: 2.65 \pm 0.05, F_{(1,57)}=11.06$, $p=0.002$ ) and tended to hold for the secondary immune response (HR: 3.10 \pm 0.06 ; LR: $\left.3.23 \pm 0.06, F_{(1,56)}=2.99, p=0.090\right)$. Coping style did not affect IgG responses. Before immunization, coping style affected or tended to affect the percentage of monocytes (HR: $5.4 \pm 0.2$, LR: $4.8 \pm 0.2, F_{(1,54)}=5.30, p=0.025$ ) and the SLA-II expression on monocytes (HR: $1674.4 \pm 111.0$, LR: $1454.1 \pm$ 59.6, $\left.F_{(1,54)}=3.60, p=0.063\right)$. On day 130 , coping style tended to affect NK cell percentages (HR: $7.8 \pm 0.6$, LR: $\left.9.5 \pm 0.8 \%, F_{(1,55)}=3.54, p=0.065\right)$. Coping style 
also affected and tended to affect the percentage of $C D 8 \alpha^{+}$cytotoxic T cells before (HR: $21.7 \pm 0.7$, LR: $\left.24.6 \pm 1.0 \%, F_{(1,55)}=5.25, p=0.026\right)$, and after immunization (HR: $18.7 \pm 0.89$, LR: $21.4 \pm 1.1 \%, F(1,55)=3.83, p=0.055)$.

\subsubsection{Sex effects}

Sex did not affect IgM responses, but sex effects were found on KLH-IgG and some leukocyte subsets. Sex did not affect IgG titers before immunization $\left(F_{(1,57)}=0.64, p=0.432\right)$, while following immunization, females $(4.39 \pm 0.11)$ had overall lower KLH-lgG titers than males $(4.76 \pm 0.10)\left(F_{(1,57)}=8.39\right.$, $p=0.005$ ), which was found for both the primary (F: $3.82 \pm 0.14, M: 4.14 \pm$ $\left.0.12, F_{(1,57)}=4.16, p=0.046\right)$ and secondary immune response ( $F: 5.05 \pm 0.14$, $\left.M: 5.46 \pm 0.13, F_{(1,56)}=6.42, p=0.014\right)$. Sex also affected the percentage of CD4 ${ }^{+} \mathrm{CD} 8 \alpha^{+}$T cells (F: $\left.12.1 \pm 1.0, \mathrm{M}: 9.6 \pm 0.5 \%, \mathrm{~F}_{(1,55)}=6.59, \mathrm{p}=0.013\right)$, and tended to affect NK cells ( $\left.F: 20.8 \pm 2.0, M: 16.8 \pm 1.5 \%, F_{(1,55)}=2.83, p=0.098\right)$ and the SLA-II expression on DC (F: $15462.0 \pm 1438.9$, M: $18514.4 \pm 1217.3$ $\left.\mathrm{MFI}, \mathrm{F}_{(1,54)}=2.92, \mathrm{p}=0.094\right)$ on day 74 . No sex effects were found on day 130 . 
Before immunization (day 74)
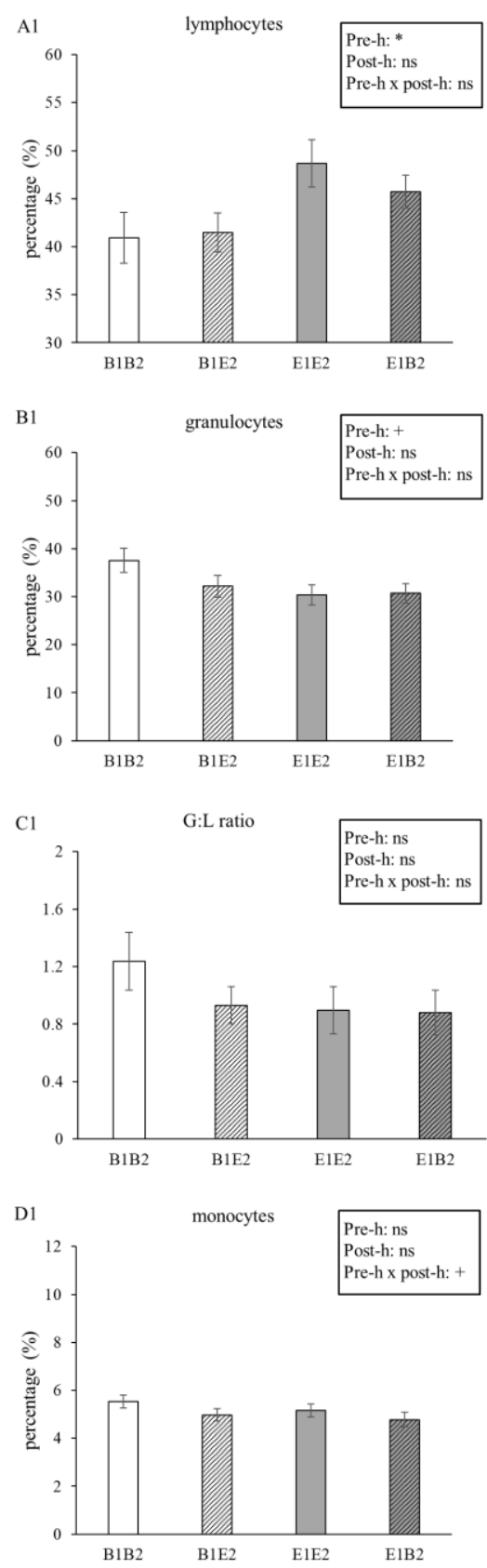

After immunization (day 130)
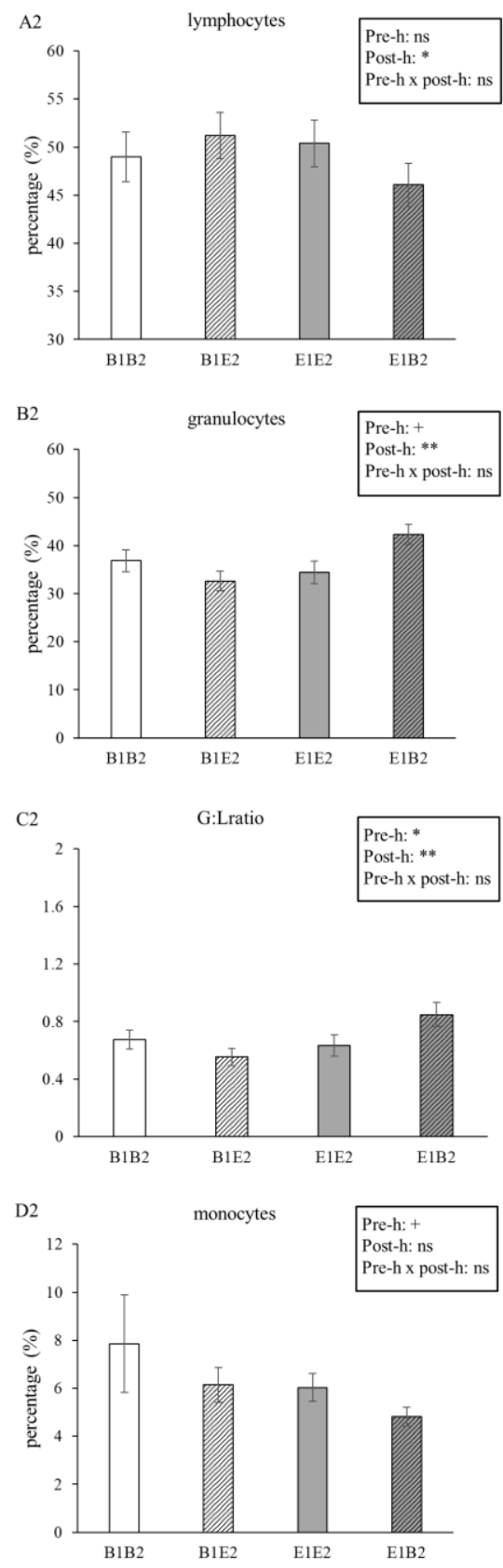

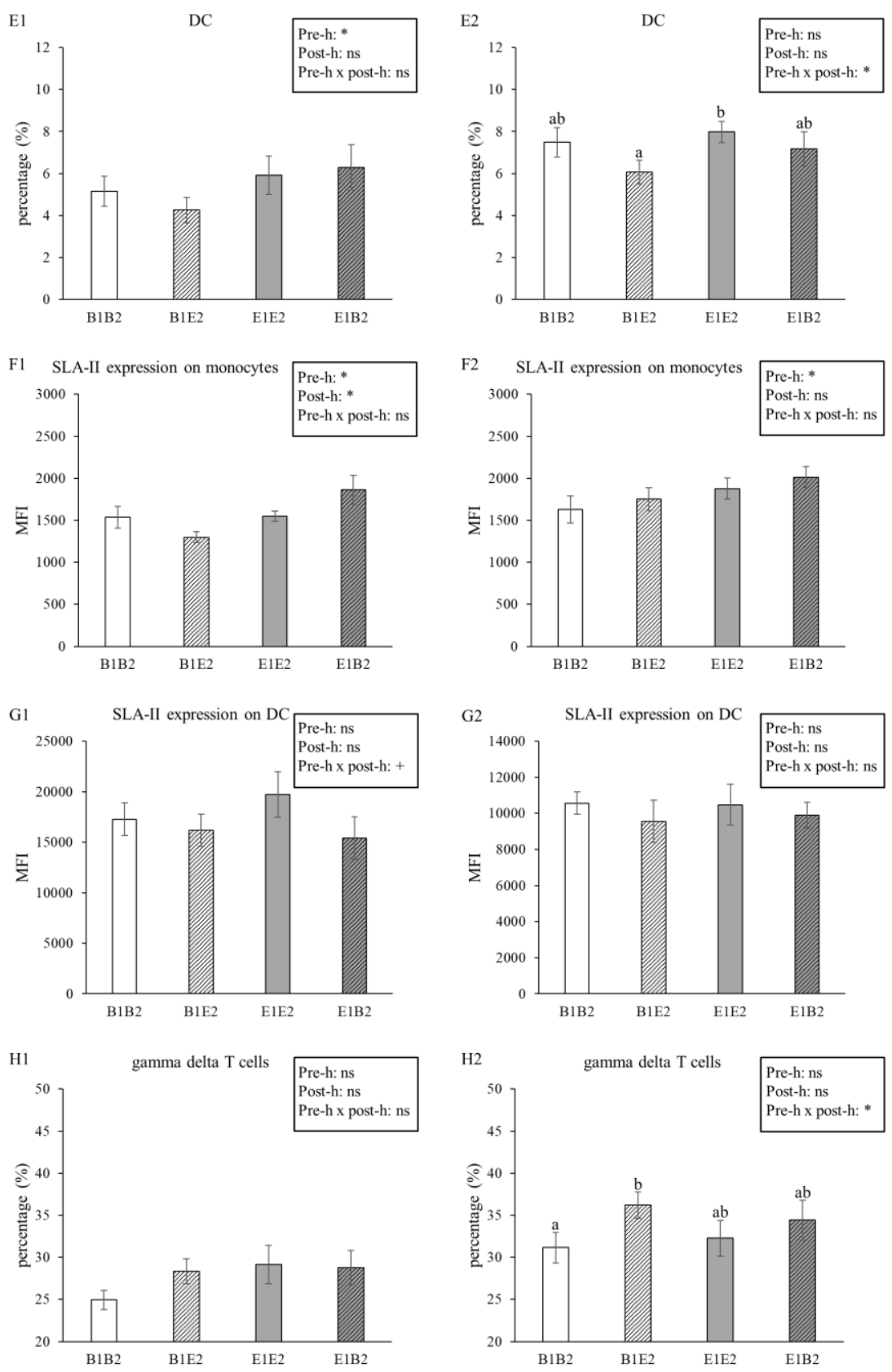

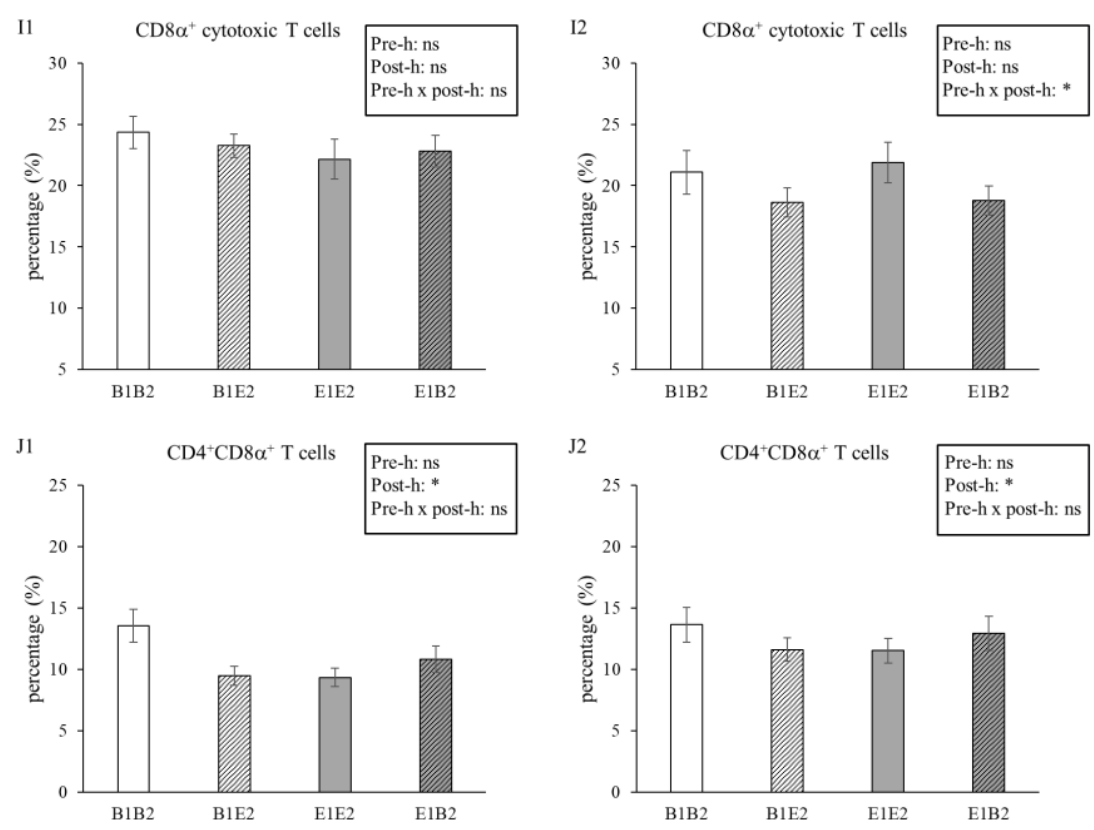

Figure 7.6: Means and SEM of the percentage of lymphocytes, granulocytes, monocytes, dendritic cells (DC), the granulocyte/lymphocyte (G:L) ratio, the SLA-II expression on monocytes, $D C$, the percentage of gamma delta $T$ cells, $C D 4^{+} C D 8 \alpha^{+} T$ cells, and $C D 8 \alpha^{+}$cytotoxic $T$ cells before immunization, on day 74 (on the left side), and after immunization, on day 130 (on the right side), in pigs ( $n=62$ ) exposed to four different housing conditions (B1B2 and E1E2: housed in barren respectively enriched pens throughout the experiment; B1BE2 and E1B2: experiencing a change in environment from barren to enriched respectively vice versa from 47 days of age) through primary and secondary immunization with KLH-TNP antigen on day 74 and 109. Significant differences between four housing groups are indicated by letter $a$ and $b$ ( $p<0.05)$. Pre-h and post-h indicate early life and later life housing, respectively. Significances of differences are indicated: ${ }^{* *} p<0.001,{ }^{*} p<0.05,0.05<{ }^{+} p<0.10$, and ns indicates non-significance. 


\subsection{Discussion}

In this study, we investigated the effects of early and later life environmental enrichment in pigs on specific IgM and IgG antibody responses to the T-celldependent antigen KLH-TNP as a parameter of specific immunity and memory. In addition, the effects of early life and later life housing experience on the frequencies of cells in the blood related to innate and adaptive immunity were measured. We chose to use the model antigen KLH-TNP to induce mild primary and secondary immune responses and refrained from using adjuvant as this may have overruled potential housing effects. The IgG and low IgM responses likely reflected T-cell-dependent antibody responses (Butler et al. 2002). We found long-lasting effects of both housing before the switch of environment (early life environment) and later life housing on several immune parameters, and, moreover, the effects of early and later life conditions often interacted, thus a clear effect of the switch was found. This study reveals that housing conditions and early life housing history can affect specific antibody responses and the frequencies of immune cells, reflecting immune status, in the blood, which suggests differences in immune competence. Both coping style and sex affected some of the immune parameters and will be discussed separately.

\subsubsection{Early life housing}

We found that early life conditions (from birth until 47 days of age) exerted an effect on humoral immune responses (KLH-IgG) and immune cells (mostly on innate cells) in later life, for some up to 130 days. For distribution of several leukocyte subsets and KLH-IgM titers, however, the impact of early life housing depended on its combination with current housing, as shown by 
interaction effects (see below for a discussion on those). Pigs from barren housing conditions in early life showed an overall lower IgG response to KLH. Separate analysis of the primary and secondary immune responses revealed that early life housing mainly affected the former, whereas the early life effect seemed to be overruled by the current housing effect on the secondary IgG response to KLH. The (early life) housing effects on IgG titers are in contrast with other studies in which no effects of enrichment throughout life, either with straw and extra space (de Groot et al. 2000) or with sawdust and extra outdoor space (Merlot et al. 2012) on IgG responses to KLH were found (de Groot et al. 2000). The discrepancy between our and these other studies might be caused by a difference in age at (primary) immunization, in timing of sampling as related to immunization and/or in substrates used as enrichment (in our study, apart from straw bedding or sawdust also peat and extra toys).

Early life enrichment, as compared with barren housing before the switch, not only increased the IgG response to $\mathrm{KLH}$. It also increased percentages of lymphocytes (day 74), DC (day 74), granulocytes (day 130), the SLA-II expression on monocytes (day 74 and 130), while it tended to decrease the percentage of granulocytes at day 74 and the percentage of monocytes at day 130, revealing a (mild) effect of early life enrichment on the subsets of immune cells in peripheral blood.

It is known that environmental enrichment can positively influence behaviour and welfare of pigs, but how early life environmental enrichment exactly influences the immune system is not clear yet. Different effects of enrichment may have played a role here. Firstly, the early oral sampling of 
rooting substrates and thus enlarged antigenic exposure facilitated by environmental enrichment may have had an impact on intestinal maturation and early microbial colonization, and, consequently, on regulation of innate and adaptive immunity (Mulder et al. 2009; Schokker et al. 2014). It has been shown that environmental factors during the early postnatal period that affect microbial exposure, such as natural vs. isolator-rearing (Mulder et al. 2009), outdoor vs. indoor rearing (Mulder et al. 2009), diet (Buddington and Sangild 2011; Mackie et al. 1999) or neonatal antibiotic treatment (Schokker et al. 2014), can influence the establishment of gut microbiota in pigs. The gut microbiota play a role in the communication between the gut and brain as well, which is established via a range of chemical, hormonal, immunological and neural signals (reviewed in Kraimi et al. 2019). Evidence is accumulating that this 'gut-brain axis' can influence stress responses, behaviour and immunity in animals (reviewed in Kraimi et al. 2019; Mayer et al. 2015).

Secondly, environmental enrichment can influence the stress level and mental state of pigs as it partly satisfies their behavioural need to explore (de Jong et al. 2000; Douglas et al. 2012). It could therefore influence their immune status and responses (Koh 1998; Matsunaga et al. 2013), as the brain and immune system form a bidirectional communication network, amongst others via the hypothalamic-pituitary-adrenal (HPA) axis (review in Maier and Watkins 1998). Several studies point to a difference in HPA axis functioning between pigs from barren and enriched housing (Beattie et al. 2000; de Jong et al. 2000; Reimert et al. 2014a), some of which seem to be established in early life. For instance, absence of enrichment materials up till 4 weeks of age, led to a blunted circadian cortisol rhythm, possibly reflecting 
chronic stress, in later life, at 21 weeks of age (Munsterhjelm et al. 2010). In addition, we found that pigs housed in the barren environment in early life were more sensitive to reward loss (Luo et al. 2019b), supporting a long-term effect on their adaptive capacity and mental state. It has been found that psychosocial factors can affect functioning of the immune system (Barak 2006; Maier and Watkins 1998). For instance, in humans, adverse experiences in early life are associated with long-term effects on both the innate and adaptive immune system, even though it does not appear to affect all elements of the immune system to the same extent (review in Elwenspoek et al. 2017). In pigs, 'cognitive enrichment', allowing pigs to successfully cope with a demanding task rewarded by small portions of their food (Puppe et al. 2007), has been reported to result in an increased IgG concentration and in vitro T-cell proliferation to ConA, reduced LPS-induced proliferation of B cells and accelerated wound healing (Ernst et al. 2006). Given that the cognitive enrichment used did not alter antigenic exposure, this suggests that, also in pigs, one way in which enrichment influences their immune system could be via its effect on their mental state.

\subsubsection{Actual housing conditions and their interaction with early life enrichment}

Our study revealed an impact of the actual housing conditions on both innate and specific immunity of the pigs as well. KLH-IgM titers before immunization were affected by actual housing, with higher titers for B2 pigs than for E2 pigs. These anti-KLH antibodies circulating in absence of antigenic stimulation are also referred to as natural antibodies (Casali and Notkins 1989). Natural antibodies are important as first line of defence against microbial infection 
(Coutinho et al. 1995), and participate in physiological activities (Panda and Ding 2015). Also in other studies barren and enriched housed pigs were found to differ in natural (auto)antibody levels binding several antigens including KLH (Luo et al. 2017a; Luo et al. 2017b; Reimert et al. 2014b), although differences were not always in the same direction as in our study. Our results on particularly IgM binding KLH are in contrast with other studies, which showed higher levels of natural KLH-IgM antibodies in enriched housed pigs (Reimert et al. 2014b), or no housing effect on KLH-IgM antibodies (Luo et al. 2017a). The reasons could be a difference in the age at which the enrichment started (before or after weaning), and/or in the exact type of enrichment applied. Other studies used enrichment with straw only, whereas we also provided peat and sawdust to the pigs, as well as extra space. Also, the barren conditions applied vary between studies, as we still provided two simple toys that are similar to those used on many commercial pig farms.

Actual housing conditions also affected the frequencies of T cells before and after immunization, as B2 pigs had a higher percentage of $\mathrm{CD} 4^{+} \mathrm{CD} 8 \alpha^{+} \mathrm{T}$ cells than E2 pigs. In another study, however, enriched and barren housed pigs did not differ in the percentage of $C D 4^{+} C D 8 \alpha^{+}$T cells (de Groot et al. 2000), possibly, as indicated above, due to differences in experimental design, sample size (larger in our study) and type of enrichment. On day 130, E2 pigs had a higher percentage of lymphocytes than B2 pigs, which is in line with the early life enrichment effect on lymphocyte percentage at day 74 . We speculate that after immunization, similar to the KLH-IgG response, the effect of current housing on percentage of lymphocytes overruled the effect of early life at day 130 , as there was no early life effect anymore. Actual housing 
also affected SLA-II expression on monocytes on day 74, with higher levels for barren-housed pigs.

Pigs in barren actual housing also had higher percentages of granulocytes than those in enriched pens on day 130. The granulocyte/lymphocyte ratio on this was affected by both early life and current housing conditions, but in different directions. Adding up the early life and current housing effects revealed the highest ratio in pigs that switched from enriched to barren conditions, and, conversely, the lowest ratio in pigs exposed to the opposite switch. The granulocyte to lymphocyte ratio is a stress marker, and increases for instance following surgical stress in humans (Tabuchi et al. 2011), restraint stress in rodents (MORODA et al. 1997) and transport stress in pigs (Sutherland et al. 2012). Merlot et al.(Merlot et al. 2012) reported higher granulocyte to lymphocyte ratios in Large White pigs kept in conventional, rather barren, pens, as compared to those provided with sawdust bedding and an outdoor area, suggesting also a long-term stress effect on this marker. The combined impacts of early life and current housing on the granulocyte to lymphocyte ratio in the current study might thus point to increased stress levels in pigs that switched from enriched to barren housing.

Notably, the current study revealed that early life and later life housing often interacted in their effects on both specific and innate immunity variables. Thus, the effects of actual housing often depended on the early life history of the pigs, demonstrating an impact of the switch in housing conditions. Both the switch from barren to enriched and from enriched to barren housing changed KLH-IgM titers. In non-switched animals, IgM titers were higher for B1B2 than for E1E2, and switched animals moved towards the 
titers of the non-switched animals kept in their later life environment from birth onwards. Still, however, pigs in enriched conditions throughout life (E1E2) differed from enriched-housed pigs originating from early life barren housing (B1E2) in KLH-IgM titers, illustrating that the later life conditions did not completely overrule the effects of housing before the switch. The switch in housing also affected the percentage of DC, CD8 $\alpha^{+}$cytotoxic T cells (but no pairwise difference here) and gamma delta $T$ cells at day 130 . The switch effect on the percentage of DC and gamma delta T cells, however, was only found in the pigs that changed from barren to enriched housing conditions, but not in the pigs that switched from enriched to barren housing. Notably, pigs kept in stable enriched vs. barren conditions from birth onwards did not differ in these leukocyte subpopulations mentioned before, but it was the switch that resulted in a change. Previous studies have shown that pigs that switched from enriched to barren housing conditions show as much behavioural signs of stress (Bolhuis et al. 2006) and pessimistic mood (Douglas et al. 2012) as barren non-switched pigs or even more (Munsterhjelm et al. 2009) (Luo et al., in preparation), suggesting that loss of enrichment may be more detrimental than barren housing throughout life (Latham and Mason 2010). Less is known about the impact of switching from barren to enriched housing, although some studies demonstrated that pigs originating from barren pens showed more enrichment-directed behaviour than pigs reared in enriched pens (Bøe 1993; Kelly et al. 2000) and a lower occurrence of gastric lesions (Bolhuis et al. 2006). Studies in other species found that switching from group housing to individual housing or vice versa had an impact on some indicators of immune function (NK cells) (HoffmanGoetz et al. 1991). 
To our knowledge, this is the first study to show that both early life and later life enrichment, and, notably a switch in housing conditions can influence specific antibodies (as a parameter of immune responsiveness) and leukocyte subpopulations in pigs. The underlying mechanism is not known yet. Both the difference in exposure to antigens in the current housing conditions related to the absence or presence of rooting material (Lewis et al. 2013; Mulder et al. 2009), and the changes in behaviour (Bolhuis et al. 2005; Puppe et al. 2007), stress level (Bolhuis et al. 2006; de Jong et al. 2000; Marashi et al. 2003) and mental state (Asher et al. 2016; Douglas et al. 2012; Luo et al. 2019a) may have influenced immune response, memory and status (reviewed in Farrell et al. 2018; Lopes 2017; Marketon and Glaser 2008). Based on the latter, i.e. a potential influence of stress and mental state, one might expect the switch from barren to enriched housing to give opposite results on immune function compared with the switch from an enriched to a barren environment, which did not hold for all immune parameters in this study, so other influences of enrichment likely played a role as well. Either switch induced alterations in specific $\lg M$ antibody responses and the switch from barren to enriched changed levels of some of immune cells, suggesting that the immune system not only responds to early life and actual housing conditions, but also to a change in environment. Notably, this was not due to relocation to a new pen per se as also the pigs remaining in barren or enriched pens throughout life were relocated to new pens at the time of the switch. This impact of early life conditions and of a change in housing conditions means that these need to be taken into account when assessing immune competence in pigs. Further studies are needed to investigate how changes in housing affect innate and adaptive immunity, and other 
components of the immune system that were not included in the present study, and to study their potential impact on disease resistance, vaccine responses and immune competence. A recent study provided evidence that enriched housed pigs more effectively battle an infection with porcine reproductive and respiratory syndrome virus (PRRSV) and Actinobacillus pleuropneumoniae, as demonstrated by a faster viral clearance and less lung damage, but did not address the impact of early life conditions (van Dixhoorn et al. 2016).

\subsubsection{Coping style}

Although individual characteristics of pigs were not the main topic of our study, we balanced treatments for coping style, by splitting our population of pigs into low-resisters (LR) and high-resisters (HR) based on their backtest response, without selecting extremes, simply to account for potential effects of this personality trait on the immune parameters measured. The response of pigs in the backtest has been shown to relate to a range of behavioural and (neuro)physiological variables (e.g. Bolhuis et al. 2000; Kanitz et al. 2019; O'Malley et al. 2019) and may therefore indicate a pig's coping style. LR pigs had higher specific KLH-IgM antibody titers than HR pigs in this study, which is alike the previous studies demonstrating a higher specific humoral immune response in LR pigs (Hessing et al. 1995; Schrama et al. 1997), although others found no difference in adult pigs (Geverink et al. 2004) or found the effect of coping style to be housing-dependent (Bolhuis et al. 2003). Previously, also other immune differences were reported, suggesting LR pigs to have a lower cellular immunity (Bolhuis et al. 2003; Hessing et al. 1995), a lower complement activity via the alternative pathway (Reimert et al. 2014b) and 
a different gene expression signature for processes acting on cell communication, vasculogenesis and blood coagulation in blood mononuclear cells (Otten et al. 2007). It should be noted that in most of these studies the LR and HR selected were more at the extremes of the population. In this study, coping style did not affect leukocyte distribution to a large extent, as only subtle differences were found. HR pigs had a lower percentage of CD8 $\alpha^{+}$ cytotoxic T cells than LR pigs, and higher percentage of monocytes and the SLA-II expression on monocytes before immunization. This further confirms immune differences between the two types of pig, which may be related to their differences in several (neuro)physiological systems (Bolhuis et al. 2000; Hessing et al. 1994; Ruis et al. 2001) that communicate with the immune system (Koolhaas 2008).

\subsubsection{Sex effects}

Differences between females and males were found for a number of immune parameters, in line with other studies reporting some influence of sex on immunity (Luo et al. 2017b; Marin et al. 2006; Reimert et al. 2014b). This could be due to the fundamental hormonal and genetic differences between females and males, and also relate to how females and males deal with stress (Darnall and Suarez 2009; Oertelt-Prigione 2012; Reimert et al. 2014a; Stefanski and Grüner 2006).

\subsection{Conclusions}

In conclusion, both early life and later life enrichment, and, notably a switch in housing conditions influenced specific antibodies and leukocyte 
subpopulations in pigs. The current study implies that the early life history of animals and the (mis)match with their current environment could thus be of major importance for functioning of their immune system, which may have consequences for their responses to vaccination and disease resistance. Further research is needed to investigate potential consequences for the pigs' health.

\subsection{Competing interests}

The authors declare no competing interests.

\subsection{Acknowledgements}

The authors thank M. van Marwijk, B. Laurenssen, D.A. van Haarlem and A. Hoek for skilful assistance in conducting the experiment. We are also grateful to the animal caretakers and students involved. 


\subsection{References}

Asher L, Friel M, Griffin K, Collins LM (2016) Mood and personality interact to determine cognitive biases in pigs Biology letters 12:20160402

Bacou E et al. (2017) Acute social stress-induced immunomodulation in pigs high and low responders to ACTH Physiology \& behavior 169:1-8

Barak Y (2006) The immune system and happiness Autoimmunity reviews 5:523-527

Beattie V, O'Connell N, Kilpatrick D, Moss B (2000) Influence of environmental enrichment on welfare-related behavioural and physiological parameters in growing pigs Animal Science 70:443-450

Bøe K (1993) The effect of age at weaning and post-weaning environment on the behaviour of pigs Acta Agriculturae Scandinavica A-Animal Sciences 43:173-180

Bolhuis J, Schouten W, De Jong I, Schrama J, Cools A, Wiegant V (2000) Responses to apomorphine of pigs with different coping characteristics Psychopharmacology 152:24-30

Bolhuis JE, Oostindjer M, Hoeks CW, de Haas EN, Bartels AC, Ooms M, Kemp B (2013) Working and reference memory of pigs (Sus scrofa domesticus) in a holeboard spatial discrimination task: the influence of environmental enrichment Animal cognition 16:845-850

Bolhuis JE, Parmentier HK, Schouten WG, Schrama JW, Wiegant VM (2003) Effects of housing and individual coping characteristics on immune responses of pigs Physiology \& behavior 79:289-296

Bolhuis JE, Schouten WG, Schrama JW, Wiegant VM (2005) Behavioural development of pigs with different coping characteristics in barren and substrate-enriched housing conditions Applied Animal Behaviour Science 93:213-228

Bolhuis JE, Schouten WG, Schrama JW, Wiegant VM (2006) Effects of rearing and housing environment on behaviour and performance of pigs with different coping characteristics Applied Animal Behaviour Science 101:68-85

Buddington R, Sangild PT (2011) Companion animals symposium: development of the mammalian gastrointestinal tract, the resident microbiota, and the role of diet in early life Journal of animal science 89:1506-1519

Butler JE, Weber P, Sinkora M, Baker D, Schoenherr A, Mayer B, Francis D (2002) Antibody repertoire development in fetal and neonatal piglets. VIII. Colonization is required for newborn piglets to make 
serum antibodies to T-dependent and type $2 \mathrm{~T}$-independent antigens The Journal of Immunology 169:6822-6830

Casali P, Notkins AL (1989) Probing the human B-cell repertoire with EBV: polyreactive antibodies and CD5+ B lymphocytes Annual review of immunology 7:513-535

Coutinho A, Kazatchkine MD, Avrameas S (1995) Natural autoantibodies Current opinion in immunology 7:812-818

Cui Y, Hao Y, Li J, Bao W, Li G, Gao Y, Gu X (2016) Chronic heat stress induces immune response, oxidative stress response, and apoptosis of finishing pig liver: a proteomic approach International journal of molecular sciences 17:393

Darnall BD, Suarez EC (2009) Sex and gender in psychoneuroimmunology research: past, present and future Brain, Behavior, and Immunity 23:595-604

de Groot J, de Jong IC, Prelle IT, Koolhaas JM (2000) Immunity in barren and enriched housed pigs differing in baseline cortisol concentration Physiology \& behavior 71:217-223

de Jong IC, Prelle IT, van de Burgwal JA, Lambooij E, Korte SM, Blokhuis HJ, Koolhaas JM (2000) Effects of environmental enrichment on behavioral responses to novelty, learning, and memory, and the circadian rhythm in cortisol in growing pigs Physiology \& behavior 68:571-578

De Jonge FH, Bokkers $\mathrm{E}$, Schouten $\mathrm{W}$, Helmond $\mathrm{F}$ (1996) Rearing piglets in a poor environment: developmental aspects of social stress in pigs Physiology \& Behavior 60:389-396

Dhabhar FS (2014) Effects of stress on immune function: the good, the bad, and the beautiful Immunologic research 58:193-210

Douglas C, Bateson M, Walsh C, Bédué A, Edwards SA (2012) Environmental enrichment induces optimistic cognitive biases in pigs Applied Animal Behaviour Science 139:65-73

Elwenspoek MM et al. (2017) Proinflammatory T cell status associated with early life adversity The Journal of Immunology 199:4046-4055

Ernst K, Tuchscherer M, Kanitz E, Puppe B, Manteuffel G (2006) Effects of attention and rewarded activity on immune parameters and wound healing in pigs Physiology \& behavior 89:448-456

Fagundes CP, Glaser R, Kiecolt-Glaser JK (2013) Stressful early life experiences and immune dysregulation across the lifespan Brain, behavior, and immunity $27: 8-12$ 
Farrell AK, Imami L, Stanton SC, Slatcher RB (2018) Affective processes as mediators of links between close relationships and physical health Social and Personality Psychology Compass 12:e12408

Geverink N, Parmentier H, de Vries Reilingh G, Schouten W, Gort G, Wiegant $V(2004)$ Effect of response to backtest and housing condition on cellmediated and humoral immunity in adult pigs Physiology \& behavior 80:541-546

Grimberg-Henrici CG, Vermaak P, Bolhuis JE, Nordquist RE, van der Staay FJ (2016) Effects of environmental enrichment on cognitive performance of pigs in a spatial holeboard discrimination task Animal cognition 19:271-283

Hessing M, Coenen G, Vaiman M, Renard C (1995) Individual differences in cell-mediated and humoral immunity in pigs Veterinary Immunology and Immunopathology 45:97-113

Hessing MJ, Hagels $\varnothing$ AM, Schouten WG, Wiepkema PR, Van Beek JA (1994) Individual behavioral and physiological strategies in pigs Physiology \& Behavior 55:39-46

Hoffman-Goetz L, Simpson JR, Arumugam Y (1991) Impact of changes in housing condition on mouse natural killer cell activity Physiology \& behavior 49:657-660

Kanitz E, Tuchscherer M, Otten W, Tuchscherer A, Zebunke M, Puppe B (2019) Coping style of pigs is associated with different behavioral, neurobiological and immune responses to stressful challenges Frontiers in behavioral neuroscience 13

Kelly H, Bruce J, English P, Fowler V, Edwards S (2000) Behaviour of 3-week weaned pigs in Straw-Flow ${ }^{\circledR}$, deep straw and flatdeck housing systems Applied Animal Behaviour Science 68:269-280

Koh KB (1998) Emotion and immunity Journal of Psychosomatic Research 45:107-115

Koolhaas J (2008) Coping style and immunity in animals: making sense of individual variation Brain, behavior, and immunity 22:662-667

Kraimi $\mathrm{N}$ et al. (2019) Influence of the microbiota-gut-brain axis on behavior and welfare in farm animals: A review Physiology \& behavior:112658

Latham N, Mason G (2010) Frustration and perseveration in stereotypic captive animals: is a taste of enrichment worse than none at all? Behavioural Brain Research 211:96-104

Lewis MC et al. (2012) Direct experimental evidence that early-life farm environment influences regulation of immune responses Pediatric Allergy and Immunology 23:265-269 
Lewis MC, Patel DV, Fowler J, Duncker S, Zuercher AW, Mercenier A, Bailey M (2013) Dietary supplementation with Bifidobacterium lactis NCC2818 from weaning reduces local immunoglobulin production in lymphoid-associated tissues but increases systemic antibodies in healthy neonates British Journal of Nutrition 110:1243-1252

Lopes PC (2017) Why are behavioral and immune traits linked? Hormones and behavior 88:52-59

Luo L, Dixhoorn IDE, Reimert I, Kemp B, Bolhuis JE, Parmentier HK (2017a) Effect of enriched housing on levels of natural (auto-) antibodies in pigs co-infected with porcine reproductive and respiratory syndrome virus (PRRSV) and Actinobacillus pleuropneumoniae Veterinary research 48:75

Luo L, Geers R, Reimert I, Kemp B, Parmentier H, Bolhuis J (2017b) Effects of environmental enrichment and regrouping on natural autoantibodies-binding danger and neural antigens in healthy pigs with different individual characteristics animal:1-8

Luo L, Reimert I, de Haas EN, Kemp B, Bolhuis JE (2019a) Effects of early and later life environmental enrichment and personality on attention bias in pigs (Sus scrofa domesticus) Anim Cogn:1-14

Luo L, Reimert I, Graat E, Smeets S, Kemp B, Bolhuis J (2019b) Effects of early life and current housing on sensitivity to reward loss in a successive negative contrast test in pigs Animal cognition:1-10

Mackie RI, Sghir A, Gaskins HR (1999) Developmental microbial ecology of the neonatal gastrointestinal tract The American journal of clinical nutrition 69:1035s-1045s

Maier SF, Watkins LR (1998) Cytokines for psychologists: implications of bidirectional immune-to-brain communication for understanding behavior, mood, and cognition Psychological review 105:83

Marashi V, Barnekow A, Ossendorf E, Sachser N (2003) Effects of different forms of environmental enrichment on behavioral, endocrinological, and immunological parameters in male mice Hormones and Behavior 43:281-292

Marin DE, Taranu I, Pascale F, Lionide A, Burlacu R, Bailly J-D, Oswald IP (2006) Sex-related differences in the immune response of weanling piglets exposed to low doses of fumonisin extract British journal of nutrition 95:1185-1192

Marketon JIW, Glaser R (2008) Stress hormones and immune function Cellular immunology 252:16-26 
Matsunaga $M$ et al. (2013) Brain-immune interaction accompanying odorevoked autobiographic memory PLoS One 8:e72523

Mayer EA, Tillisch K, Gupta A (2015) Gut/brain axis and the microbiota The Journal of clinical investigation 125:926-938

McCullough KC, Schaffner R, Natale V, Kim YB, Summerfield A (1997) Phenotype of porcine monocytic cells: modulation of surface molecule expression upon monocyte differentiation into macrophages Veterinary immunology and immunopathology 58:265-275

Melotti L, Oostindjer M, Bolhuis JE, Held S, Mendl M (2011) Coping personality type and environmental enrichment affect aggression at weaning in pigs Applied Animal Behaviour Science 133:144-153

Merlot E et al. (2012) Health and immune traits of Basque and Large White pigs housed in a conventional or enriched environment Animal 6:1290-1299

MORODA T et al. (1997) Association of granulocytes with ulcer formation in the stomach of rodents exposed to restraint stress Biomedical Research 18:423-437

Mulder IE et al. (2009) Environmentally-acquired bacteria influence microbial diversity and natural innate immune responses at gut surfaces BMC biology $7: 1$

Munsterhjelm C, Peltoniemi OA, Heinonen M, Hälli O, Karhapää M, Valros A (2009) Experience of moderate bedding affects behaviour of growing pigs Applied Animal Behaviour Science 118:42-53

Munsterhjelm C, Valros A, Heinonen M, Hälli O, Siljander-Rasi H, Peltoniemi $O$ (2010) Environmental enrichment in early life affects cortisol patterns in growing pigs Animal 4:242-249

Newberry RC (1995) Environmental enrichment: increasing the biological relevance of captive environments Appl Anim Behav Sci 44:229-243

Nguyen DN, Jiang P, Frøkiær H, Heegaard PM, Thymann T, Sangild PT (2016) Delayed development of systemic immunity in preterm pigs as a model for preterm infants Scientific reports 6:36816

O'Mahony SM et al. (2009) Early life stress alters behavior, immunity, and microbiota in rats: implications for irritable bowel syndrome and psychiatric illnesses Biological psychiatry 65:263-267

O'Malley Cl, Turner SP, D'Eath RB, Steibel JP, Bates RO, Ernst CW, Siegford $J M$ (2019) Animal personality in the management and welfare of pigs Appl Anim Behav Sci 
Oertelt-Prigione S (2012) The influence of sex and gender on the immune response Autoimmunity reviews 11:A479-A485

Oster M, Scheel M, Muráni E, Ponsuksili S, Zebunke M, Puppe B, Wimmers K (2015) The fight-or-flight response is associated with PBMC expression profiles related to immune defence and recovery in swine PLoS One 10:e0120153

Otten W, Kanitz E, Tuchscherer M, Puppe B, Nürnberg G (2007) Repeated administrations of adrenocorticotropic hormone during gestation in gilts: effects on growth, behaviour and immune responses of their piglets Livestock Science 106:261-270

Panda S, Ding JL (2015) Natural antibodies bridge innate and adaptive immunity The journal of immunology 194:13-20

Puppe B, Ernst K, Schön PC, Manteuffel G (2007) Cognitive enrichment affects behavioural reactivity in domestic pigs Applied Animal Behaviour Science 105:75-86

Reimert I, Rodenburg TB, Ursinus WW, Kemp B, Bolhuis JE (2014a) Responses to novel situations of female and castrated male pigs with divergent social breeding values and different backtest classifications in barren and straw-enriched housing Applied animal behaviour science 151:24-35

Reimert I, Rodenburg TB, Ursinus WW, Kemp B, Bolhuis JE (2014b) Selection based on indirect genetic effects for growth, environmental enrichment and coping style affect the immune status of pigs PloS one 9:e108700

Ruis MA, te Brake JH, Engel B, Buist WG, Blokhuis HJ, Koolhaas JM (2001) Adaptation to social isolation: acute and long-term stress responses of growing gilts with different coping characteristics Physiology \& behavior 73:541-551

Schokker D, Zhang J, Vastenhouw SA, Heilig HG, Smidt H, Rebel JM, Smits MA (2015) Long-lasting effects of early-life antibiotic treatment and routine animal handling on gut microbiota composition and immune system in pigs PLoS One 10:e0116523

Schokker D et al. (2014) Early-life environmental variation affects intestinal microbiota and immune development in new-born piglets PLoS One 9:e100040

Schrama J, Schouten J, Swinkels J, Gentry J, de Vries Reilingh G, Parmentier H (1997) Effect of hemoglobin status on humoral immune response of weanling pigs differing in coping styles Journal of animal science 75:2588-2596 
Stefanski V, Grüner S (2006) Gender difference in basal and stress levels of peripheral blood leukocytes in laboratory rats Brain, behavior, and immunity 20:369-377

Summerfield A, Guzylack-Piriou L, Schaub A, Carrasco CP, Tâche V, Charley B, McCullough KC (2003) Porcine peripheral blood dendritic cells and natural interferon-producing cells Immunology 110:440-449

Sutherland M, Bryer P, Davis B, Smith J, McGlone J (2012) The combined effects of transport and food and water deprivation on the physiology of breeding age gilts Livestock science 144:124-131

Tabuchi T, Shimazaki J, Satani T, Nakachi T, Watanabe Y, Tabuchi T (2011) The perioperative granulocyte/lymphocyte ratio is a clinically relevant marker of surgical stress in patients with colorectal cancer Cytokine 53:243-248

van Dixhoorn ID et al. (2016) Enriched housing reduces disease susceptibility to co-infection with porcine reproductive and respiratory virus (PRRSV) and Actinobacillus pleuropneumoniae (A. pleuropneumoniae) in young pigs PloS one 11:e0161832 


\section{Supplementary Information}

Table 1: Means and SEM of the relative frequency of leukocyte subpopulations in pigs exposed to four different housing conditions (B1B2 and E1E2: housed in barren respectively enriched pens throughout the experiment; B1E2 and E1B2: experiencing a change in environment from barren to enriched respectively vice versa from 47 days of age) on day 74 of age, before immunization.

\begin{tabular}{|c|c|c|c|c|c|c|c|}
\hline & B1B2 & B1E2 & E1B2 & E1E2 & Pre-h & Post-h & $\begin{array}{l}\text { Pre-h } x \\
\text { Post-h }\end{array}$ \\
\hline Lymphocytes (\%) & $40.9 \pm 2.7$ & $41.5 \pm 2.0$ & $45.7 \pm 1.7$ & $48.7 \pm 2.5$ & $*$ & - & - \\
\hline $\mathrm{CD}^{+} \mathrm{T}$ cells $(\%)$ & $58.2 \pm 2.4$ & $57.5 \pm 1.8$ & $60.3 \pm 2.4$ & $58.6 \pm 1.5$ & - & - & - \\
\hline $\mathrm{CD}^{+}{ }^{+} \mathrm{CD} 4^{+} \mathrm{T}$ cells $(\%)$ & $17.8 \pm 1.4$ & $18.4 \pm 1.0$ & $18.6 \pm 1.2$ & $19.1 \pm 1.3$ & - & - & - \\
\hline $\mathrm{CD} 4^{+} \mathrm{CD} 8 \mathrm{a}^{+} \mathrm{T}$ cells $(\%)$ & $13.6 \pm 1.3$ & $9.5 \pm 0.8$ & $10.8 \pm 1.1$ & $9.4 \pm 0.8$ & - & $*$ & - \\
\hline $\begin{array}{l}\text { CD8 }{ }^{+} \text {cytotoxic } T \text { cells } \\
\text { (\%) }\end{array}$ & $24.3 \pm 1.3$ & $23.3 \pm 1.0$ & $22.8 \pm 1.3$ & $22.2 \pm 1.6$ & - & - & - \\
\hline gamma delta T cells (\%) & $25.0 \pm 1.14$ & $28.3 \pm 1.5$ & $28.8 \pm 2.0$ & $29.1 \pm 2.3$ & - & - & - \\
\hline NK cells $(\%)$ & $21.6 \pm 3.2$ & $16.8 \pm 1.8$ & $17.5 \pm 2.2$ & $19.1 \pm 2.8$ & - & - & - \\
\hline B cells (\%) & $14.1 \pm 1.3$ & $13.7 \pm 1.3$ & $14.3 \pm 1.8$ & $14.8 \pm 1.1$ & - & - & - \\
\hline Granulocytes (\%) & $37.6 \pm 2.5$ & $32.2 \pm 2.3$ & $30.7 \pm 2.1$ & $30.4 \pm 2.1$ & + & - & - \\
\hline Granulocyte/lymphocyte & $1.2 \pm 0.2$ & $0.9 \pm 0.1$ & $0.9 \pm 0.2$ & $0.9 \pm 0.2$ & - & - & - \\
\hline Monocytes (\%) & $5.5 \pm 0.3$ & $5.0 \pm 0.3$ & $4.8 \pm 0.3$ & $5.2 \pm 0.3$ & - & - & + \\
\hline SLA-II expression on & $1536.1 \pm$ & $1298.2 \pm$ & $1861.8 \pm$ & $1550.5 \pm 61.7$ & $*$ & $*$ & - \\
\hline monocytes & 129.2 & 64.0 & 171.0 & & & & \\
\hline DC (\%) & $5.2 \pm 0.7$ & $4.3 \pm 0.6$ & $6.3 \pm 1.1$ & $5.9 \pm 0.9$ & $*$ & - & - \\
\hline \multirow[t]{2}{*}{ SLA-II expression on DC } & $17280.5 \pm$ & $16170.9 \pm$ & $15415.3 \pm$ & $19733.8 \pm$ & - & - & + \\
\hline & 1608.4 & 1585.0 & 2096.7 & 2266.4 & & & \\
\hline
\end{tabular}

Significance of housing before the switch (Pre-h), after the switch (Post-h) and their interaction is indicated: * $p<0.05,0.05<+p<0.10$, and non-significant is indicated by " - ". 
Table 2: Means and SEM of the relative frequency of leukocyte subpopulations in pigs exposed to four different housing conditions (B1B2 and E1E2: housed in barren respectively enriched pens throughout the experiment; B1E2 and E1B2: experiencing a change in environment from barren to enriched respectively vice versa from 47 days of age) on day 130 of age, after immunization.

\begin{tabular}{|c|c|c|c|c|c|c|c|}
\hline & B1B2 & B1E2 & E1B2 & E1E2 & Pre-h & Post-h & $\begin{array}{l}\text { Pre-h x } \\
\text { Post-h }\end{array}$ \\
\hline Lymphocytes (\%) & $49.0 \pm 2.6$ & $51.2 \pm 2.4$ & $46.1 \pm 2.3$ & $50.4 \pm 2.4$ & - & $*$ & - \\
\hline $\mathrm{CD}^{+} \mathrm{T}$ cells $(\%)$ & $63.7 \pm 2.2$ & $67.1 \pm 1.2$ & $66.8 \pm 1.7$ & $66.2 \pm 1.8$ & - & - & - \\
\hline $\mathrm{CD}^{+}{ }^{+} \mathrm{CD} 4^{+} \mathrm{T}$ cells $(\%)$ & $12.2 \pm 0.9$ & $10.8 \pm 0.9$ & $12.5 \pm 1.0$ & $12.3 \pm 0.7$ & - & - & - \\
\hline $\mathrm{CD}^{+}{ }^{+} \mathrm{CD} 8 \mathrm{a}^{+} \mathrm{T}$ cells $(\%)$ & $13.6 \pm 1.4$ & $11.6 \pm 1.0$ & $12.9 \pm 1.4$ & $11.5 \pm 1.0$ & - & $*$ & - \\
\hline $\begin{array}{l}\text { CD8 }{ }^{+} \text {cytotoxic T cells } \\
\text { (\%) }\end{array}$ & \multicolumn{6}{|c|}{ (\%) - } & $*$ \\
\hline gamma delta T cells (\%) & $31.2 \pm 1.8^{\mathrm{a}}$ & $36.2 \pm 1.6^{\mathrm{bx}}$ & $34.4 \pm 2.4^{\text {ay }}$ & $32.3 \pm 2.1^{\mathrm{ab}}$ & - & - & $*$ \\
\hline NK cells (\%) & $7.7 \pm 1.0$ & $9.6 \pm 0.8$ & $8.2 \pm 0.8$ & $9.1 \pm 1.6$ & - & - & - \\
\hline B cells $(\%)$ & $10.1 \pm 0.9$ & $8.0 \pm 0.7$ & $9.8 \pm 1.0$ & $9.6 \pm 0.6$ & - & - & - \\
\hline Granulocytes (\%) & $36.9 \pm 2.3$ & $32.6 \pm 2.0$ & $42.4 \pm 2.1$ & $34.5 \pm 2.4$ & + & $* *$ & - \\
\hline Granulocyte/lymphocyte & $0.68 \pm 0.07$ & $0.55 \pm 0.06$ & $0.85 \pm 0.08$ & $0.63 \pm 0.07$ & $*$ & $* *$ & - \\
\hline Monocytes (\%) & $7.9 \pm 2.0$ & $6.1 \pm 0.7$ & $4.8 \pm 0.4$ & $6.0 \pm 0.6$ & + & - & - \\
\hline SLA-II expression on & $1629.8 \pm$ & $1752.3 \pm$ & $2012.1 \pm$ & $1877.3 \pm$ & $*$ & - & - \\
\hline monocytes & 158.2 & 135.7 & 126.0 & 125.7 & & & \\
\hline DC (\%) & $7.5 \pm 0.7^{a b}$ & $6.1 \pm 0.6^{a}$ & $7.2 \pm 0.8^{\mathrm{ab}}$ & $8.0 \pm 0.5^{b}$ & - & - & $*$ \\
\hline \multirow[t]{2}{*}{ SLA-II expression on DC } & $10568.1 \pm$ & $9560.2 \pm$ & $9898.9 \pm$ & $10480.8 \pm$ & - & - & - \\
\hline & 623.6 & 1156.4 & 728.3 & 1139.7 & & & \\
\hline
\end{tabular}

Significance of housing before the switch (Pre-h), after the switch (Post-h) and their interaction is indicated: ${ }^{* *} p<0.001,{ }^{*} p<0.05$, and non-significant was indicated by " - ". In case of interaction effects, pairwise differences are indicated by letter $a$ and $b(p<0.05)$. 



\section{Chapter 8}

General Discussion 



\subsection{Summary}

The objective of this thesis was to investigate the effects of early life and current housing conditions on behaviour, affective states, and immunity in pigs. First, we aimed to investigate whether environmental enrichment influences levels of natural (auto)antibodies in healthy (Chapter 2) and infected (Chapter 3) pigs. Second, to further study the effects of early life enrichment, current housing and a (mis)match between early and later life housing, an experiment was conducted in which pigs were housed in either barren or enriched pens from birth, with half of them experiencing a housing switch at 7 weeks of age, resulting in four treatment groups. Behaviour (Chapter 4), (negative) affective state (Chapter $5 \& 6$ ), and immune competence (Chapter 7) were assessed in pigs from those four groups.

The first two experiments described in this thesis show that environmental enrichment influenced natural (auto)antibody (NA(A)b) levels in both healthy and infected pigs, which may reflect or affect their immune competence (Chapter $2 \& 3$ ). As inconsistent results were found in Chapter 2, 3 and earlier experiments, possible reasons for this discrepancy are discussed in this chapter. To explore the link between $N A(A) b$ levels and disease, correlations between $N A(A) b$ levels, and viral clearance and histological lung scores from the pigs in Chapter 3 are presented in this chapter.

Chapter 4 demonstrates that environmental enrichment profoundly alters the behaviour of pigs, with not only an impact of the current presence or absence of enrichment, but also long-term effects of early life housing experience and a housing switch. In Chapter 5, current, but not early life, housing affected the behaviour in an attention bias test. In Chapter 6, 
irrespective of the current housing conditions, however, pigs originating from a barren pen in early life were more susceptible to reward loss and seemed to have a more negative affective state. Lastly, both early life and current housing conditions, as well as a switch in housing, influenced specific antibodies and leukocyte subpopulations in pigs (Chapter 7). The effects of early life housing and a (mis)match between early life and current housing on behaviour, affective state, and immune competence and responsiveness are discussed separately. Extra data on additional tests not included in the previous chapters are incorporated in the discussion of affective state. Subsequently, the relationships between immune parameters and behaviour in the home pen and in tests of affective state are explored using a factor analysis followed by correlations, as a first step to investigate whether environmental enrichment might (partly) influence immunity via its effect on behaviour and affective state in pigs. Then, the effects of coping style on behaviour, affective state, and immunity found in Chapter 2, 4, 5, 6, and 7 are discussed and lastly the practical implementations of environmental enrichment and suggestions for the future work are given.

\subsection{Enrichment effects on natural (auto)antibodies}

Chapter 2, 3 and 7 indicate effects of environmental enrichment on natural (auto)antibodies and how these change after stress and infection. Natural antibodies (NAb) are defined as antigen binding antibodies without known exposure to this antigen, often with low affinity (Casali and Notkins 1989). NAb are important for very early innate immunity and important as first line of defence against microbial infections due to their poly-specificity (Coutinho 
et al. 1995). They also play a role in clearing apoptotic cells and other waste products (Lutz et al. 2009), and maintain B cell homeostasis (Manson et al. 2005; Quartier et al. 2005). NAb do not only directly protect against infection, but also prime the adaptive immune response by facilitating specific antibody responses (Lammers et al. 2004; Manson et al. 2005). A large part (70-80\%) of the NAb repertoire binds self-antigens, and these antibodies are referred to as natural autoantibodies (NAAb). In this thesis, NA(A)b were studied binding three different model (auto)antigens. Keyhole limpet hemocyanin $(\mathrm{KLH})$ is an antigen without known cross reactivity with mammalian antigens and it has been widely used to detect NAb in a variety of species (e.g. Berghof et al. 2015; Reimert et al. 2014b; Van Knegsel et al. 2007). Myelin basic protein (MBP) is an important protein in the process of myelination of nerves in the nervous system. An association between antibodies binding MBP and stress-related neurological damage has been found in humans, and it has been suggested that these MBP-NAAb act in an homeostatic way in the brain (Abou-Donia et al. 2013). Phosphorylcholine (PC) is a component of mammalian cell membranes and bacterial cell walls which is recognized by IgM autoantibodies after cell damage and inflammation (Kim et al. 2002; Lutz et al. 2009). NA(A)b binding MBP and PC are therefore interesting targets to study effects of both acute and long-term housing challenges on immunity.

$\mathrm{KLH}$ is a very large glycoprotein $\left(\approx 10^{6} \mathrm{KD}\right)$, and the epitopes which are bound by NAb (or even specific antibodies following immunization with $K L H$, as studied in Chapter 7) are not well known. Compared with KLH, MBP ( $\approx 21 \mathrm{KD})$ and PC-BSA $(\approx 70 \mathrm{KD})$ are much smaller molecules. This implies that antibodies binding MBP or PC-BSA are related to a more homogenous group 
of B cells (receptors) whereas KLH antibodies are more likely heterogeneous, and likely binding to many different epitopes. Recently the involvement of Toll-like receptors (TLRs) in the formation of NAb and NA(A)b was indicated in mouse (Kreuk et al. 2019). TLRs recognizing non-self (external) antigens, such as TLR 2 and 4, interact with B cell receptors resulting in other, more specific, antibodies than those produced following activation of TLRs that recognize internal antigens, such as TLR 7 and 9. PC binding antibodies were related to activation of TLRs 7 and 9 (Kreuk et al. 2019) and most likely antibodies to MBP are related to effects of internal TLRs too. Which TLRs are involved in binding epitopes from KLH is unknown.

It has been suggested that $N A(A) b$ production is largely independent of internal or external stimuli (Ochsenbein and Zinkernagel 2000). In Chapters 2, 3 and 7, however, both an effect of a chronic contrast in housing conditions (see Table 8.1), and of acute stressors and infection on NA(A)b titers were found (see Table 8.2). Also others have reported effects of long-term conditions, such as sustained food restriction and social conflict in rats (Cuervo et al. 2018), and barren vs. enriched housing in pigs (Reimert et al. 2014b), on NAb levels. Effects of enrichment on NA(A)b in pigs were not always consistent over studies (see Table 8.1). For instance, when looking at time points without acute stress, environmental enrichment decreased both isotypes binding KLH in Chapter 3, decreased KLH-IgM, and had no influence on KLH-IgG in Chapter 7, and enhanced both natural KLH-IgM and IgG in Reimert et al. (2014b). Also, enrichment effects on antibodies binding MBP and PC-BSA were inconsistent over studies (see Table 8.1). There could be several reasons for these discrepancies, apart from the molecular features of the several antigens discussed above. First, enrichment was applied at 
different stages of life and for different durations, and also blood samples were taken at different ages. Second, the exact type of enrichment varied, as the enrichment in Chapters 3 and 7 did not only include ample substrates to root in as in the study of Reimert et al. (2014b), but also included extra space, which seems to be another important enrichment factor for pigs (Cornale et al. 2015; Vermeer et al. 2014). The set-up of environmental enrichment experiments could have resulted in different antigenic conditions, leading to different effects on $N A(A) b$ levels. It should be noted that even when using the same type of rooting substrates, such as straw, over studies, the hygienic conditions of this material may vary (Tuyttens 2005) and therefore the impact on the immune system too. Hygienic conditions, causing differences in intestinal microbial contents have been found to affect NAb levels. For example, Haghighi et al. (2006) found higher levels of NAb binding KLH in chickens fed with specific probiotics, and, moreover, the hygiene status on chicken farms profoundly affected the levels of NAb in the birds. Finally, the $N A(A) b$ levels measured may have been influenced by (both induced or unintended) challenges the animals encountered before sampling, as we found effects of acute stressors like mixing, transport and relocation on $N A(A) b$ titers which were, moreover, housing-dependent. 
Table 8.1: Effects of environmental enrichment treatments on $N A(A) b$ levels as compared to barren housing conditions in pigs in different studies.

\begin{tabular}{|c|c|c|c|c|c|c|c|c|c|c|}
\hline \multirow[t]{2}{*}{ Study } & \multicolumn{2}{|l|}{ Enrichment } & \multirow[t]{2}{*}{ Age } & \multirow{2}{*}{$\begin{array}{l}\text { KLH- } \\
\text { IgM }\end{array}$} & \multirow{2}{*}{$\begin{array}{l}\text { KLH- } \\
\text { IgG }\end{array}$} & \multirow{2}{*}{$\begin{array}{l}\text { MBP- } \\
\text { IgM }\end{array}$} & \multirow{2}{*}{$\begin{array}{l}\text { MBP- } \\
\text { IgG }\end{array}$} & \multirow{2}{*}{$\begin{array}{l}\text { PC- } \\
\text { BSA- } \\
\text { IgM }\end{array}$} & \multirow{2}{*}{$\begin{array}{l}\text { PC- } \\
\text { BSA- } \\
\text { IgG }\end{array}$} & \multirow{2}{*}{$\begin{array}{l}\text { PC- } \\
\text { BSA- } \\
\text { IgA }\end{array}$} \\
\hline & Type & Period & & & & & & & & \\
\hline [1] & Substrates $^{1}$ & $>$ weaning & $\begin{array}{l}\text { average w8, } \\
9,22\end{array}$ & $\uparrow$ & $\uparrow$ & - & - & - & - & - \\
\hline \multirow[t]{3}{*}{ Ch. 2} & Substrates ${ }^{1}$ & >weaning & w8 & - & - & ns & ns & $\downarrow$ & ns & ns \\
\hline & & & $\begin{array}{l}\text { w9 (3d after } \\
\text { regrouping) }\end{array}$ & - & - & $\uparrow$ & $\uparrow$ & $\downarrow^{+}$ & $\uparrow$ & $\uparrow$ \\
\hline & & & w22 & - & - & $\uparrow$ & ns & ns & $\uparrow^{+}$ & $\uparrow$ \\
\hline Ch. 3 & $\begin{array}{l}\text { Substrates }^{2}+ \\
\text { space }+ \text { social }\end{array}$ & >birth & $\begin{array}{l}\text { d38 } \\
\text { d44 (3d after } \\
\text { transport) }\end{array}$ & $\begin{array}{l}\downarrow^{+} \\
\text {ns }\end{array}$ & $\begin{array}{l}\downarrow \\
\downarrow\end{array}$ & $\begin{array}{l}\downarrow \\
\mathrm{ns}\end{array}$ & $\begin{array}{l}\downarrow \\
\mathrm{ns}\end{array}$ & $\begin{array}{l}\downarrow \\
\text { ns }\end{array}$ & $\begin{array}{l}\mathrm{ns} \\
\uparrow\end{array}$ & - \\
\hline Ch. 7 & $\begin{array}{l}\text { Substrates }{ }^{2},+ \\
\text { space }+ \text { extra } \\
\text { enrichment }\end{array}$ & $\begin{array}{l}>\text { birth } \\
\text { or }>\text { w73 }\end{array}$ & d74 & $\downarrow$ & ns & - & - & - & - & - \\
\hline
\end{tabular}

Higher levels of $N A(A) b$ in environmental enrichment treatments as compared to barren housing are indicated by $\uparrow$, lower levels by $\downarrow$, and $\uparrow^{+}$and $\downarrow^{+}$indicates a tendency in increase and decrease, respectively, and no difference by ns. >birth=after birth; >weaning=after weaning (week 4); -=not measured; $w=$ week; $d=$ day of age. ${ }^{1,2}$ Substrates were ${ }^{1}$ straw; ${ }^{2}$ straw, peat, and sawdust. ${ }^{3}$ Enrichment effect irrespective of whether pigs were housed enriched from birth or w7. [1] refers to study of Reimert et al. (2014b).

The results from Chapter 2 and Chapter 3 suggest that apart from changing with age (Parmentier et al. 2004; Reimert et al. 2014b; Star et al. 2007) and being influenced by housing environments (Reimert et al. 2014b, Chapter 2, Chapter 3, Chapter 7), NA(A)b levels also change in response to acute challenges like temporary (24h) regrouping combined with relocation (Reimert et al. 2014b; Chapter 2) to transport combined with relocation and to infection (Chapter 3, see Table 2 for an overview). In a study on chickens (Hangalapura et al. 2003), acute stress caused by $1 \mathrm{~h}$ exposure to low 
temperature increased the levels of natural antibodies binding LPS. Another study reported a rise in serum anti-brain autoantibodies (neuron-specific enolase and S100 protein) one day after termination of stress from restraint ( 2 hours daily, 10 days) and electric tail shocks (80 shocks daily, 19 days) in rats (Andrejević et al. 1997).

Changes in antibody levels seemed to be different for different NA(A)b, possibly depending on the nature of the stressor. In Chapter 2, natural IgM and IgG binding PC-BSA levels were significantly different between the sample taken before the 24-h regrouping test, during which pigs were placed in a new pen, and 3 days after this test. After regrouping, the pigs started fighting to obtain a new social hierarchy and inflicted skin damage (Camerlink et al. 2013). This might explain why particularly antibodies binding PC-BSA were affected, as PC is recognized by NAAb after cell damage and inflammation (Lutz et al. 2009).

In Chapter 3, all NA(A)b measured had changed at 6 days after transport and relocation to a different research unit as compared with levels before this challenge. The fact that levels of more N(A)Ab changed in Chapter 3 than in Chapter 2, might be due to the fact that in Chapter 3 pigs were sampled 6 days after the challenge instead of 3 days in Chapter 2, allowing for a higher increase of the NA(A)b. Another reason could be that the circumstances were more challenging in Chapter 3 than in Chapter 2. Although regrouping is known to be stressful for pigs, in Chapter 2 it was applied for $24 \mathrm{~h}$ only, in the presence of one familiar pen mate, in a new but similar pen as their home pen, and in the same room. In Chapter 3, the challenge was road transport 
to another research facility with a different hygiene status. However, further research is needed on stress-induced changes in N(A)Ab.

Changes in $\mathrm{NA}(\mathrm{A}) \mathrm{b}$ following the different challenges, including infection, were also found to be housing-dependent (Table 8.2), with frequently more pronounced changes, mostly increases, in enriched housed pigs. For instance, rises in IgM-MBP following infection were higher in pigs with enrichment than in pigs kept in barren pens.

Table 8.2: Effects of different challenges on NA(A)b levels in pigs kept in enriched (E) or barren (B) housing.

\begin{tabular}{|c|c|c|c|c|c|c|c|c|}
\hline study & Challenge & KLH-IgM & KLH-IgG & MBP-IgM & MBP-IgG & PC-BSA-IgM & PC-BSA-IgG & PC-BSA-IgA \\
\hline \multirow[t]{2}{*}{ [1] } & Regrouping ${ }^{1}$ & $\mathrm{E} \uparrow$ & $\mathrm{E} \uparrow \uparrow$ & - & - & - & - & - \\
\hline & & $\mathrm{B} \uparrow$ & $\mathrm{B} \uparrow$ & & & & & \\
\hline \multirow[t]{2}{*}{ Ch. 2} & Regrouping ${ }^{1}$ & - & - & ns & $\mathrm{E} \uparrow$ & $\mathrm{E} \uparrow$ & $E \downarrow$ & $\mathrm{E} \uparrow^{+}$ \\
\hline & & & & & B ns & $\mathrm{B} \uparrow$ & $B \downarrow$ & B ns \\
\hline \multirow[t]{4}{*}{ Ch. 3} & Transport $^{2}$ & $\mathrm{E} \uparrow$ & ns & $\mathrm{E} \uparrow$ & $\mathrm{E} \uparrow \uparrow$ & $\mathrm{E} \uparrow \uparrow$ & $E \downarrow$ & - \\
\hline & & $\mathrm{B} \uparrow$ & & B ns & $\mathrm{B} \uparrow$ & $\mathrm{B} \uparrow$ & $\mathrm{B} \downarrow \downarrow$ & \\
\hline & Infection ${ }^{3}$ & $\mathrm{E} \uparrow$ & ns & $\mathrm{E} \uparrow \uparrow$ & ns & $\mathrm{E} \uparrow \uparrow$ & ns & - \\
\hline & & $B \uparrow$ & & $\mathrm{B} \uparrow$ & & $\mathrm{B} \uparrow$ & & \\
\hline
\end{tabular}

Significant increases and decreases of $N A(A) b$ levels following different challenges are indicated by $\uparrow$ and $\downarrow$, respectively. $\uparrow \uparrow$ and $\downarrow \downarrow$ refer to a higher or lower increase or decrease, respectively, compared to the other housing treatment. $\uparrow^{+}$indicates a tendency. No change in NA(A)b levels is indicated by ns. ${ }^{1}$ Regrouping and relocation for $24 \mathrm{~h}$; antibodies measured just before and 3 days after the test. ${ }^{2}$ Road transport to another facility at 39 days of age; $N A(A) b$ measured 1 day before and 5 days after transport. ${ }^{3}$ Infection with PRRSV at 44 days of age; $N A(A) b$ measured just before and 2, 4, and 8 days after PRRSV infection, which was followed by infection with A. pleuropneumoniae at 52 days of age; $N A(A) b$ measured 1 and 2 days after $A$. pleuropneumoniae infection. [1] refers to study of Reimert et al. (2014b).

An important question is whether NA(A)b levels and changes in NA(A)b levels following challenges may have consequences for health and survival. Some 
studies in commercial laying hens found that high NAb levels, especially IgM binding $\mathrm{KLH}$, at adolescence were related to higher survival (Star et al. 2007; Sun et al. 2011; Wondmeneh et al. 2015). Also, breeding for high KLH-NAb levels in chickens increased their resistance to avian pathogenic Escherichia coli (APEC) infection (Berghof et al. 2019). The results of Chapter 3 suggest that MBP-IgM has a beneficial effect on the course of PRRSV infection, as enriched pigs, which displayed a faster clearance of viral RNA (van Dixhoorn et al. 2016), also showed a stronger increase in MBP-IgM levels (Chapter 3). In support of this, within barren housed pigs, which thus generally showed a lower increase in MBP-IgM than pigs from enriched housing following PRRSV infection, a positive correlation was found between the increase in MBP-IgM and the reduction in viral RNA ( $r=0.66$, Chapter 3$)$.

To further explore whether NA(A)b levels and viral clearance are related, correlations between the levels of circulating $N A(A) b$ just before infection (Chapter 3) and viral clearance, calculated as the area under the curve of viral RNA in serum (data from van Dixhoorn et al. 2016), were assessed. The level of natural MBP-IgG just before infection was negatively correlated with this area ( $r=-0.45$, Figure 8.1). Also, when correcting for the housing effect by correlating residuals, this correlation remained $(r=-0.45, p=0.03)$. Similar to correlations between MBP levels just before infection (day 0), significant correlations were found with the levels $2(r=-0.56), 4(r=-0.48), 8(r=-0.46)$, and $9(r=-0.60)$ days after infection as well (all $p<0.05$, correlations when corrected for housing: $-0.57,-0.48,-0.46,-0.59$, respectively). This indicates a lower viral load in pigs with higher levels of MBP-IgG. Therefore, it is speculated that MBP-NAAb levels, both the circulating levels of the IgG isotype and the IgM antibodies that increased in response to infection, are 
related to the response to PRRSV infection in those pigs. The relationship between antibodies binding MBP and viral load remains to be elucidated. PRRSV can be isolated from brain tissue and induces cellular damage to neurons and neuroglial cells in pigs (Cao et al. 2012; Thanawongnuwech et al. 1997), which might explain why MBP, likely binding damaged neural cells, is related to the course of the PRRSV infection, but the exact underlying mechanism needs further study. No other correlations were found between $N A(A) b$ and viral clearance.

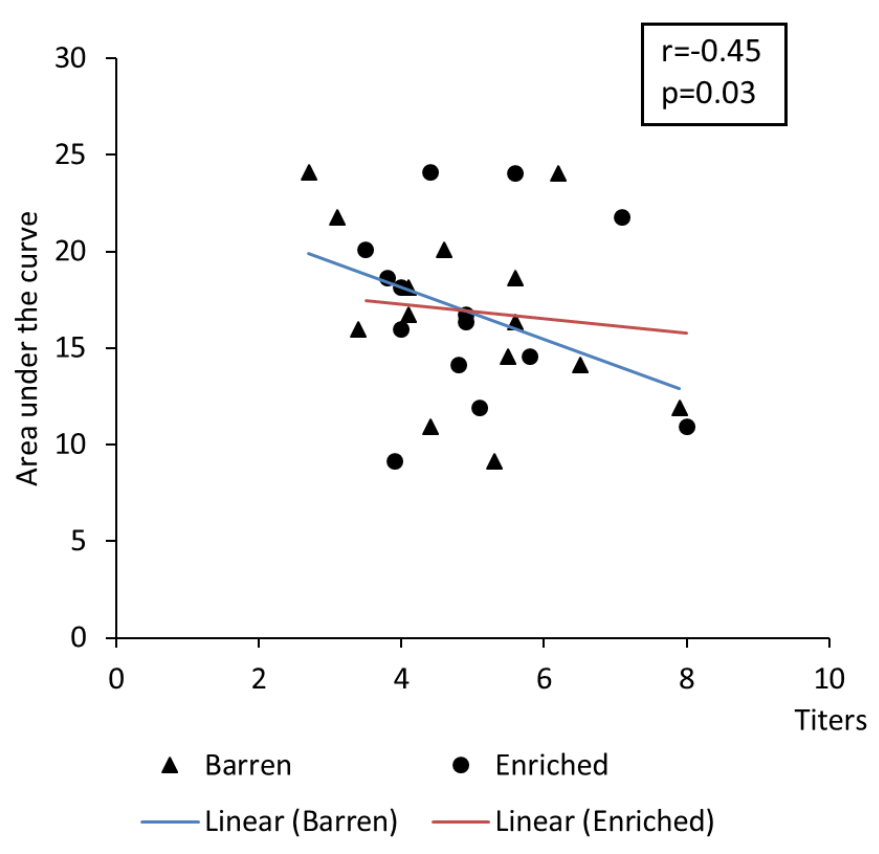

Figure 8.1: Correlation between the levels of natural MBP-IgG just before PRRSV infection and the area under the curve of viral RNA (produced from data of van Dixhoorn et al. 2016) in either barren or enriched housed pigs. 
Correlations between NA(A)b levels (Chapter 3 ) and post mortem lung lesion scores (data from van Dixhoorn et al. 2016) after PRRSV and $A$. pleuropneumoniae infection were also analysed in this thesis to explore whether the $N A(A) b$ are related to the clinic histological symptoms after these infections. There was a correlation between the area under the curve of viral RNA and lung lesion scores $(r=0.40, p=0.06, r=0.52, p=0.01$ for raw correlation and correlation corrected for housing, respectively), i.e. a higher viral load was associated with more lung damage. MBP NAAb were, despite the correlation between the IgG isotype and viral load, not significantly correlated with lung lesion scores. The level of KLH-IgG just before infection was positively correlated with the lung lesion scores ( $r=0.42, p=0.03)$, but when correcting for housing treatment, the correlation was not significant anymore $(r=0.31, p=0.11)$. Similarly, uncorrected KLH-IgG levels at 4 days after infection were also positively correlated with lung lesion scores $(r=0.41$, $p=0.04)$, but not after correction for housing $(r=0.18, p=0.40)$. This absence (or even positive uncorrected correlation) between KLH-lgG levels and lung lesion scores was unexpected given the favourable association between KLH $\mathrm{NAb}$ and survival and disease resistance found in chickens (Berghof et al. 2019; Star et al. 2007; Sun et al. 2011; Wondmeneh et al. 2015). It is also not in line with a recent abstract reporting that pigs reaching market age (i.e. a proxy for survival) had higher IgG NAb binding KLH (but also NAb binding lipopolysaccharides, lipoteichoic acid, and peptidoglycan), and that genotypic high NAb levels were related to fewer disease treatments, lower mortality, higher finishing average daily growth and lower day-to-day fluctuations in feed intake (Chen et al. 2019). No correlations were found between PC-BSA NA(A)b and lung lesion scores. 
To conclude, environmental enrichment affected $N A(A) b$ levels in pigs (Chapter 2, 3, 7), and their changes in response to challenges and infection (Chapter 2, 3). NA(A)b levels and their changes, in turn, may be related to disease characteristics. However, more studies are needed to understand the role of the various $N A(A) b$ and their isotypes in resistance to infectious diseases and disorders to confirm and better understand the current results.

\subsection{Effects of (early life) environmental enrichment on behaviour and affective state}

\subsubsection{Effects of environmental enrichment per se}

In Chapter 4, environmental enrichment reduced manipulative behaviour directed to pen mates, and increased exploration and play behaviours, which is in line with other studies revealing beneficial effects of enrichment behaviour of pigs (e.g. Beattie et al. 2000a; Bolhuis et al. 2005a; Fraser et al. 1991; Oostindjer et al. 2011). This was true from an early age onwards, and these effects of enrichment persisted up till at least 18 weeks of age, when the last behaviour observations were done. Besides, pigs housed in enriched pens seemed better able to cope with the weaning transition, as they gained more weight and had a larger feed intake after weaning (Chapter 4). They also had lower basal levels of cortisol (see Box 2 and section 8.5.1). Moreover, environmental enrichment increased growth up till the last measurement at 19 weeks of age (Chapter 4), and affected the response to a novel object, which was an orange traffic cone, presented for $5 \mathrm{~min}$ in their home pens. Pigs housed in an enriched environment were faster to approach and touch this novel object than pigs housed in a barren environment (Figure 8.2A, B), 
similar to a previous study (Beattie et al. 2000a), whereas the percentage of time spent near the object did not differ (Figure 8.2C). This may suggest reduced fearfulness in enriched pigs.
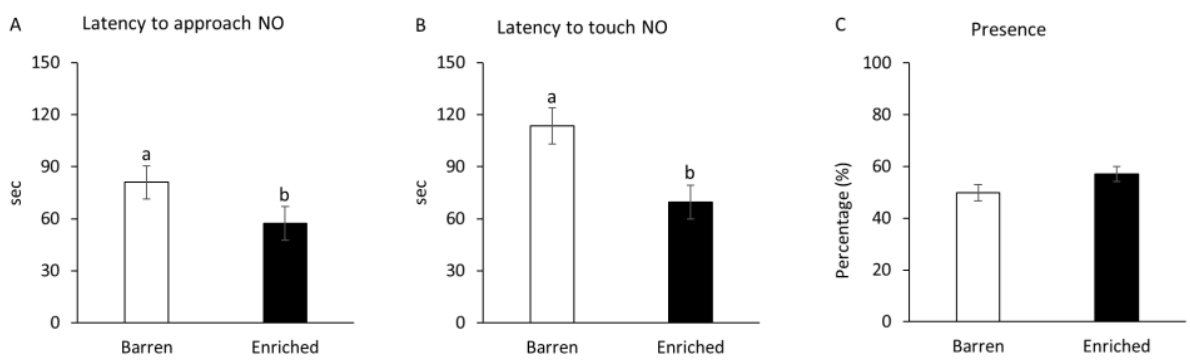

Figure 8.2: Means \pm SEM of the latency to approach within $1 \mathrm{~m}$ of $(A)$ and touch $(B)$ a novel object (NO) and the percentage of time spent near the NO (C) presented for $5 \mathrm{~min}$ in the home pen in pigs housed in either barren or enriched conditions at 43 days of age. Groups lacking a common letter significantly differ $(p<0.05)$.

\subsubsection{Effects of early life environmental enrichment}

It is quite clear that environmental enrichment is beneficial to pigs' welfare based on our findings and those of others, as it better meets the behavioural needs of pigs than barren pens. However, by comparing pigs housed in barren or enriched conditions throughout life, it is unclear whether enrichment effects can be attributed to a more favourable early life development, or to the actual housing situation. Little is known about the putative long-term effects of environmental enrichment in early life, or the impact of a mismatch between early life conditions and the current housing, in pigs.

Early life experiences have been found to modulate the development of behaviour and cognition (review in Telkänranta and Edwards 2018), and alter 
phenotypic, genomic and behavioural expressions of animals. These influences can persist in later life, and therefore have an impact on animal welfare (e.g. Foyer et al. 2013; Kanitz et al. 2004; Latham and Mason 2010). Telkänranta and Edwards (2018) reviewed two different types of process through which environmental conditions in early life can cause lifelong impacts. The first one is phenotypic plasticity as part of normal development. The nature and amount of adversity, such as food availability or threats, in the natural environment of animals vary. This means that the optimal behavioural strategies may be (partly) different for each environment. To adapt to their specific environment, young animals therefore develop different profiles of, for instance, fearfulness, motivation for exploration, boldness and learning. In this way, animals are adaptively adjusted to their environment, providing that this environment is still within the evolutionary boundaries of the species. In line with this, mild stress caused by adverse environments and/or experiences in early life are likely to increase survival and resilience in later life, as the earlier periods of development provide indications and forewarnings of the most likely future conditions (Lewis and Olive 2014). Some studies indeed showed that mild predictable stress early in life may improve certain aspects of health and development. For instance, in rodents, maternal separation (3-15 min/day) during first 2 or 3 weeks of age reduced anxiety and lowered the endocrine response to a mild stressor later in life (Daskalakis et al. 2013; Meaney et al. 1989). I would, however, not define a barren housing environment in early life, as used in this thesis, as a mild stressful event, but more a long-term chronic stressor for pigs. The second process is an inappropriate developmental response in an abnormal early environment. The early environment can be too severely disrupted or 
unnatural for the species in several ways, for instance in case of exposure to traumatic events (e.g. Eid et al. 2019; Lacey et al. 2013; Schmeer et al. 2019) or if parts of the environmental input needed for normal behavioural and cognitive development are lacking. In such cases, animals could be deficient in their adaptive abilities, thereby making them less able to cope with stressful events in later life (review in de Kloet et al. 2005). Therefore, the severity, predictability and controllability of early life events play an important role in determining whether animals would benefit or suffer from their consequences throughout life (Avitsur et al. 2015). Environmental input in the form of enrichment is considered to be important for normal (brain) development in animals, which could also hold for pigs. If enrichment in early life is indeed crucial, then long-term negative effects can be expected if pigs are reared in barren environments lacking suitable stimuli for development. On the other hand, research in other species has shown that enrichment provided in later life can also counteract adverse effects of negative early life experiences on behaviours (Francis et al. 2002; Koh et al. 2005). To investigate whether (lack of) experience with enrichment in early life still has an impact in later life, or can be overruled by the later life environment of pigs, the animals were raised in barren or enriched pens, after which half of them were exposed to a switch to the other environment. Subsequently, the influences of early and the current housing conditions and their combination on behaviour (Chapter 4), affective state (Chapter 5, 6 and data on a novel environment - novel object test presented in this chapter) and immunity (Chapter 7) were assessed. 


\subsubsection{Early life effects combined with current housing effects on behaviour in the home pen}

We found that, even if reared differently, the behaviour of the pigs in Chapter 4 and their basal cortisol levels generally reflected their current environment. Yet, also the early life environment exerted an influence on behaviour, with an effect opposite to that of the current environment. Therefore, behaviours like play and exploration were seen even more in enriched pens if the pigs originated from barren housing, whereas behaviours typically seen in barren pens were more pronounced in pigs from an enriched early life environment. The switch from barren to enriched housing thus even seemed to reveal a 'catching-up' effect, which could reflect a heightened motivation for exploration and play in pigs that had been deprived of enrichment in early life. The results of Chapter 4 show that maladaptive behaviours developed in the barren early life environment in the first 7 weeks of life, such as ear biting or tail biting, did not retain, but could still be reversed by enrichment in later life. Hence, the pigs flexibly adjusted their behaviour to their new environment. It remains to be shown whether this would also hold for pigs exposed to a change in environment at a later age. Even though the peak in brain growth (Dobbing and Sands 1978) and in play behaviour (Spinka et al. 2001) occur at an earlier age, the pigs were still relatively young at the time of the switch.

Pigs in barren housing that originated from enriched pens spent as much or even more time (coping-style dependent, see section 8.5) on damaging behaviours than pigs that had been reared in barren pens, and showed the least play behaviour (Chapter 4). Enriched rearing conditions therefore do 
not seem to protect pigs from developing unwanted behaviours in suboptimal environments in later life. Pigs experiencing enrichment before are likely less motivationally satisfied by barren resources than animals without such high expectation, which is called a 'negative contrast' effect (Pecoraro et al. 1999; Wiegmann et al. 2003). Therefore, loss of enrichment seems frustrating for pigs. It has been found in several other studies too that abnormal behaviours become even more prevalent and severe in animals with enrichment removal than in those without enrichment all their lives. For example, male rhesus monkeys experiencing loss of enrichment had more stereotypic behaviours than before the enrichment was given (Bayne et al. 1992), and removing straw from pigs increased abnormal tail-biting above levels in pigs never provided with straw (Day et al. 2002a).

The 'catching up' and 'frustration' effects in pigs that changed from barren to enriched pens and vice versa, were also reflected in their growth. Starting off with a lower body weight, pigs that switched from barren to enriched pens, i.e. the 'positive contrast' group, grew the most, reaching a similar end weight as pigs kept in enriched pens throughout, and, conversely, pigs that switched from enriched to barren pens, the 'negative contrast' group, lost their advance in body weight. The effect of the (mis)match between early life and current housing could also have impact on the affective state of the pigs, potentially not only short-term but also in the long run. This will be discussed in the next paragraph. 


\subsubsection{Early life effects combined with current housing effects on affective state}

Based on the behavioural findings described above, barren housed pigs were expected to have a more negative affective state than enriched housed pigs, and even more so if they originated from an enriched early life environment and consequently had experienced a downshift in housing conditions. To investigate whether early life and current housing conditions, and a (mis)match between those would have long-term effects on affective state, pigs were exposed to a number of tests. These were a novel environment novel object test (NET-NOT), indicating fearfulness (this chapter, results summarized in Box 1; methods given in Appendix 1), an attention bias test (ABT), indicating anxiety (Chapter 5 ), and a successive negative contrast (SNC) test, indicating 'disappointment' or frustration in response to a downgrade in reward, further referred to as 'reward loss' (Chapter 6).

As shown in section 8.3 (Figure 8.3.1), enrichment reduced fear-related behaviours towards a novel object presented in the home pen (NOT) at 6 weeks of age, before the switch of housing. A second home pen NOT was carried out at the end of the experiment to study (early life) housing and switch effects. Unfortunately, at that age the barren pens were too crowded for all the pigs to approach the novel object. Therefore, the results were not reliable and are not reported here.

Most of the responses of pigs in the other tests of affective state (NET-NOT, $A B T$ and $S N C)$, did not reflect the expected effects of early life and current enrichment and their combination. Moreover, results of the three tests did not all point in the same direction. Briefly, the NET-NOT revealed, before the 
drop of the object, more walking, standing alert, grunting and high-pitched vocalising in pigs from enriched current housing, the latter even stronger so if pigs also originated from enriched pens before the switch (see Box 1). After the drop of the object in the NET-NOT, pigs from enriched current housing still grunted more and were more likely to utter high-pitched vocalisations. Pigs both reared and housed in barren pens spent the least time standing alert. This is not in line with our expectation that barren housed pigs, and particularly the ones that came from enriched housing, would have the most negative affective state. More standing alert and vocalisations may indicate a higher fear level in enriched housed pigs, as standing alert and high-pitched vocalisations are generally suggested to reflect fearfulness (Leliveld et al. 2016; Reimert et al. 2015; Ursinus et al. 2014). Similarly, behaviours following the threat in the ABT suggest, based on increased vocalising and vigilance, higher anxiety in pigs from enriched housing, but particularly in the ones with a proactive coping style (Chapter 5 , see 8.6 for discussion on coping style). The SNC test, in contrast, revealed that pigs with a barren rearing history were more sensitive to reward loss, suggesting they were emotionally less able to cope with a 'disappointment' than pigs from enriched early life housing (see Chapter 6). Furthermore, pigs kept in barren housing throughout life, were slower to approach the reward throughout the training and testing period in the SNC, and this low motivation could be interpreted as a reflection of a negative affective state, i.e. anhedonia. Like in the other two tests, coping style also influenced behaviour in the SNC. Coping style effects will be discussed separately in section 8.6.

There could be several reasons for, firstly, the unexpected effects of early life and current housing on the behaviour of pigs in the tests, and, secondly, for 
the inconsistent outcomes in the different tests conducted. These will be discussed below.

To begin with, the behaviour of pigs in the NET-NOT and ABT may not have reflected fearfulness and anxiety only, but also social motivation and motivation to explore. Enriched pigs seemingly were most frightened in both tests, based on their vigilance (standing alert) and vocalisations. However, they did not show a longer latency to touch the novel object in the NET-NOT, nor a higher cortisol response to this test (see Box 2), which is not in line with a higher stress or fear level. Unlike the home pen fear test, in which enriched pigs responded less fearfully than their counterparts from barren housing, pigs were individually exposed to the NET-NOT and ABT, not in groups. It could be speculated that the behaviour of enriched housed pigs was partly socially motivated, i.e. that more vocalisations and standing alert reflected attempts to seek contact with their pen mates. Vocalisations are on the one hand related to emotional reactivity, reflecting fear and anxiety (Leliveld et al. 2016), but may also be influenced by the motivation to make social contact with group members (Kiley 1972; Leliveld et al. 2016). Also, the behaviour of the barren housed pigs may have been affected by other motivations than fear alone. Barren pigs spent more time on exploring the novel object in the NET-NOT and also explored more in the ABT. This might suggest that they were more motivated to explore their surroundings, possibly related to the poor opportunities to do so in their stimulus-poor pens. Also the longer time to get to the reward in the SNC seen in pigs kept in barren housing throughout life, could be interpreted by a higher drive to explore the test arena, which is in line with other studies (de Jong et al. 1998; Mendl et al. 1997). 
Furthermore, the contrast between the test environment and the home pen of the pigs may have played a role in determining their behavioural response. Enriched pigs may have experienced the (barren) test rooms as less attractive than their stimulus-rich pens in which substrates and toys were provided. Conversely, the test room may have been more attractive to barren housed pigs, as it was relatively spacious and contained novel stimuli. It is often assumed that the response of pigs in fear tests may reflect the putative longterm effect of environmental conditions, such as enrichment (Tönepöhl et al. 2012), but this may not always be the case if the contrast between the housing situation and the test environment is not the same for all treatment groups, as discussed above. Therefore, I would suggest to do such tests in a more familiar environment for the animals, for example in their home pen, or only use tests in which animals have been habituated to the test environment. Recently, home-pen judgement bias tests have been developed for rats (Jones et al. 2018), collecting data on pessimism and optimism in an automated manner, which could be promising for studies on effects of housing conditions on affective states.

Finally, as we discussed in Chapters 5 and 6, the ABT and SNC test were, to our knowledge, used for the first time in pigs, and there are several points for improvement of the test set-ups which have been detailed in these two chapters.

Apart from revealing unexpected effects of housing, the different tests used showed inconsistent results. Whereas the NET-NOT and ABT test mainly indicated effects of current housing, sensitivity to reward loss in the SNC test was influenced by early life enrichment, with pigs originating from enriched 
pens before the switch being less affected by reward loss. This discrepancy in results from the different tests might be due to the fact that pigs were tested at different ages, although the test performed last was the one revealing early life effects (SNC). Additionally, different aspects of affective states were measured in the NET-NOT, ABT and SNC, i.e. fearfulness, anxiety, and disappointment/frustration, which could be influenced in different ways and to different extents by early life and current housing. It also could be related to the issues discussed above, i.e., as argued, behaviour in the ABT and NET-NOT may have reflected the acute effect of isolation and the contrast between the test room and the home pens of the pigs, rather than the long-term effect of housing treatments on affective state. In the SNC test, pigs were habituated to the test room, and trained multiple times per week, so the test arena was not novel, and, moreover, they received a reward in this test. This might also have contributed to finding other effects of housing than in the other tests conducted. A downside of the SNC test, however, is that the motivation of pigs to run for the reward seemed to decline over time (see Chapter 6).

Summarizing, the outcomes of the tests of affective state do not confirm the results on behaviour, growth (Chapter 4) and basal cortisol (see Box 2 and section 8.5.1) which suggest a poorer welfare in barren housed pigs, and, when looking at behaviour and growth, more so in animals that had experienced enrichment in early life and consequently experienced a downgrade in housing. It is hard to draw conclusions on the impact of early life enrichment, current housing and of a switch in housing conditions on affective state in pigs, as the tests used may not have been optimal for different reasons and/or need further adjustment. As indicated in Chapter 1 , 
also other tests used for assessing affective states have their pitfalls. Therefore, more research is needed on the optimal method for this goal, as well as on indicators of positive affective state. 


\begin{tabular}{|c|c|c|c|}
\hline 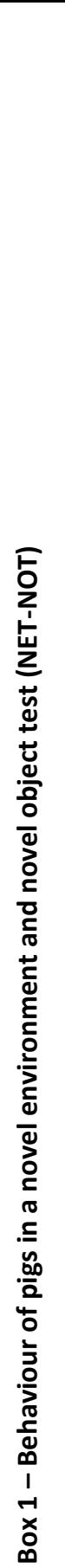 & 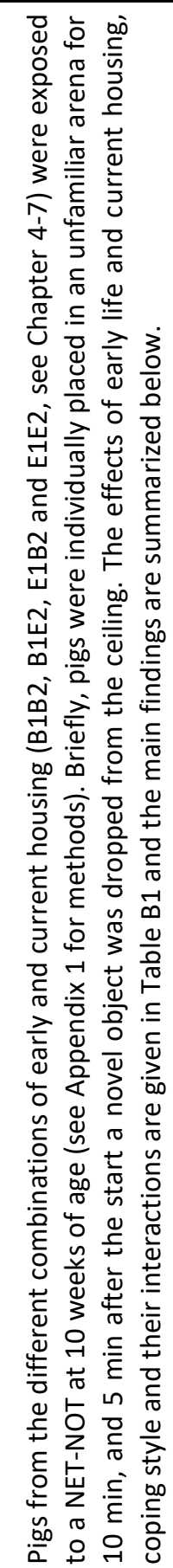 & 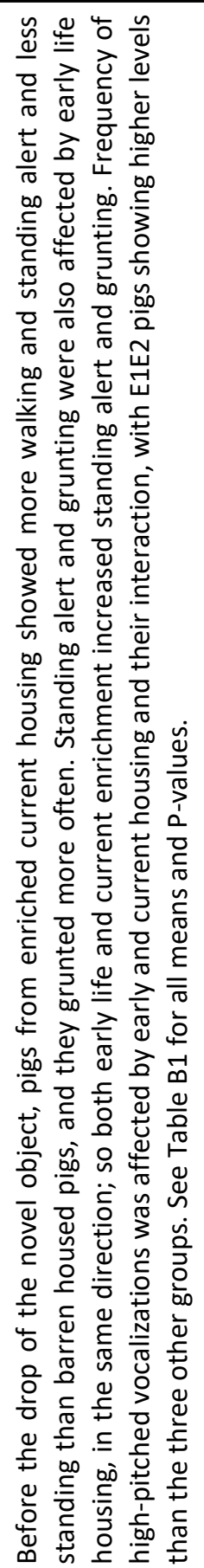 & 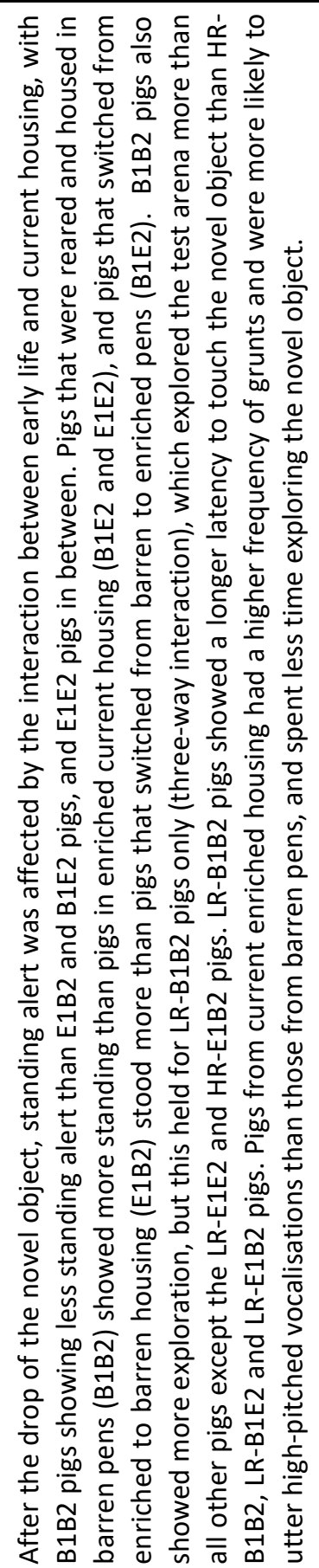 \\
\hline
\end{tabular}




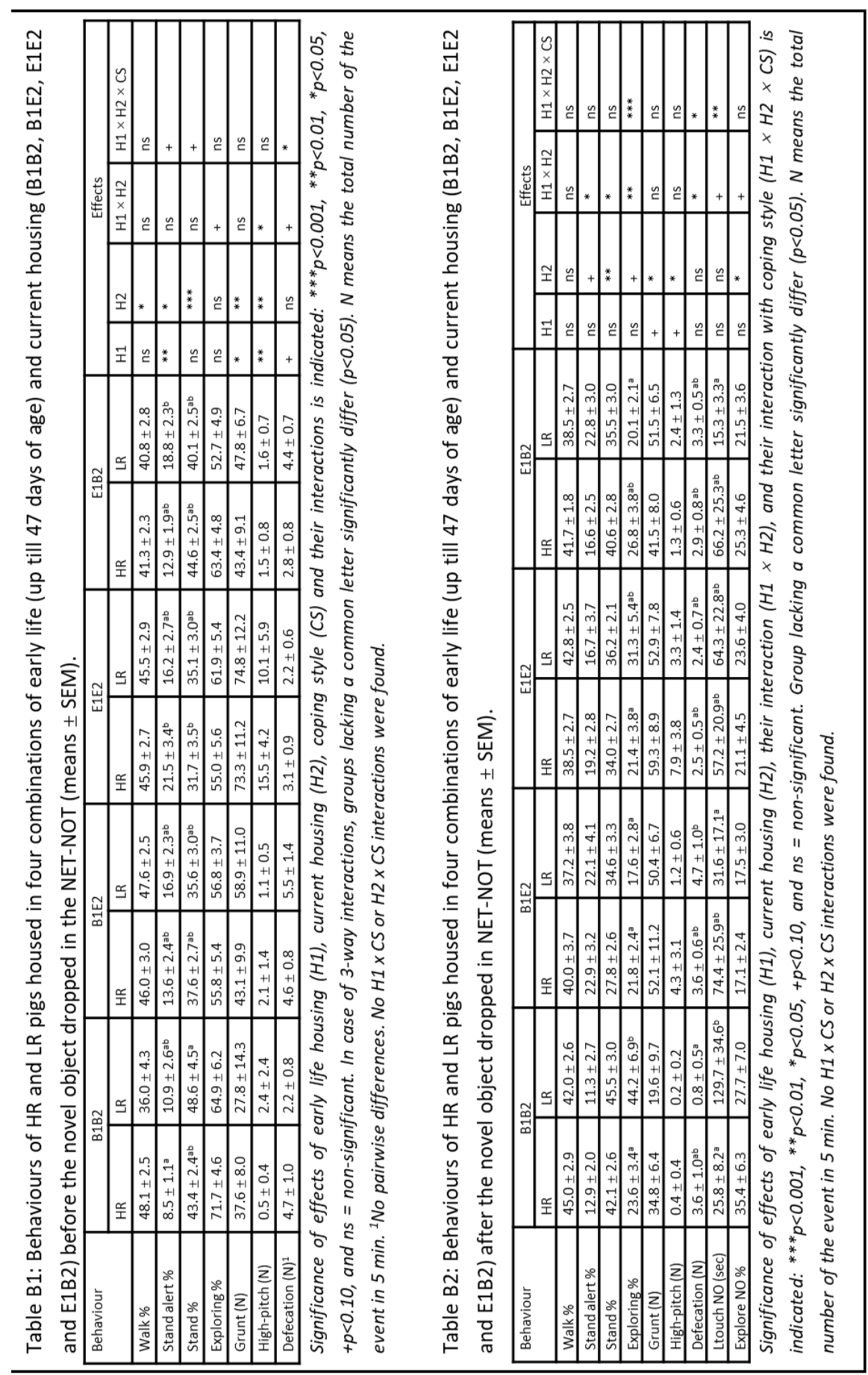




\section{Box 2 - Cortisol response of pigs in a novel environment and novel object test (NET-NOT)}

Pigs from the different combinations of early and current housing (described in Chapter 4-7) were exposed to a NET-NOT at 10 weeks of age (see Appendix 1 for methods). Briefly, pigs were individually placed in an unfamiliar arena for $10 \mathrm{~min}$, and 5 min after the start a novel object was dropped from the ceiling. Saliva samples were taken before $(t=0)$, and 15,30 and 60 min after the test for cortisol analysis.

Cortisol concentrations were higher in pigs from current barren housing than in pigs from enriched housing, both within time points and over all time points $(p<0.05$, Figure B1). The latter effect tended to be strongest in LR pigs (current housing $x$ coping style effect, $p<0.10$ ). Cortisol was also affected by time $(p<0.001)$, and the time $\times$ coping style interaction $(p<0.05)$. Generally, the cortisol concentration increased after the test and declined slower in HR pigs (Figure B2). Post hoc analysis showed that within HR pigs, the cortisol concentration at $t=0$ was lower than at other sampling times $(p<0.05)$, whereas in LR pigs, only the cortisol concentration at $t=15$ was higher than the concentration at $t=60(p<0.05)$. At $t=0$, LR pigs had higher cortisol concentration than HR pigs (LR: $2.47 \pm 0.39, H R$ : $1.72 \pm 0.19 \mathrm{ng} / \mathrm{ml}, \mathrm{p}<0.05)$, and this seems to mainly due to the higher cortisol level in B2 pens, although the interaction was not significant (Figure B2). Cortisol levels at $t=15$ and $t=30$ did not differ for $L R$ and $H R$ pigs, but at $t=60$, LR pigs in E2 pens had lower cortisol level than others ( $p<0.01$, Figure B2).

Figure B1: The cortisol concentration over 4 sampling times just before $(t=0)$ and 15,30 and 60 min after the start of NET-NOT for pigs housed in four housing conditions (B1B2 and E1E2: housed in barren and enriched pens, respectively, throughout life; B1E2 and E1B2: experienced a switch in housing conditions from barren to enriched or vice versa from 7 weeks of age). Significance of early life housing (H1), current housing $(\mathrm{H} 2)$, their interaction $(\mathrm{H} 1 \times \mathrm{H} 2)$, and sampling time (Time) are indicated by $* * * p<0.001$, and non-significance is ns. 


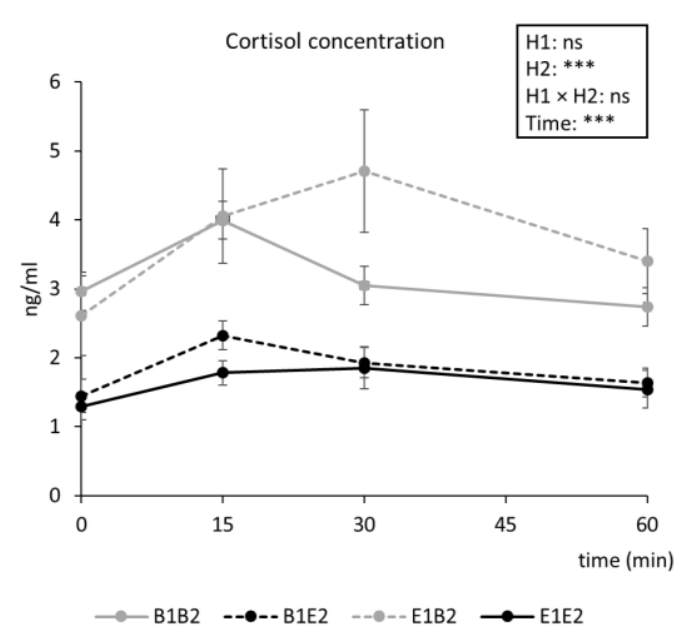

Figure B2: The cortisol concentrations of $H R$ and $L R$ pigs housed in either current barren (B2) or current enriched (E2) housing conditions over four sampling times in NET-NOT. Significance of coping style (CS), the interaction between coping style and current housing $(\mathrm{CS} \times \mathrm{H} 2)$, and the interaction between coping style and sampling time (CS $\times$ time) are indicated by ${ }^{*} p<0.05$, and non-significance is $n s$.

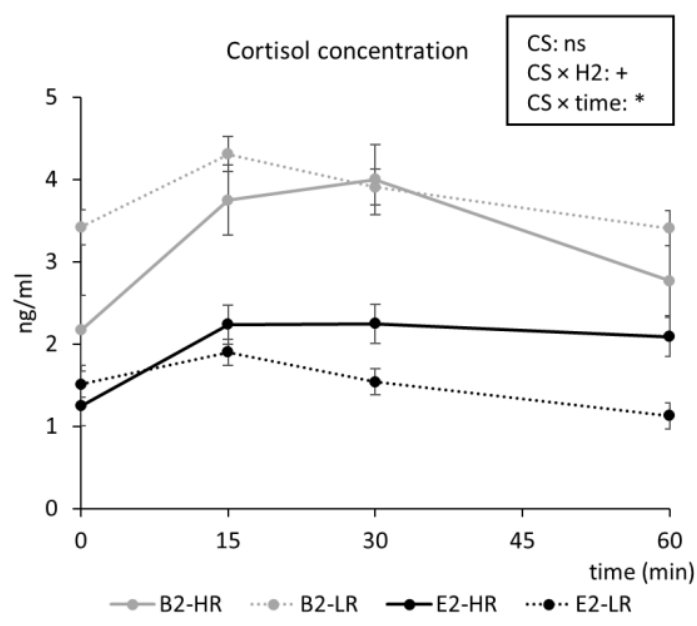




\subsection{Effects of environmental enrichment on innate and specific immunity}

As indicated and discussed above, environmental enrichment affected $N A(A) b$ levels (Chapter 2, 3 and 7), but whether the interplay between enrichment in early life and current housing would affect innate and adaptive immunity in pigs was not known. To investigate this, primary and secondary $\lg \mathrm{M}$ and $\lg \mathrm{G}$ antibody responses and the relative frequencies of peripheral blood leukocyte subpopulations in pigs were studied in Chapter 7.

\subsubsection{Early life effects combined with current housing effects on immunity}

Early life enrichment and/or its combination with current housing exerted effects on specific humoral immune responses (see Chapter 7). Following primary immunization with $\mathrm{KLH}$ at 10 weeks of age and secondary immunization at 15 weeks, KLH-IgG antibody levels were higher in pigs originating from an enriched early life environment than in pigs reared barren pens, irrespective of current housing. KLH-IgM levels were affected by the interplay between early and current enrichment, revealing an effect of the housing switch half of the pigs were exposed to at 7 weeks of age. The KLH-IgM response was higher for pigs kept barren throughout life as compared with pigs reared and kept enriched, and following the switch, the levels of the switched groups moved in the direction of those of pigs kept in their current environment from birth onwards (Chapter 7). Also, the relative frequencies of several leukocyte populations in blood were affected by early life housing, current housing or their interplay. Effects of the early life environment or its interaction with current housing persisted for several peripheral blood leukocyte subsets up to day 130 when the last sample was taken, demonstrating a long-term effect of the pigs' rearing history on their 
immunity. In cases of interactions between early and later life housing on relative frequencies of leukocyte subsets, mostly the pigs kept in barren or enriched housing throughout life did not differ from each other, but one or both of the switched groups deviated from the other groups, implying that the immune system responded to a change in housing conditions.

How exactly the immune system was affected by early and later life enrichment and their interplay is beyond the scope of this thesis. However, it can be speculated that different processes may have played a role. First, contact with and intake of the substrates provided in the enriched pens likely influenced the microbes and nutrients the pigs were exposed to, thereby potentially affecting the immune system. The immune system of pigs moving from barren to enriched pens or vice versa may have responded to this change in antigenic exposure, with apparently quite long-term effects. Moreover, as the oral sampling of rooting substrates and concomitant enlarged antigenic exposure in enriched pens already started in early life, it may have had an impact on intestinal maturation and early microbial colonization, and, consequently, on regulation of innate and adaptive immunity (Mulder et al. 2009; Schokker et al. 2014). This may have contributed to the effect of early housing on the immune variables measured in Chapter 7.

Second, environmental enrichment in early or later life may also have affected the immune system indirectly via its putative effect on stress and mood. As described in Chapter 1, strong bidirectional links between the immune system and the brain allow behavioural and environmental variables to affect immune function and vice versa (Matsunaga et al. 2013). The HPA 
axis plays an important role in linking brain and immune system, and the immunomodulatory effects of stress are mediated at least partly by the release of HPA hormones, such as cortisol (Avitsur et al. 2015; Glaser and Kiecolt-Glaser 2005; Koh 1998). In the novel environment - novel object test (NET-NOT, this chapter), pigs housed in current barren pens had higher salivary cortisol concentrations over the four sampling times, including the baseline level before the test, than pigs kept in current enriched pens (Figure B2 in Box 2). These increased baseline cortisol levels in pigs kept in barren pens are line with other studies ((Reimert et al. (2014a; van de Weerd and Day (2009), but see de Jong et al. (1998) \& de Jong et al. (2000) who found the opposite) and confirm changes in HPA axis functioning due to chronic barren housing conditions in pigs. Given the effects of cortisol on several components of immune system (Chrousos 1995; de Groot et al. 2000), it can be speculated that these changes may have been involved in the effects of current housing on immune status and response (Chapter 2, 3 and 7), but exactly how and to what extent is, however, unknown.

Also, the effects of early life enrichment and its interaction with current housing on immune variables may be explained by changes in brain-immune connections, which, when occurring during early development, may have long term consequences. Numerous studies have demonstrated that stress in early life can reprogram the development of the HPA axis, resulting in lifelong changes in neurotransmitter systems and brain structures (Avitsur et al. 2015; Kaufman et al. 2000). In turn, by affecting brain-endocrine-immune connections, immune function in later life could be influenced as well. For example, repeated social isolation during early postnatal life in piglets caused changes in immune regulation and seemed to produce long-term effects on 
the immune-brain circuitry (Kanitz et al. 2004). Also early life enrichment with substrates (wood shaving and chopped straw) from birth to 9 weeks of age had effects on basal level of cortisol at least until 21 weeks of age (Munsterhjelm et al. 2010). We found, however, no early life housing effect on (basal) cortisol levels as measured in the NET-NOT. Yet, early life housing effects at other levels of the HPA axis cannot be excluded, as well as on other mediators of the brain-immune connection.

In humans, numerous studies have suggested that affective states, such as depression, can be linked to the immune system (Leonard and Song 1999), for example, elevated blood levels of the pro-inflammatory cytokines IL-1 $\beta$, TNF-a and IL-6 are found in depressed patients (Berk et al. 1997; Connor and Leonard 1998; Hestad et al. 2003; Maes 1999). A study in humans also showed that sadness increased and neutral mood reduced IL-18, which is an important regulator of peripheral immune functions, including synthesis of a variety of inflammatory factors that are elevated in depressive illness (Prossin et al. 2016). It has also been reported that individuals with a more negative affective state poorly mount immune responses and may be at higher risk for illness than those with a positive affective state (reviewed in Barak 2006). It should be noted that in the link between affective state and immunity, cause and effect are sometimes difficult to disentangle. Apart from affective state influencing immunity, immune status also can reversely, influence psychology. For instance, depressive symptoms are induced following the therapeutic administration of the IL-2 or IFN-a in humans (Raison et al. 2009). 
In this thesis, we could not disentangle whether (early) enrichment effects on immunity were mediated by its effect on antigenic exposure, and/or via its influence on stress and mood. To explore whether the behaviour and/or affective state of pigs might be related to their immunity, a correlational analysis was done as described in the section below.

\subsubsection{Exploration of putative links between behaviour, affective state and immunity}

As discussed, it can be speculated that the effects of (early life) environmental enrichment on immune competence and responsiveness are not only mediated via a different antigenic exposure as compared with pigs in barren housing, but also via the effects of environmental enrichment on the affective state and behaviour of the pigs. To disentangle these processes and study the impact of the latter on immunity in pigs, more research is needed in which, for instance, antigenic exposure is equal for pigs diverging in affective state. In this chapter, however, it is explored whether the immune status or response of pigs (Chapter 7 ) is related to their behaviour, both in their home pens (Chapter 4) and in a test of affective state (the NETNOT, Box 2 and Appendix 1), and to their growth (Chapter 4).

To reduce the number of variables, factor analysis (FA) was used for the immune variables, behaviours from home pen observations, and behaviours in the NET-NOT separately (see Appendix 2 for details on methodology). Early and current enrichment, and their interaction had strong effects on several of the variables. Therefore, to correct for these effects, residuals of the variables produced in a general linear model were used. All residuals of the variables were entered into a FA with orthogonal varimax rotation and 
factors with an eigenvalue above one were retained. The FA of immune parameters resulted in 8 factors (Table 8.3), and the FA of NET-NOT resulted in 4 factors (Table 8.4). The FA of home pen behaviours only resulted in one factor, therefore, residuals of the separate behaviours, expressed as proportion of time spent on these behaviours averaged over all observation days after the switch, were used in further analyses.

The scores of each animal for each factor produced from the FA on immune parameters were subsequently correlated with the factor scores from the FA on the NET-NOT, and with the behaviours and growth. Here, only the strongest correlations with $p<0.01$ are discussed (see Table 8.5 for all correlations and their significances).

Immune factor 1 (I-F 1) had high (negative and positive) loadings for several types of T-cells on both sampling days, with the exception of T-helper cells (Table 8.3). This factor negatively correlated with proportion of time spent on play behaviours in the home pen over all observation days. Please note that variables were corrected for housing, and therefore play behaviour here represents the individual variation in time spent on this behaviour irrespective of housing. Play behaviour is considered to be associated with positive affect (Fraser and Duncan 1998; Spinka et al. 2001), seems to occur exclusively when the basic needs of animals are met and when they are healthy, and is seen as a typical sign of pleasure (Boissy et al. 2007). Besides, play behaviour is more often observed in animals with low fearfulness (Malmkvist and Hansen 2002). Therefore, immune factor 1 seems negatively associated with time spent on a behaviour that may reflect a positive affective state, but how and why this factor with mostly T-cell features 
relates to play is not known. Interestingly, a recent study found play behaviour to be predictive of a more favourable outcome of a lung disease (Van Dixhoorn et al., in preparation), which might suggest that the relationship found here is not merely coincidental. However, factors obtained from the NET-NOT, part of which may reflect negative affect, did not correlate with immune factor 1.

Immune factor 2 had a high positive loading for relative frequencies of lymphocytes and dendritic cells in peripheral blood on the last sampling day (after the secondary immunization), and a high negative loading for granulocytes. This factor correlated negatively with growth of the pigs following the secondary immunization (Day $109-130$ ). This could be due to the negative influence of the activation of the immune system, which is usually regarded as energetically costly, on the growth during this period. Why a positive correlation between immune factor 2 and chewing was found is difficult to explain. It might due to variation in antigenic exposure caused by oral sampling and chewing of substrates and/or the environment.

No correlations with $p<0.01$ were found between the other immune factors and behavioural variables or growth. Also, the factors obtained from FA of the NET-NOT did not correlate with the immune factors.

In conclusion, an association was found between play behaviour and an immune factor representing T-cells, and a second immune factor reflecting distribution of some immune cells after immunization related to chewing and growth. It has to be kept in mind that this is, as a first attempt to link our immunological and behavioural data, an explorative approach with several methodological drawbacks, including the use of a low number of animals in 
relation to the number of variables. Also, given the many relationships tested, coincidental correlations cannot be excluded. Further studies are therefore needed on the potential links between behaviour, affective state and immunity in pigs.

Table 8.3: Loadings* on the factors with an eigenvalue above one that were extracted by factor analysis with orthogonal varimax rotation on the antibodies before immunizations, the responses of the antibodies during primary and secondary immunizations, and the leukocyte subpopulations before and after immunizations. 


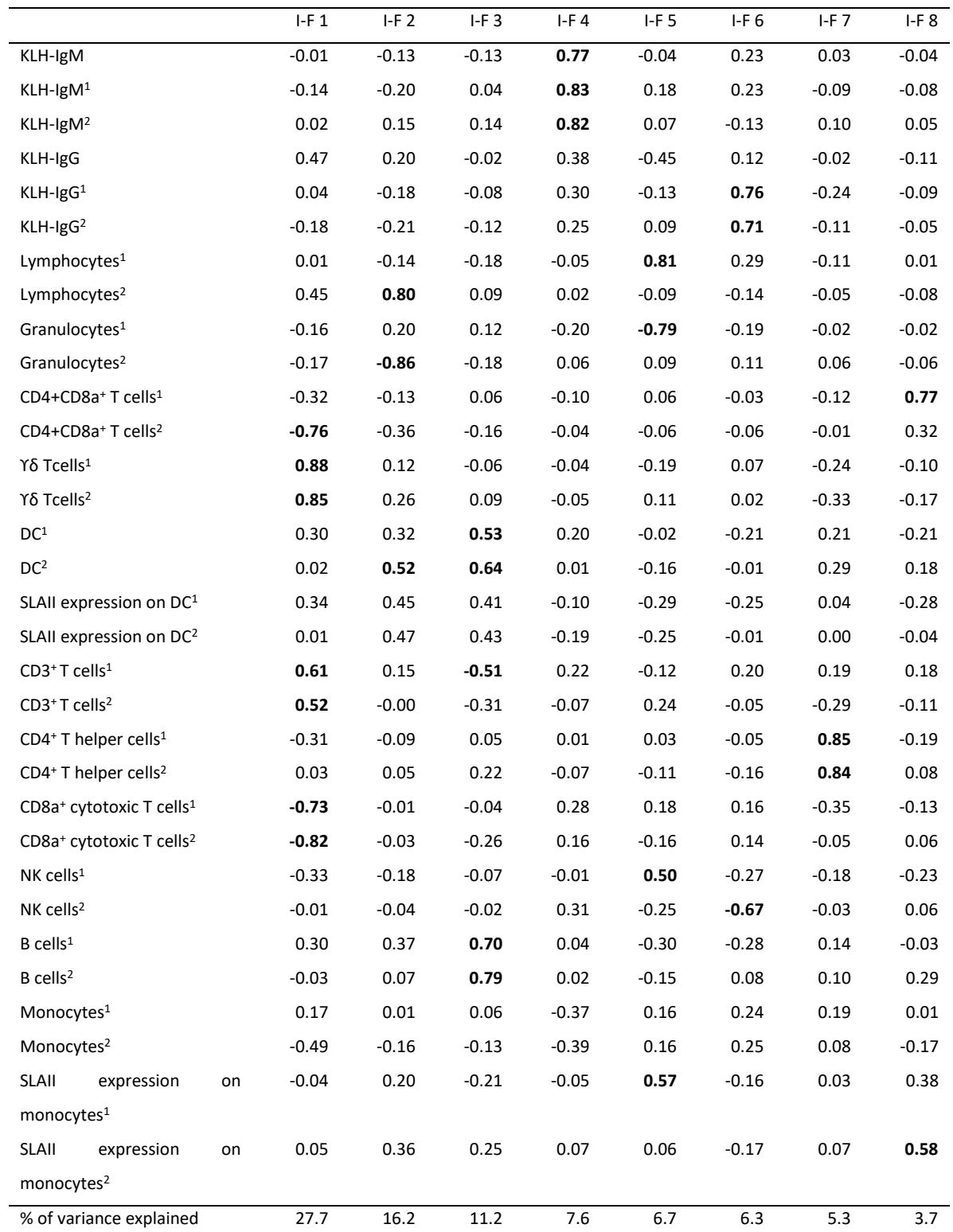

*High loadings (loading $\leq-0.50$ or $\geq 0.50$ ) are indicated in bold. The superscripts 1 and 2 for antibodies mean the area under the curve (antibody response) during primary and secondary immunization, respectively; the superscripts 1 and 2 for cells mean the percentage of the cells before and after immunizations, respectively. 
Table 8.4: Loadings* on the factors with an eigenvalue above one that were extracted by factor analysis with orthogonal varimax rotation on the variables recorded during the novel environment and novel object test (NET-NOT) in the 5min period before the novel object (NO) was dropped and in the 5-min period thereafter.

\begin{tabular}{lcccc}
\hline & F-F 1 & F-F 2 & F-F 3 & F-F 4 \\
\hline Walking $^{1}$ & 0.06 & 0.16 & $\mathbf{0 . 8 7}$ & -0.18 \\
Walking $^{2}$ & 0.25 & 0.26 & $\mathbf{0 . 5 9}$ & 0.07 \\
Standing $^{1}$ & -0.19 & -0.01 & -0.03 & $\mathbf{0 . 8 4}$ \\
Standing $^{2}$ & -0.17 & $\mathbf{0 . 6 6}$ & 0.06 & $\mathbf{0 . 5 3}$ \\
Standing alert $^{1}$ & $\mathbf{0 . 7 0}$ & 0.25 & -0.31 & -0.14 \\
Standing alert $^{2}$ & $\mathbf{0 . 6 4}$ & -0.15 & -0.09 & -0.006 \\
Vocalisations $^{1}$ & $\mathbf{0 . 6 8}$ & 0.15 & 0.19 & -0.12 \\
Vocalisations $^{2}$ & $\mathbf{0 . 8 0}$ & 0.04 & 0.24 & -0.21 \\
Exploring wall and floor $^{1}$ & -0.36 & -0.12 & $\mathbf{0 . 6 1}$ & 0.25 \\
Exploring wall and floor $^{2}$ & -0.07 & -0.49 & 0.07 & $\mathbf{0 . 6 5}$ \\
Exploring bucket $^{\text {Latency to touch bucket }}$ & -0.04 & $\mathbf{0 . 8 5}$ & 0.15 & -0.20 \\
\hline \% of variance explained & -0.35 & -0.73 & -0.15 & 0.12 \\
\hline
\end{tabular}

*High loadings (loading $\leq-0.50$ or $\geq 0.50$ ) are indicated in bold. The superscript 1 and 2 mean the 5-min period before and after the NO was dropped, respectively. 
Table 8.5: Pearson correlation coefficients ( $r$ ) between immune factors (I-PC) and the behaviours in home pen observations (HPO), growth in different periods after housing switch, and factors from the novel environment-novel object test (F-PC), $n=56$.

\begin{tabular}{|c|c|c|c|c|c|c|c|c|}
\hline & I-F 1 & I-F 2 & I-F 3 & I-F 4 & I-F 5 & I-F 6 & I-F 7 & $1-F 8$ \\
\hline Inactivity & 0.15 & -0.05 & -0.15 & -0.08 & -0.23 & -0.19 & -0.14 & 0.09 \\
\hline Aggression & -0.11 & -0.06 & -0.11 & 0.22 & -0.16 & 0.17 & -0.05 & -0.15 \\
\hline Social behav. & -0.05 & -0.10 & 0.14 & -0.21 & -0.04 & 0.19 & -0.07 & 0.17 \\
\hline Play & $-0.34 * *$ & -0.10 & -0.09 & -0.04 & 0.03 & 0.24 & 0.11 & -0.04 \\
\hline Exploring & -0.15 & -0.13 & -0.11 & -0.06 & $0.28 *$ & 0.26 & -0.04 & -0.10 \\
\hline Chewing & -0.03 & $0.43^{* *}$ & 0.10 & 0.04 & -0.01 & 0.07 & $0.34 *$ & -0.04 \\
\hline Manipulation & -0.01 & -0.16 & 0.19 & 0.02 & -0.01 & 0.02 & 0.10 & -0.02 \\
\hline G50-46 & 0.19 & 0.10 & 0.09 & -0.04 & -0.11 & $-0.29 *$ & 0.04 & -0.06 \\
\hline G74-50 & $-0.28 *$ & $-0.32 *$ & $-0.30^{*}$ & -0.16 & 0.18 & $0.33^{*}$ & -0.18 & 0.07 \\
\hline G109-74 & 0.23 & $0.33^{*}$ & 0.14 & -0.24 & -0.08 & -0.05 & 0.16 & -0.24 \\
\hline G130-109 & -0.00 & $-0.53^{* *}$ & -0.09 & 0.02 & 0.08 & 0.20 & -0.00 & $-0.28 *$ \\
\hline G130-46 & 0.05 & -0.15 & -0.07 & -0.23 & 0.04 & 0.17 & 0.03 & $-0.27 *$ \\
\hline F-F 1 & 0.08 & -0.03 & 0.12 & 0.07 & 0.10 & 0.18 & 0.01 & 0.03 \\
\hline F-F 2 & 0.03 & -0.22 & 0.04 & 0.24 & 0.21 & -0.05 & 0.23 & 0.20 \\
\hline F-F 3 & 0.15 & 0.04 & 0.03 & -0.13 & -0.10 & $-0.28 *$ & -0.20 & -0.05 \\
\hline F-F 4 & -0.14 & -0.10 & -0.14 & 0.05 & -0.18 & -0.18 & -0.03 & -0.03 \\
\hline
\end{tabular}

Significant correlations are indicated: ${ }^{*} P<0.01$, and ${ }^{*} P<0.05$, but only ${ }^{*} P<0.01$ were discussed in the text.

\subsection{Coping style effects}

Although not the main focus of this thesis, where possible we balanced for coping style within our treatment groups by pragmatically splitting our population into high resisters (HR) and low resisters (LR) based on their tendency to show an active or passive behavioural response in the backtest, a restraint test in supine position, in early life (Chapter 2, 4, 5, 6, 7). This was done as several studies, often using the more extreme pigs at either end of the population, have revealed differences in stress-induced behaviour, neuroendocrine parameters and gene expression in both types of pig (e.g. 
Gley et al. 2019; Kanitz et al. 2019; Krause et al. 2017; Oster et al. 2015), as well as in immune parameters (e.g. Reimert et al. 2014b; Schrama et al. 1997). Such coping styles, characterized by differences in behaviour and neurobiology, have been described for a wide number of species (review in Koolhaas et al. 2007), and LR and HR pigs seem to resemble what is usually referred to as 'reactive' and 'proactive copers', respectively. Proactive copers are more prone to show an active, 'fight-flight' response to acute stress, whereas reactive copers tend to respond more passively, are more cautious and more flexible in their behaviour (Bolhuis et al. 2004; Bolhuis et al. 2005b; Coppens et al. 2010; Koolhaas et al. 1999).

In Chapter 4, LR pigs were found to be more influenced by their housing environment and by the housing switch, especially the loss of enrichment, as LR pigs that experienced a downgrade in housing from enriched to barren pens spent more time on pen-directed chewing and oral manipulation of pen mates than their HR counterparts. This is in line with other studies which showed that reactive pigs were more influenced by the presence or absence of enrichment, both concerning their behaviour, humoral immune response and the occurrence of gastric lesions (Asher et al. 2016; Bolhuis et al. 2005a; Bolhuis et al. 2006), as well as their affective state (optimistic vs. pessimistic, Asher et al. (2016)). LR pigs were found to have a higher motivation to explore their surroundings than HR pigs (Jansen et al. 2009), which could be related to their tendency to show more manipulative behaviour directed at pen and pen mates when substrates are not available (Bolhuis et al. 2005b) or when they have experienced a loss of enrichment (Chapter 4). Several behaviours in the NET-NOT, especially after the drop of the bucket, were influenced by the three-way interaction between early life housing, current 
housing and coping style (See Table B2 in Box 1). For all three behaviours affected, i.e. latency to touch the novel object, exploration of the test room and frequency of defecation, there were no effects of (early life) housing on HR pigs, whereas the behaviour of LR pigs varied depending on the combination of their rearing and current housing environment. For instance, LR pigs from barren rearing and housing spent more time exploring the test room than several other groups, defecated the least, but were much slower to touch the novel object than barren LR housed pigs that originated from an enriched early life environment. Although it is difficult to exactly explain these behavioural differences, these results do confirm that LR pigs may be more affected by absence or presence of enrichment than HR pigs. This is further supported by the tendency for an interaction between current housing and coping style on (basal) cortisol concentrations, showing that the housing effect seems more pronounced for LR pigs (Figure B2 in Box 2).

In Chapter 5, however, responses of HR pigs in the attention bias test were more influenced by their environment. HR pigs from current enriched housing were found to pay more attention towards (location of) the threat than enriched housed LR pigs, and had the highest levels of walking and squealing after removal of the threat in the attention bias test. As discussed before, the behaviour in the attention bias test may have reflected the acute response to the test setting, involving relocation and social isolation, rather than the long-term effect of housing condition on affective state. This might explain why (enriched) HR pigs were most affected, since they seem to show more difficulty in coping with relocation and social isolation than LR pigs (Geverink et al. 2004; Ruis et al. 2001). In line with this, HR pigs showed a 
slower recovery in cortisol levels than LR pigs after the NET-NOT (Figure B2 in Box 2).

In Chapter 6, LR pigs had a lower latency to get to the reward than HR, but it is difficult to say whether this effect was due to a difference in how LR and HR pigs valued the reward and were motivated for the task, or reflected a difference in balance between different motivations (e.g. explore the runway vs. go for the reward immediately). Concerning the latter, the shorter latencies of the LR pigs are not in line with the general findings that LR pigs explore for longer, even in a familiar test room (Jansen et al. 2009).

No coping style effect was found on NA(A)b levels in Chapter 2 and 7 (coping style was not included in Chapter 3), which is not in line with a previous study, suggesting that HR pigs may have a more active innate immune system (Reimert et al. 2014b). In Chapter 7, LR pigs had a higher level of specific IgM binding KLH than HR pigs, which confirms a higher specific humoral immune response in LR pigs (Hessing et al. 1995; Schrama et al. 1997). The impact of challenges on the immune system of LR pigs might be bigger, as a regrouping test had a more substantial effect on alternative pathway complement activity in LR pigs (Reimert et al. 2014b), but we found no coping style effect on $N A(A) b$ changes after regrouping in Chapter 2. In this thesis, coping style did not affect leukocyte distribution to a large extent (Chapter 7), as only subtle difference were found, but this still further confirms immune differences between the two types of pig found in studies with a more strict selection of extremes at the end of the population (Hessing et al. 1994; Kanitz et al. 2019; Oster et al. 2015; Schrama et al. 1997). Considering the limited number of pigs in Chapter 7, interactions between (early life) housing and 
coping style were not investigated, but it cannot be excluded that differences in immunity between HR and LR pigs may also depend on housing conditions or early life history, which can be studied in the future.

To conclude, results of this thesis confirm that the coping style of pigs may affect behavioural, affective, and immune responses in pigs, and influence how pigs respond to (changes in) environmental conditions. It is therefore important for future studies to keep these influences of individual characteristics of pigs in mind, and, if possible, balance treatment groups for personality traits.

\subsection{Implications and future work}

\subsubsection{Implications of this study}

In this thesis, environmental enrichment was found to influence not only behaviour, but also affective state, growth and various parameters of immunity in pigs. Environmental enrichment for pigs may therefore turn out to be profitable not only by reducing damaging behaviours, but also by improving their mental and physical health. This may contribute to improved longevity and productivity of pigs and reduced medicine use. Besides, the experience of environmental enrichment in early life has long-term effects in pigs, and the mismatch of the early life and current environments also plays an important role in behaviour, affective state and immunity. Switching from enriched to barren conditions seems to be more detrimental for pigs' behaviour. However, the switch did not influence the immune parameters in a straightforward way: the effects on leukocyte subpopulations did not show 
a clear difference between switching from barren to enriched and switching from enriched to barren, suggesting that the immune system is alerted by the changes in the environment in general.

Environmental enrichment is a very broad concept, and a problem with the EC Directive on the provision of appropriate environmental enrichment for pigs is that its leaves much room for interpretation. Environmental enrichment has been studied for several decades, but the actual choice of enrichment to provide to pigs is often arbitrary, based more on economic or health-related factors, than on the requirements of the animals themselves, and as a consequence, pigs rapidly lose interest in the materials applied, making them ineffective (Day et al. 2002b). Therefore, proper enrichment is quite important on commercial farms.

In this thesis, the environmental enrichment used and discussed consisted of rooting substrates (straw, peat, and sawdust), extra space allowance, rotating enrichment objects, and extra enrichment (such as jute sacks, egg trays, branches, raisins) provided once a week, in Chapter 4, 5, 6, and 7; of substrates (sawdust and straw bedding) in Chapter 2; and of substrates (peat, straw and branches) plus extra social experiences in early life in Chapter 3. It is not known whether the effects found in this thesis would hold for all types of enrichment, since different mechanisms are involved. The enrichment used in this thesis was quite 'extreme' compared with the materials that are provided on most farms, and not easily applicable in current housing systems. An important question is whether less elaborate enrichment (e.g. some handfuls of straw or other organic material provided daily or routinely) may yield similar effects. The mandatory enrichment requirement from EU is that 
pigs must have a sufficient quantity of substrates and/or objects to root or explore. Substrates (e.g. straw, peat, sawdust) have been found to positively influence pigs' behaviour (e.g. Beattie et al. 2000b; Petersen et al. 1995), but are, however, more likely to cause immune alterations, since they bring about more antigenic exposure, which can be unfavourable from an hygienic point of view (Tuyttens 2005). Object-enrichment, which is, in spite of the EU Directive, still commonly applied, seems to less efficient than substrates to meet the behavioural needs of pigs and pigs can lose their interest in the objects very soon (review in van de Weerd and Day 2009). Also, we noticed that at the end of the experiment, space allowance could be of increasing importance for finishing pigs, as at an older age they were less active and spent less time on substrate-related behaviours, but needed more space to rest and for other activities, such as walking and feeding. The limited space allowance in barren housing conditions could, on top of a lack of proper materials for exploration, be a reason for damaging behaviours like tail biting, tail lesions and decreased body weight gains in barren housed pigs. Therefore, depending on which stage of life the pigs are in, it may be important to apply different types of enrichment in practice.

In commercial pig farming, piglets will usually be moved to a nursery at weaning and are then called weaners, and when they are around $25-30 \mathrm{~kg}$, they will be transferred to a rearing unit where they stay until slaughter. Each production stage typically takes place either in different units of a farm, or on another farm specialised in the specific production period. Therefore, pigs undergo changes in their (social) environment, need to adapt to this new environment and may experience the loss of the resources and/or social relationships they had before. The housing switch and mixing of unfamiliar 
animals leads to aggressive behaviour (Coutellier et al. 2007), which is a common problem in intensive pig production, potentially resulting in injuries (Gonyou et al. 1988), elevated cortisol levels (Coutellier et al. 2007; Moore et al. 1994), and negative effects on productivity (Stookey and Gonyou 1994; Tan et al. 1991). Especially during weaning, pigs are exposed to the stress of many simultaneous changes at an early age, including maternal separation, an abrupt change in feed source, transportation and handling, and social stress from regrouping with piglets from other litters (review in Campbell et al. 2013). Apart from a difference in physical and social surroundings, altered exposure to dietary and environmental antigens could be another big challenge for their health and welfare. A possible way to reduce stress at weaning, is keeping the same environmental enrichment before and after weaning, or providing extra environmental enrichment after weaning, apart from increasing weaning age. As we found in chapter 4, pigs housed in environmental enrichment in early life were better able to cope with weaning transition as they ate and grew better after weaning, and early life environmental enrichment had rather long-term beneficial effects on welfare, as long as it was not removed at a later age. It could be more detrimental for pigs if they lose the (enrichment) resources they had before and/or have limited space for resting, feeding and growing and establishing a dominance hierarchy, thus it seems to be very important to consider the housing conditions after any change in environment. We only studied a change in housing conditions at 7 weeks of age, but expect that a downshift in housing conditions at any age is worse than keeping pigs in barren conditions throughout, although I would not advocate that this is a reason to refrain pigs from early life enrichment. Hence, it is better to keep the same 
resources after an environmental change or provide a more enriched environment for the pigs after moving to the next stage.

Therefore, it is best to provide pigs with enrichment around weaning as this helps to cope with the weaning transition and avoid the experience of a loss in enrichment for pigs. If pigs do not have bedding substrates or limited living space, pigs could be given routinely rotating objects to explore, especially during the transition periods, as well as some handfuls of organic materials like straw or alfalfa. When new farms are designed, more space allowance for pigs at different life stages, floor types (allowing for bedding substrates), and the locations where enriched materials can be applied need to be considered. These changes are not easy to apply under economically tight production conditions, and an increasing demand on the industry to reduce the environmental impact of the pig production. To be able to improve pig welfare under these conditions, there is an urgent need for an open-minded and obligating collaboration between animal scientists, environmental scientists, companies that develop equipment for the pig industry, animals welfare organisations, as well as other stakeholders from both industry and society.

\subsubsection{Future work}

In this thesis, effects of (early life) environmental enrichment on immune parameters of pigs were demonstrated. It was hypothesized that this was partly through exposure to antigens provided from the enrichment substrates and partly through the effects of environmental enrichment on the mental state and behaviour of the pigs. However, the relative contribution of these factors to the effects found were not studied in this 
thesis. Future work is needed to disentangle the different mechanisms by which (early life) environmental enrichment affects immunity in pigs, and the subsequent consequences for their health and health management procedures such as vaccinations. To study whether affective states affect health of the piglets, effects of affective state need to be separated from the direct antigenic effects of enrichment materials on the (developing) immune system. To this aim, pigs should be kept and tested under similar controlled hygienic conditions while inducing different affective states.

As discussed before, pig behaviour has been widely studied, but reliable and practical measurements of affective states, especially positive ones, in pigs are not well-developed yet. Most tests for assessing affective states in animals are based on studies in humans and need to be adjusted to the specific characteristics of the species under study. The attention bias test and successive contrast test used in this thesis, were, to our knowledge, applied in pigs for the first time and unexpected results were found. It is not clear whether the unexpected results were due to pitfalls in the tests themselves or not. More work is needed to understand pigs' mental experiences, to develop and improve tests to measure their affective state, and to assess validity of other indicators of affective state.

This thesis focused on enrichment of the physical environment, except that in Chapter 3 early life social experiences also varied for enriched and barren housed pigs. More studies are needed to investigate the effects of (early life) social experiences in pigs. Pigs are a highly social species, with social bonds that can persist long after weaning (Newberry and Wood-Gush 1986). It is therefore not surprising that social experiences in early life can have long- 
lasting effects on behaviour (D'Eath 2004; Kanaan et al. 2012), growth and social skills (Canario et al. 2017; Weary et al. 2002). Early life social enrichment, provided by, for instance, comingling or group farrowing systems, might be promising to improve pigs' welfare and resilience with possible impacts on the immune system and health.

More studies on environmental enrichment in sows are needed, as enrichment may not only be important for their own (three pillars of) welfare, but also for that of their offspring. There are relatively few studies investigating whether absence of proper environmental enrichment for sows, likely inducing stress, epigenetically affects her offspring. Early life experiences after birth were found to be important in this thesis, but it has been found that stress experienced by the pregnant mother, via the release of stress hormones that enter the foetal circulation (Migeon et al. 1956) may program the offspring's HPA axis activity in later life (Bosch et al. 2007; Glover et al. 2009) as well. Besides, the effects of maternal stress can be passed on to the offspring through changes in maternal behaviour and maternal care (Champagne et al. 2006; Meaney 2001), and through paternal effects of stress on maternal behaviour (Mashoodh et al. 2012) and offspring development (Mychasiuk et al. 2013). These putative effects of enrichment for sows on their offspring may also interact with enrichment in later life, as studies in other species have revealed that environmental enrichment may provide an effective therapy for those who suffer from the effects of prenatal stress (review in McCreary and Metz 2016). 


\subsection{Conclusions}

To conclude, the results in this thesis support the expectation that environmental enrichment influences the behaviour, affective state and immunity in pigs, which could improve their welfare and health, compared to barren housing conditions. Besides, apart from the current housing conditions, the housing history in early life and the (mis)match between early life and current environments regarding the provision of enrichment, had long-lasting impacts on pigs. The results of this thesis underline the importance of enrichment for pigs, as well as a fit between their early and later life housing conditions. 


\subsection{References}

Abou-Donia MB, Abou-Donia MM, EIMasry EM, Monro JA, Mulder MF (2013) Autoantibodies to nervous system-specific proteins are elevated in sera of flight crew members: biomarkers for nervous system injury Journal of Toxicology and Environmental Health, Part A 76:363-380

Andrejević $S$ et al. (1997) Stress-induced rise in serum anti-brain autoantibody levels in the rat International journal of neuroscience 89:153-164

Asher L, Friel M, Griffin K, Collins LM (2016) Mood and personality interact to determine cognitive biases in pigs Biology letters 12:20160402

Avitsur R, Levy S, Goren N, Grinshpahet R (2015) Early adversity, immunity and infectious disease Stress 18:289-296

Barak $Y$ (2006) The immune system and happiness Autoimmunity reviews 5:523-527

Bayne K, Hurst J, Dexter S (1992) Evaluation of the preference to and behavioral effects of an enriched environment on male rhesus monkeys Laboratory Animal Science 42:38-45

Beattie V, O'connell N, Kilpatrick D, Moss B (2000a) Influence of environmental enrichment on welfare-related behavioural and physiological parameters in growing pigs Animal Science 70:443-450

Beattie V, O'connell N, Moss B (2000b) Influence of environmental enrichment on the behaviour, performance and meat quality of domestic pigs Livestock production science 65:71-79

Berghof T et al. (2019) Selective breeding for high natural antibody level increases resistance to avian pathogenic Escherichia coli (APEC) in chickens Developmental \& Comparative Immunology 93:45-57

Berghof T, Van Der Klein S, Arts J, Parmentier H, Van Der Poel J, Bovenhuis H (2015) Genetic and non-genetic inheritance of natural antibodies binding keyhole limpet hemocyanin in a purebred layer chicken line PLoS One 10:e0131088

Berk M, Wadee A, Kuschke R, O'Neill-Kerr A (1997) Acute phase proteins in major depression Journal of psychosomatic research 43:529-534

Boissy A et al. (2007) Assessment of positive emotions in animals to improve their welfare Physiology \& behavior 92:375-397

Bolhuis JE, Schouten WG, de Leeuw JA, Schrama JW, Wiegant VM (2004) Individual coping characteristics, rearing conditions and behavioural flexibility in pigs Behavioural Brain Research 152:351-360 
Bolhuis JE, Schouten WG, Schrama JW, Wiegant VM (2005a) Behavioural development of pigs with different coping characteristics in barren and substrate-enriched housing conditions Applied Animal Behaviour Science 93:213-228

Bolhuis JE, Schouten WG, Schrama JW, Wiegant VM (2005b) Individual coping characteristics, aggressiveness and fighting strategies in pigs Animal Behaviour 69:1085-1091

Bolhuis JE, Schouten WG, Schrama JW, Wiegant VM (2006) Effects of rearing and housing environment on behaviour and performance of pigs with different coping characteristics Applied Animal Behaviour Science 101:68-85

Bosch OJ, Müsch W, Bredewold R, Slattery DA, Neumann ID (2007) Prenatal stress increases HPA axis activity and impairs maternal care in lactating female offspring: implications for postpartum mood disorder Psychoneuroendocrinology 32:267-278

Camerlink I, Turner SP, Bijma P, Bolhuis JE (2013) Indirect genetic effects and housing conditions in relation to aggressive behaviour in pigs PloS one 8:e65136

Campbell JM, Crenshaw JD, Polo J (2013) The biological stress of early weaned piglets Journal of animal science and biotechnology 4:1

Canario L, Lundeheim N, Bijma P (2017) The early-life environment of a pig shapes the phenotypes of its social partners in adulthood Heredity 118:534

Cao J, Li B, Fang L, Chen H, Xiao S (2012) Pathogenesis of nonsuppurative encephalitis caused by highly pathogenic Porcine reproductive and respiratory syndrome virus Journal of veterinary diagnostic investigation 24:767-771

Casali P, Notkins AL (1989) Probing the human B-cell repertoire with EBV: polyreactive antibodies and CD5+ B lymphocytes Annual review of immunology 7:513-535

Champagne FA, Weaver IC, Diorio J, Dymov S, Szyf M, Meaney MJ (2006) Maternal care associated with methylation of the estrogen receptor$\alpha 1 b$ promoter and estrogen receptor- $\alpha$ expression in the medial preoptic area of female offspring Endocrinology 147:2909-2915

Chen Y et al. (2019) 59 The Genetic Basis of Natural Antibody Titers and Relationships with Disease Resilience in Pigs Journal of Animal Science 97:35-36 
Chrousos GP (1995) The hypothalamic-pituitary-adrenal axis and immunemediated inflammation New England Journal of Medicine 332:13511363

Connor TJ, Leonard BE (1998) Depression, stress and immunological activation: the role of cytokines in depressive disorders Life sciences 62:583-606

Coppens CM, de Boer SF, Koolhaas JM (2010) Coping styles and behavioural flexibility: towards underlying mechanisms Philosophical Transactions of the Royal Society B: Biological Sciences 365:40214028

Cornale P, Macchi E, Miretti S, Renna M, Lussiana C, Perona G, Mimosi A (2015) Effects of stocking density and environmental enrichment on behavior and fecal corticosteroid levels of pigs under commercial farm conditions Journal of Veterinary Behavior: Clinical Applications and Research 10:569-576

Coutellier L, Arnould C, Boissy A, Orgeur P, Prunier A, Veissier I, MeunierSalaün M-C (2007) Pig's responses to repeated social regrouping and relocation during the growing-finishing period Applied Animal Behaviour Science 105:102-114

Coutinho A, Kazatchkine MD, Avrameas S (1995) Natural autoantibodies Current opinion in immunology 7:812-818

Cuervo PF, Beldomenico PM, Sánchez A, Pietrobon E, Valdez SR, Racca AL (2018) Chronic exposure to environmental stressors enhances production of natural and specific antibodies in rats Journal of Experimental Zoology Part A: Ecological and Integrative Physiology 329:536-546

D'Eath RB (2004) Consistency of aggressive temperament in domestic pigs: The effects of social experience and social disruption Aggressive Behavior: Official Journal of the International Society for Research on Aggression 30:435-448

Daskalakis NP, Bagot RC, Parker KJ, Vinkers CH, de Kloet ER (2013) The threehit concept of vulnerability and resilience: toward understanding adaptation to early-life adversity outcome Psychoneuroendocrinology 38:1858-1873

Day J, Burfoot A, Docking C, Whittaker X, Spoolder H, Edwards S (2002a) The effects of prior experience of straw and the level of straw provision on the behaviour of growing pigs Applied Animal Behaviour Science 76:189-202 
Day J, Spoolder H, Burfoot A, Chamberlain H, Edwards S (2002b) The separate and interactive effects of handling and environmental enrichment on the behaviour and welfare of growing pigs Applied Animal Behaviour Science 75:177-192

de Groot J, de Jong IC, Prelle IT, Koolhaas JM (2000) Immunity in barren and enriched housed pigs differing in baseline cortisol concentration Physiology \& behavior 71:217-223

de Jong IC et al. (1998) Effects of strawbedding on physiological responses to stressors and behavior in growing pigs Physiology \& behavior 64:303310

de Jong IC, Prelle IT, van de Burgwal JA, Lambooij E, Korte SM, Blokhuis HJ, Koolhaas JM (2000) Effects of environmental enrichment on behavioral responses to novelty, learning, and memory, and the circadian rhythm in cortisol in growing pigs Physiology \& behavior 68:571-578

de Kloet ER, Sibug RM, Helmerhorst FM, Schmidt M (2005) Stress, genes and the mechanism of programming the brain for later life Neuroscience \& Biobehavioral Reviews 29:271-281

Dobbing J, Sands J (1978) Head circumference, biparietal diameter and brain growth in fetal and postnatal life Early human development 2:81-87

Eid RS, Chaiton JA, Lieblich SE, Bodnar TS, Weinberg J, Galea LA (2019) Early and late effects of maternal experience on hippocampal neurogenesis, microglia, and the circulating cytokine milieu Neurobiology of aging 78:1-17

Foyer P, Wilsson E, Wright D, Jensen P (2013) Early experiences modulate stress coping in a population of German shepherd dogs Applied animal behaviour science 146:79-87

Francis DD, Diorio J, Plotsky PM, Meaney MJ (2002) Environmental enrichment reverses the effects of maternal separation on stress reactivity Journal of Neuroscience 22:7840-7843

Fraser D, Duncan IJ (1998) 'Pleasures','pains' and animal welfare: toward a natural history of affect

Fraser D, Phillips P, Thompson B, Tennessen T (1991) Effect of straw on the behaviour of growing pigs Applied Animal Behaviour Science 30:307318

Geverink N, Heetkamp M, Schouten W, Wiegant V, Schrama J (2004) Backtest type and housing condition of pigs influence energy metabolism Journal of Animal Science 82:1227-1233 
Glaser R, Kiecolt-Glaser JK (2005) Stress-induced immune dysfunction: implications for health Nature Reviews Immunology 5:243

Gley K et al. (2019) Haplotypes of coping behavior associated QTL regions reveal distinct transcript profiles in Amygdala and Hippocampus Behavioural brain research:112038

Glover V, Bergman K, Sarkar P, O'Connor TG (2009) Association between maternal and amniotic fluid cortisol is moderated by maternal anxiety Psychoneuroendocrinology 34:430-435

Gonyou H, Rohde Parfet K, Anderson D, Olson R (1988) Effects of amperozide and azaperone on aggression and productivity of growing-finishing pigs Journal of animal science 66:2856-2864

Haghighi HR et al. (2006) Probiotics stimulate production of natural antibodies in chickens Clin Vaccine Immunol 13:975-980

Hangalapura B, Nieuwland M, de Vries Reilingh G, Heetkamp M, Van den Brand H, Kemp B, Parmentier H (2003) Effects of cold stress on immune responses and body weight of chicken lines divergently selected for antibody responses to sheep red blood cells Poultry Science 82:1692-1700

Hessing M, Coenen G, Vaiman M, Renard C (1995) Individual differences in cell-mediated and humoral immunity in pigs Veterinary Immunology and Immunopathology 45:97-113

Hessing MJ, Hagelsø AM, Schouten WG, Wiepkema PR, Van Beek JA (1994) Individual behavioral and physiological strategies in pigs Physiology \& Behavior 55:39-46

Hestad KA, Tønseth S, Støen CD, Ueland T, Aukrust P (2003) Raised plasma levels of tumor necrosis factor $\alpha$ in patients with depression: normalization during electroconvulsive therapy The journal of ECT 19:183-188

Jansen J, Bolhuis JE, Schouten WG, Spruijt BM, Wiegant VM (2009) Spatial learning in pigs: effects of environmental enrichment and individual characteristics on behaviour and performance Animal cognition 12:303-315

Jones S, Neville V, Higgs L, Paul ES, Dayan P, Robinson ES, Mendl M (2018) Assessing animal affect: an automated and self-initiated judgement bias task based on natural investigative behaviour Scientific reports 8:12400

Kanaan VT, Lay Jr DC, Richert BT, Pajor EA (2012) Increasing the frequency of co-mingling piglets during the lactation period alters the 
development of social behavior before and after weaning Journal of applied animal welfare science 15:163-180

Kanitz E, Tuchscherer M, Otten W, Tuchscherer A, Zebunke M, Puppe B (2019) Coping style of pigs is associated with different behavioral, neurobiological and immune responses to stressful challenges Frontiers in behavioral neuroscience 13:173

Kanitz E, Tuchscherer M, Puppe B, Tuchscherer A, Stabenow B (2004) Consequences of repeated early isolation in domestic piglets (Sus scrofa) on their behavioural, neuroendocrine, and immunological responses Brain, Behavior, and Immunity 18:35-45

Kaufman J, Plotsky PM, Nemeroff CB, Charney DS (2000) Effects of early adverse experiences on brain structure and function: clinical implications Biological psychiatry 48:778-790

Kiley M (1972) The vocalizations of ungulates, their causation and function Zeitschrift für Tierpsychologie 31:171-222

Kim SJ, Gershov D, Ma X, Brot N, Elkon KB (2002) I-PLA2 activation during apoptosis promotes the exposure of membrane lysophosphatidylcholine leading to binding by natural immunoglobulin $M$ antibodies and complement activation Journal of Experimental Medicine 196:655-665

Koh KB (1998) Emotion and immunity Journal of Psychosomatic Research 45:107-115

Koh S, Chung H, Xia H, Mahadevia A, Song Y (2005) Environmental enrichment reverses the impaired exploratory behavior and altered gene expression induced by early-life seizures Journal of child neurology 20:796-802

Koolhaas J et al. (1999) Coping styles in animals: current status in behavior and stress-physiology Neuroscience \& Biobehavioral Reviews 23:925-935

Koolhaas JM, De Boer SF, Buwalda B, Van Reenen K (2007) Individual variation in coping with stress: a multidimensional approach of ultimate and proximate mechanisms Brain, behavior and evolution 70:218-226

Krause A, Puppe B, Langbein J (2017) Coping style modifies general and affective autonomic reactions of domestic pigs in different behavioral contexts Frontiers in behavioral neuroscience 11:103

Kreuk LS et al. (2019) B cell receptor and Toll-like receptor signaling coordinate to control distinct B-1 responses to both self and the microbiota eLife 8 
Lacey RE, Kumari M, McMunn A (2013) Parental separation in childhood and adult inflammation: the importance of material and psychosocial pathways Psychoneuroendocrinology 38:2476-2484

Lammers A, Klomp ME, Nieuwland MG, Savelkoul HF, Parmentier HK (2004) Adoptive transfer of natural antibodies to non-immunized chickens affects subsequent antigen-specific humoral and cellular immune responses Developmental \& Comparative Immunology 28:51-60

Latham N, Mason G (2010) Frustration and perseveration in stereotypic captive animals: is a taste of enrichment worse than none at all? Behavioural Brain Research 211:96-104

Leliveld LM, Düpjan S, Tuchscherer A, Puppe B (2016) Behavioural and physiological measures indicate subtle variations in the emotional valence of young pigs Physiology \& behavior 157:116-124

Leonard B, Song C (1999) Stress, depression, and the role of cytokines. In: Cytokines, stress, and depression. Springer, pp 251-265

Lewis CR, Olive MF (2014) Early life stress interactions with the epigenome: potential mechanisms driving vulnerability towards psychiatric illness Behavioural pharmacology 25:341

Lutz HU, Binder CJ, Kaveri S (2009) Naturally occurring auto-antibodies in homeostasis and disease Trends in immunology 30:43-51

Maes M (1999) Major depression and activation of the inflammatory response system. In: Cytokines, stress, and depression. Springer, pp 25-46

Malmkvist J, Hansen SW (2002) Generalization of fear in farm mink, Mustela vison, genetically selected for behaviour towards humans Animal Behaviour 64:487-501

Manson JJ, Mauri C, Ehrenstein MR Natural serum IgM maintains immunological homeostasis and prevents autoimmunity. In: Springer seminars in immunopathology, 2005. vol 4. Springer, pp 425-432

Mashoodh R, Franks B, Curley JP, Champagne FA (2012) Paternal social enrichment effects on maternal behavior and offspring growth Proceedings of the National Academy of Sciences 109:17232-17238

Matsunaga $M$ et al. (2013) Brain-immune interaction accompanying odorevoked autobiographic memory PLoS One 8:e72523

McCreary JK, Metz GA (2016) Environmental enrichment as an intervention for adverse health outcomes of prenatal stress Environmental epigenetics 2 
Meaney MJ (2001) Maternal care, gene expression, and the transmission of individual differences in stress reactivity across generations Annual review of neuroscience 24:1161-1192

Meaney MJ, Aitken DH, Viau V, Sharma S, Sarrieau A (1989) Neonatal handling alters adrenocortical negative feedback sensitivity and hippocampal type II glucocorticoid receptor binding in the rat Neuroendocrinology 50:597-604

Mendl M, Erhard HW, Haskell M, Wemelsfelder F, Lawrence AB (1997) Experience in substrate-enriched and substrate-impoverished environments affects behaviour of pigs in a T-maze task Behaviour:643-659

Migeon CJ, Prystowsky H, Grumbach MM, Byron MC (1956) Placental passage of 17-hydroxycorticosteroids: comparison of the levels in maternal and fetal plasma and effect of ACTH and hydrocortisone administration The Journal of clinical investigation 35:488-493

Moore A, Gonyou H, Stookey J, McLaren D (1994) Effect of group composition and pen size on behavior, productivity and immune response of growing pigs Applied Animal Behaviour Science 40:13-30

Mulder IE et al. (2009) Environmentally-acquired bacteria influence microbial diversity and natural innate immune responses at gut surfaces BMC biology $7: 1$

Munsterhjelm C, Valros A, Heinonen M, Hälli O, Siljander-Rasi H, Peltoniemi O (2010) Environmental enrichment in early life affects cortisol patterns in growing pigs Animal 4:242-249

Mychasiuk R, Harker A, Inytskyy S, Gibb R (2013) Paternal stress prior to conception alters DNA methylation and behaviour of developing rat offspring Neuroscience 241:100-105

Newberry R, Wood-Gush D (1986) Social relationships of piglets in a seminatural environment Animal Behaviour 34:1311-1318

Ochsenbein AF, Zinkernagel RM (2000) Natural antibodies and complement link innate and acquired immunity Immunology today 21:624-630

Oostindjer M, van den Brand H, Kemp B, Bolhuis JE (2011) Effects of environmental enrichment and loose housing of lactating sows on piglet behaviour before and after weaning Applied Animal Behaviour Science 134:31-41

Oster M, Scheel M, Muráni E, Ponsuksili S, Zebunke M, Puppe B, Wimmers K (2015) The fight-or-flight response is associated with PBMC expression profiles related to immune defence and recovery in swine PloS one 10:e0120153 
Parmentier HK, Lammers A, Hoekman JJ, Reilingh GDV, Zaanen IT, Savelkoul HF (2004) Different levels of natural antibodies in chickens divergently selected for specific antibody responses Developmental \& Comparative Immunology 28:39-49

Pecoraro NC, Timberlake WD, Tinsley M (1999) Incentive downshifts evoke search repertoires in rats Journal of Experimental Psychology: Animal Behavior Processes 25:153

Petersen V, Simonsen HB, Lawson LG (1995) The effect of environmental stimulation on the development of behaviour in pigs Applied Animal Behaviour Science 45:215-224

Prossin A, Koch A, Campbell P, Barichello T, Zalcman S, Zubieta J (2016) Acute experimental changes in mood state regulate immune function in relation to central opioid neurotransmission: a model of human CNSperipheral inflammatory interaction Molecular psychiatry 21:243

Quartier P, Potter PK, Ehrenstein MR, Walport MJ, Botto M (2005) Predominant role of IgM-dependent activation of the classical pathway in the clearance of dying cells by murine bone marrowderived macrophages in vitro European journal of immunology 35:252-260

Raison CL et al. (2009) Activation of central nervous system inflammatory pathways by interferon-alpha: relationship to monoamines and depression Biological psychiatry 65:296-303

Reimert I, Bolhuis JE, Kemp B, Rodenburg TB (2015) Emotions on the loose: emotional contagion and the role of oxytocin in pigs Animal cognition 18:517-532

Reimert I, Rodenburg TB, Ursinus WW, Kemp B, Bolhuis JE (2014a) Responses to novel situations of female and castrated male pigs with divergent social breeding values and different backtest classifications in barren and straw-enriched housing Applied animal behaviour science 151:24-35

Reimert I, Rodenburg TB, Ursinus WW, Kemp B, Bolhuis JE (2014b) Selection based on indirect genetic effects for growth, environmental enrichment and coping style affect the immune status of pigs PloS one 9:e108700

Ruis MA, te Brake JH, Engel B, Buist WG, Blokhuis HJ, Koolhaas JM (2001) Adaptation to social isolation: acute and long-term stress responses of growing gilts with different coping characteristics Physiology \& behavior 73:541-551 
Schmeer KK, Ford JL, Browning CR (2019) Early childhood family instability and immune system dysregulation in adolescence Psychoneuroendocrinology 102:189-195

Schokker D et al. (2014) Early-life environmental variation affects intestinal microbiota and immune development in new-born piglets PLoS One 9:e100040

Schrama J, Schouten J, Swinkels J, Gentry J, de Vries Reilingh G, Parmentier H (1997) Effect of hemoglobin status on humoral immune response of weanling pigs differing in coping styles Journal of Animal Science 75:2588-2596

Spinka M, Newberry RC, Bekoff M (2001) Mammalian play: training for the unexpected The quarterly review of biology 76:141-168

Star L, Frankena K, Kemp B, Nieuwland M, Parmentier H (2007) Natural humoral immune competence and survival in layers Poultry science 86:1090-1099

Stookey JM, Gonyou HW (1994) The effects of regrouping on behavioral and production parameters in finishing swine Journal of animal science 72:2804-2811

Sun Y, Parmentier H, Frankena K, Van der Poel J (2011) Natural antibody isotypes as predictors of survival in laying hens Poultry science 90:2263-2274

Tan S, Shackleton D, Beames R (1991) The effect of mixing unfamiliar individuals on the growth and production of finishing pigs Animal Science 52:201-206

Telkänranta H, Edwards SA (2018) Lifetime consequences of the early physical and social environment of piglets. In: Advances in Pig Welfare. Elsevier, pp 101-136

Thanawongnuwech R, Halbur PG, Andrews JJ (1997) Immunohistochemical detection of porcine reproductive and respiratory syndrome virus antigen in neurovascular lesions Journal of veterinary diagnostic investigation 9:334-337

Tönepöhl B, Appel AK, Welp S, Voß B, von Borstel UK, Gauly M (2012) Effect of marginal environmental and social enrichment during rearing on pigs' reactions to novelty, conspecifics and handling Applied animal behaviour science 140:137-145

Tuyttens FAM (2005) The importance of straw for pig and cattle welfare: a review Applied animal behaviour science 92:261-282 
Ursinus WW et al. (2013) Relations between peripheral and brain serotonin measures and behavioural responses in a novelty test in pigs Physiology \& behavior 118:88-96

Ursinus WW, Van Reenen CG, Reimert I, Bolhuis JE (2014) Tail biting in pigs: blood serotonin and fearfulness as pieces of the puzzle? PloS one 9:e107040

van de Weerd HA, Day JE (2009) A review of environmental enrichment for pigs housed in intensive housing systems Applied Animal Behaviour Science 116:1-20

van Dixhoorn ID et al. (2016) Enriched housing reduces disease susceptibility to co-infection with porcine reproductive and respiratory virus (PRRSV) and Actinobacillus pleuropneumoniae (A. pleuropneumoniae) in young pigs PloS one 11:e0161832

Van Knegsel A, de Vries Reilingh G, Meulenberg S, Van den Brand H, Dijkstra J, Kemp B, Parmentier H (2007) Natural antibodies related to energy balance in early lactation dairy cows Journal of Dairy Science 90:5490-5498

Vermeer H, De Greef K, Houwers H (2014) Space allowance and pen size affect welfare indicators and performance of growing pigs under Comfort Class conditions Livestock Science 159:79-86

Weary DM, Pajor EA, Bonenfant M, Fraser D, Kramer DL (2002) Alternative housing for sows and litters.: Part 4. Effects of sow-controlled housing combined with a communal piglet area on pre-and postweaning behaviour and performance Applied Animal Behaviour Science 76:279-290

Wiegmann DD, Wiegmann DA, Waldron FA (2003) Effects of a reward downshift on the consummatory behavior and flower choices of bumblebee foragers Physiology \& behavior 79:561-566

Wondmeneh E, Van Arendonk J, Van der Waaij E, Ducro B, Parmentier H (2015) High natural antibody titers of indigenous chickens are related with increased hazard in confinement Poultry science 94:1493-1498 


\section{Appendix:}

\section{Novel environment and novel object test}

In the novel environment and novel object test (NET-NOT), 128 pigs (4 pigs/pen, from 32 pens), balanced for sex and coping style, were placed in a novel test arena, away from their home pens, for $10 \mathrm{~min}$. Pigs were part of the pigs from the study described in Chapter 4 and 7 and details on their housing conditions can be found in these chapters. The test was carried out at 10 weeks of age and was distributed over 4 days per batch, balanced for housing treatments. After $5 \mathrm{~min}$ in the arena, which was a novel environment for the pigs, a novel object was introduced. The novel object (a metal bucket) was dropped from the ceiling slowly until it touched the floor and made a noise to alert the pig. During the second $5 \mathrm{~min}$ of the test, the novel object was left in the arena. During the test, the behaviours and vocalisations, as shown in the ethogram in Table 1, were recorded using focal sampling and continuous recording. States were expressed as percentages of time, and events as frequencies. The latencies to approach (see Table 1) and to touch the bucket (in sec) were also scored. Effects of early life housing, current housing, coping style, their interactions, batch, and sex on behaviours in the NET-NOT were analysed separately for the 5 min before and after the drop of the novel object, using linear mixed models that included a random effect of pen within early life and current housing, and batch.

To measure cortisol concentrations, saliva samples were collected from each pig in the home pen just before the pig was transferred to the test arena $(t=0)$, and 15, 30 and 60 min after the start of the test by allowing the pigs to chew on two cotton buds in the home pen. All pigs were habituated to chew on 
cotton buds in the week before the NET-NOT. Cortisol concentration $(\mathrm{ng} / \mathrm{ml})$ were measured in duplicate using a cortisol saliva ELISA kit (IBL International, Switzerland). Effects of the factors as mentioned above, to which sampling time was added, were analysed in a repeated mixed model, with random effects of pen and pig to account for repeated measurements at pen and pig level. The results are presented in Boxes 1 and 2 of Chapter 8.

Table 1: Ethogram of behaviours scored in novel environment - novel object test (Reimert et al. 2014a; Ursinus et al. 2013).

\begin{tabular}{|c|c|}
\hline Behaviour & Definition \\
\hline \multicolumn{2}{|c|}{ Locomotion class (state) } \\
\hline Walking & $\begin{array}{l}\text { Walking without performing any other described behaviour. All } 4 \text { legs move, or the pig turns } \\
\text { around at the same spot without moving all } 4 \text { legs. }\end{array}$ \\
\hline Standing alert & $\begin{array}{l}\text { Standing motionless with head fixed (up or down) and ears upright, focused on a noise, wall, } \\
\text { person, object etc. }\end{array}$ \\
\hline Standing & Standing with four paws on the floor without performing any other described behaviour. \\
\hline \multicolumn{2}{|c|}{ Behaviour class (state) } \\
\hline Exploring & $\begin{array}{l}\text { Exploring the floor and/or the walls of the arena by sniffing, nosing, rooting, or licking it with the } \\
\text { rooting disc. }\end{array}$ \\
\hline $\begin{array}{l}\text { Draw back from } \\
\text { bucket }\end{array}$ & The pig draws back from the bucket. \\
\hline Explore bucket & $\begin{array}{l}\text { Nosing, sniffing, rooting or licking the metal bucket; trying to chew on the metal bucket and rope } \\
\text { by touching it with open mouth and making biting movements. }\end{array}$ \\
\hline Other & The pig is performing not any of the abovementioned behaviours in the behaviour class. \\
\hline \multicolumn{2}{|c|}{$\underline{\text { Vocalisations (event) }}$} \\
\hline Grunts & $\begin{array}{l}\text { Short and long grunts combined, low tone of less than half a second or of more than half a second } \\
\text { (one tone). }\end{array}$ \\
\hline $\begin{array}{l}\text { High-pitched } \\
\text { vocalisations }\end{array}$ & High tones, combining squeals, screams and grunt-squeals. \\
\hline \multicolumn{2}{|c|}{ Other behaviours (event) } \\
\hline Defecating & Defecating. \\
\hline Urinating & Urinating. \\
\hline
\end{tabular}




\section{Factor analysis and correlation analysis}

To explore potential relationships between immune variables with behavioural, affective state (fear-related behaviours in the novel environment - novel object test (NET-NOT)), and growth data in this thesis, Pearson correlations were used. In total, 64 pigs from 32 pens in 2 batches (balanced for coping style, sex and (early life) housing treatment), were immunized. Due to death of pigs (see Chapter 7 for details) and missing values related to technical problems, correlations were done on 56 pigs in total.

To reduce the number of variables and thereby make the results less complex, a factor analysis (FA) was conducted on immune data (Chapter 7), behaviours in the home pen (Chapter 4) and behaviours in the NET-NOT (Box 1 of Chapter 8 and section 1 of this Appendix) separately. Prior to FA, correlations between variables within each test were calculated, and the variables which were highly correlated with other variables $(r>0.80)$, were combined (grunts and high-pitched vocalisations in NET-NOT) or excluded (latency to approach the novel object was excluded as it highly correlated with latency to touch the object). Time spent on oral manipulation of pen mates was arcsin square root transformed, and the relative frequency of the monocytes after immunization and latency to touch the bucket in the NET-NOT were log transformed to normalize the variables. As early and later life housing, and their interaction, had profound effects on behaviours in the home pen (Chapter 7) and in the NET-NOT (Chapter 8, Box 1), as well as on immune variables (Chapter 7) and growth (Chapter 4), a general linear model was run, with early and later life housing as factors. The residuals obtained were used 
for FA and correlation analyses. Residuals of variables were entered into a FA with orthogonal varimax rotation and factors with eigenvalue above one were retained. Subsequently, factor scores of each pig were used in correlations.

\subsection{Factor analysis for antibodies and leukocyte subpopulations}

Natural IgM and IgG antibodies binding KLH before immunization, the area under the curve during primary and secondary immunizations for KLH-IgM and KLH-IgG, the relative frequencies of leukocytes subpopulations measured at 74 days (before immunizations) and 130 days (after immunizations) of age were included in the FA for immune parameters. The FA resulted in eight factors (Table 8.3); therefore, the pigs' scores of these eight factors were further included in the Pearson correlation analysis.

\subsection{Factor analysis for behaviours in the home pen}

Behaviours used are described in the ethogram in Chapter 4. The average percentages of time spent on the behaviours over the 5 observation days after the housing switch (at 7 weeks of age) were analysed. Residuals of inactivity, aggression, manipulation, social behaviour, play behaviour, exploring and chewing (combination of chewing pen, air or substrates) were included in FA for home pen behaviours. Because the FA for home pen behaviours only resulted in one factor, the individual variables (residuals) were included in the Pearson correlation analysis.

2.3 Factor analysis for behaviours in the novel environment - novel object test 
The variables walking, standing alert, standing, exploring, explore bucket, and vocalisations (combination of grunts and high-pitched vocalisations) were used, as well as the latency to touch the bucket. Drawback from the bucket was excluded, because it could not be normalized. The FA resulted in four factors (Table 8.4); therefore, the pigs' scores of these four factors were included in the Pearson correlation analysis.

\subsection{Correlations}

The correlations between the factor scores of the eight factors containing the immune variables with the residuals of the behaviours in the home pen, factor scores of the NET-NOT, and the growth from day 46 to 50 (G46-50, from before until 3 days after the housing switch), from day 50 to 74 (G5074), from day 74 to 109 (G74-109, primary immunization period), from day 109 to 130 (G109-130, secondary immunization period), and from day 46 to 130 (G46-130, complete period from the housing switch until the end of the experiment) were calculated by Pearson correlations. The correlations are presented in Chapter 8. Correlations between immune factors and the area under the curve of cortisol concentrations in the NET-NOT were not significant. 



\section{Summary}

Welfare and health issues in pigs have become increasingly important to the public and in science. Worldwide, most commercial pigs are housed in rather barren, stimulus-poor housing conditions. These barren conditions limit the expression of their natural species-specific behaviours, leading to the development of damaging behaviours, such as tail biting and ear biting. Besides, barren housing can also induce (chronic) stress in pigs, as reflected in physiological changes, and this could potentially impact their immune competence and health. Environmental enrichment, opposite to barren housing, allows pigs to perform more of their natural behaviours. Especially enrichment with rooting substrates facilitates the display of exploratory behaviours and reduces tail biting and other damaging behaviours, thereby improving the welfare of pigs. The contemporary concept of animal welfare not only considers the animals' ability to live reasonably natural lives and to express their natural behaviour, but also includes experiencing minimal negative affective states and exhibiting good physical health and biological functioning. The impact of environmental enrichment on affective state and health in pigs, however, is less well-studied, and therefore, more research is needed.

Apart from the presence of environmental enrichment per se, the effects of environmental enrichment may derive from developmental changes caused by early life housing experiences. Moreover, given the fact that most pigs are relocated at least once during their lives, it is vital to consider the impact of a change in environment, potentially causing a mismatch between early life and current housing conditions. Research on this topic in pigs is, however, 
scarce. Therefore, the main aim of this thesis was to investigate whether and how early life and current environmental enrichment affect behaviour, affective state, and immunity (which may influence health) in pigs. The coping style of the pigs was also taken into account in this thesis, as it has been suggested that the impact of environment and a change in environment on behaviour and affective state may differ for pigs with diverging coping styles. Besides, the coping style of pigs may explain part of the individual variation in immunity.

Previously, barren housed and enriched housed pigs were found to differ in levels of natural antibody (NAb) binding to keyhole limpet hemocyanin (KLH). $N A b$ are defined as immunoglobulins without exogenous antigenic stimulation, and are important as first line of defence. A large part of NAb are directed to self-antigens, and are referred to as natural autoantibodies. Therefore, a study was conducted to investigate the chronic effects of environmental enrichment vs. barren housing conditions on natural (auto)antibodies (NA(A)b binding danger (phosphorylcholine, PC) and neural protein (Myelin basic protein, MBP) antigens in relatively healthy pigs (Chapter 2). In this study, 480 pigs in 80 pens were housed in either barren or straw-enriched pens from 4 (weaning) to 23 weeks of age. A $24 \mathrm{~h}$ regrouping test was conducted at 9 weeks of age as an acute stressor. Blood samples were taken from pigs before (week 8), and 3 days after the regrouping test (week 9), and at 22 weeks of age. $\operatorname{lgM}$, IgG, and IgA antibody titers binding to Phosphorycholine-conjugated bovine serum albumin (PC$\mathrm{BSA}$ ) and IgM and IgG titers binding to MBP in serum were measured using an indirect Enzyme-linked immunosorbent assay (ELISA). Enriched housed pigs had higher levels of MBP-IgM, and tended to have higher levels of MBP- 
IgG and PC-BSA-IgA than barren housed pigs. Housing effects did not significantly influence the changes in $N A(A) b$ levels due to the regrouping test, except that PC-BSA IgA tended to increase more in enriched housed pigs, and no effect of coping style was found. Effects of housing on MBP autoantibody (mainly IgM) levels and to a lesser extent on PC antibody levels tentatively suggest that environmental conditions affect levels of autoantibodies binding (neural) antigens as a homeostatic response, which could have consequences for pigs' health and welfare.

We found that housing conditions (enriched vs. barren) can influence $N A(A) b$ levels in relatively healthy pigs, which may affect immune protection, and influence the onset and outcome of infection. We therefore studied the effects of environmental enrichment vs. barren housing conditions on levels of $N A(A) b$ in pigs co-infected with porcine reproductive and respiratory syndrome virus (PRRSV) and Actinobacillus pleuropneumoniae (A. pleuropneumoniae) in Chapter 3. In this study, 56 pigs were housed in either barren or enriched (with double living space and substrates) pens from birth to 54 days of age. They were transported and relocated at 39 days of age and infected with PRRSV at 44 days of age, and with $A$. pleuropneumoniae 8 days later. Blood samples were taken at 38, 44, 46, 48, 52, 53 and 54 days of age, and natural IgM and IgG antibody titers binding to $K L H, M B P$, and PC-BSA were measured by a two-step indirect ELISA. Housing significantly affected the overall levels of $N A(A) b$ binding $K L H, M B P$ and PC-BSA, sometimes depending on sampling day. Before infection barren housed pigs had significantly higher levels of $N A(A) b$ than enriched housed pigs, except for KLH-IgM and PC-BSA-IgG, and this is contrast with what we found in Chapter 2. The discrepancy could be due to the differences in starting age, the period 
of application and the type of the enrichment. Infection only affected the IgM binding KLH, MBP, and PC-BSA, but not the IgG isotype. Besides, the changes of antibody levels from day 38 to 44 (before and 5 days after transport and relocation) were for most $\mathrm{NA}(\mathrm{A}) \mathrm{b}$ more pronounced in the enriched housed pigs than in the barren housed pigs. Moreover, changes in MBP-IgM and PCBSA-IgM following infection were also different for enriched and barren housed pigs. Enriched pigs, which displayed a faster clearance of viral RNA, also showed a stronger increase in MBP-IgM levels. Remarkably, within barren housed pigs, a higher increase in MBP-IgM following infection related to a faster reduction of viral RNA in serum, which could suggest that MBPIgM has a beneficial effect on the course of PRRSV infection. We also found a lower viral load in pigs with higher levels of MBP-IgG before infection (Chapter 8). Therefore, it is speculated that MBP-NA(A)b levels, both the circulating levels of $\lg G$ at the onset of infection, and the $\operatorname{lgM}$ antibodies that increased following infection, are related to the response to PRRSV infection in those pigs.

In brief, in Chapter 2 and 3 we found that environmental enrichment per se has impact on immunity in pigs, by affecting NA(A)b levels and their changes in response to challenges and infection. Thereafter we aimed to further investigate the effects of early life environmental enrichment and a (mis)match in early life and current housing on behaviour, affective state, and immunity in pigs. To this aim, pigs from 30 litters were studied. Pigs were housed in either barren or enriched (with more space, substrates, and extra enrichment) pens from birth. Pigs were exposed to a backtest at two weeks of age to assess their coping styles, and classified as reactive (LR) or proactive (HR) based on this test. At 28 days of age, pigs were weaned, and in total 192 
pigs (96 per batch) were selected and regrouped in 32 new pens containing 6 non-littermate pigs each. All pigs were equally regrouped by taking sex (3 males and 3 females), coping style ( 3 HR and 3 LR) and body weight into account. Housing treatment (barren vs. enriched) for each pig was kept the same as before weaning. At 47 days of age, half of the groups of pigs experienced a switch in housing type, and the other half did not. Thus, after the switch, there were four housing treatment groups: barren-barren (B1B2), barren-enriched (B1E2), enriched-enriched (E1E2) and enriched-barren (E1B2), $n=8$ pens per group.

First, in Chapter 4, we studied the (long-term) effects of early life and current environmental enrichment on behaviour and growth in pigs with diverging coping styles. Behaviour of individual pigs before (at day 20 and 21) and after weaning (at day 45, 49, 54, 60, 78, and 125) was scored by live instantaneous scan sampling. Body weight and feed intake of the pigs were measured. Environmental enrichment increased time spent on exploring, chewing and play, and decreased damaging behaviours and pen-directed exploring and chewing. Behaviour of pigs that switched from barren to enriched pens or vice versa reflected not only their current environment, but also the early life housing conditions. Effects of early life and current enrichment on most behaviours were in opposite direction. The effects of (lack of) enrichment after the switch were therefore more pronounced in pigs that had experienced a different early life condition. For instance, pigs experiencing an upgrade from barren to enriched pens (B1E2) seemed to 'catch up' by showing more exploration and play. Conversely, pigs exposed to a downgrade (E1B2) displayed more oral manipulation of pen mates than B1B2 pigs, which particularly held for LR pigs. Effects of early life and current 
housing on several other behaviours depended on coping style as well. Pigs housed in enriched conditions appeared better able to cope with weaning, as they gained more weight and had higher feed intake post-weaning. Barren housed pigs had a lower body weight than enriched pigs just before the switch, after which growth was mainly determined by actual housing, with enriched kept pigs having a higher feed intake and final body weight. Thus, not only current housing conditions, but also a (mis)match with the early life environment may affect behaviour and growth of pigs.

Based on the effects of early life and current environmental enrichment on behaviour found in Chapter 4, barren housed pigs were expected to have a more negative affective state than enriched pigs, and even more so if they originated from an enriched early life environment, i.e. experienced a downgrade in housing conditions. To investigate whether housing experiences in early life, and a (mis)match with current housing conditions, would have long-term effects on affective state, pigs were exposed to a number of tests. In a novel object test (NOT) at 43 days of age before the housing switch, pigs housed in an enriched environment were faster to approach and touch the novel object than pigs housed in a barren environment, which may suggest that enrichment reduced fearfulness in pigs.

Furthermore, a novel environment and novel object test (NET-NOT, in Box 1 and 2, and Appendix), indicating fearfulness, was carried out at 10 weeks of age. After $5 \mathrm{~min}$ in a novel environment, a novel object was introduced for another $5 \mathrm{~min}$. During the test, behaviour and vocalisations were recorded. Saliva samples were taken before they left home pen to the test arena $(t=0)$, 
and 15, 30, and $60 \mathrm{~min}$ after the start of the test, to measure cortisol concentrations. Before the drop of the novel object, E2 pigs showed more walking and standing alert, than B2 pigs, and they grunted more often. Early life enrichment also increased standing alert and grunting, and E1E2 pigs showed more high-pitched vocalisations than other groups. After the drop of the novel object, B1B2 pigs showed less standing alert than E1B2 and B1E2, with E1E2 pigs in between. B1B2 pigs showed more exploration, but this held for B1B2-LR pigs only, and B1B2-LR pigs also showed the longest latency to touch the novel object. E2 pigs had a higher frequency of grunts and highpitched vocalisations than B2 pigs after the drop of the novel object, and spent less time exploring the novel object. The average cortisol concentration over four sampling times was affected by current housing, but not early life housing, and B2 pigs had higher basal cortisol levels than E2 pigs. More standing alert and more vocalisations in enriched pigs seems to show that enriched pigs were more fearful in NET-NOT, however, they did not show a longer latency to touch the novel object, nor a higher cortisol response to the test. It could be that their behaviour is partly socially motivated, which means that their higher levels of vocalisation and standing alert reflect attempts to seek contact with their pen mates. In conclusion, both early life and current environmental enrichment influenced the behaviour in NET-NOT, sometimes dependent on coping style.

Affective state can influence cognitive process, such as memory, judgement, decision-making, and attention. Negative affective states, such as anxiety or depression, can result in an attention bias towards a potential threat and enhance the sensitivity to reward loss. In Chapter 5, to assess attention bias in pigs, a $180 \mathrm{sec}$ attention bias test (ABT) was carried out with 128 pigs, 
when they were around 76 days of age. Four pigs per pen were selected from $B 1 B 2, B 1 E 2, E 1 E 2$, and E1B2 housing conditions, with an equal number of each sex and coping style. Half of the pigs were exposed to a $10 \mathrm{sec}$ (nonsocial, unfamiliar) threat as experimental group, and the other half were not and served as control group. Behaviours and vocalisations of the pigs and attention towards the threat were scored continuously using behaviour sampling. Pigs exposed to the threat paid more attention to the location of the threat, were more vigilant, showed less eating, more walking and were more likely to utter high-pitched vocalisations than the non-threat control pigs. During the presence of the threat, HR pigs from current enriched pens (E2-HR, i.e. B1E2 + E1E2) showed more vigilance but less exploration than others. After threat removal, no effect was found on time spent on paying attention to the threat, vigilance and eating, but E2-HR pigs paid attention towards the threat more frequently, were more likely to utter high-pitched vocalisations and walked more compared to (part of) the other groups, suggesting the most negative affective state in these pigs. Thus, this study suggested that current housing, but not early life housing, affected behaviour in a coping-style-dependent manner in this ABT. Housing effects were opposite to expectation. This might be due to the short-term effect of the relative contrast between the home pens of the pigs and the test room, overruling putative long-term effects of environmental conditions on attention bias.

To investigate whether early life and current environmental enrichment influences the sensitivity to reward loss in pigs, a successive negative contrast (SNC) test was applied from 84 to 120 days of age (Chapter 6). Two female pigs per pen (one HR and one LR), in total 64 pigs, were selected from 32 
pens. One pig per pen was trained to run for a large reward and one for a small reward, and reward loss was introduced for pigs receiving large reward after 11 days (reward downshift), i.e. from then onwards, they received small reward for another 11 days. Pigs housed in barren conditions throughout life (B1B2) generally had a lower probability and higher latency to get the reward than other pigs. HR pigs ran overall slower than LR pigs, and after the reward shift, all pigs ran slower than before. Nevertheless, reward downshift increased the latency and reduced the probability to get to the reward, but only in pigs exposed to barren conditions in early life, which thus were more sensitive to reward loss than pigs from enriched early life housing. In conclusion, barren rearing and housing seemed to reduce the motivation to get to the reward, and barren early life housing experiences had a long-term effect the on sensitivity to reward loss. No clear evidence for an effect of current housing, nor for an effect of a change in housing (from barren to enriched or vice versa) on reward loss sensitivity was found, however.

The outcomes of the tests of affective state do not confirm the results on behaviour, growth, and basal cortisol level, which suggest a poorer welfare in barren housed pigs, and, when looking at behaviour, more so in animals that had experienced enrichment in early life and consequently experienced a downgrade in housing. The tests used may not have been optimal for different reasons and/or need further adjustment. Therefore, more research is needed on the optimal method to assess the long-term effects of rearing and housing on pigs' affective state.

To assess the effects of early life and current housing conditions on innate and adaptive immune competence (Chapter 7), two pigs per pen (balanced 
for sex and coping style within treatment), $n=16$ per treatment, i.e. in total 64 pigs were primary immunized with keyhole limpet hemocyaninconjugated trinitrophenyl (KLH-TNP) at 74 days of age, and secondary immunized with the same dose of antigen at 109 days of age. Blood samples were collected on $74,81,88,95$, and 109 days of age (i.e. 0, 1, 2, 3 and 5 weeks after first immunization), and on 116, 123, and 130 days of age (i.e. 1, 2 , and 3 weeks after second immunization) and IgM and IgG antibody responses were measured. Leukocyte subpopulations were measured on day 74 and 130 . Time course of the antibody responses was not affected by housing. Early life enrichment increased the IgG response to KLH, particularly the primary one. At day 74 the relative frequency of lymphocytes, DC and SLA-II expression on monocytes were higher in E1 pigs, whereas the percentage of granulocytes tended to be lower in E1 pigs at day 74. Early life enrichment increased the SLA-II expression on monocytes, the granulocyte to lymphocyte ratio, and tended to increase the percentage of granulocytes, but tended to decrease the percentage of monocytes at day 130. Later life enrichment reduced percentages of $\mathrm{CD} 4+\mathrm{CD} 8 \alpha^{+} \mathrm{T}$ cells before and after immunization and the SLA-II expression on monocytes at day 74, the percentage of granulocytes and the granulocyte to lymphocyte ratio at day 130. KLH-IgM responses (both primary and secondary) were affected by the interaction between early and later life housing. IgM titers were higher for B1B2 than for E1E2, with the switched animals (B1E2 and E1B2) moving towards the titers of the animals kept in their later life environment from birth onwards. At day 130 the percentage of gamma delta T cells, CD $8 \alpha^{+}$ cytotoxic T cells and DC were not different between B1B2 and E1E2 pigs, but there was a clear impact of the switch in housing conditions on gamma delta 
T cells, particularly for B1E2 pigs. We also found effects of coping style and sex on some immune parameters. In conclusion, both early life and later life enrichment, and, notably a switch in housing conditions influenced specific antibodies and leukocyte subpopulations in pigs and regarding the relative frequencies of leukocyte subsets, the immune system seems to respond to a change in housing condition, in either direction, rather than to the long-term effects of housing per se. This study implies that the early life history of animals and the (mis)match with their current environment could thus be of major importance for their immune system.

In this thesis, we hypothesized that the effects of environmental enrichment on immune competence and responsiveness could be mediated partly through exposure to antigens provided from the enrichment substrates and partly through the effects of environmental enrichment on (chronic) stress, mental state and behaviour. To further explore the relationships between immunity and behaviour and/or affective states, correlations between behaviours of pigs in their home pen and in the NET-NOT and immune parameters (NAb, specific antibodies and leukocyte subpopulations) were assessed, and growth in different periods was also included. A factor analysis was conducted to reduce the number of immune variables, resulting in eight factors. The first factor, with mainly high loadings for T-cell types, was related to play behaviour in the home pen, which may reflect a positive affective state. The second factor, that had a high positive loading for lymphocytes and dendritic cells on the last sampling day and a high negative loading for granulocytes, negatively related to growth of the pigs following the secondary immunization (Day 109 - 130) (Chapter 8). 
To conclude, this thesis confirms that environmental enrichment has positive effects on the behaviour of pigs. It also indicates that switching from enriched to barren conditions is more detrimental for pigs' behaviour than housing in barren pens throughout. Effects of environmental enrichment on behaviour in tests of affective state were equivocal. Enriched housed pigs appeared less fearful in a novelty test in their home pen, but showed more signs of fear in an individual novel environment - novel object test and an attention bias test. Pigs from barren early life housing conditions seemed to be more sensitive to reward loss, irrespective of their current housing. Both early life and current housing, and a switch in housing conditions influenced specific antibody levels and the frequencies of leukocyte subpopulations in pigs, and the immune system seems to be alerted by changes in the environment. The results of this thesis underline the importance of enrichment for pigs, as well as a fit between their early and later life housing conditions. 


\section{Acknowledgements}

I arrived in the Netherlands on $10^{\text {th }}$ September2015 and started my PhD and new life in this peaceful and lovely city --- Wageningen. Beautiful time always flies and now it is the time to look back and express my gratitude to all those people whom I met and who helped me throughout my PhD study.

First, I would like to express the deepest appreciation to my promotor Prof. Bas Kemp, who gave me the chance to join ADP and do my PhD here. In September 2014, I first emailed him and asked whether there was a possibility for me to do a $\mathrm{PhD}$ in this group. I was so happy to receive his quick reply and was very lucky to have the chance to be interviewed. To me, he is the person who opened the door of ADP for me and then guided me in the new world of PhD.

Besides my promotor, I would like to give my sincere gratitude to my daily supervisors J. Elizabeth (Liesbeth) Bolhuis and Henk Parmentier for the continuous support and guidance of my PhD study and life, for their patience and immense knowledge. They are two different persons with totally different supervising styles. I feel like to be involved in a family with Mom Liesbeth and Dad Henk. Liesbeth is a detail-oriented 'workaholic', and it is same with most mothers in this world --- they all care about their little ones and be responsible. She always replied my emails quickly and gave construct suggestions. I did not have any background about animal behavior's and data analysis with SAS, so she taught me from zero. I would say that all my papers could not have been published without her. Henk is a special person who is a "hefty man" with a soft heart. He likes to make jokes and make people feel relaxed. When I had questions about immunology or I questioned myself, he 
always helped me and encouraged me. He is the one who told me that it is correct to keep yourself safe when you are working with animals, when I blamed myself to leave caretakers there with "crazy" sows.

My dear promotor and supervisors convincingly guided and encouraged me to be a professional and independent researcher in animal sciences. Without their persistent help, I cannot finish my PhD project successfully. It is wholeheartedly appreciated that your great supervision for my PhD, and I wish all of you to be healthy and happy forever.

Except my daily supervisors, there is another person who also helped me a lot in my project. That is dr. Inonge Reimert. She is the big sister in this family, as she was also supervised by Liesbeth during her $\mathrm{PhD}$, and she knew "Liesbeth style". She contributed a lot in my project, and she told me what Liesbeth would do in some situations, especially when Liesbeth was ill. She also helped me with my animal experiment, data analysis and paper writing. Whenever I went to her office and asked for help, she immediately started to answer my questions and search all information I needed.

Here, I would like to give my great thanks to China Scholarship Council (CSC) for their financial support for my living in the Netherlands.

Many thanks to the secretaries of ADP, Nanette van Hapert and Lora vander Kleijn for helping with administrative matters. Nanette, thanks for helping me to start the new life in this city and to reach the GP and dentist when I need them. Lora, thanks for sending me home when I was sick and sharing your experience to be a mom when I was pregnant. All my friends from other groups envy of our super nice secretaries, as you are always helpful. 
I wish to express my great gratitude to all the people who helped with my experiments. Manon van Marwijk, thanks for helping me with my animal experiment. The work with pigs was mostly tiring and sometimes was dangerous, but you were always there with me. Monique Ooms, thanks for helping with experiment design and arrangement. I was shocked by the excel file of my experimental plan made by you, which was very detailed and informative. Ger, thanks for helping me with ELISA and teaching me how to behave in the laboratory. When I made mistakes, you were so kind to tell me that everyone could make mistakes in the lab work and helped me to repeat the experiment. Joop Arts, who also helped me with ELISA, thanks for all the talks with you, and the knowledge about immunology and lab work you shared with me. Rudie Koopmanschap, thanks for helping with the saliva analysis, and all the jokes you made during the breaks. Oh, also thanks for driving me to the dentist! Bjorge Laurenssen, thanks for helping with my animal experiment and especially for the blood sampling when you were ill. Lisette Graat, thanks for your statistical support for the successive negative contrast test paper. Elske N. de Haas, thanks for your help in the animal tests.

Of course, without the help from the animal caretakers in Carus, I cannot finish my work. Rinie Ernste and Arjan van Dolderen, thanks for all your help in my animal experiment and I need to apologize that I escaped from the aggressive sow when you were "fighting" with her. Thanks are also giving to the caretakers who helped with the pen and experimental room construction, and cleaning of the rooms.

Special thanks to Christine Jansen and the technicians who helped me with lab work at Utrecht University. Christine, thanks for your help with FACS 
analysis and comments to improving the paper.

I would like to recognize the invaluable assistance from the students involved in my project. Nienke Kerssen, Sharine Smeets, Jettie Kolkman, and Qiying Yang, many thanks for all your work in my project and I am lucky to know all of you.

I would like to thank all my former and current colleagues in ADP, thanks for providing a friendly and open environment, and also thanks for all the supports from you throughout these years. Juncai Chen (陈俊材), thanks for picking up me in the airport with your wife Tingting, when I first arrived in the Netherlands. You helped me to start the new life in Wageningen and introduced your friends to me. I will remember all the talks and drinks with you and wish you and Tingting a happy life. Sofie van Nieuwamerongen - de Koning, you are the first person who brought me to Carus and showed me how to do behaviour observations with pigs. That was also the first time I got to know how big the boars were. Thanks for inviting me to be your paranymph and the witness of your wedding with Danny. Danny de Koning van Nieuwamerongen, thanks for inviting me to PhD dinners and drinks when you were in ADP, and keeping organizing Friday lunches until now. Pauline Krzysica, you are always funny and make me laugh. Novi Mayasari, you are always kind, warm, and funny. Francesca Marcato, my dear, thanks for all your smiles and hugs. Even we were very busy in these two years, you still came to hug me when you had a small break. Junnan Ma (马俊南), I enjoyed all the time we stayed together and hanged out, and thanks to be one of the witnesses of my marriage and pregnancy. Francesca and Junnan, also thanks for being my paranymphs, even I cannot defense in Aula due to Corona. I 
think we will have very nice party later in this year to celebrate my promotion to be a doctor and a mom. Anouschka Middelkoop, thanks for helping me with the animal experiment and always sending me the information which I needed. Bas Rodenburg and Jerine van der Eijk, thanks for your company when we went to Canada for the ISAE conference. Aart Lammers and Ariette van Knegsel, thanks for all the talks with you and your encouragements, and Aart thanks for the suggestions for my immunological experiments and papers. Kristina Simon, thanks for your warm welcome when I was new in ADP, and thanks for the handmade Germany cookies and bread. I also want to thank Ampai Nangsuay, Tom Berghof, Wei Xu, Carol Siyza da Sukva, Michel Verwoolde, Michel Verwoolde, Bahadir Guz, Eline Burgers, and Lisette van der Zande, as I appreciated to work in the same office with you.

Life of the PhD is not only about work, but also includes wonderful personal time with friends, therefore, I want to give my thanks to my friends in the Netherlands. Hui (田卉) is the first friend I made in Wageningen, and we shared a lot of time in the first year in our PhD. I will never forget your kindness and friendship. Without your company, I could not have gotten used to the new life that quickly. Xu Cheng (程旭), my big brother (我们全家 的人生导师), thanks for your care and guidance in my study and personal life. You are the uncle of my daughter and one of our family members forever. Bee (林正仪), thanks for all the delicious food from you and you are always our “大 Bee 姐”. Jialun (吴加伦), thanks for your 'cuteness' and nice temper, so that we can always make jokes with you. Xi Bai (白溪) and Peicheng Sun (孙培成), thanks for your friendship and I was touched by the food cooked by you after I delivered my baby girl. Liu Liu (刘汶), dear little sister, thanks 
for all the time with you and being one of my bridesmaids during my wedding. Hope we can meet each other soon and more. My thanks are also going to Jenny Soellner, Han Yan (严寒), Wenbo Wu (伍文博), Liuchang Nie (聂汶畅), Yuzhi Chen (陈禹志), Shuqing Yang (杨叔青), Caifang Wen (文彩芳), Shan Zhao (赵珊), Xiao Dong (董笑), Qiqi Lu (鲁琦琦), Qi Zheng (郑琪), Lei Mao (毛磊), Wenbiao Shi (石文标), Lu Chen(陈琒), Yuan He(何源), Zhongnan Chen (陈中南), Yuan He (贺远), and Yue Zhao (赵越) for all the friendship from you and all the time spent with you.

I would like to express my thanks to the friends from Lab of molecular biology: Martinus, Lucas, Carolien, Yuda, Jieyu Liu (刘洁宇), Tian Zeng (曾添), Jing Wang (王景 and your wife Lili Wei 魏丽莉), Fang Tang (唐芳), Huchen Li (李 虎臣), Defeng Shen (申德峰), Yueyang Ge (葛月阳，谢谢你和俊迪做我们 的伴娘和伴郎, 也谢谢你们给我带续命的稻香村), Jundi Yan (间俊迪), Siqi Yan (严思奇), Fengjiao Bu (卜凤娇), Haolin Zhang (张昊琳), Qian Li (李 茜), Tingting Xiao (肖婷婷), Peng Wang (王鹏), Mengmeng Hou (侯蒙蒙), Jianyong An (安剑勇), I am glad to know all of you and thanks for all the lunches, dinners, and parties with you.

I further thank my best friends in China: Jin Li (李进), Yang Li (李阳), Junhao Zhang (张峻豪), Jianyu Wu (伍健瑜), Zeli Xu (徐泽利), and Sen Feng (冯森). We have been friends for 10 years, and the time with you guys are full of happiness. I always think that we are not only friends, but also families.

Special thanks are going to Min Zhu (朱敏), the wife of Sen Feng, for helping with the cover design of my thesis. 
Here I also want to give my gratitude to my Dutch teacher Marie-José Leenaerts. Bedankt voor uw geduld in de cursus, en $u$ bent zo aardig en vriendelijk.

My dear baby girl, Zhizhi Yan (严知之), thanks for choosing me to be your mom. You were in my belly during the whole period of the thesis writing, so you sort of grew with this thesis. I am so proud of myself that I had you during my PhD, and I hope you are also proud of me. I have too many wishes for you, the same as other parents in this world, and I wish I can give all the best to you. I know, you will grow up, and you will have sadness and difficult times, so I hope you can be independent, brave, and optimistic. Never forget you have a mom and dad who love and support you in your whole life.

My beloved husband, Zhichun Yan (严志纯)! We were living in two different provinces in China, with $1200 \mathrm{~km}$ of distance, so the possibility to know each other was almost zero. It could be destiny that we met each other in the Netherlands, which is $7900 \mathrm{~km}$ far away from China. Here, I want to thank all your support in my work and life, and thanks for sharing your life with me! No word can express my love for you, 愿得一人心, 白首不相离!

最后，我要感谢我的妈妈，谢谢您的养育和对我的各种支持，谢谢您 给我完整的爱，谢谢您的无私奉献，谢谢您不远千里来荷兰帮我照顾 知之。我也要谢谢我的另一对父母, 谢谢您们对我和志纯的爱, 谢谢 您们让我成为您们的家人。为人父母方知父母恩，初为人母，我更明 白了父母将我们养育成人有多少艰辛和对我们的爱有多伟大! 愿您们 都健康平安, 以后的日子让我们来守护您们。 
Lu Luo

罗露

$24^{\text {th }}$ April 2020 


\section{Curriculum Vitae}

Lu Luo was born on $18^{\text {th }} 1991$ in

Sichuan Province, China. In 2013, she obtained her bachelor's degree at Department of Animal Medicine in Sichuan Agricultural University, Sichuan, China. After receiving her bachelor's degree, she continued her master study in Sichuan Agricultural University, focusing on preventive veterinary medicine. Her MSc thesis was about the diagnose of Lawsonia

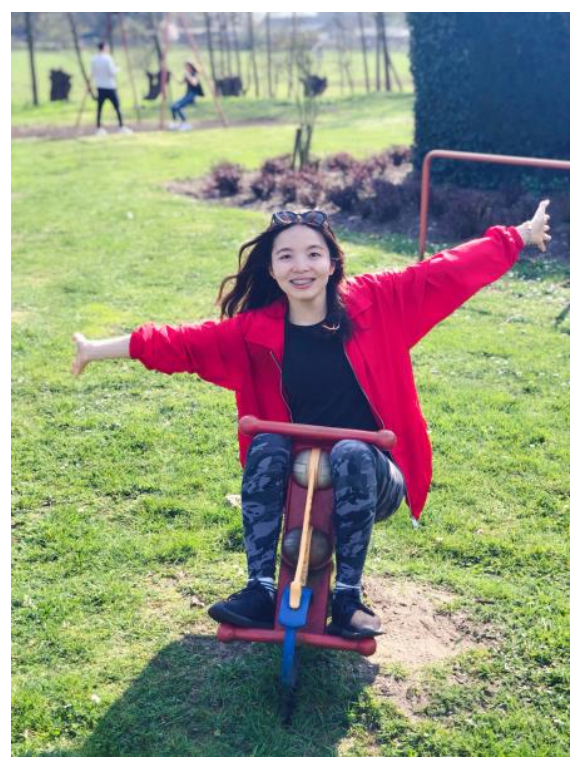
intracellularis in pigs by Loop-mediated Isothermal Amplification and PCR, and she graduated in 2015 with a MSc degree. Except pigs, she also had experience of working on giant pandas and some wild animals during her master, and she developed more interests in animal science, and started to search for PhD projects. In September 2015, she started her PhD in Adaptation Physiology Group, Wageningen University \& Research. Her PhD project aimed to investigate the effects of early life and current environmental enrichment on behaviour, affective state and immunity in pigs, and the results of this project are presented in this thesis. 


\section{Publications}

Luo, L., Jansen, C.A., Bolhuis, J.E., Arts, J.A.J., Kemp, B., Parmentier, H.K. (2020). Early and later life environmental enrichment affect specific antibody responses and blood leukocyte subpopulations in pigs. Physiology \& Behavior, 217: 112799

Luo, L., Reimert, I., de Haas, E.N., Kemp, B., \& Bolhuis, J.E. (2019). Effects of early and later life environmental enrichment and personality on attention bias in pigs (Sus scrofa domesticus). Animal Cognition, 22.6: 959-972

Luo, L., Reimert, I., Graat, E.A.M., Smeets, S., Kemp, B., Bolhuis, J.E. (2019). Effects of early life and current housing on sensitivity to reward loss in a successive negative contrast test in pigs. Animal Cognition, 23(1), 121-130

Luo, L., Geers, R., Reimert, I., Kemp, Bas., Parmentier, H.K., \& Bolhuis, J.E. (2017). Effects of environmental enrichment and regrouping on natural autoantibodies-binding danger and neural antigens in healthy pigs with different individual characteristics. animal, 11 (11): 2019-2026

Luo, L., Van Dixhoorn, I.D.E., Reimert, I., Kemp, B., Bolhuis, J.E., \& Parmentier, H.K. (2017). Effect of enriched housing on levels of natural (auto-)antibodies in pigs co-infected with porcine reproductive respiratory syndrome virus (PRRSV) and Actinobacillus pleuropneumoniae. Veterinary Research, 48 (1): 75 
Wageningen Institute of Animal Sciences

Education Statement

Issued to: Lu Luo

The Graduate School

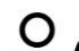

Date: 24 April 2020

Group: Adaptation Physiology Group (ADP)

\begin{tabular}{|l|l|}
\hline A. The Basic Package & year \\
WIAS Introduction Day (mandatory) & 2015 \\
Course on essential skills (Frank Little) (recommended) & 2015 \\
Ethics and Philosophy in Life Sciences (mandatory) & 2016 \\
\hline \multicolumn{2}{|c}{ Subtotal Basic Package } \\
\hline
\end{tabular}

\section{B. Disciplinary Competences}

year

Discussion Group WIAS-Animal Immunology and Health 2015

Preparing own PhD research proposal 2015

Laboratory Course on Animal experiments 2016

Statistic for the Life Sciences 2016

Design of Experiments 2016

Species specific course: pig 2017

Genetic methods for improving adaptation to group housing systems

The fundamentals of animal emotion

\section{Professional Competences}

Techniques for writing and presenting a scientific paper

Project and Time Management

Data management planning 2016

WGS PhD workshop Carousel

Communication with the media and the general public 
Effective behaviour in your professional surroundings

2018

Supervising BSc and MSc Thesis students

Reviewing a scientific paper

Scientific publishing 2018

The final touch: writing the general introduction and discussion 2019

High-impact Writing in Science

D. Presentation Skills (maximum 4 credits)

Talk: 'Effect of enriched housing on levels of natural (auto)year antibodies in pigs co-infected with Porcine reproductive and respiratory virus (PRRSV) and Actinobacillus pleuropneumoniae (A. pleuropneumoniae)' - 4th Wageningen PhD Symposium, the Netherlands

Poster: 'Early and later life environmental enrichment affect leukocyte subpopulations in pigs' - 6th European Veterinary Immunology Workshop, the Netherlands

Talk: 'Effects of early and later life environmental enrichment on the response to reward loss in pigs in a successive negative contrast test' - Conference of the International Society for Applied Ethology (ISAE), Prince Edward Island, Canada

Talk: 'Effects of early and later life environmental enrichment and coping style on attention bias in pigs' - ISAE, Bergen, Norway

* A credit represents a normative study load of 28 hours of study 

The research described in this thesis was financially supported by the Adaptation Physiology Group, Department of Animal Science, Wageningen University.

Lu Luo was sponsored by the China Scholarship Council.

Layout design by: Lu Luo \& Zhichun Yan

Cover design by: Min Zhu

Printed by: $\quad$ ProefschriftMaken 